University of Louisville

ThinkIR: The University of Louisville's Institutional Repository

Electronic Theses and Dissertations

$12-2004$

\title{
Human visual processing of orientation in broad stimuli.
}

Bruce C. Hansen

University of Louisville

Follow this and additional works at: https://ir.library.louisville.edu/etd

\section{Recommended Citation}

Hansen, Bruce C., "Human visual processing of orientation in broad stimuli." (2004). Electronic Theses and Dissertations. Paper 572.

https://doi.org/10.18297/etd/572

This Doctoral Dissertation is brought to you for free and open access by ThinkIR: The University of Louisville's Institutional Repository. It has been accepted for inclusion in Electronic Theses and Dissertations by an authorized administrator of ThinkIR: The University of Louisville's Institutional Repository. This title appears here courtesy of the author, who has retained all other copyrights. For more information, please contact thinkir@louisville.edu. 
HUMAN VISUAL PROCESSING OF ORIENTATION IN BROADBAND STIMULI

By

Bruce C. Hansen

B.S., University of Michigan-Flint, 2000

M.A., University of Louisville, 2003

\author{
A Dissertation \\ Submitted to the Faculty of the \\ Graduate School of the University of Louisville \\ in Partial Fulfillment of the Requirements \\ for the Degree of
}

Doctor of Philosophy

Department of Psychological and Brain Sciences

University of Louisville

Louisville, Kentucky

December 2004 
HUMAN VISUAL PROCESSING OF ORIENTATION IN BROADBAND STIMULI

By

Bruce C. Hansen

B.S., University of Michigan-Flint, 2000

M.A., University of Louisville, 2003

A Dissertation Approved on

November 5, 2004

by the following Dissertation Committee:

Dissertation Director 


\section{DEDICATION}

This dissertation is dedicated to my parents:

Mr. James Carl Hansen

and

Mrs. Renee Jacqueline Hansen

for providing me with endless opportunities by instilling in me the strength, determination, perseverance, and self-discipline in order to achieve any goal. 


\section{ACKNOWLEDGEMENTS}

I would like to thank Dr. Edward A. Essock for his careful guidance, considerate patience, and endless support as well as for providing me with the opportunity to engage in graduate study. I would also like to thank my dissertation committee members: Dr. Paul J. DeMarco, Dr. George D. Mower, Dr. Heywood M. Petry, and Dr. Fred Wightman for their thoughtful comments regarding this dissertation. My sincerest gratitude is also extended to Dr. Thomas A. Wrobel and Dr. Paul M. Bronstein for their support and for providing me with a strong foundation in research methods. My gratitude is also extended to Dr. J. Kevin DeFord and Dr. Michael J. Sinai for their guidance during my first year of graduate study. I would also like to thank Dr. Yufeng Zheng, Dr. Pinakin Gunvant, and Andrew M. Haun for their valuable and challenging comments regarding this dissertation. During my tenure as a graduate student, I was privileged to have such colleagues as Thomas E. Dawson, J. Isaiah Harbison, Nathan Johnson, Scott LaJoie, James Massey, Julia Robinson, Liz Snyder, and Bing Wu with which I have enjoyed many interesting discussions and shared many laughs. Lastly, I extend sincere gratitude to my immediate family for always believing in me, and to my longtime friend Shannon Estep for her continued support. 


\title{
ABSTRACT \\ HUMAN VISUAL PROCESSING OF ORIENTATION IN BROADBAND STIMULI
}

\author{
Bruce C. Hansen
}

November 5, 2004

Recently our lab has shown that with broadband stimuli (either visual noise or natural scenes), performance for detecting oriented content is worst at horizontal, best at the obliques, and intermediate at vertical orientations -- an anisotropy (termed the "horizontal effect") quite different from the well-known "oblique effect" (worst performance at obliques) obtained with simple line or grating stimuli. This horizontal effect can be explained by a proposed anisotropic normalization model that operates at the level of striate cortex by implementing the known numerical biases of striate neurons preferring different orientations as well as the strength of those responses from neurons tuned to similar orientations and spatial frequencies (with that strength being dependent on the spatial relationships between different scales and orientations present in the stimuli). To assess how the proposed striate normalization mechanism might operate when the visual system is presented with broadband stimuli containing different amounts of spatial frequency and orientation content, two suprathreshold matching experiments were conducted. Additionally, to provide an estimate of how broadband stimuli might modulate the weights of the proposed model, a series of neural response simulations were carried out on different types of broadband natural scene imagery. The stimuli for the 
psychophysical experiments were generated by making broadband isotropic visual noise patterns and filtering their amplitude spectra to contain a test increment across a specified range of orientations and spatial frequencies. The extent of the test increment's orientation and frequency bandwidth was systematically varied. A standard psychophysical matching paradigm was used to assess the perceived strength of the oriented structure in a test pattern relative to the oriented structure in a comparison pattern. The results yielded the traditional oblique effect when a fairly small range of orientations and high spatial frequencies were incremented and the horizontal effect was observed for broadband increments of about $20^{\circ}$ and 1-octave in frequency and larger. A blend of the two anisotropies was observed at intermediate increment bandwidth. The results of the psychophysical experiments were discussed in the context of the proposed striate normalization model with added insight from the results of the neural response simulations. 


\section{TABLE OF CONTENTS}

PAGE

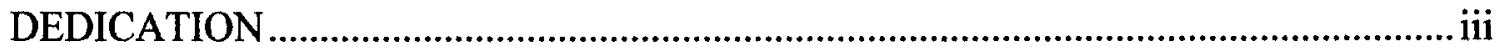

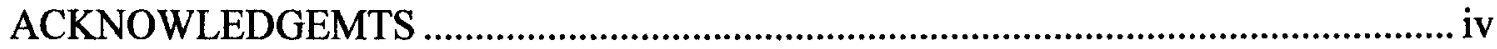

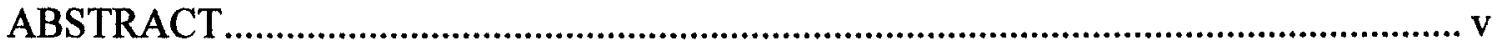

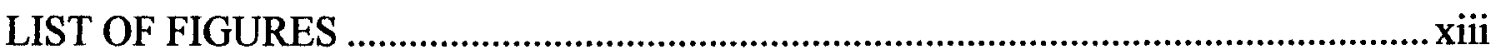

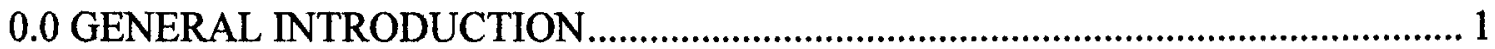

1.0 THE STRUUCTURAL REGULARITIES

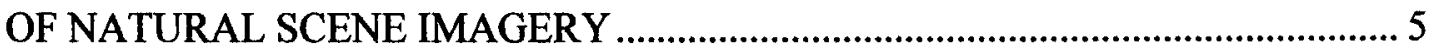

1.1 Content Regularities with Respect

to Different Spatial Scales ................................................................................... 6

1.1.1 Characteristic Slopes for Different

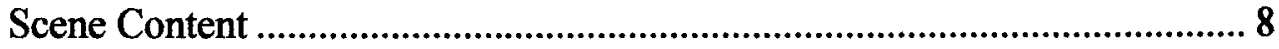

1.2 Content Regularities with Respect

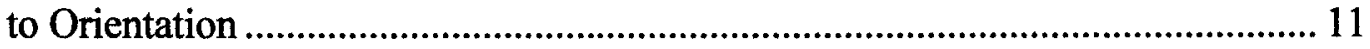

2.0 HUMAN PERCEPTUAL PERFORMANCE AND THE

CONTENT REGULARITIES OF NATURAL SCENES ......................................... 19

2.1 Human Perceptual Performance and

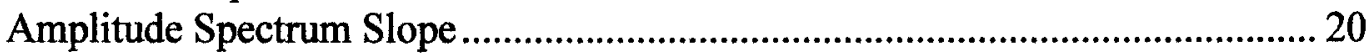

2.1.1 Broad-scale Visual Adaptation of

Natural Scene Imagery ................................................................................. 20

2.1.2 Detection and Discrimination of Changes

in the Spectral Slope of Natural Scenes.................................................... 22

2.1.3 The role of Spectral Slope in Discriminating

Between Different Scene Content.................................................................. 25

2.2 Human Perception of Orientation in

Broad-scale Imagery. 
2.2.0 Human Perception of Orientation:

The Oblique Effect.

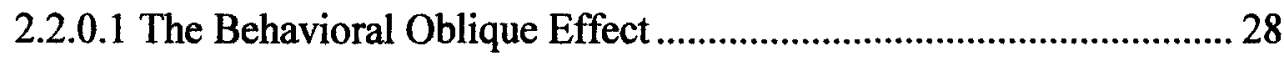

2.2.0.2 The Physiological Oblique Effect..................................................29

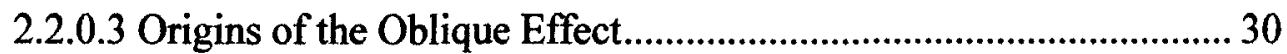

2.2.1 Human Visual Perception of Oriented

Content in Broadband Noise

2.2.2 Human Visual Perception of Oriented

Content in Natural Scene Stimuli

2.2.2.1 Visual Processing of Orientation in Natural Scene Stimuli: Part I

2.2.2.2 Visual Processing of Orientation in Natural Scene Stimuli: Part II

2.2.2.3 Visual Processing of Orientation in Natural Scene Stimuli: Part III

\subsection{TOWARD A STRIATE PROCESSING MODEL OF} BROADBAND NATURAL SCENE CONTENT.

3.1 The Inherent Orientation Processing Anisotropy:

A Horizontal Effect

3.2 Dynamic Orientation Processing Anisotropies:

The Content-Dependent Effects

4.0 A NEW MODEL OF NEURAL PROCESSING

IN STRIATE CORTEX

5.0 GENERAL METHODS.

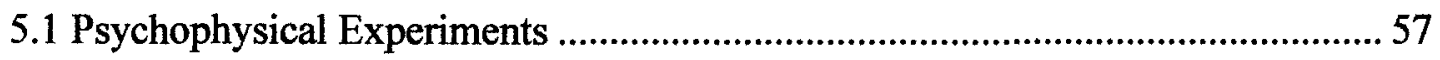

5.1.1 Experiment 1

5.1.1.1 Stimulus Generation for

Experiment 1 
5.1.1.2 Psychophysical Paradigm for

Experiment 1

5.1.2 Experiment 2

5.1.2.1 Stimulus Generation for

Experiment 2.

5.1.2.2 Psychophysical Paradigm for

Experiment 2 .

6.0 PSYCHOPHYSICAL EXPERIMENT RESULTS

6.1 Experiment 1 Results .72

6.1.1 Planned Comparisons: The Interaction Across All Conditions.

6.1.2 Planned Comparisons: Interactions Within Spatial Frequency/Orientation Bands

6.1.2.1 Planned Comparisons: Interactions

Within Spatial Frequency Bands

6.1.2.2 Planned Comparisons: Interactions

Within Orientation Bands

6.1.3 Planned Comparisons: Interactions

Within Each Condition

6.1.4 General Summary of the Results from Experiment 1

6.2 Experiment 2 Results 77

6.2.1 Planned Comparisons: The Interaction Across All Conditions.

6.2.2 Planned Comparisons: Interactions Within

Spatial Frequency/Orientation Bands 78

6.2.2.1 Planned Comparisons: Interactions

Within Spatial Frequency Bands 78

6.2.2.2 Planned Comparisons: Interactions

Within Orientation Bands 79 
6.2.3 Planned Comparisons: Interactions

Within Each Condition

6.2.4 General Summary of the Results

from Experiment 1

6.3 The Interactions Between Experiment 1

and Experiment 2

6.3.1 Planned Comparisons: The Overall Interaction

Between Experiments 1 and 2

6.3.2 Planned Comparisons: The Individual Interactions

Between the Two Experiments

6.3.3 General Summary of the Interactions Between

Experiments 1 and 2

\subsection{PROTOCOL FOR MODELING THE THREE DIFFERENT}

CONTENT-DEPENDENT EFFECTS

7.1 Verifying the Content-Dependent

Weighting System.

7.2 The Neural Response Simulation Protocol ........................................................... 88

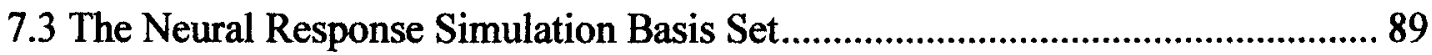

7.4 Simulated Neural Response Procedure …….......................................................... 91

7.5 Image Sets Used in Constructing the

Content-Dependent Effects Weights

7.5.1 Content-Dependent Effect 1:

Fixed Content-Bias Weights.

7.5.2 Content-Dependent Effect 2:

Variable $\alpha$ Weights .95

7.5.3 Content-Dependent Effect 3: Variable

Horizontal Content Bias Weights .96

7.6 Results: Content-Dependent Effects

Neural Response Simulations 
7.6.1 Neural Response Simulations for Content-Dependent Effect 1

7.6.2 Neural Response Simulations for

Content-Dependent Effect 2

7.6.3 Neural Response Simulations for

Content-Dependent Effect 3

7.6.4 Neural Response Simulation

General Results

8.0 GENERAL DISCUSSION

8.1 Implications from the Current

Psychophysical Experiments

8.1.1 Implications from the No-background

Experiment

8.1.2 Implications from the $1 / f$ Noise

Background Experiment

8.2 The Weighting Indices for the Proposed

Striate Normalization Model.

8.2.1 The Inherent Processing Bias

Weighting Indices

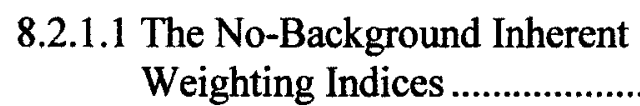

8.2.1.2 The Noise Background Inherent

Weighting Indices

8.2.2 The Interactions Between the Two

Inherent Weighting Indices.

8.3 The Content-Dependent Processing

Bias Weighting Indices

8.4 A Schematic Representation of the Proposed

Striate Normalization Model 


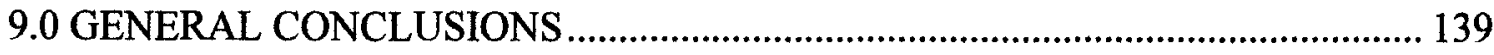

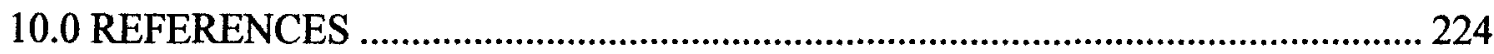

11.0 APPENDIX

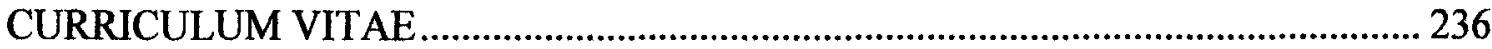




\section{LIST OF FIGURES}

FIGURE

PAGE

1. Example of the building up of a complex 2D signal ............................................... 153

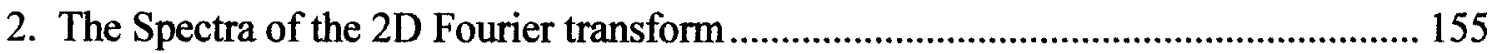

3. Characterization of natural scene amplitude spectrum slope.................................... 156

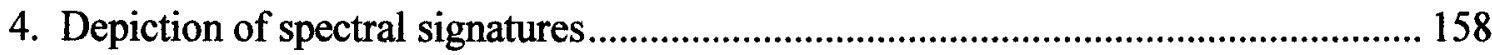

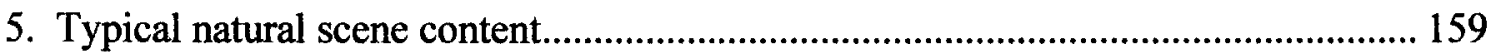

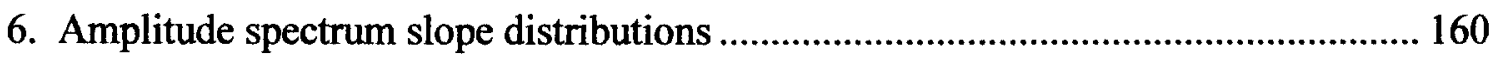

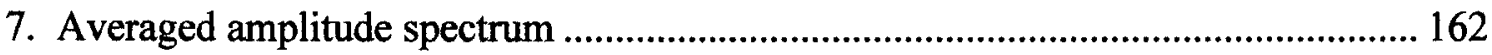

8. Schematic depicting the calculation of orientation biases ....................................... 164

9. Schematic depicting the parsing of a given amplitude spectrum............................ 165

10. Orientation analysis of natural scene content ...................................................... 166

11. Average amplitude bias for 30 sampled orientations............................................. 167

12. Amplitude biases for 20 spatial frequencies for 60 sampled orientations .............. 168

13. Noise patterns with different amplitude spectrum slopes ....................................... 169

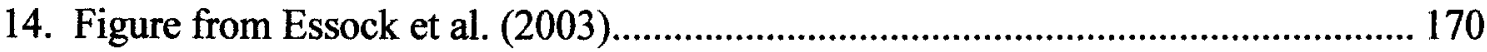

15. Psychophysical data showing the horizontal effect ............................................ 172

16. Natural scene images with differently oriented content biases............................... 174

17. Illustration of the application of the triangle increment........................................... 175

18. Psychophysical data showing the content-dependent effect 1 ............................... 177

19. Natural scene with different amplitude spectrum slopes ........................................ 178 
20. Psychophysical data showing the content-dependent effect 2

21. Natural scene images with variable differently oriented content biases.

22. Psychophysical data showing the content-dependent effect 3

23. Example 2D joint histograms.

24. Averaged amplitude spectrum from horizontal content biased scenes.

25. Illustration of some of the methodology for Experiment 1.

26. Sixteen-cell matrix with 2D examples of the different increment extents

27. Illustration of the suprathreshold matching procedure

28. Illustration of some of the methodology for Experiment 2 .

29. Psychophysical data from Experiment 1.

30. Psychophysical data from Experiment 2 .

31. Psychophysical data from Experiments 1 and 2

32. Spatial and Fourier domain representations of the basis filters

33. Illustration of the neural response simulation procedures

34. Illustration of the neural response simulation analysis procedures 200

35. Illustration procedures implemented in the joint histogram analysis 201

36. Typical averaged joint histograms for the neural simulations 202

37. Content-dependent effect 1 weighting index 204

38. Content-dependent effect 2 weighting index .206

39. Content-dependent effect 3 weighting index 208

40. Inherent weighting indices from Experiment 1 210

41. Inherent weighting indices from Experiment 2

42. Vector samples from the inherent weighting indices. 
43. Schematic of the normalization pool's extent.

44. Schematic representation of the inherent weighting index implementation............ 221

45. Full schematic representation of the proposed striate normalization model............ 223 


\section{GENERAL INTRODUCTION}

Within the past decade there has been a concerted effort to relate the spatial characteristics of the content contained in real-world scenes to the processing strategies of the visual system. Specifically, it has been posited that the biological processes that underlie visual encoding are tuned to optimize the encoding of the spatial relationships present in the content of the visual world (e.g., the spatial scale, orientation, luminance and color contrast, and texture characteristics that form the structural content of the visual image). Accordingly, these spatial relationships of the content contained in real-world imagery ${ }^{1}$ have been extensively examined and quantified by numerous techniques, some of which include optical and digital Fourier analysis, higher-order autocorrelation analyses, principal component analysis, and convolution with various types of gradient kernels (Switkes, Mayer, \& Sloan, 1978; Burton \& Moorhead, 1987; Tolhurst, Tadmor, \& Chao, 1992; Van der Schaaf \& van Hateren, 1996; Keil \& Cristóbal, 2000; Oliva \& Torralba, 2001; Thomson, 2001a; Baddeley \& Hancock, 1991; Craven, 1993; Coppola, Purves, McCoy, \& Purves, 1998; Howe \& Purves, 2002; Hansen \& Essock, 2004a; 2004b). Regardless of the analysis technique utilized, or the scene specific content (e.g., trees/grasslands or buildings/roadways) in the types natural scene imagery investigated, two general regularities of spatial content are found: (1) The amount of physical energy (or intensity) of any real-world image peaks at the larger spatial scales (i.e., lower spatial

\footnotetext{
${ }^{1}$ Specifically, any stimulus possessing semantically meaningful spatial content (whether or not it contains carpentered/manufactured content) at a broad range of spatial scales will be referred to here as a 'natural' or 'real-world' scene.
} 
frequencies) and decreases with increasing spatial scale (i.e., higher spatial frequencies) of the image content, and (2) for typical scenes, an anisotropy with a bias in image content favoring the horizontal and vertical axes (cardinal axes) relative to the oblique axes ( $45^{\circ}$ and $135^{\circ}$ oblique). Sections 1.1 and 1.2 will examine these regularities of spatial scale and orientation while emphasizing results obtained with the most frequently used method of measurement, that being Fourier analysis.

Given that the above mentioned regularities are generally encountered, regardless of the type of environment in which they were measured, several researchers have proposed that it would be to the benefit of the visual system to take advantage of such regularities in encoding image information. However, such statements have left vision researchers with the question of identifying how, if at all, the visual system could make use of such regularities. Since these regularities are often global in nature (that is, typically distributed throughout an entire image), one effective means for investigating the extent to which visual processing depends on such regularities is through visual performancebased behavioral experiments. Specifically, one could vary the amount of content present across spatial scales, or at specific orientations, or both, in order to determine (via performance) which conditions are ideally suited for the effective processing of visual information. That is, if visual perceptual-performance depends on the presence of these regularities, any deviation from those regularities will result in less than ideal performance. As it will be discussed, such an evaluation is not as clear cut as it seems, and is often dependent on the experimental paradigm utilized. 
The vast majority of studies that have concentrated on examining whether or not the visual system is optimally suited to process visual scenes that possess the previously mentioned regularities of image content, are still very much in the early phases of investigation. Much of the work that has been done, to date, includes studies that measure the regularities of image content and then emphasize parallels between those regularities and certain traditionally evaluated visual performance phenomena. Others have taken this a step further by empirically measuring the extent to which experimental manipulation of the regularities of natural stimuli can impact visually driven performance. It is the latter of the two that will be the focus of review in Sections 2 and 3. To date, most empirical investigations have focused on whether or not the typical distribution of image content across the multiple spatial scales contained in natural scenes yields maximal performance when compared to performance with natural scene stimuli containing distributions of image content that deviate from the typical distribution. That is, visual performance has typically been evaluated as a function of changes in the distribution of image content across multiple spatial scales, regardless of any differences in content distributions at the different orientations. Only recently has there been a drive to investigate visual processing of natural scene stimuli as the amount of content at different orientations is varied which will be reviewed in Section 3.

The subsequent sections will thus present the current state of research with respect to understanding the general spatial relationships of the content contained in typical realworld imagery and visually driven performance-based experiments as they relate to those structural regularities most often reported in the literature. Specifically, it will be shown 
that while some parallels have been drawn between the observed content regularities of natural scenes and the occurrence of certain visual phenomena, such parallels are not supported when directly assessed. Before moving into a discussion of the current status of the area of research devoted to measuring visual performance with respect to natural scene content regularities (Sections 2 and 3), the following section will first describe how these regularities are typically measured and subsequently reported. 


\section{THE STRUCTURAL REGULARITIES OF NATURAL SCENE IMAGERY}

The characteristics of any visual scene that human beings are typically confronted with is filled with an extensive amount of information that must be properly encoded, thereby enabling us to accurately perceive and interact with our surroundings. It goes without saying that the processes involved in this visual encoding are very dynamic and complicated, a fact to which any vision scientist can attest. However, throughout this century, especially the later half, a great deal about how the visual system functions has been uncovered. Of course with every new finding, there arose even more questions, some of which seemed almost impossible to answer. One reason for this comes from the fact that traditional studies on the human visual system often employed visual stimuli that consisted of checkerboard patterns, sine- and square-wave gratings, lines and edges, and random dot patterns. While the use of such stimuli offered much in terms of stimulus control, they are in no way representative of the visual environment that we have interacted with through our evolutionary development, or even the environment we are faced with throughout our early developmental stages. Such stimuli were primarily utilized as abstract components that make up our visual environments; these components were derived to stimulate various classes of visual neurons, independent of other visual neuron classes. While much has been learned about how different classes of visual neurons function in and of themselves, the final visual image that we perceive is a result 
of a complex interaction among these classes; a process that often involves lateral inhibition at a number of processing levels, sensitivity adjustments (i.e., adaptation processes), cortical feedforward and feedback processing loops that span across multiple processing areas, and the potential influence of higher-order attention mechanisms on these areas to name a few. The later considerations are compounded by the fact that the visual environment we encounter in our day to day lives changes dramatically with a simple turn of the head. However, recent discoveries by scientists from various disciplines have proposed that, regardless the type of visual scene projected onto the retina, there exist striking similarities in the structural content of such scenery.

\subsection{Content Regularities With Respect To Different Spatial Scales}

It may seem unlikely that, for example, an image of the Grand Canyon would have anything structurally in common with an image of one's own backyard, but there is a great deal of evidence that argues they are quite similar. One particular measurement showing such similarity is the global 2D Discrete Fourier Transform (DFT). Specifically, any complex waveform (in this case, an image is treated as a $2 \mathrm{D}$ complex luminance waveform) can be represented as the sum of sinusoidal waveforms of different amplitudes, frequencies, orientations and phases (see Figure 1). The amplitude plotted as a function of spatial frequency and orientation is often referred to as the amplitude spectrum and the phase of the waveforms as a function of orientation and spatial frequency is called the phase spectrum (Shapley $\&$ Lennie, 1985; Bracewell, 2000). The amplitude (or power/energy) spectra of different natural scene images have been measured in a number of studies (e.g. Field, 1987; Tolhurst, Tadmor, \& Chao, 1992; van 
der Schaaf \& van Hateren, 1996; Simoncelli \& Olshausen, 2001; Oliva \& Torralba, 2001), refer to Figure 2 for examples. A typical method of analysis involves examining global spectral properties as a function of spatial frequency. This involves averaging the amplitudes across orientation at each spatial frequency and plotting the results on logarithmic axes (refer to Figure 3a-c). The typical plot peaks at the lowest spatial frequencies and falls with increasing frequency, $f$, falling off by a factor of approximately $1 / f^{\alpha}$ in logarithmic coordinates (see Figure 3d). The exact exponent, $\alpha$, that characterizes natural scenes has been the subject of much debate, but the general consensus has been that of an observed range from $f^{0.6}$ to $f^{1.6}$ (Field \& Brady, 1997). In order to provide a more intuitive understanding of how a given image's amplitude spectrum fall-off (as described by $\alpha$ ) relates to the image contents, consider Figure 3e-g. The examples show three images containing the same type of content (outdoor grassland/trees), however, notice that the image that possesses numerous, globally distributed sharp edges has a relatively shallower $\alpha$ value compared to the other three examples. The preponderance of sharp edges translates to larger amplitudes in the higher spatial frequency range of its corresponding amplitude spectrum. Accordingly, the image possessing large luminance transitions with relatively small amounts of globally distributed sharp edges has an amplitude spectrum defined by a relatively steep $\alpha$. The large luminance transitions translate to larger amplitudes in the low spatial frequency range of its corresponding amplitude spectrum. 


\subsubsection{Characteristic Slopes for Different Scene Content}

The issue of determining the exact exponent, $\alpha$, that describes the fall-off of amplitude with increasing image scale is not a trivial one. As will be discussed in later sections of the current paper, the exponent of content-amplitude fall-off can determine the scale at which the visual system is most sensitive as well as determine the ability of humans to discriminate between different content in different natural scenes (Knill, Field, \& Kersten, 1990; Tadmor \& Tolhurst, 1994; Tolhurst \& Tadmor, 1997; Webster \& Miyahara, 1997; Párraga, Troscianko, \& Tolhurst, 2000; Párraga \& Tolhurst, 2000; Tolhurst \& Tadmor, 2000). In the literature devoted to measuring the distribution of content in large samples of real-world imagery (i.e., determine the typical $\alpha$ encountered in natural scenes), the image sets generally consisted of imagery ranging from purely naturalistic content (i.e., woodlands, meadows, general shrubbery, etc.) to images of purely carpentered structures (both indoor as well as outdoor structures) (Switkes, Mayer, \& Sloan, 1978; Burton \& Moorhead, 1987; Tolhurst, Tadmor, \& Chao, 1992; Van der Schaaf \& van Hateren, 1996; Keil \& Cristóbal, 2000). The $a$ distributions from those studies didn't reveal any obvious multi-modal tendencies, however, it could very well be the case that the primary reason behind such a large observed range of $\alpha$ values was due to the broad range of image content present in the imagery. The idea here is that, in a given type of environment (e.g., urban or rural), the range of $\alpha$ values encountered may be very small and when averaged together, any multimodal tendencies would likely be washed out. This is not a novel idea however, Oliva and Torralba (2001) and Torralba and Oliva (2003) have argued that the "spectral signature" (i.e., the nature in which power [squared amplitude] is distributed in a given spectrum across multiple spatial 
frequencies and orientations) of real-world imagery depends very much on the type of environment depicted in the imagery. While it wasn't the focus of their analyses, the $\alpha$ values they do report were obtained from within different orientation bandwidths, and were found to depend on scene category (i.e., "forest", "field", "coast", "street", "portrait" "high building", etc.) see Figure 4. Additionally, while the image sets were large, the size of the imagery was limited to $256 \times 256$ pixels and were gathered from variable sources, which leads to numerous unknown and potentially confounding influences on the analysis (e.g., images in the Corel stock photo library were sampled extensively; such images have likely been subjected to numerous image processing algorithms in order to enhance the aesthetic appeal of the imagery). An additional confound may have also been present in that, it isn't made entirely clear whether or not the imagery had been fully linearized (i.e., appropriately gamma corrected) before subjecting the imagery to content analysis. Nevertheless, $\alpha$ values were given (Torralba and Oliva, 2003) for cardinal and oblique orientations for imagery of purely naturalistic and purely carpentered environments. When these values are averaged across orientation within each category type, the difference is negligible: for naturalistic scenes, $\alpha=1.43$, and for carpentered environments, $\alpha=1.44$ ( $\alpha$ values have been corrected to reflect amplitude spectra fall-offs).

Given the limitations of the imagery discussed above, Hansen and Essock (2004b) sought to determine whether $\alpha$ values describing the orientation-averaged amplitude spectra of real-world imagery do in fact depend on the type of content present in the imagery. In their report, Hansen and Essock (2004b) compiled an image library of over 
1800 natural scene images ranging in content from purely naturalistic to images containing only indoor or outdoor carpentered materials (see Hansen, Essock, Zheng, \& DeFord, 2003; Hansen \& Essock, 2004a; 2004b for image acquisition and content analysis specifications and procedures). In order to conduct an analysis of imagery most likely to be encountered, Hansen and Essock (2004b) grouped imagery into three very general categories, specifically: purely naturalistic, mixed naturalistic and carpentered content, and purely carpentered content (see Figure 5a for examples). The image database contained 131 images (1024 x 1024 pixels) of carpentered environments, and thus served as a limiting factor in terms of the number of scenes selected for equal $n$ comparisons. One-hundred thirty-one images were randomly selected to comprise the other two images sets. As shown in Figure 6a, there was a clear multi-modal distribution of $\alpha$ values, with each peak corresponding to a different scene category and considerable overlap between all categories. Torralba and Oliva (2003) reported that the "scene-scale" (i.e., perceived distance of the primary structures that make up a given scene) of different imagery within their categories had an impact on the corresponding spectral signatures. This was also examined by Hansen and Essock (2004b), however, the number of carpentered and mixed naturalistic/carpentered images in their database was limited, thereby discouraging any meaningful analyses with respect to different scene-scales. That their naturalistic content image set exceeded 900 images, they were able to split this set into three sets (i.e., scene scale sets): close-up images, mid-range images, and farrange images (refer to Figure 5b for examples). This process involved placing obviously close-range imagery and far-range imagery into their respective categories; if there was any doubt with respect to how close or far the range of content in a given image was, it 
was automatically placed into the mid-range category. While the authors claim the selection process was carried out as objectively as possible, they admit that all possibility of experimenter bias in their selection process could not be ruled out. This categorization process resulted in 201 randomly sampled images from those sets in order to make equal $n$ comparisons between the different naturalistic scene-scale image sets. As shown in Figure $6 \mathbf{b}$, this categorization procedure yielded very similar $\alpha$ values, also the scenescale categorizations differ very little from the sample of naturalistic images depicted in

\section{Figure 5.}

The overall range of $\alpha$ values reported in Figure 6, $(\sim 0.59$ to $\sim 1.66)$ was very much in agreement with the typical range reported in the respective literature (i.e., $\sim 0.60$ to $\sim 1.6$ ). However, this range was greatly reduced when considering general environments one may encounter (e.g. Figure 5a). As mentioned earlier, the exponent of content-amplitude fall-off can determine the scale at which the visual system is most sensitive as well as determining the ability in which humans can discriminate between different objects in different natural scenes. This issue will be addressed further in Section 3 where the empirical studies that have measured visually driven performance using stimuli with variable $\alpha$ values will be reviewed.

\subsection{Content Regularities With Respect To Orientation}

The first type of regularity discussed in the preceding section involved analyses of the orientation-averaged amplitude spectra of real-world imagery. However, such an account ignores the variation of amplitude as a function of orientation. As mentioned in the 
introduction, there have been numerous studies that set out to measure the distribution of content in real-world imagery, with many of those investigations focusing on the distribution of content with respect to orientation. A number of methods have been utilized to measure orientation content-biases, including: Fourier analysis (Switkes, Mayer, \& Sloan, 1978; Van der Schaaf \& van Hateren, 1996; Keil \& Cristóbal, 2000; Oliva \& Torralba, 2001; Hansen \& Essock, 2004a; Torralba \& Oliva, 2003), second and higher-order autocorrelation analyses (Baddeley, 1997; Thomson, 1999; 2001a; 2001b), principle components analysis (Baddeley \& Hancock, 1991; Hancock, Baddeley, \& Smith, 1992), and a variety of convolution kernels (Craven, 1993; Coppola, Purves, McCoy, \& Purves, 1998). As mentioned earlier, typical natural scenes are found to be anisotropic with relatively less content at oblique orientations and the most content at orientations near horizontal and vertical (see Figure 7). The first study to address this, Switkes, Mayer, and Sloan (1978), used optical Fourier analysis and found that a prominant bias of power at the cardinal axes exists, and also observed the anisotropy regardless of whether the scene content was purely natural outdoor scenery or scenes of carpentered content. Studies of magnitude and direction of gradients in imagery (Coppola, Purves, McCoy, \& Purves, 1998) and of Fourier energy/amplitude examined as a function of orientation within weighted or sectored regions of images (Van der Schaaf \& van Hateren, 1996; Keil \& Cristóbal, 2000; Oliva \& Torralba, 2001; Hansen \& Essock, 2004a; Torralba \& Oliva, 2003) support the anisotropic distribution of image content.

An important question that arises out of the above mentioned oriented-content measurements is whether or not there is, on average, more horizontal content relative to 
vertical content. Unfortunately the answer has not been clear. The primary reason for this lies in the fact that of the studies that do report magnitudes for the different orientations, the image sample size was very limited, or the method in which measurements were made likely suffer from sampling errors induced by the digital structure of the amplitude spectrum. On the other hand, some studies (e.g., Baddeley \& Hancock, 1991; Hancock, Baddeley, \& Smith, 1992; Keil \& Cristóbal, 2000) report a greater bias for horizontal relative to vertical content. Of course, no definitive answer for all natural scenes can be determined as natural scene composition varies and the extent to which horizontal and vertical content differs within any given sample will depend on the specific environments in which the imagery is gathered. However, in a recent study by Hansen and Essock (2004a), support is given to the argument that a larger bias of horizontal content relative to vertical content for typical or modal outdoor scenes exists. To assess the anisotropy of natural scene content Hansen and Essock (2004a) gathered three different image sets (two from their lab and one from a different lab). The first set (Image Set 1) consisted of two-hundred thirty-one images (1024 x 1024 pixels) that were selected at random from their set of 1017 images described earlier, with the one stipulation that an equal number of scenes were selected from each annual season and contained only purely 'naturalistic' content.

The second set of imagery was obtained from a widely used and well calibrated image database compiled by a different lab (http://hlab.phys.rug.nl/archive.html, see van Hateren and van der Schaaf (1998) for detailed information about this imagery) for the purpose of an independent confirmation of the results from Image Set 1. Two-hundred 
images were randomly selected from this database to form the second image set (Image Set 2) which resulted in a variety of scene types. The random sampling process was conditioned so that only imagery devoid of man-made content would be selected. Given that this imagery is currently made available in 21 sets of 200 images, random sampling was also conditioned on sampling ten images per set (excluding set 1401-1600 as it consisted only of images of man-made content).

The third image set (Image Set 3) utilized by Hansen and Essock (2004a) was gathered in order to provide a highly detailed analysis of the distribution of the amplitude across orientation and spatial frequency. As mentioned earlier in the current section, some previous reports have attempted to provide a detailed measurement of amplitude within very narrow orientation bands (e.g., $5^{\circ}$ sectors) as a function of spatial frequency. However, a fundamental problem with such approaches is that, due to the discrete sampling of the digital Fourier transform, very narrow orientation band sectors centered at orientations other than $0^{\circ}, 45^{\circ}, 90^{\circ}$, and $135^{\circ}$ will not sample from the lower range of spatial frequencies. Specifically, a continuous Fourier transform will produce an amplitude spectrum, that, when plotted in polar coordinates, will yield a vector for each possible orientation, with each point on a given vector representing amplitude at a specific spatial frequency at that given orientation. However, due to the discrete representation of the amplitude spectrum (produced via the Discrete Fourier transform), not all orientation vectors can be represented across the full range of spatial frequencies (with lower spatial frequencies being most underrepresented). Only the vectors at the nominal orientations mentioned above posses amplitude coefficients across the full range 
of spatial frequencies produced by the digital Fourier spectrum, with many orientations not having any of the lower spatial frequencies represented. A procedure that sums along orientation vectors within a specific segment (spatial frequency range) is the best that can be achieved but will strongly bias amplitude measurements thereby yielding underestimates at orientations other than the four mentioned. The problem with such an approach is that one is left with only a measurement of amplitude for different spatial frequencies at four orientations. Thus if one required samples at orientations other than those four orientations, those vectors would have to be 'aligned' with that content. For example, if one wished to measure the distribution of amplitude across a full range of spatial frequencies at, for example, $3^{\circ}$ in an image, the spatial content of that image would have to be sampled in a way such that it would be plotted along one of the four nominal vectors in the Fourier amplitude spectrum. One way to achieve this, although time intensive, is to physically rotate the imaging device such that the spatial content at $3^{\circ}$ would be depicted along one of the ideal vectors mentioned above. The latter approach was exactly what Hansen and Essock (2004a) took in their analysis. Image Set 3 consisted of 60 natural scene images that were obtained in areas free of any carpentered structures. For each of the 60 scenes, the camera was rotated in $3^{\circ}$ steps, which resulted in 31 images per scene (refer to Hansen \& Essock, 2004a for details concerning the apparatus that allowed for the rotation of the camera). The sampling procedure resulted in a sampling of the same scene rotated in $3^{\circ}$ counter-clockwise steps. The 30 rotations (not counting the first sample - i.e., the aligned image) allowed for the utilization of two of the four optimal vectors mentioned above for a complete sampling of orientation across the range of $0^{\circ}$ to $180^{\circ}$ in steps of $3^{\circ}$ (with $0^{\circ}$ and $180^{\circ}$ being identical 
measurements of the same orientation, in this case vertical). That is, across the 31 images for a given scene, the $0^{\circ}$ vector (see Figure 8) in the Fourier domain allowed for the measurement of spatial content in the range of $90^{\circ}$ to $180^{\circ}$ (again at $3^{\circ}$ steps - i.e., $90^{\circ}$, $93^{\circ}, 96^{\circ} \ldots . . .180^{\circ}$ ), and the $90^{\circ}$ vector allowed for the measurement of spatial content in the range of $0^{\circ}$ to $90^{\circ}$.

For Image Sets 1 and 2, ratios indicating the magnitude of the orientation biases in the imagery were obtained for each image at each orientation based on the relative amount of the image's total amplitude contained in each of four $45^{\circ}$ orientation bands (at all spatial frequency bands - refer to Figure 9). Orientation was defined clockwise from vertical (i.e., vertical $=0^{\circ}$ ). The oriented content-bias of an image for a particular orientation was defined as the percentage of an image's total amplitude that was contained in a $45^{\circ}$ band (centered at $0^{\circ}, 45^{\circ}, 90^{\circ}$ or $135^{\circ}$ ) (i.e., the ratio between the summed amplitude contained within a $45^{\circ}$ sector and the value obtained from summing across the entire amplitude spectrum). For Image Set 1, three categories of images (15 images each) were defined and selected from the 231-image random sample; the three categories were defined on the basis of either containing a dominant horizon line, only the ground plane (i.e., various textures varying with season), or neither (e.g., images of general foliage, shrubbery, etc.). In addition, a fourth category of 186 images was created by removing all images from Image Set 1 that contained a predominant horizon line or receding ground plane (i.e., creating a set without any of the obvious spatial content presumed to create a horizontal bias). These four categories were then analyzed in terms of oriented content as described earlier. For Image Set 3 two analyses were carried out, first, overall magnitude 
(collapsed across spatial frequency) was obtained by averaging all of the amplitude coefficients along the respective vector for each of the 61 orientations sampled up to the Nyquist limit of the imagery (i.e., 512 cycles per picture). Second, in order to examine orientation biases as a function of spatial frequency, each orientation's respective vector was parsed into 20 bins (the maximum allowed by the Nyquist limit of this imagery), with each bin's amplitude coefficients being summed in order to provide a measure of amplitude contained at each of the 61 sampled orientations for each cycle per degree, ranging from $1 \mathrm{cpd}$ to $20 \mathrm{cpd}$.

The results of analyses of Image Sets 1 and 2, as shown in Figure 10, demonstrated that: (1) horizontal physical content indeed predominates in horizon-containing images, (2) horizontal content predominates even in non-horizon-containing scenes composed of ground surfaces, hillsides, or other regions consisting of similar vegetation or structure, (3) horizontal content predominates in a sample of scenes that contain neither a horizon or ground plane (such as close-ups of bushes, brush or general foliage), (4) horizontal structure persisted in dominating the analysis even when all imagery containing a receding ground plane or predominant horizon line were removed from the image sample, (5) a horizontal content bias was also found in an alternative set of "standardized" calibrated imagery frequently used in natural scene analysis (van Hateren \& van der Schaaf, 1998), and (6) there is a suggestion of a predominance of horizontal content evident in certain prior published reports (Baddeley \& Hancock, 1991; Hancock, Baddeley, \& Smith, 1992; Keil \& Cristóbal, 2000). Secondly, Hansen and Essock (2004a) note that the results of their analyses show that the vertical orientation (i.e., the 
$45^{\circ}$-wide bin centered on vertical) is second most prominent in typical scenes in our sample as well as in the large random sample taken from the imagery of van Hateren \& van der Schaaf (1998), also consistent with previous reports (Switkes, Mayer, \& Sloan, 1978; Van der Schaaf \& van Hateren, 1996; Keil \& Cristóbal, 2000; Oliva \& Torralba, 2001; Baddeley \& Hancock, 1991; Craven, 1993; Coppola, Purves, McCoy, \& Purves, $1998)$.

Since the analyses described above involved ratios of the summed amplitude coefficients in a $45^{\circ}$ wedge centered at each of the four primary orientations to the summed amplitude of the entire spectrum, it cannot be determined from the data just how the distribution of amplitude at orientations at or near the nominal orientations contributes to their respective biases at specific orientations or across spatial frequency. However, for reasons discussed earlier, the analysis carried out on the camera rotation imagery (i.e., Image Set 3) allowed for a more continuous measurement of amplitude at numerous orientations as a function of spatial frequency. These data (Figure 11) clearly show that there is a bias in summed amplitude at and near $90^{\circ}$ (horizontal content) that indicates more horizontal content relative to the other orientations. Figure 12 plots the averaged amplitude for each cycle per degree in the range of spatial frequencies allowed by the Nyquist limit of this imagery. There is a clear bias in amplitude at the cardinal orientations at each spatial frequency. Horizontal content is the most prevalent at all spatial frequencies. The second most prevalent content is always at vertical, although its prominence diminishes at the highest spatial frequencies. 


\section{HUMAN PERCEPTUAL PERFORMANCE AND THE CONTENT REGULARITIES OF NATURAL SCENES}

The following sections review the psychophysical experiments that have examined the extent to which human visual performance is guided by the spatial scale and orientation content regularities described in the earlier sections. In the sub-sections of Section 2.1, the relationship between the value of $\alpha$ of natural scenes and three aspects of human visual processing will be examined. The first aspect of visual processing will deal with adaptation to natural scene and natural scene-like imagery and its subsequent effects on visual sensation. The second will involve the ability of human observers to detect, or discriminate, a change in the spectral $\alpha$ of different natural scenes. Finally, the ability of human observers to discriminate between two different objects or natural scene images that have been progressively blended (e.g. object of image ' $\mathrm{A}$ ' is gradually "morphed" or "spectrally blended" into the object in image ' $\mathrm{B}$ '), with the ability to discriminate such changes under conditions where $\alpha$ was varied will be considered. In the sub-sections of Section 2.2, recent work from our laboratory will be reviewed where human visual perception of orientation was measured in the context of broad-scale visual noise in which $\alpha$ was varied (Section 2.2.1), as well as in the context of natural scenes containing content biases at different orientations (Section 2.2.2). 


\subsection{Human Perceptual Performance and Amplitude Spectrum Slope}

\subsubsection{Broad-scale Visual Adaptation of Natural Scene Imagery}

Before discussing visual adaptation, it is necessary to first discuss some aspects of human visual sensitivity as defined by the contrast sensitivity function or CSF. Simply put, the CSF is a plot of the minimum contrast required for a pattern to be detected. Contrast sensitivity varies as a function of spatial frequency of the test grating, resulting in a CSF that is band-pass in nature with a peak at about 3-6cpd and a cut-off of about $60 \mathrm{cpd}$ at $100 \%$ contrast. Adaptation experiments first demonstrated that the CSF is not due to a single sensitivity mechanism, but rather is the 'envelope' of multiple, relatively independent, narrowly tuned mechanisms (Campbell, Kulikowski, \& Levinson , 1966; Pantle \& Sekuler, 1968). That is, by adapting an observer to a pattern of a single spatial frequency, sensitivity will be reduced at a relatively narrow band of spatial frequencies rather than at all spatial frequencies. Similarly, the demonstration of these spatial frequency 'channels' was soon followed by the demonstration of narrowly tuned channels for different orientations (Blakemore \& Campbell, 1969; Blakemore \& Nachmias, 1971). When considering natural scenes as stimuli, the singlefrequency/single-orientation studies raise the important question regarding the effects of prolonged viewing of natural scenes on the visibility of the various components of natural scenes. Specifically, what affect does the distribution of content (scale and orientation) in typical natural scenes have on the sensitivity to single image components? 
Webster and Miyahara (1997) investigated whether or not prolonged viewing of the $1 / f^{a}$ distribution of spatial content of natural scenes (Figure 3d) alters sensitivity to individual sinusoidal gratings. The authors set out to investigate this question with a set of natural image stimuli ranging from open-range scenes to close-up scenes containing no man-made structure, as well as a set of visual-noise images possessing amplitude spectra similar to natural images (i.e., containing $1 / f^{\alpha}$ spectral slopes). The visual-noise patterns that were used consisted of six different spectral slopes ranging from $\alpha=0.0$ to $\alpha=-1.5$, in steps of -0.5 . The general procedure involved an initial five minute adaptation period where the subjects viewed a rapid and random succession of either natural scenes or noise patterns (for the noise pattern conditions, $\alpha$ was constant, with each $\alpha$ was tested separately). Following adaptation for a particular image type, observers engaged in a suprathreshold matching task that utilized sinusoidal gratings across a broad range of spatial frequencies. The results were quite striking in that they revealed CSFs with their peaks shifted to higher spatial frequencies for the natural scene stimuli. Given that the $\alpha \mathrm{s}$ of the natural scenes that were used were quite similar, there were no apparent differences in the shifting of the adapted CSF peaks for the different scene categories. Considering the large amount of amplitude present at low spatial frequencies, it makes sense that adaptation to such scenes would shift sensitivity away from the lower to mid frequencies. Even more interesting were the CSFs obtained after adaptation to the noise patterns with different $\alpha$, in that peak sensitivity for the adapted CSFs seemed to shift to higher and higher spatial frequencies as $\alpha$ increased, with virtually no change in the position of the peak for the patterns with $\alpha=0.0$. 
Thus, the traditional, unadapted, CSF does not characterize an observer's sensitivity to spatial content contained in a natural scenes in typical viewing conditions. Just as the bias in the distribution of natural scene content across spatial scale biases sensitivity away from the most prevalent content. Similarly, recent work (e.g., Essock, DeFord, Hansen, \& Sinai, 2003; Hansen et al., 2003; Hansen \& Essock, 2004a; 2004b) has shown that the orientation bias contained in natural scenes biases the visibility of oriented spatial content at the orientation of the most prevalent content in a given natural scene. When considered concurrently, human sensitivity to the specific content of natural scenes is adjusted by both the scale and orientation of content regularities typically found in natural scenes.

\subsubsection{Detection and Discrimination of Changes in the Spectral Slope of Natural Scenes}

As mentioned earlier in this review, the typical $\alpha$ for any typical natural scene is approximately 1.0. This can be seen in the sampling distribution (i.e., Figure 6b) obtained from randomly sampling natural images from the larger sample of naturalistic images mentioned earlier. Each point on those curves represents the number of orientation-averaged amplitude spectra slopes divided into smaller ranges. As can be clearly seen, the majority of images have an $\alpha$ value within the 0.80 to 1.0 range. Such a plot could be thought of as indicating the number of times one might encounter natural scenes with any particular $\alpha$ for any given day. From that sampling distribution, the likelihood of encountering scenes that deviate far from the 0.80 to 1.0 range is fairly small. This point has been made by a number of studies that have examined human visual performance in the context of natural scenes, in which it was argued that, since we 
are more likely to encounter environments exhibiting these $\alpha$ s, our visual systems should function optimally in such environments. The idea here is that during early development (and also long-term development on an evolutionary time scale), such image statistics were likely present and thus, over time, our visual systems were "wired" in a manner best suited to process visual information in the form of the typical $1 / f^{\alpha}$.

Knill, Field, and Kersten (1990) first set out to examine whether or not our visual systems might be optimally suited to process complex imagery at any given $\alpha$. In their study, Knill et al. used visual noise stimuli with varying $\alpha$ s where observers were engaged in a $2 \mathrm{AFC}$ task in which they were to detect a change in spectral slope. Technically, this task can be considered a detection task if one considers the standard noise pattern as the pedestal from which a change in $\alpha$ is detected. The results revealed that observers were best at detecting a change in $\alpha$ when the standard noise pattern was in the range of 1.4 to 1.8 . It was concluded that the human visual system is "tuned" to better process images with these $\alpha$ values. However, Tadmor and Tolhurst (1994) questioned these results since the range that was proposed to be best in terms of visual processing was well outside the typical range one might encounter; they also pointed out that while the noise patterns had spectral properties similar to natural scenes, they were still not representative in their content. Particularly, Tadmor and Tolhurst (1994) were interested in determining whether or not the human visual system was best suited to process information in the form of the typical $1 / f^{\alpha}$ relationship. They gathered two sets of imagery consisting of either natural scenes (with $\alpha$ s ranging from 0.78 to 1.38 ) or visual noise patterns with a comparable range of $\alpha$ values. The task they utilized was a 
spatial 3AFC, where two of the images (either natural scenes of visual noise) were set to one of seven reference $\alpha$ s and the other had a small change in $\alpha$, the observers were to identify which of the three was the "odd one out". The obtained results for noise and natural scenes were quite similar to the results obtained from the Knill et al. study; lower thresholds for steeper $\alpha \mathrm{s}$, with the highest thresholds obtained for reference $\alpha$ s near 1.0. The authors interpreted this finding as supporting the fact that the human visual system is optimally suited to process images exhibiting $\alpha$ s in the range of 0.8 to 1.0 . This interpretation may seem odd since thresholds were highest in this range, but consider what a high threshold for discriminating changes in $\alpha$ implies. Tadmor and Tolhurst (1994) argue that such a high discrimination threshold for reference images in the typical $\alpha$ range suggests a high degree of "tolerance" for changes in $\alpha$ that might occur within this range. They reason that since we typically encounter scenes with $\alpha$ near 1.0 , slight deviations from this value that might occur as a result of, for example, shifting our gaze from one area to another within a given environment, or slight accommodative errors due to fixating objects at different distances, and it would be beneficial if this had little impact on our ability to effectively process visual information. Such a tolerance allows us to view the visual system as being set up to "expect" small deviations from the typical $\alpha$ and therefore not waste valuable processing time and resources by signaling to the observer that such changes have occurred. It is in this sense that higher thresholds for images with typical $\alpha$ values can be considered optimal.

Another aspect to consider is that the reference $\alpha$ s that were used as the comparison from which the changes in $\alpha$ were to be discriminated were not the true $\alpha$ s of the original 
images (also the mean luminance and r.m.s contrast were constrained to very low values). This begs the question of whether or not these results would be obtained if observers were to discriminate changes in $\alpha$ relative to the true $\alpha$ s of natural scenes. Thomson and Foster (1997) addressed this question indirectly while examining the role of the phase spectra of natural scenes in detecting changes in $\alpha$. The task was a temporal 2AFC that utilized natural scenes ranging from texture patterns to actual scenes. For each $\alpha$ category tested $(0.7,1.0,1.3)$, thresholds were highest when the natural image's $\alpha$ was closest to its original value. This seems to argue against the idea of an ideal $\alpha$ about which our visual systems are most tolerant, although it should be noted that the extreme images (0.7 and 1.3) Thomson and Foster (1997) used were rather atypical, consisting primarily of texture patterns (utilized to address a different issue). Their findings are still compelling, and call for a more extensive, highly controlled investigation of this issue.

\subsubsection{The Role of Spectral Slope in Discriminating Between Different Scene Content}

In the previous section, the question of interest was whether or not the human visual system is tuned to optimally process natural scenes that have the characteristic $\alpha=1.0$; and, with the caveat stated, it appeared as though it was. One interesting aspect from the Tadmor and Tolhurst (1994) paper was the rating that participants gave to the highly constrained imagery when presented at different reference $\alpha$ values. Participants were asked to subjectively rate the images in terms of "best perceptive quality," that is, at which reference $\alpha$ did the images appear as having the best quality (these images were considerably "degraded" in terms of having low r.m.s contrast and mean luminance). Regardless of the original images' true $\alpha$ s, all images were rated to have the best 
perceptual clarity when the reference $\alpha$ was set to roughly $\alpha=0.8$. The implication here is that when imagery is of sub-standard quality, the $\alpha$ that yields highest perceptual quality was close to the typical $\alpha$ of natural scenes. It is at this $\alpha$ value (when scenes are highly constrained) that people might expect to best perform some visual task. One of the many assumptions here is that when adjusting degraded imagery by some factor ( $\alpha$ in this case), observers might be using an internal criterion that could be associated with performing various tasks that require images to be of the best quality. This raises the issue of whether or not there is an ideal $\alpha$ under which people can best perform some visual task. Párraga, Troscianko, and Tolhurst (2000), and Tadmor and Tolhurst (2000) addressed this issue with two unique visual discrimination tasks that would provide some potential answers.

The task that was devised by Párraga et al (2000) involved progressively "morphing" two natural images that were very different in perceptual meaning (e.g. car and bull or the face of a man and the face of a woman), but had similar salient features. In a modified $2 \mathrm{AFC}$ procedure where three images were presented sequentially (with the second image always being the unchanged reference image), observers were instructed to indicate which interval $\left(1^{\text {st }}\right.$ or $\left.3^{\text {rd }}\right)$ contained the stimulus image that was different from the reference ( $2^{\text {nd }}$ interval) image. All three images were set to a single $\alpha$ value. Threshold was considered the amount of morphing (applied in progressive steps) that occurred in the test image that allowed observers to discriminate a change. The results indicated that observers were best able to discriminate when an image was being morphed into another image when $\alpha$ was close to 1.0. Tadmor and Tolhurst (2000) had conducted a similar 
study where the Fourier spectra (amplitude and phase) of two different images were spectrally blended in progressive steps. Again it was found that observers had the lowest discrimination thresholds when the reference $\alpha$ was near 1.0. However, lower thresholds around $\alpha=1.0$ were not clearly observed when the imagery was highly constrained in the manner discussed earlier; instead discrimination thresholds were lowest across a much broader range. Taken together, the studies mentioned above provide some evidence that the human visual system is best suited to discriminate spatial form when in the context of natural scene imagery possessing the typical $\alpha$ that characterizes natural scenes.

\subsection{Human Perception of Orientation in Broad-Scale Stimuli}

In the preceding sections, evidence was presented in support of optimal visual processing of real-world content when the imagery possessed isotropic (i.e., orientation averaged) amplitude spectra with a fall-off best described with an exponent approximately equal to 1.0 (in logarithmic coordinates). However, since the stimuli described in the preceding sections were made to possess isotropic spectra, the dimension of orientation was not considered. As mentioned earlier in the introduction, typical natural scenes show a persistent bias in amplitude at the cardinal orientations, thus the same line of reasoning that argued for the visual system to be optimized to process $\alpha=1.0$ imagery, would also argue that the visual system my show processing strategies that match the orientation bias of natural scenes. In fact, the existence of the orientation bias has lead many researchers to propose that this bias could very well provide an evolutionary/developmental explanation for the well documented "oblique effect" (i.e., poor visual sensitivity for obliquely oriented stimuli). Specifically, it has been posited that due to the relative lack 
of content at the oblique orientations, the development of our visual systems (either ontologically or phylogically) would have been biased to process content at the more prevalent orientations. Recently, there have been a few studies that considered this issue by examining human visual processing of differently oriented stimuli 'embedded' in broad-scale stimuli. Surprisingly, the results of these studies show that human visual processing of orientation in natural and natural-like stimuli is best for obliquely oriented stimuli, worst with horizontally oriented stimuli, and intermediate for vertically oriented stimuli (i.e., a "horizontal effect"). However, before moving the discussion towards the results of those experiments, it is first necessary to briefly review the literature devoted to investigating the oblique effect along with the considerations for the underlying causes of such an effect.

\subsubsection{Human Perception of Orientation: The Oblique Effect}

\subsubsection{The Behavioral Oblique Effect}

It is well established that visual sensitivity varies as a function of stimulus orientation.

There is a long history of research devoted to examining how human (e.g., Jastrow, 1893) and animal (e.g., Lashley, 1938) performance on a variety of tasks varies with stimulus orientation. The typical finding is that performance is superior for horizontal and vertical orientations (cardinal axes), relative to $45^{\circ}$ and $135^{\circ}$ oblique orientations (oblique axes). This general perceptual bias in visual processing has been labeled the oblique effect (Appelle, 1972). However, it is important to realize that there are two different sources of anisotropic "oblique effect" behavioral performance: an orientation bias in the basic 
functioning of the visual system (e.g., contrast sensitivity and acuity), and a bias of later visual processing, presumably beyond V1 obtained on more cognitive tasks such as the naming, encoding, and memory of orientation, in which oblique orientations are more confused with each other than are stimuli of cardinal orientations. To help avoid confusing these two oblique effects in the literature, they have been termed class 1 and class 2 oblique effects, respectively, distinguishing them on the basis of the type of task on which they occur (Essock, 1980). That the two types of oblique effect are fundamentally different, is readily apparent by considering that the bias of contrast sensitivity is fixed to retinal coordinates if the viewer's head or body is rotated, but that the oblique effect of orientation memory is often labile, dependent upon the observer's sense of "up". Furthermore, the memory anisotropy is readily obtained with non-visual stimuli, whereas the class 1 oblique effect seems closely tied to a physiological bias in the early visual cortical area. For the remainder of this paper, any mention of the oblique effect will be in reference to the first class; specifically in terms of overall orientation sensitivity to simple stimuli such as lines or high spatial frequency gratings, its neurophysiological basis, its evolution and its relation to the visual anisotropy demonstrated to exist in the context of visual processing of natural scenes (i.e., the horizontal effect).

\subsubsection{The Physiological Oblique Effect}

In addition to behavioral studies, physiological research has shown differences in the relative numbers of oriented receptive fields sampled in V1 tuned to cardinal axes orientations relative to oblique axes orientations (DeValois, Yund, and Hepler 1982; 
Mansfield, 1974; Orban \& Kennedy, 1980; Li, Peterson, \& Freeman, 2003), and the related measures of the amount of regional electrical response (Mansfield and Ronner, 1978 ) or the amount of cortical area activated by that orientation (Chapman, Stryker, \& Bonhoeffer, 1996; Chapman \& Bonhoeffer, 1998; Coppola, White, Fitzpatrick and Purves, 1998). In humans, the evidence of a neurophysiological oblique effect bias consists of VEP amplitude and latency (Sokol, Moskowitz, \& Hansen, 1989; Zemon,Gutowski, \& Horton 1983; Maffei \& Campbell, 1970; Yoshida, Iwahara, \& Nagamura, 1975) as well as fMRI neuroimaging magnitude (Furmanski and Engel, 2000). Since threshold for a particular stimulus pattern is thought to be directly associated with the number of neurons tuned to that pattern (Essock, Krebs, \& Prather, 1997; Anzai, Bearse, \& Freeman, 1995 ), this numerical anisotropy in the tuning preferences of neurons would explain the presence of the behavioral oblique effect. Another physiological bias is that neurons with preferred orientations at a cardinal axes show narrower orientation tuning (Rose \& Blakemore, 1974; Orban \& Kennedy, 1980), as do human orientation channels (Campbell, Kulikowski, \& Levinson, 1966), but this could very well be a secondary trait resulting from the numerical bias creating a greater inhibition pool ("intra-channel inhibition") from the more numerous units.

\subsubsection{Origins of the Oblique Effect}

While considerable progress has been made in understanding the cortical underpinnings of the oblique effect, it remains unclear as to why such a bias should exist in the visual system. As mentioned at the beginning of the current section, many researchers have taken an ecological approach in theorizing about the origins of the oblique effect, noting 
the preponderance of horizontal and vertical content in the natural (or man-made) visual environment and suggest that either through ontogeny or phylogeny, a neural bias exists in response to the environmental orientation bias (e.g., the predominant horizon and vertical vegetation growth; see Section 1.2). Some, such as Annis and Frost (1973), have pointed to visual deprivation studies that demonstrate a neural plasticity where visual experience with exclusively, or presumably predominately, one stimulus orientation results in a distribution of neurons' preferred orientation in which the observed orientation predominates. They suggest that the neural oblique effect bias is a consequence of being reared in a "carpentered world". Others, such as Timney and Muir (1976), have suggested that the basis of the neural oblique effect is a genetic bias in the distribution of preferred orientations that is independent of early experience. Here the suggestion is that the bias has evolved in humans to neurally emphasize the coding of the most prevalent content in the natural environment. Timney and Muir's (1976) additional conclusion that the genetically determined bias varies in magnitude between certain ethnic groups also accounts for the findings of Annis and Frost (1973). Held, Thorn, McLellan, Grice, and Gwiazda (2000) proposed an alternative view that holds that the basis of the oblique effect is indeed a genetic bias, and varies in magnitude between groups of Caucasian and Asian ancestry, but that it leads to a neural bias due to the effects of early astigmatism.

While such arguments in favor of either "nature" or "nurture" are often futile at best, there does exist considerable evidence that argues more strongly for the "nature" view over a strict "nurture" view. First, a careful examination of the orientation deprivation 
studies shows that the most parsimonious account of the obtained distribution of preferred orientations is that these are a subset of neurons that are intrinsically tuned to horizontal or vertical orientations and are not modified by early visual experience. These are observed even in the presence of highly-unnatural restricted conditions that indeed bias the tuning of many neurons (Blakemore \& Cooper, 1970; Hirsh \& Spinelli, 1970; Hubel \& Wiesel, 1970; Leventhal, \& Hirsch, 1977; Frégnac, \& Imbert, 1978; Sengpiel, Stawinski, \& Bonhoeffer, 1999). In addition, recent results (Chapman, Stryker, and Bonhoeffer, 1996) from optical imaging studies show good evidence that, at least in ferret, the orientation columns expressing a horizontal or vertical bias develop first (and are immutable regardless of visual depravation), but are of equal width, and with normal experience the horizontal and vertical orientation columns become wider than oblique columns, presumably containing more neurons tuned to cardinal orientations. Thus, it seems that a pre-programmed numerical oblique effect bias occurs due to this genetically programmed subset of neurons, with the potential for reinforcement of this bias occurring through visual experience in environments biased at the cardinal orientations.

Given the above considerations on the origins of the oblique effect, numerous researchers have drawn parallels between the structural anisotropy observed in real-world imagery and the behavioral oblique effect measured psychophysically, with the implication being that the structural bias my serve as a potential origin of the oblique effect performance bias. Aside from the evidence discussed above, there exists a limitation in most of the discussion of the development and basis of the oblique effect that is repeatedly ignored. It is that the behavioral (i.e., class 1 ) oblique effect is 
invariably tested with simple line, edge or grating stimuli. Such simple visual stimuli presented in isolation are quite unlike the visual information humans are actually presented in their real visual environments. In other words, the class 1 oblique effect is demonstrated with very simple, rather non-naturalistic stimuli such as high spatial frequency gratings, whereas our natural environment is highly broadband (or broadscale), containing energy at all spatial frequencies and orientations. Accordingly, human visual sensitivity to differently oriented stimuli would need to be tested in the presence naturalistic content in order to determine whether such an effect is still present. It is this latter issue that will be the focus of the remainder of the current section.

\subsubsection{Human Visual Perception of Oriented Content in Broadband Noise}

In a series of psychophysical studies, Essock et al. (2003) sought to determine whether or not the oblique effect would be present with stimuli that possessed broadband structure. The fundamental drive behind their experiments was to examine the abilities of human observers to accurately perceive or detect the presence of oriented content in the context of broad-scale structure representative of natural scenes (i.e., perceivable energy at all spatial frequencies and orientations). The primary problem that had to be overcome concerned first, how to generate stimuli that resembled natural scenes, while at the same time offered a respectable amount of control when it came time to interpret the data. The second issue had to do with how to add oriented content to the patterns at specific orientations, while at the same time assuring that the distribution of the added oriented content also resembled that typically found in natural scenes. The first issue was resolved with the use of broadband visual noise patterns that had been used in previous 
studies designed to investigate human processing with respect to changes made to $\alpha$ (Knill et al., 1990; Webster \& Miyahara, 1997; Thomson \& Foster, 1997). The use of such patterns allowed the authors to construct broadband patterns in the Fourier domain that could be made to have any desired slope, while being absent of any meaningful semantic content that might result in potentially misleading results. The second issue was addressed by constructing a broadband triangle weighted filter in the Fourier domain. This filter allowed Essock et al. (2003) to make increments to the amplitude spectra of different noise patterns in a way that resembled how amplitude biases are typically observed to occur in natural scenes (i.e., the biases are typically peaked at whatever orientation possesses the bias). Thus, oriented biases of amplitude could be added to a given noise pattern with the peak centered on the orientation at which sensitivity would be tested. In this particular study, Essock et al. (2003) utilized three psychophysical paradigms to examine human visual processing of broadband oriented content embedded in broadband visual noise patterns. The paradigms involved three levels of human sensitivity including a suprathreshold matching paradigm (method of adjustment), a temporal 2-AFC (method of constant stimuli) threshold paradigm, and a near-threshold single interval Yes/No paradigm.

For the suprathreshold paradigm, several sets of stimuli were constructed in the frequency domain and made to have amplitude spectra with $a$ values of $0.0,0.5,1.0$, and 1.5 (see Figure 13 for examples of these patterns). The phase spectra of the stimuli were randomly assigned values ranging from $-\pi$ to $\pi$, thus the corresponding spatial 'patterns' 
did not possess any meaningful spatial content. Again, the reasoning behind utilizing visual noise patterns was two-fold: first, to examine visual perception of orientation purely as a function of the slope of the amplitude spectra, and second, to avoid any possible interaction with the information 'carried' by the phase spectra (e.g., the semantic meaning). In order to 'embed' broadband oriented structure in these stimuli, a triangle weighting function (with an orientation bandwidth of $45^{\circ}$ ) was multiplied with the amplitude spectra (centered at each one of four different orientations: vertical, $45^{\circ}$ oblique, horizontal, and $135^{\circ}$ oblique). Thus the oriented content present in the stimuli was solely defined in terms of oriented amplitude increments. Participants were seated in front of a monitor covered by a large circular mask with two circular, laterally displaced windows in which two noise patterns were presented (see Figure 14a). On the left was a comparison pattern that contained a fixed suprathreshold oriented increment of amplitude (at either $22.5^{\circ}$ or $112.5^{\circ}$ ), and on the right was a test pattern (with an oriented increment of amplitude at one of the four nominal orientations) in which observers were instructed to adjust (via key-press) the magnitude of the content until it appeared to match that contained in the comparison pattern. As mentioned in earlier sections, the data clearly demonstrated that matches were most accurate for oblique stimuli, least accurate for horizontal, and intermediate for vertical; an effect the authors termed the "horizontal effect" (see Figure 14b, and Figure 14d for an example of the effect itself). This effect was found to occur in all $\alpha$ conditions, however, the magnitude of the effect was maximal in the condition where $\alpha$ was equal to 1.0 (see Figure 14c). 
For the temporal 2-AFC threshold paradigm, the same methodology for creating the stimuli was used as described above, only the magnitude of the increment varied from $2 \%$ peak increment to $24 \%$ peak increment in steps of $4 \%$ and only noise patterns with an $\alpha$ equal to 1.0 were used. The procedure itself was blocked by orientation (i.e., participants knew which orientation they were looking for) and involved two successive presentations (separated by $500 \mathrm{msec}$ ) of either a noise pattern with an oriented amplitude increment or a noise pattern without an oriented increment. The task of the observers was simply to indicate which of the two intervals contained the oriented increment (via key-press). The results were very similar to those obtained in the suprathreshold matching paradigm in that sensitivity for detecting obliquely oriented increments of amplitude was best, with performance for detecting horizontally oriented increments of amplitude was worst and performance for detecting vertically oriented increments of amplitude was intermediate (see Figure 15a). For the near-threshold single interval Yes/No detection paradigm, only one increment level was utilized ( $20 \%$ peak increment). The task was similar to the 2-AFC paradigm, only instead of two stimulus intervals, one stimulus interval was presented. The task of the participants was to indicate whether or not the presented stimulus contained an oriented increment of amplitude or not (via keypress). Similar results to those of the threshold and suprathreshold paradigms were obtained (see Figure 15b).

Thus, the results reported by Essock et al. (2003) indicate that the perception of oriented content in broadband noise patterns with amplitude spectra mimicking that of natural scenes produces performance very different from that found with simple stimuli 
or that might have been predicted if performance is presumed to match the prevalence of oriented content in typical natural scenes (refer to Section 3.0 for further discussion).

\subsubsection{Human Visual Perception of Oriented Content in Natural Scene Stimuli}

Based on the results reported by Essock et al. (2003), an argument could be made that the results were specific to stimuli consisting of broadband visual noise, and that such an effect may not have been present if the stimuli had consisted of actual real-world scenes. Essentially, this argument reduces to whether or not the phase spectra would interact with the detection of oriented structure defined as broadband increments of amplitude. That is, since it is the phase spectra that specifies how the different sinusoidal components of an image's 'signal' sum to form a given scene, the amount and localization of the lines and edges that form the meaningful content are determined by each scene's phase spectrum. Whether or not the presence of the phase defined content ${ }^{1}$ of an image would produce the same effect observed with visual noise patterns was investigated by Hansen et al. (2003) and Hansen and Essock (2004a; 2004b). These authors investigated human visual sensitivity for detecting oriented increments of amplitude with three different types of natural scene imagery. The following sub-sections are thus grouped with respect to experiments conducted with specific types of imagery which include: 1) imagery possessing large amounts of 'natural' content biases (trees, horizon, branches, etc.) at one of four orientations (summarized in Part I below), 2) imagery containing approximately equal amounts of natural content at all orientations, but possessing different $\alpha$ values

\footnotetext{
${ }^{1}$ For all intensive purposes, the term "content" speaks to the structural components of a given scene. For example, for a scene consisting of trees, the lines and edges that make up the perceived form of the trunks of the trees would be an example of the vertical content in that scene.
} 
(summarized in Part II below), and 3) imagery containing variable amounts of natural content biases at one of four orientations (summarized in Part III below).

\subsubsection{Visual Processing of Orientation in Natural Scene Stimuli: Part I}

In the studies conducted by Hansen et al. (2003) and Hansen and Essock (2004a) sets of imagery were gathered that contained naturalistic content biases at one of each of the four nominal orientations (vertical, $45^{\circ}$ oblique, horizontal, or $135^{\circ}$ oblique) as well as imagery containing equal amounts of naturalistic content at all orientations (all imagery had amplitude spectra $\alpha$ values approximately equal to 1.0 -- refer to Hansen et al., 2003 for a detailed description of the image sampling, correction, and selection procedures), see Figure 16 for example imagery. The primary question of those studies was to examine whether or not the horizontal effect would be observed with actual natural scene images as stimuli. Additionally, those studies were designed to assess whether or not the presence of content biases at different orientations (e.g., predominance of trees, presence of a horizon, branches at various angles, etc.) would have a generalized impact on the ability to detect increments of amplitude at different orientations. The oriented increments of amplitude were applied in the same manner as with the noise patterns utilized by Essock et al. (2003), only before weighting the spectra with the triangle filter, the amplitude spectra of the imagery were averaged across orientation in order to eliminate the content biases as defined by the original spectra. This resulted in maintaining the form (i.e., slope or $\alpha$ ) of the spectra while preserving the content biases as defined by the phase spectra of the imagery (refer to Figure 17 for further details). The experimental paradigm was a single interval Yes/No design where on any given trial, 
observers were required to indicate (via key-press) whether or not the stimulus image contained an oriented increment of amplitude - trials were blocked by orientation of the amplitude increment. The data are re-plotted in Figure 18a and show the presence of two performance biases. First, regardless of the content bias present in the natural stimulus images, performance for detecting horizontal increments of amplitude was always poor. Second, performance for detecting an oriented increment of amplitude was reduced when the oriented increment was at the same orientation as the phase-defined content bias (i.e., a "content-dependent effect"). In Addition, Hansen et al. (2003) showed that when the coordinates of the phase spectrum corresponding to the biased content in the spatial image (at any of the four orientations tested) was scrambled (thereby eliminating the presence of the content biases at any of those orientations), performance for detecting increments of amplitude at the previously content-biased orientations improved considerably (see Figure 18b).

\subsubsection{Visual Processing of Orientation in Natural Scene Stimuli: Part II}

In the study conducted by Essock et al. (2003), experiments were conducted where human perception of orientation in the context of broadband visual noise stimuli with amplitude spectra $\alpha$ values that ranged from 0.0 to 1.5 (in steps of 0.5 ) was found to be highly anisotropic. As shown in Figure 14, the magnitude of that anisotropy was higher when the $\alpha$ value of the noise was 1.0. However, as mentioned earlier, those stimuli had random phase spectra and thus contained similar amplitude spectra, but not the semantic content inherent in natural scenes. The findings reported by Hansen et al. (2003) and Hansen and Essock (2004a) did not address the ability to detect oriented increments of 
amplitude in natural scene imagery with variable $\alpha$ values, the focus of those studies was more on the existence of interactions between the phase-defined content biases of natural scenes and the ability to detect oriented increments of amplitude. Thus, the question of whether or not the ability to detect oriented increments of amplitude for real-world stimuli consisting of naturalistic content would depend on $\alpha$ was not addressed. However, this issue was addressed by Hansen and Essock (2004b) who carried out experiments that utilized sets of natural scene imagery that possessed a broad range of amplitude spectrum $\alpha$ values, but without any predominant 'natural' content biases (i.e., the imagery was naturally isotropic). Specifically, those authors sought to determine if the magnitude of the horizontal effect changed as a function of the $\alpha$ value of natural scenes as it did with the noise patterns used by Essock et al. (2003). In order to test this, Hansen and Essock (2004b) gathered sets of images that contained approximately equal content (amplitude) at all orientations, with each set containing images that had amplitude spectra $\alpha$ values within one of several narrow ranges (see Figure 19 for example imagery). Utilizing the same single interval Yes/No paradigm described earlier, the authors reported results that were consistent with those of the suprathreshold matching paradigm conducted by Essock et al. (2003). Specifically, a horizontal effect was observed in all $\alpha$ conditions, with the magnitude of the horizontal effect being highest for imagery that had $\alpha$ values closest to 1.0 (refer to Figure 20). However, an important distinction must be made here in that, while the salience of broadband horizontal content followed the slope of noise patterns, sensitivity for detecting horizontal increments appears relatively stable, with relative sensitivity to the other three 
orientations tested demonstrating the change in sensitivity (being best when a was approximately equal to 1.0 (c.f., Figure 20).

\subsubsection{Visual Processing of Orientation in Natural Scene Stimuli: Part III}

As mentioned earlier, typical natural scenes tend to possess content biases at the cardinal axes (with more content at horizontal relative to vertical), thus one is left with the question of whether or not the visual system is optimally suited to process orientation information in scenes that possess such biases. This issue was partially addressed in the work of Hansen et al. (2003) and Hansen and Essock (2004a), discussed in Section 2.2.2.1. In Figure 18, overall orientation performance is best in the imagery containing natural content biases at vertical and horizontal (the typical biases of natural scenes) relative to the set of images with natural biases at either of the oblique orientations. However, it is unclear from that figure whether or not overall orientation performance is best for imagery with natural horizontal biases compared to overall performance in the vertical content biased imagery. Hansen and Essock (2004b) sought to examine this issue by measuring human visual sensitivity with natural scene imagery containing variable amounts of content biases at each of the four orientations to determine if sensitivity for detecting amplitude increments at all orientations depended on the relative predominance of content at a given orientation for a given image. The idea here is that if the visual system is optimized to process orientation in the presence of the typical orientation bias found to occur in natural scenes (i.e., the horizontal bias), overall orientation performance should follow the magnitude of the horizontal bias (i.e., high overall performance with imagery containing large amounts of horizontal content and low overall performance 
with imagery containing relatively small amounts of horizontal content), and not biases at other orientations. In order to test this, Hansen and Essock (2004b) compiled a set of natural scene imagery to serve as experimental stimuli in which the amount of horizontal content (carried by the phase spectra) was variable. Additional control image sets were also obtained that contained variable content biases at the other nominal orientations (i.e., vertical, $45^{\circ}$ and $135^{\circ}$ oblique) refer to Figure 21 for examples. All experimental methods were similar to those described in Section 2.2.2.1. The results were that performance for detecting increments of amplitude at all tested orientations was significantly related to the amount of horizontal content present in the horizontal contentbiased image set, with no significant relationship to the amount of content-bias contained in the control image sets (see Figure 22). 


\section{TOWARD A STRIATE MODEL OF BROADBAND}

NATURAL SCENE CONTENT

The results described in Section 2.2.1 (i.e., Essock et al., 2003) emphasized that when performance for perceiving broadband increments of amplitude at one of the four nominal orientations was evaluated at threshold, near-threshold, and suprathreshold, a strong anisotropy that is very different from the oblique effect anisotropy (that occurs with grating stimuli) is obtained. That is, instead of targets at oblique orientations being seen most poorly, horizontal stimuli were seen most poorly and oblique stimuli were seen best, with vertical performance intermediate. This was true whether the amplitude spectrum mimicked the content of natural scenes or deviated considerably in terms of the relative content at the different scales of spatial structure. However, the magnitude of this effect did depend on the $\alpha$ of the broadband noise patterns' amplitude spectra, with the highest magnitude occurring when this value was set to 1.0. Similar results were obtained by Hansen and Essock (2004b - i.e., Section 2.2.2.2) where naturally isotropic natural scenes with varying as were used as stimuli. Thus, when compared to the perception of an isolated grating or line stimulus, the presence of additional spatial components in a visual stimulus results in interactions that strongly alter the relative visibility of oriented content at various orientations. That is, the oblique effect obtained with simple stimuli does not extend to naturalistic viewing situations as many have presumed. Specifically, a horizontal effect is obtained instead. When this effect was 
examined in the context of natural scenes that contained a natural bias in oriented content (containing predominant content at either $0^{\circ}, 45^{\circ}, 90^{\circ}$, or $135^{\circ}$ ), as well as with natural scenes that roughly contained an equal amount of content across orientations, the horizontal effect could still be observed. Additionally, when the orientation of the broadband increment matched the orientation of the content bias of the imagery, performance for detecting those orientation increments was dramatically reduced, thus suggesting the presence of an additional dynamic ${ }^{2}$ 'content-dependent' effect. Interestingly, while the magnitude of the horizontal effect was shown to be maximal when the $\alpha$ of the stimuli was close to the typical value encountered in natural scenes (i.e., being highest when the value was set to equal 1.0 [broadband visual noise patterns] or when it was very close to this value [as assessed with scenes that were allowed to maintain their original slope]). Thus, not only does visual performance for detecting/recognizing changes in object for appear to be maximal when $\alpha$ values are close to one (see Section 2.1.3), but broadband orientation sensitivity to horizontal increments also follows the $\alpha$ values. This relationship is also close to that observed where human sensitivity to slope changes (i.e., Section 2.1.2) exhibits higher thresholds when the slopes of the stimuli are close to 1.0. That the magnitude of the horizontal effect is highest for $\alpha \approx 1.0$ suggests that there might be an additional content regularity in natural scenes where larger amounts of horizontal content are found to exist in spectra where the slopes are close to 1.0. While our lab has looked for such a bias, there were not enough images in our sample set with either very shallow or very steep slopes to arrive at any clear conclusions, and currently, there are no other reports in the literature that have

\footnotetext{
${ }^{2}$ Dynamic in the sense that the presence of the content dependent effect will change with respect to which orientation in a given scene contains a predominant amount of content.
} 
found such a relationship to exist. Lastly, while the magnitude of the horizontal effect was shown to vary with the slope of the amplitude spectra, Sections 2.2.2.2 and 2.2.2.3 reviewed results that showed overall broadband orientation sensitivity, not only to relatively follow the slope of the amplitude spectrum, but also the relative amount of the horizontal content bias inherent in a given natural scene respectively.

The experiments described in Section 2.2.1 and the sub-sections of Section 2.2.2 support the existence of two general orientation processing anisotropies for broadband stimuli. The first, the horizontal effect, can be considered an inherent (or 'static') anisotropy in that, regardless of the slope of the amplitude spectrum or content biases at a given orientation, poor sensitivity for detecting horizontal increments of amplitude was always observed. The second, the 'content-dependent' effect, can be referred to as a more 'dynamic' anisotropy in that sensitivity for detecting broadband increments of amplitude at the other three tested orientations (i.e., vertical, $45^{\circ}$ oblique, and $135^{\circ}$ oblique) depended on the content biases of the natural scene stimuli selected as stimuli. Additionally, this second effect can be described as having three components: 1) when the orientation of the amplitude increment matched that of the content bias contained in natural scene stimuli, performance for detecting those increments was dramatically reduced with respect to conditions where no such content biases were present - from here on referred to as content-dependent effect 1,2) overall performance for detecting amplitude increments at off-horizontal orientations was relatively best when the slope of the amplitude spectrum resembled the typical slope found to occur in natural scene imagery (i.e., $\alpha \approx 1.0$ ) - from here on referred to as content-dependent effect 2 , and 3) 
overall performance for detecting amplitude increments at all four orientations depended on the relative amount of horizontal content contained within the natural scene stimuli from here on referred to as content-dependent effect 3 . The following sub-sections will speak directly toward possible cortical mechanisms that could account for the two types of orientation processing-anisotropies summarized above.

\subsection{The Inherent Orientation Processing Anisotropy: A Horizontal Effect}

The association between the behavioral performance horizontal effect observed with broadband natural stimuli and the prevalence of content at the nominal orientations in natural scenes was investigated by Hansen and Essock (2004a). That the content contained in typical scenes exhibit a horizontal bias is an important finding as those authors have proposed that the behavioral horizontal effect would have evolutionary utility in such environments (Essock et al., 2003; Hansen et al., 2003). Specifically, such a hypothesis predicts the existence of a cortical mechanism (presumably at the level of striate cortex) that acts to reduce the perceptual salience of the most prevalent content (i.e., horizontally oriented structures) in a scene, thereby enhancing the less often occurring content of natural scenes. That is, a mechanism that turns down sensitivity for the 'expected' content in a typical scene would serve to relatively enhance the salience of 'unexpected', or novel, content at off-horizontal orientations. This pattern of sensitivity adjustment could most likely be accounted for by some type of specialized cortical gain control mechanism. However, the change in the orientation sensitivity obtained with broad-spectrum stimuli cannot be expected from standard models of contrast gain control (e.g., Bonds, 1989; Heeger, 1992, Geisler \& Albrecht 1992; Wilson \& Humanski, 1993, 
Carandini \& Heeger, 1994). That is, typical models propose that the output of V1 cortical units is modulated by division of their response by the summed activity of other units pooled equally across all orientations and some (if not, then all) spatial frequencies; and thus assume equal amounts of activity amongst those cortical units. However, the experiments reviewed in the preceding sections indicate that the weights for various orientations contributing to the normalization pool are not equal and function to adjust more selectively with respect to orientation (i.e., only similarly tuned units would adjust the output of one another). Specifically, when any given broadband test pattern used in those studies was oriented obliquely, it would cause the gain to be turned down less than when it is oriented horizontally. Consistent with this proposal, numerous studies have indicated that among striate cortical neurons mediating central vision, horizontal and vertical preferred orientations are somewhat more prevalent than oblique orientations (Mansfield, 1974; Tiao \& Blakemore, 1976; Mansfield \& Ronner, 1978; Orban \& Kennedy, 1980; De Valois et al., 1982; Chapman et al., 1996; Coppola et al., 1998; Furmanski \& Engel, 2000, Li et al., 2003). Thus, when the output of the different units is pooled in restricted orientation (and presumably spatial frequency) ranges, the divisive signal would be weaker at oblique orientations, resulting in the observed stronger response at oblique orientations when viewing broadband patterns. In other words, when the horizontal orientations of the amplitude spectrum of any given broadband pattern are incremented, this would cause more total pooled activity at the horizontal test orientation than when oblique orientations are incremented, thus turning down the output of the units detecting the test pattern at horizontal more than when the pattern is at oblique orientations. Accordingly, such an adjustment would thereby produce a relatively smaller 
perceptual response for horizontally oriented content compared to obliquely oriented content in a broadband pattern.

\subsection{Dynamic Orientation Processing Anisotropies: The Content-Dependent Effects}

Considerable research has shown that when differently oriented simple stimuli (e.g., sine waves) are presented simultaneously, a contrast normalization mechanism alters the sensitivity to a test stimulus. Certainly viewing natural scenes with their broad spatial scale and broad orientation content also evokes contrast gain adjustments. As mentioned in the preceding section, typical models of contrast normalization assume pooling of the output of an array of linear filters of varied spatial frequency and orientations. Most models suggest that the current image (or "recent" image due to a delay in the mechanism) is filtered by the array of filters (or a subset in certain models) and that their responses to the current/recent stimulus is pooled in a normalization pool that alters the gain of the output unit under consideration (Bonds, 1989; Heeger, 1992; Wilson \& Humanski, 1993; Carandini \& Heeger, 1994). That is, the activity level of this pool varies as the overall image content changes, and thus units tuned to different orientations are equally adjusted. However, such an adjustment might be an 'overkill' for units tuned to orientations other than orientations possessing a bias in content at a given point in time. A more ideal normalization mechanism would thus take into account the relative content biases in a scene that a given neuron is sensitive and allow for only that content to weight the output of that unit dynamically based on both the strength of that content present at a particular instant as well as in the recent past. A cortical model recently proposed by Wainwright, Schwartz, and Simoncelli (2001) is quite similar to this ideal 
but makes the dynamic weights of the filters (i.e., the modeled neural responses) a function of the likelihood of the natural scene's content that stimulates one filter given the presence of content that stimulates another filter. Specifically, the modeled neural responses are weighted by the conditional probability of image features as specified by the joint conditional histograms constructed from different filter responses to sets of natural scene imagery. That is, instead of the response of units tuned to a given orientation and spatial scale being weighted (i.e., turned down) by the relative activity of units tuned to all orientations and spatial scales, units selective for a particular orientation and spatial scale are weighted more by units tuned to similar orientations and spatial scales. Specifically, the model of Wainwright and colleagues posits that the output response is determined by each linear filter's output being half-wave rectified, squared, and then divided by a normalization signal consisting of the sum of the weighted squared responses from neighboring filters and an additive constant. The weights represent the extent to which the response of one filter is predictive of the response of the other when viewing a typical natural scene. The actual weights in their model are based on observations of conditional probabilities of simulated neural responses obtained from the statistical properties of natural signals (i.e. natural scene imagery) processed with linear filters resembling the response profile of receptive fields obtained in early visual processing areas (Simoncelli, 1999; Wainwright et al., 2001; Schwartz \& Simoncelli, 2001). Essentially the simulations were carried out with basis functions optimally 'tuned' to different spatial scales and orientations. The output responses from these different filters were successively paired in terms of all possible combinations, where, for each pair, 2D joint histograms were constructed by counting the number of response pairs 
that fell into each bin in the two-dimensional grid of a given joint histogram (see Simoncelli, Freeman, Adelson, \& Heeger, 1992; Simoncelli, 1999 for further details regarding the derivation of their basis functions and Section 3.2.1.2 for details concerning construction of the joint histograms). The typical histogram obtained with their procedure has a characteristic "bow-tie" shape (see Figure 23 for an example), suggesting that the response variance of a particular filter selective for content at one orientation, scale, and position was dependent on the given response of a 'primary' nonoverlapping filter selective for a different orientation, scale and position. This relationship was of course observed to be strongest the closer the two filters were in selectivity for orientation and scale. The primary implication here is that for a given natural scene, at any location containing 'salient' image features (i.e., prominent edges or lines), differently tuned filters will concurrently signal the presence of the same content. Thus, by using the amount of response overlap as a weighting factor to adjust the responses, Wainwright et al. (2001)'s model reduces the transmission of redundant information to successive visual processing areas. Further, this dependency is 'dynamic' in that it is completely driven by the unique structural components (i.e., image statistics) of a given natural image ${ }^{3}$. However, and most importantly, the implication of such an adjustment is that the more structural content at or near a particular orientation, the more the neural responses selective for this content will be reduced thereby effectively increasing the response thresholds at that orientation.

\footnotetext{
${ }^{3}$ This dependency was eliminated when the neural response simulation was carried out of images consisting of white noise (Schwartz \& Simoncelli, 2001; Wainwright et al., 2001).
} 
Such a model speaks directly to the content-dependent effects discussed in Sections 2.2.2.1 -2.2.2.3. For the content-dependent effect 1 described in Section 2.2.2.1, a bias in natural scene content at a given orientation and scale would drive the sensitivity of cortical units tuned to that orientation and at particular scales down more, thus accounting the psychophysical results described in that section. The content-dependent effect 2 described in Section 2.2.2.2, could be accounted for by such a model in that when the amplitude spectrum slope was very steep (increased amplitude at lower spatial scales) or very shallow (increased amplitude at higher spatial scales), units tuned to orientations in those spatial scales are more strongly activated and thus serve to carry a stronger weight in the divisive normalization pool, thereby reducing overall sensitivity in those conditions. Finally, the content-dependent effect 3 described in Section 2.2.2.3, can also be explained by such a model, working off the premise that there exist a greater number of units tuned to horizontal orientations in striate cortex. Specifically, as the amount of horizontal content increases across a series of natural scenes, the activity of units specifically tuned to horizontal orientations is reduced (a result of the content-dependent effect 1). Thus, any inhibitory effects the biased number of horizontal units had on the sensitivity of off-horizontally tuned would be released, thereby increasing their sensitivity to the oriented content in which they are tuned. However, while horizontal sensitivity in those experiments was always worst, sensitivity for detecting horizontal increments also increased with the relative amount of horizontal content. Given that response dependencies have been observed between units tuned to horizontal and units tuned to either $45^{\circ}$ and $135^{\circ}$ oblique (i.e., Wainwright et al. 2001), such an improvement 
in horizontal sensitivity can potentially by attributed to more vigorous activity of the obliquely tuned units to horizontal content. 


\section{A NEW MODEL OF NEURAL PROCESSING IN STRIATE CORTEX}

As mentioned earlier, typical normalization models propose that the output of V1 cortical units is modulated by division of their response by the summed activity of other units pooled across all orientations and spatial frequencies. However, more recent models (e.g., Wainwright et al., 2001) have dynamic components built into the divisive pool based on the probabilities of differently tuned responses co-occurring within a neural neighborhood. The results from the experiments reviewed in Sections 2.2.2.1 - 2.2.2.3 (i.e., content-dependent effects $1-3$ ) do indeed argue for a dynamic normalization of neural responses such that the weights for various orientations contributing to the normalization pool are not equal. However, such a model does not take into account the inherent horizontal effect bias found to occur in all of the experiments reviewed in the sections devoted to human visual processing of orientation in the context of broadband stimuli. Since the general horizontal effect has been demonstrated to occur with stimuli consisting of natural scenes as well as with broadband visual noise stimuli (Essock et al., 2003; Hansen \& Essock, 2003; 2004a), it appears to be due to a static anisotropy inherent in the divisive signal. Such a static component would most likely arise directly from the greater prevalence of neurons with a horizontal preferred orientation contributing more heavily to the pooled response. This numerical bias (a horizontal effect of orientation preferences) was most clearly documented by Li, Peterson, \& Freeman, (2003) recently 
in a survey of about 4,400 neurons, but is also apparent in the data of several other reports (Tiao \& Blakemore, 1976; Chapman, Stryker, \& Bonhoeffer, 1996 (easily seen in their Figures 1 and 2); Chapman \& Bonhoeffer, 1998 (easily seen in their Figures 1 and 2); Coppola, White, Fitzpatrick, \& Purves, 1998; Yu \& Shou, 2000; Mansfield, 1974; Mansfield \& Ronner, 1978). Thus an inherent weighting factor needs to be added to normalization models such that the divisive pool is influenced by both the dynamic weighting factors described earlier as well as a static anisotropic weighting factor:

$$
R_{i}=\frac{\left\lfloor L_{i}\right]^{2}}{\sum_{j}\left(\left[L_{j}^{2}\right]^{2} o_{i j} w_{i j}+\sigma_{i}^{2}\right.}
$$

The cortical model that is proposed here as possessing the most potential for accounting for the horizontal effect and the content-dependent effects was adapted from the Wainwright et al. (2001) model, where the response of linear filter $i, \mathrm{~L}_{i}$, is half-wave rectified and then squared. The result is then divided by a weighted sum of the rectified and then squared responses of the other linear filters, $\mathrm{L}_{j}$, in its respective 'neural neighborhood' weighted by the probability of these responses occurring $\left(w_{i j}\right)$ plus an error term $\left(\sigma_{i}^{2}\right)$. In this model, the $w_{i j}$ component is the weighting term that changes with respect to the strength of the different content biases in any given natural scene.

While the content-dependent effects can likely be explained in terms of the divisive normalization model posited by Wainwright et al (2001), the horizontal effect however, may stem from an innate numerical bias of neurons' preferred orientations in early cortex, which then influence the response pooling and the associated gain control 
mechanism when the pooled response to a natural scene (or other broadband content) is processed. Thus, here it is suggested that the Wainwright (2001) model be made to posses a 'inherent weighting' (i.e., $o_{i j}$ ) component that would serve to scale the nearest neighbor responses at various orientations (i.e., $\mathrm{L}_{j}$ ) according to the numerical bias of neurons tuned to different orientations. Whether this factor should be a constant factor, or made to vary with the overall amount of broadband content present in a given image is the focus of Experiments 1 and 2 of the current study and will be addressed later.

As it stands, the proposed model provides a general account for the two types of effects summarized in the preceding sections (with respect to the functions of striate simple cells - that is, the functions of the striate complex cells are not considered in such a model). However, it only shows how the different weights would be applied to the responses of striate neurons tuned to different ranges of spatial frequencies and orientations. That is, it does not show how the different weights will change as a function of the type of content bias inherent in the different types of natural scene imagery one may encounter on an everyday basis, or how the relative magnitude of inherent (or static) bias in horizontally tuned units will contribute to the general reduction of horizontal sensitivity observed in the results of all of the experiments reviewed where sensitivity to different increments of amplitude was tested. Specifically, if it is indeed the bias in the number of horizontally, and to a lesser extent vertically, tuned striate cells that causes the reduction of horizontal sensitivity to a broad spatial frequency/orientation amplitude increment, then one would expect that if the extent of the increment (in terms of total number of spatial frequencies and/or orientations incremented) is reduced, the presence 
of the horizontal effect should also diminish. Thus, it would be useful to know exactly how these weights change in the normalization pool of the proposed model as a function of stimulus content-bias as well as a function of the extent of the increment in the Fourier domain. In order to show how the inherent weights (i.e., $o_{i j}$ ) might change as a function of increment extent (that is, extent in the Fourier domain), a series of psychophysical experiments need to be carried out. In addition, in order to show how the dynamic weights (i.e., $w_{i j}$ ) change as a function of content bias contained in natural scene stimuli, a protocol for carrying out simulated neural responses with the natural scene stimuli used in the experiments that demonstrated the content-dependent effects $1-3$ should also be carried out. Thus, the current study sought out to carry out experiments in order to demonstrate how the inherent and dynamic weights of the proposed model would change as a function of amplitude increment extent and amount of phase defined structural content of natural scenes, respectively. However, in the interest of practicality, the changes in both of the types of weights will be described separately for the two effects. First, a set of psychophysical experiments were employed in onder demonstrate how the inherent orientation performance bias changes as a function of amplitude increment extent (as well as show how the inherent weights, $o_{i j}$, change as a function of increment extent). Secondly, several different simulated neural response paradigms were also carried out in order to demonstrate how the dynamic weights, $w_{i j}$, change as a function of the type of content bias present in the different sets of natural scene imagery described earlier. 


\section{GENERAL METHODS}

\subsection{Psychophysical Experiments}

As mentioned in the preceding section, should the horizontal effect (i.e., the inherent or static effect) arise out of a cortical gain mechanism which operates to reduce sensitivity to broadband horizontal increments (and to a lesser extent, broadband vertical increments) through the pooling of those cortical units, then one might predict that if the extent of the amplitude increments is systematically limited, then the horizontal effect would be abolished. The reasoning here is that since the extent of the increments in the Fourier domain spanned across all possible spatial frequencies and contained a range of orientations ( $45^{\circ}$ bandwidth centered on one of the nominal orientations), a large portion of the biased number of horizontally tuned cortical units (and to a lesser extent, vertically tuned units) are summed in the normalization pool which would in turn lead to larger reductions in sensitivity to those orientations. However, if the extent of the increment in the Fourier domain is made to contain only a small range of spatial frequencies and orientations, then the total number of horizontally and vertically tuned cells summed in the normalization pool would be smaller and the decrease in the sensitivity to those orientations would not be as great. In order to test such a hypothesis, a series of psychophysical experiments where the extent of the orientation increments in the Fourier domain is systematically reduced, were carried out. The data from these experiments show how the inherent weights of the proposed model would change as more and more 
striate neuron responses are summed (i.e., in response to the extent of amplitude increments). The results from the psychophysical experiments serve two primary purposes: 1) provide support for the idea that the horizontal effect (i.e., inherent orientation processing bias) arises out of an anisotropic gain adjustment, and 2) provide the necessary data allowing for a functional description of orientation processing in broadband stimuli.

Two 16-condition experiments (repeated measures design) were carried out. The psychophysical paradigm of the experiments consisted of a suprathreshold matching procedure (i.e., method of adjustment) similar to that described in Section 2.2.1. The first experiment, Experiment 1, was designed to better understand how human visual processing of orientation in the context of naturalistic broadband stimuli changes as a function of the extent of the amplitude increment. Briefly, the conditions of this experiment involved systematically limiting the extent of the broad spatial frequency, broad orientation amplitude increment to a single point in the Fourier domain applied to amplitude spectra with an $\alpha$ value equal to 1.0 . That is, the spatial stimuli consisted of $1 / f$ visual noise patterns that were made to possess amplitude increments of varying extent at one of the four nominal orientations (i.e., increments presented on a background of $1 / f$ noise). The fundamental idea behind Experiment 1 was to examine how participants perceived specific orientations against a background of $1 / f$ noise. Such a background will presumably activate all striate neurons tuned to all orientations and spatial frequencies (activation similar to that which would occur when viewing natural scenes). Thus, the design of Experiment 1 allowed for the examination of how a range of striate 
neurons tuned to a range of spatial frequencies and orientations function in a population of activated neurons (activated by the $1 / f$ noise background) tuned to other orientations and spatial frequencies.

The second experiment, Experiment 2, was set up in an identical manner as Experiment 1, except after the increments are applied to the $1 / f$ amplitude spectra, the portions of the spectra that were not been incremented were be filtered out. Thus the spatial stimuli only consisted of the content contained within the extent of the increment (i.e., increments presented without the $1 / f$ noise background). Experiment 2 was designed to allow for a better understanding of how sensitivity to a given oriented stimulus, for example, a sine wave grating, changes as more and more sine waves of similar spatial frequencies and orientations are added to it. Thus, the saliency of different ranges of spatial frequencies and orientations could be examined in the absence of any interactions from the activity of other differently tuned cortical units due to the presence of a broadband $1 / f$ noise background. Specifically, the results from Experiment 2 provide insight into how the inherent weights of the proposed striate normalization model changed for different amplitude increment extents without any influence from a $1 / f$ background. The fundamental purpose for carrying out these two experiments (variable extent increments with and without a broadband $1 /$ f background) was so that the results could be compared in order to determine with a fair amount of accuracy what portion of the inherent weights (i.e., $o_{i j}$ ) are strictly due to the pooled activity of similarly tuned cortical units (Experiment 2 - the 'increment-alone' experiment) and what portion of those weights can be attributed to the interactions from other differently tuned cells 
responding to the $1 / f$ noise background (Experiment 1 - the 'increment-on-background' experiment).

Thus in both of the psychophysical experiments, suprathreshold sensitivity for different increment extents ranging from a single orientation (set at one of the four nominal orientations of course) and spatial frequency (i.e., $16 \mathrm{cpd}$ ) to the extent utilized by Essock et al. (2003) (i.e., broadband spatial frequency and $45^{\circ}$ orientation bandwidth). will be examined with and without a $1 / f$ noise background. The fundamental reason behind testing such a large range of filter extents was to examine suprathreshold sensitivity to a high spatial frequency sinusoidal grating embedded in noise, which should, if the proposed striate normalization model for striate visual processing is accurate, produce a different pattern of results (ideally, some form of an oblique effect) compared to the pattern of results already shown to occur for broad frequency, $45^{\circ}$ orientation bandwidth triangle filter extents (i.e., a horizontal effect. Thus under such a paradigm, the ideal set of results would yield some form of oblique effect when the triangle filter is highly limiting with respect to the number of spatial frequencies and orientations incremented and as more and more orientations and spatial frequencies are incremented, would gradually (or rapidly) shift toward a horizontal effect. Also, the current experimental paradigm would allow one to determine if, while holding either spatial frequency or orientation constant, adding more and more spatial frequencies or more and more orientations caused the switch from an oblique effect to a horizontal effect to be more rapid. That is, is it the effect of adding more orientations at any given range of spatial frequencies yield a shift over to a horizontal effect more rapidly than 
when more spatial frequencies are added to a given range of orientations included in the filter extent or vice versa. Since the above examinations was carried out with triangle filter increments in the presence of a noise background or alone also allowed for the determination of the effects a broadband noise background had on the saliency of different ranges of spatial frequency and orientation increments.

The planned comparisons that will be carried out include examining the interactions of the different patterns of results for each condition as more and more spatial frequencies were incremented, or as more and more orientations were incremented. Additionally, these interactions will be examined in the context of whether or not the different increment extents were matched in the presence of a broadband noise background (i.e., Experiment 1) or presented alone (i.e., Experiment 2). In order of importance, the planned comparisons are as follows. 1) Between the two experiments, a three-way repeated measures Analysis of Variance (ANOVA) will be carried out between the data obtained from each of the 16 conditions of both Experiments 1 and 2 in order to determine whether or not the presence/absence of a broadband noise pattern changed the overall pattern of results observed in each of the 16 conditions for both experiments. Thus, the three-way interaction (background type-by-increment extent-by-orientation) will be examined. 2) Within each experiment, the overall effect of triangle filter extent on the perceptual matches of the four orientations will be examined with a two-way repeated measures ANOVA to determine whether or not a significant interaction between the 16 different conditions is present. 3) Within each experiment, the results from the 16 different conditions will be subjected to two-way repeated measures ANOVAs by 
grouping the data with respect to $a$ ) each of the four different spatial frequency filter extents where orientation was allowed to vary. The reason for that comparison will be to determine if the pattern of results in those conditions interacted significantly as a function of increasing numbers of incremented orientations. b) The conditions will be grouped with respect to the four different orientation bandwidth filter extents where spatial frequency was allowed to vary. The reason for that comparison will be to determine if the pattern of results in those conditions interacted significantly as a function of increasing numbers of incremented spatial frequencies. 4) Also of importance is the comparison of the data as grouped by $3 a$ and $3 b$ between the background and nobackground experiments in order to determine whether or not the interaction across the rows or columns (i.e., the groups of data as described in parts $3 a$ and $3 b$ stated above) in the Experiment 1 interacts significantly with the same grouped data from Experiment 2. The fundamental reason for examining this relationship is to determine if the change in the pattern of results for the four conditions of each group (i.e., change from an oblique effect pattern to a horizontal effect pattern) differed as a function of background type (i.e., broadband noise present or absent). Such an analysis is important in determining whether or not the presence/absence of the broadband background affected the predicted shift from an oblique effect in the conditions exhibiting an oblique effect pattern of results to the conditions exhibiting a horizontal effect pattern of results. 5) Lastly, and of minor importance, the data obtained from each of the conditions within each experiment will be subjected to one-way repeated measures ANOVAs in order to determine if the perceptual matches for the four different orientations within each condition differed significantly. 


\subsubsection{Experiment 1}

\section{Apparatus}

Standard stimuli were presented on a 21" Sony Trinitron monitor that was mounted to a platform which allowed the monitor to be rotated and fixed $22.5^{\circ}$ rightward of vertical (refer to the Stimuli Generation section of Experiment 1 for an explanation for why rotation of this monitor was necessary). The test stimuli were presented on a 21" SGI $420 \mathrm{C}$ monitor. The distance between the centers of the two monitors was $60 \mathrm{~mm}\left(13.2^{\circ}\right.$ visual angle). To eliminate edge contours from the room and monitor bezels, a circular mask subtending $27^{\circ}$ visual angle with two circular stimulus apertures, each subtending $5.8^{\circ}$ visual angle (centered around both the standard and test patterns) was fit to the monitors. Resolution for both monitors was set at $800 \times 600$, the frame rate of the Sony Trinitron monitor was $100 \mathrm{~Hz}$, and for the SGI $420 \mathrm{C}$ monitor, the frame rate was $120 \mathrm{~Hz}$. Both monitors had a maximum luminance of $80 \mathrm{~cd} / \mathrm{m}^{2}$, and were calibrated with a IL1700 Research Radiometer to have a linear output. The height from the central portion of each monitor to the floor was at eye level of the seated observer. Both monitors were driven by a Dell Pentium IV PC (2.61 Ghz processor) with a dual monitor card which was run off of nVidia graphics software to update both monitors. During the experiments, the lights were turned off, thus eliminating any effects due to external room contours. A chin-and-forehead rest was utilized in order to eliminate any head movements. Participants were seated $2.57 \mathrm{~m}$ from the displays, and were aligned with the center of the circular mask. 


\section{Participants}

Nine participants were recruited for both experiments ( 7 of which were naïve to the purpose of the experiments). All participants had normal or corrected to normal vision. For those with corrected vision, a series of vision tests were carried out to assure that they did not have any residual astigmatism. The age range of the participants was $22-35$ and IRB-approved informed consent was obtained. In order to familiarize the participants with the experiments, practice sessions were allowed. All naïve participants were paid $\$ 10$ per session.

\subsubsection{Stimulus Generation for Experiment 1}

The software platform used to create all of the stimuli was MATLAB version 6.5with accompanying Image and Signal Processing toolboxes (versions 4.0 and 6.1 respectively). The stimuli for the current experiment consisted of $512 \times 512$ pixel, broadband visual noise patterns. These patterns were constructed in the Fourier domain by combining a random phase matrix (created by randomly assigning values in the range of $-\pi$ to $\pi$ to a $512 \times 512$ matrix) and an isotropic amplitude spectrum of appropriate dimensions with an a value equal to 1.0. The reasoning behind using visual noise patterns is the same as that mentioned in Section 2.2.1. For practical reasons, only five noise patterns were used, and in their un-altered state possessed the same mean (grayscale value 128 ) and r.m.s. contrast (i.e., $\mathrm{SD} \approx 44$ ) in the spatial domain. The filter used for making the increments to the amplitude spectrum consisted of the triangular 'wedge' also mentioned in Section 2.2.1. The primary reason for selecting the triangle wedge filter to 
make the increments at the nominal orientations was based on the fact that, for natural scenes, the bias in amplitude at any given orientation is triangular in nature. Specifically, when a bias in amplitude is found for a given image, the amplitude spectrum of that scene will exhibit a peak centered on an orientation vector that ramps down across adjacent orientations. An example of such a bias is shown in Figure 24 which depicts an averaged spectrum from 88 natural scene images that contained a bias in amplitude at horizontal orientations (separate analyses for images containing amplitude biases at other orientations reveal a similar patterns). Therefore, using the triangle filter to increment the isotropic spectra of the noise patterns would create amplitude biases that most closely resemble the form of the bias of the amplitude spectra of typical natural scenes.

The magnitude of the triangle increments used in Experment 1 were determined by selecting a scalar value greater than one (e.g., 1.5). Applying the scalar value to the triangle filter resulted in a triangular function with a peak equal to the value of the scalar which was linearly ramped down to 1.0 in polar coordinates across the selected orientation bandwidth of the triangle filter for all spatial frequencies below the Nyquist limit (no increment was applied to the DC component of the spectrum). Thus, for the current experiment, the extent of the increment refers to the number of spatial frequencies included in the filter as well as its orientation bandwidth which was centered on one of four orientations $\left(0^{\circ}, 45^{\circ}, 90^{\circ}\right.$, or $\left.135^{\circ}\right)$. Orientation was referenced in the Fourier domain, thus $0^{\circ}$ refers to a vertical increment in the spatial domain. In the spatial domain (after image generation and application of the increment), all patterns were fit with a circular 'edge-blurred' window which ramped the stimulus pixel values down to the 
mean (i.e., 128 at $40 \mathrm{~cd} / \mathrm{m}^{2}$ ). The above methods for stimulus generation are exactly those that were implemented in previously published studies by Essock et al (2003) and Hansen and Essock (2004a), refer to Figure 25 for further details of the filtering process used to create the stimuli for the current experiment.

For the 16 conditions in the current experiment, the triangle filter was systematically limited with respect to number of total spatial frequencies and orientations that could be incremented. Figure 26 depicts examples of the different increment extents (for one orientation) for each of the 16 conditions of Experiment 1; specifically, each cell is an experimental condition (again, the increment was centered on one of the four orientations mentioned earlier). Because of the high likelihood of sampling error at the lower spatial frequency range of the amplitude spectrum (refer to Section 1.2), in the conditions where spatial frequency is the limiting factor, only the higher spatial frequencies were examined. One benefit of this approach is that it allowed for the current experiment to determine if human participants show an oblique effect for the conditions where the increment was applied to the higher spatial frequencies only. For each orientation in a given condition, the magnitude of the increment was varied in equal step sizes, with the scalar values ranging from 1.0 (no increment) to a scalar value that yields no more than $\sim 30 \%$ saturation on the pixel values in the spatial domain; because of the different extents of the increment filter, this range was allowed to vary. Thus for each of the noise patterns in each of the 16 conditions, sets of noise patterns will be generated prior to the experiments consisting of an extensive range of increment sizes for each of the four nominal orientations. Table 1 makes explicit the spatial frequency and orientations in 
which the triangle increment filter was limited for the 16 different conditions of Experiment 1.

\subsubsection{Psychophysical Paradigm for Experiment 1}

The psychophysical paradigm for Experiment 1 consisted of a suprathreshold matching procedure (method of adjustment; e.g. Falmagne, 1986) which followed very closely the methods utilized by Essock et al. (2003). Briefly, this procedure involved presenting two laterally displaced patterns. On the left was a standard stimulus pattern possessing a fixed increment magnitude at either $22.5^{\circ}$ or $112.5^{\circ}$; on the right was a test pattern possessing a variable increment magnitude at one of the four nominal orientations (where the initial increment magnitude will be determined randomly) presented randomly. Observers were allowed to adjust the magnitude of the increment present in the test pattern in order to make a perceptual match to the amount of oriented content present in the standard pattern via key-press (see Figure 27). For all conditions, the same five noise patterns were used, but the phase of the standard and test patterns were never the same. Observers were instructed to match the amount of oriented content in the test pattern in order to make a perceptual match to amount of oriented content present in the standard pattern regardless of orientation. In addition, participants were told to match the oriented content with respect to the entire pattern and not to local areas. Within one condition, observers made 40 matches ( 10 per nominal orientation, 5 per standard orientation). Each condition was repeated four times with all conditions randomized (totaling 160 perceptual matches per condition), with four conditions being carried out per day. All of which required a total of 16 days to complete (not counting practice trials). 
Regarding the orientation of the fixed increment of the standard patterns, due to the sampling errors incurred at the lower spatial frequencies mentioned in Section 1.2, a full broadband increment (i.e., all spatial frequencies allowed by the Nyquist limit within a given orientation bandwidth) could not be generated in the Fourier domain for all of the conditions. In order to address this issue, the monitor presenting the standard stimulus was rotated $22.5^{\circ}$ right of vertical, thus vertical and horizontal incremented patterns were presented. Given that there was no practical way to determine the optimal fixed orientation increment value of the standard patterns, the pattern in the center of the range of increments for each condition was used as the standard increment. Again, this methodology follows from the methods employed by Essock et al. (2003).

\subsubsection{Experiment 2}

\section{Apparatus}

All equipment and respective parameter setting were identical to those utilized in Experiment 1.

Participants

The same participants that were in Experiment 1 were also in the current experiment. 


\subsubsection{Stimulus Generation for Experiment 2}

The software platform used to create all of the stimuli was MATLAB version 6.5with accompanying Image and Signal Processing toolboxes (versions 4.0 and 6.1 respectively). The stimuli for the current experiment consisted of $512 \times 512$ pixel, broadband visual noise patterns. These patterns were constructed in the Fourier domain by combining a random phase matrix (created by randomly assigning values in the range of $-\pi$ to $\pi$ to a $512 \times 512$ matrix) and an isotropic amplitude spectrum of appropriate dimensions with an $\alpha$ value equal to 1.0. The reasoning behind using visual noise patterns was the same as that mentioned in Section 2.2.1. For practical reasons, only five noise patterns were used, and in their un-altered state will have the same mean (grayscale value 128 ) and r.m.s. contrast (i.e., $\mathrm{SD} \approx 44$ ) in the spatial domain; and were identical to those used in Experiment 1. The filter used for making the increments to the amplitude spectrum consisted of the same triangular 'wedge' utilized in Experiment 1. The magnitude and extent of the increment itself was determined by the same methods discussed in Experiment 1. As in Experiment 1, orientation is referenced in the Fourier domain, thus $0^{\circ}$ refers to a vertical increment in the spatial domain. In the spatial domain (after image generation and application of the increment), all patterns were fit with a circular 'edge-blurred' window which ramped the stimulus pixel values down to the mean (i.e., 128 at $40 \mathrm{~cd} / \mathrm{m}^{2}$ ). For the 16 conditions of the current experiment, the triangle filter was systematically limited with respect to number of total spatial frequencies and orientations that were incremented as in Experiment 1, only here, the background was set to zeros so that only the content falling under the increment was allowed to pass. Figure 28 depicts an example of the filtering process, refer to Table 1 for an explicit description 
of the triangle filter extents for the different conditions of the current experiment. Again, because of the high likelihood of sampling error at the lower spatial frequency range of the amplitude spectrum (refer to Section 1.2), in the conditions where spatial frequency is the limiting factor, only the higher spatial frequencies were examined. For each orientation in a given condition, the magnitude of the increment was varied in equal step sizes, with the scalar values ranging from 1.0 (no increment) to a scalar value that yields no more than $\sim 30 \%$ saturation on the pixel values in the spatial domain; because of the different extents of the increment filter, this range was allowed to vary. Thus for each of the 'no-background' patterns in each of the 16 conditions, sets of patterns were generated prior to the experiments consisting of an extensive range of increment sizes for each of the four nominal orientations.

\subsubsection{Psychophysical Paradigm for Experiment 2}

The paradigm for Experiment 2 is identical to that of Experiment 1, with the one difference being the patterns consisted increments made to specific ranges of oriented content in the absence of a broadband $1 / f$ background. The instructions given to each of the participants were to match the strength of the oriented content in the test pattern to match that of the standard pattern without regard to orientation and to match the global pattern (i.e., they were asked not to make perceptual matches based on the local features of the patterns). 


\section{PSYCHOPHYSICAL EXPERIMENT RESULTS}

For both Experiment 1 and 2, participants made perceptual matches between the amount of oriented content/structure contained in a given test pattern relative to a given standard pattern (with the amount of oriented content in the standard pattern held constant).

Physically however, participants were actually matching the magnitude of the amplitude increment in the test pattern to that of the standard pattern. Since scalar values were used to generate the amplitude increments of varying magnitude, when a participant selected a given test pattern as a perceptual match, the scalar value used to scale the amplitude increment for that pattern was written to the data file. Within each session for any given condition, participants made ten perceptual matches for each orientation ( 5 matches to the $22.5^{\circ}$ standard pattern and 5 matches to the $112.5^{\circ}$ standard pattern). Thus data from each condition and for each of the four orientations were assembled by taking the mean and standard error across the two standard matching patterns.

The analysis of the data obtained from both Experiment 1 and 2 began by first transforming the scalar means into ratios (test increment scalar to standard increment scalar). The primary reason for taking the test to standard ratio was based on the fact that the magnitudes of the increments of the different conditions were often very different. What the ratios indicate about the different perceptual matches is relatively straight forward in that values above 1.0 indicate that the incremented content in the test pattern 
was less salient relative to the standard pattern and values below 1.0 indicate that the incremented content in the test pattern was more salient relative to the standard pattern.

\subsection{Experiment 1 Results}

The results from the 16 different suprathreshold matching conditions of Experiment 1 are plotted in Figure 29 and are averaged across all of the nine participants (the results for individual observers are plotted in the Appendix). The spatial layout of this figure follows that laid out in Figure 26 and Table 1 with respect to increment extent. For example, the bottom left graph plots the average test increment scalar to standard increment scalar ratio for the single orientation/single spatial frequency increment extent; the top right graph plots the data from the broadband spatial frequency, $45^{\circ}$ orientation bandwidth increment. Notice that each row of this figure holds a given spatial frequency range constant (starting with the single spatial frequency increment extent on the bottom to the broadband spatial frequency increment extent on the top). As one moves across each cell for any given row, the number of orientations in the increment varies from a single orientation (far left) to the $45^{\circ}$ orientation bandwidth increment extent (far right). Similarly, each cell within a given column holds a particular orientation bandwidth constant and the cells in each column plot data from conditions where spatial frequency extent of the increment varied (i.e., single spatial frequency increment extent in the bottom cells and, as one move up a given column, increases to the broadband spatial frequency increment extent in the top cells). On the ordinate of each graph is the averaged test-to-standard increment magnitude (i.e., the ratio of the test and 
standard scalar values) and on the abscissa are the four different orientations in which the participants made perceptual matches.

Before discussing the statistical analyses, it should be noted that spatial layout of Figure 29 as well as Table 1 will be referred to often in the current section in order to describe how the different conditions were grouped for some of the different analyses described below. As mentioned toward the end of Section 5.1, there were five primary sets of planned comparisons that would be carried out on the data obtained from Experiment 1 and 2; three of which were applied to the data obtained from each individual experiment.

\subsubsection{Planned Comparisons: The Interaction Across All Conditions}

The first analysis that was carried out was designed to test whether or not there was a significant interaction among the pattern of results obtained from each of the 16 conditions of the current experiment. In order to examine that interaction, a $16 \times 4$ (16 different increment extents by 4 different orientations) two-way repeated measures Analysis of Variance (ANOVA) was carried out. As Figure 29 suggests, this interaction was indeed significant $\left(\mathrm{F}_{(45,360)}=7.84, \mathrm{p}<.001\right)$ indicating significant differences in the pattern of results between the 16 conditions of the current experiment.

\subsubsection{Planned Comparisons: Interactions Within Spatial Frequency/Orientation Bands}

The next analysis involved grouping the data from the 16 different conditions with respect to 1) each of the four different spatial frequency filter extents where orientation 
was allowed to vary and 2) with respect to the four different orientation bandwidth filter extents where spatial frequency was allowed to vary. Using Figure 29 as a reference, the two analyses mentioned above will be looking at whether or not there is a significant interaction across the four conditions within each of the four rows (spatial frequency held constant within each row) and across the four conditions in each of the four columns (orientation bandwidth held constant within each column). Each row or column was subjected to a $4 \times 4$ (variable spatial frequency or orientation bandwidth by test orientation) two-way repeated measures ANOVA in order to examine the interaction across conditions for each row or column in Figure 29.

\subsubsection{Planned Comparisons: Interactions Within Spatial Frequency Bands}

The analysis for the four conditions where the spatial frequency of the increment was held at $16 \mathrm{cpd}$, and orientation bandwidth was allowed to vary (e.g., single orientation, $5^{\circ}$ orientation bandwidth, $20^{\circ}$ orientation bandwidth, and $45^{\circ}$ orientation bandwidth) yielded a significant interaction $\left(\mathrm{F}_{(9,72)}=6.11, \mathrm{p}<.001\right)$. The interaction for the four conditions where the spatial frequency of the increment was held at a $1 / 2$ octave $(12-16 \mathrm{cpd})$, with variable orientation bandwidths was significant $\left(\mathrm{F}_{(9,72)}=2.31, \mathrm{p}=.02\right)$. The interaction for the four conditions where spatial frequency of the increment was held at 1 octave (8 $16 \mathrm{cpd})$, with variable orientation bandwidths was significant $\left(\mathrm{F}_{(9,72)}=25.39, \mathrm{p}<.001\right)$. Lastly, the interaction for the four conditions where spatial frequency of the increment was broadband ( $0.2-16 \mathrm{cpd})$, with variable orientation bandwidths was significant $\left(\mathrm{F}_{(9}\right.$, 72) $=18.85, \mathrm{p}<.001)$ 


\subsubsection{Planned Comparisons: Interactions Within Orientation Bands}

The analysis for the four conditions where the orientation bandwidth of the increment was held at a single orientation, and spatial frequency bandwidth was allowed to vary (e.g., $16 \mathrm{cpd}, 12-16 \mathrm{cpd}, 8-16 \mathrm{cpd}$, and $0.2-16 \mathrm{cpd}$ ) yielded a significant interaction $\left(F_{(9,72)}=3.10, p=.003\right)$. The interaction for the four conditions where the orientation bandwidth of the increment was held at $5^{\circ}$, with variable spatial frequency bandwidths was not significant $\left(\mathrm{F}_{(9,72)}=1.97, \mathrm{p}=.056\right)$. The interaction for the four conditions where the orientation bandwidth of the increment was held at $20^{\circ}$, with variable spatial frequency bandwidths was significant $\left(\mathrm{F}_{(9,72)}=4.04, \mathrm{p}<.001\right)$. Lastly, the interaction for the four conditions where the orientation bandwidth of the increment was held at $45^{\circ}$, with variable spatial frequency bandwidths was significant $\left(\mathrm{F}_{(9,72)}=10.31, \mathrm{p}<.001\right)$.

\subsubsection{Planned Comparisons: Interactions Within Each Condition}

Since the overall interaction between all conditions of the current experiment was significant, the final set of statistical analyses involved examining whether or not the differences between the four orientations within each condition were significant. Thus, the data from each condition were subjected to a one-way repeated measures ANOVA. The significant effects of orientation were found to occur in the conditions contained in the first column of Figure 29 (i.e., Conditions 1, 5, 9, and 13 described in Table 1), and in three of the four conditions in the top row of Figure 29 (i.e., Conditions 13, 15, and 10). For the significant conditions contained in the first column, the F-ratios were as follows: Condition 1: $\mathrm{F}_{(3,24)}=6.83, \mathrm{p}=.002$; Condition 5: $\mathrm{F}_{(3,24)}=6.14, \mathrm{p}=.003$; Condition 9: $F_{(3,24)}=22.15, p<.001 ;$ and Condition 13: $F_{(3,24)}=6.12, p=.003$. For the 
significant conditions contained in the top row not already reported, the F-ratios were as follows: Condition 15: $\mathrm{F}_{(3,24)}=4.0, \mathrm{p}=.02$ and Condition 16: $\mathrm{F}_{(3,24)}=15.90, \mathrm{p}<.001$. All other conditions did not yield significant effects of orientation at the $p<.05$ level.

\subsubsection{General Summary of the Results from Experiment 1}

Again, referring to the spatial layout of Figure 29, the significant interaction across all conditions of the current experiment suggests that the pattern of perceptual matches made for each of the four orientations in each condition changed as a result of differing triangle filter increment extents. What is clear in Figure 29 is that the conditions with the most limiting triangle filter extents (i.e., the bottom row and the left-most column), an oblique effect pattern of results can be seen in most of those graphs. However, both yielded significant interactions, suggesting differences in the 'types' of oblique effect patterns across that row and that column. However, the significant interaction across the bottom row was most likely caused by Condition 2 (see Table 1) since that condition did not yield any particular orientation effect, in fact, when that condition was left out of the analysis, the interaction across that row was not significant $\left(\mathrm{F}_{(6,48)}=2.3, \mathrm{p}=.10\right)$. With respect to the significant interaction for the left-most column, while each graph shows an oblique effect pattern of results, the sheer magnitude of the oblique effect pattern in Condition 9 likely interacted with the other three conditions. The latter supposition was supported when that condition was left out of the analysis in that the interaction across the left-most column was no longer significant $\left(\mathrm{F}_{(6,48)}=.791, \mathrm{p}=.582\right)$. With the exception of one column (see Section 6.1.2.2), the other columns and rows all yielded significant interactions, indicating that their were significant changes in the pattern of 
perceptual matches (shifting from an oblique effect pattern of results to a horizontal effect pattern of results) in conditions where the triangle filter extents ranged from intermediate to large. Finally, while the conditions in the left-most column and bottom row exhibited varying degrees of oblique effect patterns, only those in the conditions in the left-most column produced significant effects of orientation. Likewise, while the upper-right quadrant of Figure 29 contains the conditions where the horizontal effect is most evident, only Conditions 15 and 16 produced significant effects of orientation.

\subsection{Experiment 2 Results}

The results from the 16 different suprathreshold matching conditions of Experiment 2 are plotted in Figure $\mathbf{3 0}$ and are averaged across all of the nine participants (the results for individual observers are plotted in the Appendix). The spatial layout of this figure follows that laid out in Figure 29 and Table 1 with respect to increment extent (refer to Section 6.1 for further details regarding the spatial layout of this figure). As in the results section of Experiment 1, the spatial layout of Figure 29 as well as Table 1 will be referred to often in the current section in order to describe how the different conditions were grouped for some of the different analyses described below. The planned comparisons for the current data will be identical to those carried out in the results section of Experiment 1 and are presented in the following sections.

\subsubsection{Planned Comparisons: The Interaction Across All Conditions}

The first analysis that was carried out was designed to test whether or not there was a significant interaction among the pattern of results obtained from each of the 16 
conditions of the current experiment. In order to examine that interaction, a $16 \times 4$ (16 different increment extents by 4 different orientations) two-way repeated measures Analysis of Variance (ANOVA) was carried out. As Figure 30 suggests, this interaction was indeed significant $\left(\mathrm{F}_{(45,360)}=5.51, \mathrm{p}<.001\right)$ indicating significant differences in the pattern of results between the 16 conditions of the current experiment.

\subsubsection{Planned Comparisons: Interactions Within Spatial Frequency/Orientation Bands} The next analysis involved grouping the data from the 16 different conditions with respect to 1) each of the four different spatial frequency filter extents where orientation was allowed to vary and 2) with respect to the four different orientation bandwidth filter extents where spatial frequency was allowed to vary. Using Figure $\mathbf{3 0}$ as a reference, the two analyses mentioned above will be looking at whether or not there is a significant interaction across the four conditions within each of the four rows (spatial frequency held constant within each row) and across the four conditions in each of the four columns (orientation bandwidth held constant within each column). Each row or column was subjected to a $4 \times 4$ (variable spatial frequency or orientation bandwidth by test orientation) two-way repeated measures ANOVA in order to examine the interaction across conditions for each row or column in Figure 30.

\subsubsection{Planned Comparisons: Interactions Within Spatial Frequency Bands}

The analysis for the four conditions where the spatial frequency of the increment was held at $16 \mathrm{cpd}$, and orientation bandwidth was allowed to vary (e.g., single orientation, $5^{\circ}$ orientation bandwidth, $20^{\circ}$ orientation bandwidth, and $45^{\circ}$ orientation bandwidth) did not 
yield a significant interaction $\left(F_{(9,72)}=1.06, p=.401\right)$. The interaction for the four conditions where the spatial frequency of the increment was held at a $1 / 2$ octave (12 $16 \mathrm{cpd})$, with variable orientation bandwidths was significant $\left(\mathrm{F}_{(9,72)}=4.04, \mathrm{p}<.001\right)$. The interaction for the four conditions where spatial frequency of the increment was held at 1 octave $(8-16 \mathrm{cpd})$, with variable orientation bandwidths was significant $\left(\mathrm{F}_{(9,72)}=\right.$ $14.39, \mathrm{p}<.001)$. Lastly, the interaction for the four conditions where spatial frequency of the increment was broadband $(0.2-16 \mathrm{cpd})$, with variable orientation bandwidths was significant $\left(\mathrm{F}_{(9,72)}=8.74, \mathrm{p}<.001\right)$.

\subsubsection{Planned Comparisons: Interactions Within Orientation Bands}

The analysis for the four conditions where the orientation bandwidth of the increment was held at a single orientation, and spatial frequency bandwidth was allowed to vary (e.g., $16 \mathrm{cpd}, 12-16 \mathrm{cpd}, 8-16 \mathrm{cpd}$, and $0.2-16 \mathrm{cpd}$ ) yielded a significant interaction $\left(F_{(9,72)}=2.78, p=.007\right)$. The interaction for the four conditions where the orientation bandwidth of the increment was held at $5^{\circ}$, with variable spatial frequency bandwidths was significant $\left(F_{(9,72)}=7.80, p<.001\right)$. The interaction for the four conditions where the orientation bandwidth of the increment was held at $20^{\circ}$, with variable spatial frequency bandwidths was significant $\left(\mathrm{F}_{(9,72)}=4.57, \mathrm{p}<.001\right)$. Lastly, the interaction for the four conditions where the orientation bandwidth of the increment was held at $45^{\circ}$, with variable spatial frequency bandwidths was significant $\left(\mathrm{F}_{(9,72)}=5.28, \mathrm{p}<.001\right)$. 


\subsubsection{Planned Comparisons: Interactions Within Each Condition}

Since the overall interaction between all conditions of the current experiment was significant, the final set of statistical analyses involved examining whether or not the differences between the four orientations within each condition were significant. Thus, the data from each condition were subjected to a one-way repeated measures ANOVA. The significant effects of orientation were found to occur in three of the four conditions contained in the first column of Figure 30 (i.e., Conditions 5,9 , and 13 described in Table 1), and in all of the conditions in the top row of Figure 30 (i.e., Conditions 13, 14, 15, and 16). For the significant conditions contained in the first column, the F-ratios were as follows: Condition 5: $F_{(3,24)}=5.78, p=.004 ;$ Condition 9: $F_{(3,24)}=7.12, p=$ .001 ; and Condition 13: $\mathrm{F}_{(3,24)}=13.53, \mathrm{p}<.001$. For the significant conditions contained in the top row not already reported, the F-ratios were as follows: Condition 14: $\mathrm{F}_{(3,24)}=$ 9.27, $\mathrm{p}<.001 ;$ Condition 15: $\mathrm{F}_{(3,24)}=6.18, \mathrm{p}=.003$ and Condition $16: \mathrm{F}_{(3,24)}=5.23, \mathrm{p}=$ .006. All other conditions did not yield significant effects of orientation at the $p<.05$ level.

\subsubsection{General Summary of the Results from Experiment 2}

Again, referring to the spatial layout of Figure 30, the significant interaction across all conditions of the current experiment suggests that the pattern of perceptual matches made for each of the four orientations in each condition changed as a result of differing triangle filter increment extents. Overall, the planned comparisons of the data obtained in Experiment 2 yielded results very similar to those obtained form Experiment 1 where the amplitude increments were embedded in broadband $1 / f$ noise. Additionally, the 
conditions where the triangle filter increment extent was most limited (i.e., the left-most column and bottom row), in general, yielded oblique effect patterns with a few minor exceptions. For example, as in the left-most column of the graph matrix plotted in Figure 29, the left-most column of the graph matrix plotted in Figure 30, in general, shows oblique effect patterns with the exception of Condition 13 where the pattern is there, but the horizontal matches were much more elevated than the other three conditions in that column. Thus, it is very likely that Condition 13 is the underlying cause of the significant interaction obtained from the $4 \times 4$ two-way repeated measures analysis for the conditions in the left-most column. Accordingly, when that condition is left out of the analysis, the interaction is no longer significant $\left(\mathrm{F}_{(6,48)}=1.09, \mathrm{p}=.377\right)$. Unlike the results of the statistical analysis of the data contained in the bottom row from Figure 29 (i.e., Experiment 1), the interaction for that row from the current experiment resulted in a non-significant interaction. The indication here is two-fold, either the oblique effect patterns in those graphs did not differ by much, or the data from the individual observers was simply too noisy to yield significant results. By examining the standard error bars (which are quite large in those graphs) in those graphs, the latter explanation appears to be the most likely. With the exception of one row (see Section 6.2.2.2), the other columns and rows all yielded significant interactions, indicating that their were significant changes in the pattern of perceptual matches (shifting from an oblique effect pattern of results to a horizontal effect pattern of results) in conditions where the triangle filter extents ranged from intermediate to large. Finally, while the conditions in the leftmost column and bottom row exhibited varying degrees of oblique effect patterns, only those in the conditions in the left-most column produced significant effects of orientation. 
Likewise, while the upper-right quadrant of Figure 30 contains the conditions where the horizontal effect is most evident, only Conditions 15 and 16 produced significant effects of orientation.

\subsection{The Interactions Between Experiment 1 and Experiment 2}

The results from the 16 different suprathreshold matching conditions of Experiments 1 and 2 are plotted in Figure 31 and are averaged across all of the nine participants. Thus, in each graph is plotted the averaged data from the nine participants from each Experiment. The primary reason for plotting the data this way was basically to serve as a visual aid that would enable the reader to get a sense of the magnitude of interaction between the conditions of Experiment 1 and 2. As in the previous results sections, The spatial layout of this figure follows that laid out in Figure 29 and 30 and Table 1 with respect to increment extent (refer to Section 6.1 and 6.2 for further details regarding the spatial layout of this figure). The reasoning behind the planned comparisons for the data between the two experiments was described at the end of Section 5.1.

\subsubsection{Planned Comparisons: The Overall Interaction Between Experiments 1 and 2}

Upon casual inspection of Figure 31, there appear to only be a few conditions where the pattern of results appear to interact. However, before the individual comparisons could be carried out, it was important to determine if the overall interaction between the background and no-background experiments for all of the 16 conditions was significant. Thus the first analysis that was carried out here was designed to test the significance of a global interaction between the two experiments. Accordingly, 2 x $16 \times 4$ (2 background 
types by 16 different increment extents by 4 different orientations) three-way repeated measures Analysis of Variance (ANOVA) was carried out. The result of this analysis revealed the interaction between the two sets of data was indeed significant $\left(F_{(45,360)}=\right.$ $3.25, p=.027)$ indicating significant differences in the pattern of results between the two experiments.

\subsubsection{Planned Comparisons: The Individual Interactions Between the Two Experiments}

Since the overall interaction between the data sets from the both experiments was significant, the final set of statistical analyses for this section involved examining whether or not the pattern of results for each individual condition from the background present experiment significantly interacted with their corresponding conditions in the nobackground experiment. Thus, the data from each corresponding condition from Experiment 1 and 2 were subjected to a $2 \times 4$ (background type by test orientation) twoway repeated measures ANOVA. As is apparent from Figure 31, there were only four conditions were the interaction was significant. The significant effects of background by test orientation were found to occur in three the four conditions contained in the top row of Figure 31 (i.e., Conditions 13, 14, and 15 described in Table 1), and in Condition 5 from the left-most column. For the significant conditions contained in the top row, the Fratios were as follows: Condition 13: $F_{(3,24)}=7.45, p=.001$; Condition 14: $F_{(3,24)}=$ $12.99, \mathrm{p}<.001 ;$ and Condition 15: $\mathrm{F}_{(3,24)}=3.61, \mathrm{p}=.028$. For the significant condition contained in the left most column, the F-ratio was: Condition 5: $\mathrm{F}_{(3,24)}=3.04, \mathrm{p}=.048$. All other comparisons did not yield significant effects of background type by test orientation at the $\mathrm{p}<.05$ level. 


\subsubsection{General Summary of the Interactions Between Experiments 1 and 2}

The primary reason for testing the 16 different triangle filter extent conditions in the presence of a $1 / f$ noise background (Experiment 1) and in the absence of the noise background (Experiment 2) was to determine the effect broadband activation, presumably caused by the noise background, had on the perceptual matches of the different triangle increment filter extents at the four test orientations. The three-way repeated measures ANOVA carried out in Section 6.3.1 showed that those perceptual matches did indeed interact, suggesting that the presence/absence of the noise background had a significant impact on the perceptual matches made in the 16 different increment extent conditions. However, the planned comparisons conducted in Section 6.3.2 showed that there were only a few conditions where the noise background had a significant effect on the perceptual matches of the four test orientations. Aside from the lone condition in the leftmost row of Figure 31 (i.e., Condition 5), the conditions where the presence/absence of the $1 / f$ noise background had significant effects were three of the four conditions contained in the top row of Figure 31 (i.e., Condition 13, 14, and 15). The implication here is that when the extent of the triangle increment was broadband with respect to spatial frequency, the presence/absence of the noise background had an effect on those perceptual matches, regardless of the orientation bandwidth of the triangle filter increment. While Condition 16 in the top row appears to interact, especially with respect to the perceptual matches for vertical, the result of the two-way repeated measures ANOVA indicated that it was not significant, although the interaction did approach significance $\left(F_{(3,24)}=1.10, p=.14\right)$. Nonetheless, in general, the presence/absence of the 
broadband noise background mostly affected the perceptual when the triangle filter increment was broadband with respect to spatial frequency.

Before describing the inherent weighting indices that would provide insight as to how the inherent interactions of the biased number of striate units change as a function of the triangle filter increment extent will be carried out, the neural response simulation experiments that were carried out with the three different content biased image sets described in Sections 2.2.2.1 - 2.2.2.3 will be described. Thus the following section is devoted describing how those simulations were carried out, with the subsequent section being devoted to describing the results of those simulations. Finally, the Discussion section of the current study will be devoted to presenting the inherent and dynamic weighting models showing how the $o_{i j}$ and $w_{i j}$ weights (utilized in the proposed striate normalization control model) change as a function of triangle filter increment extents and the different types of content biases of the natural scene imagery mentioned above. 


\section{PROTOCOL FOR MODELING THE THREE DIFFERENT CONTENT-DEPENDENT EFFECTS}

The following sub-sections will describe the three different protocols for modeling the content-dependent effects described in Sections 2.2.2.1 - 2.2.2.3. The purpose for carrying out these protocols was to obtain separate models of how the dynamic weights (i.e., $w_{i j}$ ) in the proposed striate normalization model change as a function of content-bias contained in each of the stimuli used in the above mentioned sections. Once obtained (via the methods described in each of the following sub-sections), the results will be combined with those of Experiments 1 and 2 in order to provide a fairly comprehensive model of human visual processing of orientation in the context of broadband stimuli.

\subsection{Verifying the Content-Dependent Weighting System}

The mechanism of cortical response normalization described in Section 2.3.2 possesses the potential as an effective model of the cortical interactions that potentially underlie the content-dependent effects described in Sections 2.2.2.1 - 2.2.2.3. In accordance with the three effects, the reasoning behind testing this model consists of three parts. First, with respect to the content-dependent effect 1 , if detection of oriented increments of amplitude at the same orientation as the scene's former amplitude bias was hindered due to the stronger weights of dependent neural responses (in the divisive pool) associated with the content bias (in addition to the increased responses due to the overall predominance of 
the image structure at this orientation), then one would expect to find filter response dependencies associated with the content-biased orientation in the joint conditional histograms. Additionally, since the dependency between filter responses vanished when measured against white noise imagery, it is possible that, when the phase angles corresponding to predominant image content were randomized (as mentioned in Section 2.2.2.1), the dynamic weights in the divisive pool would have been greatly reduced, thereby allowing for greater sensitivity (or return to baseline response sensitivity) for detecting oriented increments of amplitude at that orientation. Second, with respect to content-dependent effect 2 , if detection of amplitude increments at off-horizontal orientations was reduced due to stronger weighting of either units tuned to the lower spatial scales (for images with very steep slopes) or units tuned to the higher spatial scales (for images with very shallow slopes), then stronger response dependencies should be observed in the joint histograms obtained from filters tuned to either low or high spatial scales after having been convolved with natural images having very steep or very shallow slopes with respect to imagery possessing $\alpha$ values close to 1.0 . Finally, with respect to content-dependent effect 3 , if the overall improvement of sensitivity for detecting amplitude increments at each of the four orientations results from a reduction of any inhibitory influence from the horizontally tuned units (assumed to be very strong given the evidence for more units tuned to horizontal orientations), than one could expect to find filter response dependencies in the joint histograms that increase with the increase in horizontal content across a series of natural scenes. Likewise, increases in the responses of obliquely oriented filters to horizontal content should also be observed in 
order to provide an account for the general increase in horizontal sensitivity with increasing horizontal content biases.

\subsection{The Neural Response Simulation Protocol}

In order to model the response properties associated with the content-dependent effects summarized in Section 4.0, a neural response simulation protocol was designed in order to replicate the procedures utilized by Wainwright et al. (2001), deviating only in the type of linear basis functions used to simulate neural responses. Specifically, the linear basis functions employed in the current neural response simulation consisted of Gabor functions generated in the Fourier domain. Such a deviation is not extreme when one considers that Gabor functions have long been accepted as resembling the response properties of spatial processing carried out at the level of striate cortex, and it is primarily that fact that a linear basis set composed Gabor functions is most appropriate for use in the neural response simulation protocol utilized in the current study (Marcelja, 1980). At this point it is necessary to highlight two important issues for the simulated neural response modeling procedure. First, the responses of the linear basis set were assumed to mimic the responses of striate units tuned to select ranges of orientations and spatial scales. Second, the extent to which a particular orientation and spatial scale selective unit (labeled the primary filter response) responding to the same spatial content in an image as other similarly tuned units (labeled secondary filter responses) was assumed to 'reflect' the strength of the weight associated with the amount of reduction (applied via divisive normalization) of response that is applied to the primary filter's response. Thus, only the extent of response overlap can be modeled, that is, the suggested release from inhibition 
due to reduced horizontal unit activity associated with the content-dependent effect described in Section 2.2.2.3 cannot be directly modeled in this protocol. However, the proportional increase in response dependencies between horizontally tuned units with increasing horizontal image content can be used as a negative weighting factor which, when applied to units selective for off-horizontal units, can serve to reduce the amount of normalization associated with those units, thereby increasing their relative sensitivity to the spatial content in which they are most optimally tuned.

\subsection{The Neural Response Simulation Basis Set}

As mentioned in Section 7.2, Gabor functions were utilized as the linear basis set for the current neural response simulation protocol. Gabor filters can of course be viewed as a sinusoidal waveform of a particular spatial frequency and relative orientation modulated by a Gaussian envelope, and in the spatial domain can be expressed as:

$$
g(x, y)=e^{-\frac{1}{2}\left[\frac{x^{2}}{\sigma_{x}^{2}+}+\frac{y^{2}}{\sigma_{y}^{2}}\right]} e^{-j 2 \pi\left(u_{0} x+v_{0} y\right)}
$$

In the Fourier domain, a Gabor filter consists of two 2-dimensional Gaussian functions and can be expressed as:

$$
G(u, v)=G_{1}+G_{2}=e^{-\frac{1}{2}\left[\frac{u_{1}^{2}}{\sigma_{u}^{2}}+\frac{v_{1}^{2}}{\sigma_{v}^{2}}\right]}+e^{-\frac{1}{2}\left[\frac{u_{2}^{2}}{\sigma_{u}^{2}}+\frac{v_{2}^{2}}{\sigma_{v}^{2}}\right]}
$$


where $\sigma_{u}=1 /\left(2 \pi \sigma_{x}\right)$ and $\sigma_{v}=1 /\left(2 \pi \sigma_{y}\right)$ are the standard deviation along two orthogonal directions (thus determining the width of the Gaussian envelope along the $x$ - and $y$-axes in the spatial domain), and

$$
\begin{aligned}
& u_{1}=(u-f \cos \theta) \cos \theta+(v-f \sin \theta) \sin \theta \\
& v_{1}=-(u-f \cos \theta) \cos \theta+(v-f \sin \theta) \sin \theta \\
& u_{2}=(u+f \cos \theta) \cos \theta+(v+f \sin \theta) \sin \theta \\
& v_{2}=-(u+f \cos \theta) \cos \theta+(v+f \sin \theta) \sin \theta
\end{aligned}
$$

where $f$ determines the central frequency of the pass band in orientation $\theta$. Of course, we have $u_{0}=f \cos \theta, v_{0}=f \sin \theta$, and $f=\sqrt{u_{0}^{2}+v_{0}^{2}}$.

To better acclimate the current neural response simulation to the characteristic response properties of the visual system, the basis set was designed to contain filters selective for 12 different $\left(0^{\circ}\right.$ to $165^{\circ}$ in steps of $\left.15^{\circ}\right)$ orientations as well as for different spatial frequency bandwidths as identified in oblique masking studies conducted by Wilson (1991) which are in close conjunction with physiological data from Macaque monkey (De Valois, Albrecht, \& Thorell, 1982). The peaks of the different spatial frequency channels that were identified by Wilson (1991) were as follows: $0.8 \mathrm{cpd}$, $1.7 \mathrm{cpd}, 2.8 \mathrm{cpd}, 4.0 \mathrm{cpd}, 8.0 \mathrm{cpd}$, and $16 \mathrm{cpd}$. However, since the highest spatial frequency has an octave range that extends beyond the Nyquist limit of the images selected for the current protocol, only the first five peak frequencies (along with their respective octave bandwidths) were utilized in the current protocol. This basis set was constructed in the 
Fourier domain and consisted of 60 basis filters (i.e., 12 orientations times 5 spatial frequency octaves), only even-symmetric Gabor filters were used in the proposed protocol; examples of the 2- and 3-dimensional spatial profiles are depicted in Figure 32.

\subsection{Simulated Neural Response Procedure}

The following describes the procedures that were utilized in the current neural response simulation protocol for obtaining the neural weights associated with the contentdependent effects described earlier. As mentioned at the beginning of Section 7.2, the idea for obtaining the normalization pool weights to be used in the proposed striate normalization model was based on the methodology carried out by Wainwright et al. (2001). Specifically, the weights obtained from the neural response simulations carried out for the different image sets described in Sections 2.2.2.1 - 2.2.2.3 will be used to show how the dynamic weighting component (i.e., $w_{i j}$ ) of the proposed striate normalization model would change as a function of different types of natural scene content. Thus, current neural response simulation protocol was carried out with three sets of imagery, each selected from the stimulus images used in the experiments conducted by Hansen et al. (2003) and Hansen and Essock (2004a; 2004b) in their non-manipulated form; that is, the current procedure was carried out on the stimulus imagery utilized in those experiments without the presence of any increments made in the Fourier domain. The procedure itself involved two fundamental steps, both of which were carried out using MATLAB version 6.5, and corresponding Image and Signal Processing toolboxes. Step 1 involved two parts, first, for each image, the image size was increased by 'mirroring' or 'folding' the outer 40 pixels of the image on all sides, thus increasing the 
original $512 \times 512$ pixel image by 80 pixels in both the $x$ and $y$ coordinates (see Figure 33 for further details). Since the filtering process (i.e., part 2 of Step 1) produced high filter responses at the edge of the image, the process of mirroring allowed for the central $512 \times 512$ pixel filtered region (i.e., the original image) to be extracted and quantified without the presence of any false edge responses by the filters. The second part of Step 1 involved the filtering process itself. The filtering process itself will be described for a single image and is as follows: 1) the image was subjected to a Fast Fourier transform to obtain the amplitude spectrum for that image, 2) the amplitude spectrum was then, in turn, multiplied by each of the 60 basis filters described in Section 7.3 (this procedure is identical to convolving the image with each of the basis filters in the spatial domain), resulting in 60 filtered spectra, 3) each filtered spectrum was then Inverse Fourier transformed, 4) each resulting spatial image was then cropped to the central $512 \times 512$ pixel region and then normalized to the maximum response value (refer to Figure 33 for further details). Step 2 involved comparing the amount of filter response overlap for each filtered image to each of the other filtered images via 2D joint histograms. That is, for each filtered image, a 2D joint histogram was generated for that filtered image compared to each of the other 59 filtered images, which resulted in a total of 720 joint histograms for each original image. The joint histograms were constructed by counting the number of co-occurrences of pixel values between two filtered images and plotting the results on a $512 \times 512$ matrix with the primary filter (see Section 2.3.I) plotted on the abscissa and the secondary filter (again see Section 2.3.1) plotted on the ordinate, with each column of that matrix being normalized to the maximum value. The resulting $2 \mathrm{D}$ joint histograms were then down-sampled to $41 \times 412 \mathrm{D}$ matrices in order to simplify the further analyses 
(see Figure 33 for further details). Since this process was carried out on a fairly large original stimulus image set, corresponding joint histograms obtained from stimulus images in the same content bias category were averaged.

As mentioned in Section 2.3.2, when the responses of the primary and secondary or 'comparison' filters are highly dependent, that is when the filter responses of the primary filter can predict the variance of the response obtained from the secondary filter, the joint histogram produces a bow-tie shape. Thus, the relative width (or distribution) of the 'wings' of the bow-tie as one moves from the central point of a histogram increase in an almost linear fashion which could be thought of as being representative of the strength of the response dependency between two given filter response pairs (see Figure 34 for further details). That is, considering each vertical half of a given histogram in turn, if the standard deviation of each of the distributions of points (i.e., in each column) around the central line of the histograms was measured, one would expect that, for a joint histogram created from two similar filter response images, the standard deviation would increase linearly (along the $x$-axis, refer to Figure 34 for further details). Thus, the magnitude of the linear relationship between standard deviation within each column and increasing distance (along the $x$-axis) from the center point was used as the weighting factor that would be applied in the proposed model's divisive normalization pool for the contentdependent effects component (i.e., $w_{i j}$ ) of the proposed model. The method for extracting the linear relationship of the wings (or lack thereof) was carried out in three steps. First, in order to reduce the inherent noise in the histograms, each joint histogram was 'folded' about its central point by averaging the left and right sides (both sides were of course 
aligned such that the inner and outer most edges coincided). The result was a $21 \times 41$ matrix, with the left most column containing the central point of the original histogram. Second, for each column of the reduced joint histogram, the standard deviation (unbiased) was taken. Finally, the standard deviations were fit with a regression line (as a function of increasing distance from the central point contained in the left most column of the reduced histogram). The result of this process produced an $R$ value that served as a weight indicating the response dependency between two given filters, thus steeper slopes indicated a high dependency and would thus be a strong weight (see Figure 34 for further details) in the normalization pool of the proposed striate normalization model. The entire procedure described in the current sub-section was of course carried out for all of the original images contained in the three stimulus images sets described in Sections 2.2.2.12.2.2.3.

\subsection{Image Sets Used in Constructing the Content-Dependent Effects Weights}

The content-dependent effects weights were constructed from the same natural scene stimulus imagery described in Sections $2.2 .2 .1-2.2 .2 .3$. However, for computational ease, not all images were used. The following sub-sections are devoted to the specificities of each image set.

\subsubsection{Content-Dependent Effect 1: Fixed Content-Bias Weights}

Content-dependent effect 1 was demonstrated with natural scene imagery that contained phase-defined structural biases at each of the four nominal orientations described earlier. The stimuli used to demonstrate this effect consisted of 4 sets of 8 content biased images 
(with the amount of bias being relatively constant across all sets). Since content dependent effect 1 vanished when the phase coordinates corresponding to the spatial biases were scrambled, any response dependencies observed for each of the 4 sets of the four images should also vanish when the phase coordinates of the biases are scrambled. Thus the procedures described in Section 3.2.1.2 were carried out for the sets of stimulus images that produced content-dependent effect 1 as well as that same set after having had their respective content biases removed via orientation-specific phase scrambling. At this point it is necessary to note that the filtering and subsequent plotting of joint histograms for the orientation-specific images was simply for verification that it is indeed the presence of the fixed content biases contained in those images that are responsible for any observed dependencies depicted in the $2 \mathrm{D}$ joint histograms. Only the non phase scrambled images were used to generate the weights to be used in the modeling of content dependent effect 1 .

\subsubsection{Content-Dependent Effect 2: Variable a Weights}

Content-dependent effect 2 was demonstrated with 6 sets of natural scene imagery (four images per set), where each set possessed images with approximately equal $\alpha$ values, with different $\alpha$ values associated with each set, again, the range of $\alpha$ values for that image set was as follows: $0.659,0.725,0.854,0.949,1.046$, and 1.443 . The response dependencies observed from the images possessing amplitude spectrum slopes equal to 0.949 served as the comparison dependencies since it was with those images that sensitivity for detecting off-horizontal amplitude increments was highest. Thus for the higher response dependencies observed in the higher (0.695 slope images) or lower (1.44 
slope images) spatial frequency ranges, the increase in the $\mathrm{R}$ of the standard deviations of the $2 \mathrm{D}$ joint histogram wings at those ranges was used at the weights associated with content-dependent effect 2 .

\subsubsection{Content-Dependent Effect 3: Variable Horizontal Content Bias Weights}

Since content-dependent effect 3 resulted as a function of the amount of horizontal content present across a series of 15 natural scene images, only those images will be used. In order to limit processing time for constructing the weights associated with content-dependent effect 3 , only six images were selected to generate the weights associated with this effect. Specifically, the original 15 stimulus images were first rank ordered with respect to the amount of horizontal content bias contained in each image (with image 1 being least amount of horizontal content, and image 15 having the largest amount of horizontal bias) after the rank ordering, images $1,3,6,9,12,15$ were selected for the current analyses. The primary difference between the weights associated with content-dependent effect 3 and those from 1 and 2 is that these weights will be negative since overall sensitivity for detecting amplitude increments increased with increasing amounts of horizontal content biases. As mentioned earlier, this assumes that the relative amount of response reduction from the horizontally tuned units is proportional to a release from inhibition from the horizontal units onto the other orientation tuned units. Again, since horizontal sensitivity also improved with the amount of horizontal content bias across the scenes, this increase may likely be a result of the increase in sensitivity (for reasons just described) of units tuned to obliquely oriented content. Indeed, the work of Wainwright et al. (2001) and Schwartz and Simoncelli (2001) has shown high 
response dependencies between units tuned to either horizontally or obliquely oriented stimuli.

\subsection{Results: Content-Dependent Effects Neural Response Simulations}

In the three sub-sections below, the results from the neural response simulations for each of the three content-dependent effects will be presented. From the three neural response simulations: 1) 23,040 joint histograms we created for the image set utilized to model the change in weights for content-dependent effect $1(12 \times 12 \times 5$ filter response comparisons x 32 images), 2) 21,600 joint histograms we created for the image set utilized to model the change in weights for content-dependent effect $2(12 \times 12 \times 5$ filter response comparisons x 30 images), and 3) 4,320 joint histograms we created for the image set utilized to model the change in weights for content-dependent effect $3(12 \times 12 \times 5$ filter response comparisons $x 6$ images). In order to reduce the analyses of these histograms down to a manageable number, only the comparisons (i.e., primary filter to each of the other filters) for the four orientations of interest (i.e., vertical, $45^{\circ}$ oblique, horizontal, and $135^{\circ}$ oblique) were examined from each of the three simulations.

\subsubsection{Neural Response Simulations for Content-Dependent Effect 1}

In the interest of practicality, only the filter comparisons between the filters with a peak response at $2.8 \mathrm{cpd}$ (i.e., scale 3 ), which was treated as the primary filter, and the filters tuned to all other orientations and spatial scales (i.e., scale $1: 0.8 \mathrm{cpd}$, scale $2: 1.7 \mathrm{cpd}$, scale 4: $4.0 \mathrm{cpd}$, and scale $5: 8.0 \mathrm{cpd}$ ), treated as the secondary filters, were subjected to analysis. In addition, only the comparisons with the primary filter (selective for scale 3) 
that was tuned to the orientation of the biased content of the imagery utilized in the current simulation were considered for analysis. In order to provide a more generalized description of the different filter response comparisons, histograms obtained from corresponding comparisons for the eight different images within each of the natural scene content biased images were averaged. For example, for each of the eight images in the $45^{\circ}$ natural scene content bias set, eight joint histograms were produced (one for each image) from the response of the primary filter with a peak response at scale 3 and $45^{\circ}$ oriented content and the secondary filter with a peak response at scale 4 and $15^{\circ}$ oriented content were averaged (refer to Figure 35 for an illustration of this procedure). This procedure would be analogous to averaging the response overlap between two striate cells across eight natural scenes that contained similar content biases. The result of this procedure was a set of averaged filter response comparisons (across corresponding comparisons) across the eight images contained in each of the different content biased image sets. Typical joint histograms obtained from this procedure are plotted in Figure 36. In that figure, averaged joint histograms are plotted that were obtained from comparing a primary filter with peak response at scale 3 for content oriented at $45^{\circ}$ with filters that had peak responses at the other four spatial scales, and for six different orientations. Notice that the strongest averaged response dependencies (prominent 'bowtie' shape) were obtained when the secondary filter responses were more similar to the primary filter with respect to spatial scale and orientation. For comparisons between the primary and secondary filter responses that were less similar with respect to spatial scale and orientation, the responses dependency was less prominent. The next step involved subjecting each of the averaged histograms to the procedures described in Section 7.4 in 
order to the obtain regression slope values that would serve the weights that would be used to model the changes in $w_{i j}$ as a function of natural scene content bias. Since the interest here was to obtain a generalized description of response dependency between the differently tuned filters when passed over content biased imagery, the weights obtained from the procedure described in Section 7.4 were aligned with respect to peak response of the primary filters and averaged (refer to Figure 35 for further details regarding this procedure). The reasoning here is that since the filters respond in a linear fashion, any differences observed between the obtained weights would be a function of the imagery and not of the filters themselves. While such an analysis is not without merit, the four sets of different oriented content biased imagery is by no means a complete description of all biased images one might encounter (after all, there were only eight images per content bias type). Thus, by aligning the responses with respect to peak response of the primary filters the question becomes, for any typical orientation bias encountered in a natural scene, how are the striate neural units tuned to that biased orientation weighted within a network of cortical units tuned to all other orientations and spatial scales. The results of the entire process described above are plotted in Figure 37. Note that this figure has been smoothed by bicubic interpolation. The color bar for this figure indicates which colors were plotted according to the regression line slopes for the different averaged joint histograms. This plot provides a quantitative description of the pattern of response dependency changes alluded to in Figure 36. Specifically, filters selective for spatial scales and orientations similar to that of the primary filter yield higher $w_{i j}$ values then filters that are tuned to spatial scales and orientations less similar to that of the primary filter. On closer inspection of Figure 37, one can see that the primary filter is weighted 
most by the responses of filters that are tuned to orientations that differ from the primary by $\pm 30^{\circ}$ at scales 2 and 4 . Further discussion of these results will be given in the Discussion section of the current study.

\subsubsection{Neural Response Simulations for Content-Dependent Effect 2}

The primary reason for carrying out the neural response simulations with the variable amplitude spectrum slope imagery was to determine whether or not the over all magnitude of the neural response dependencies (regardless of orientation) changed in proportion to the slope of the amplitude spectrum fall-off. That is, given the pattern of results observed by Hansen and Essock (2004b) and described in Section 2.2.2.2, one might expect to find that for imagery with shallower amplitude spectrum slopes, the magnitude of the response dependencies between the filters tuned to higher spatial frequencies would be larger (compared to the response dependencies from filters tuned to lower spatial scales, as well as those observed with imagery possessing steeper amplitude spectrum slopes). Likewise, the magnitude of the filter response dependencies would be higher for the filters tuned to lower spatial scales with imagery possessing very steep amplitude spectrum slopes (compared to the response dependencies from filters tuned to higher spatial scales, as well as those observed with imagery possessing more shallow amplitude spectrum slopes).

The results from the current simulation yielded 21,600 joint histograms. In order to organize those histograms into a meaningful description of how the response dependencies changed with amplitude spectrum slope, the following procedure was 
carried out. First, all corresponding histograms within a given image set were averaged. The next step involved subjecting the averaged histograms to the procedures outlined in Section 7.4 in order to obtain the regression slope values that served as indicators of the magnitude of the response dependencies between the different filter comparisons. The final step involved averaging those values with respect to spatial scale of the primary filter. That is, all regression line slops were averaged across each orientation of a primary filter compared to all other secondary filters with a peak response at the same scale. This procedure resulted in a single value for each of the five examined spatial scales for each of the six amplitude spectrum slope image sets. The results from this procedure are plotted in Figure 38. Note that this figure has been smoothed by bicubic interpolation. The color bar for this figure indicates which colors were plotted according to the regression line slopes for the different averaged joint histograms. Notice that the averaged filter responses for each spatial scale definitely change as a function of the slope of the amplitude spectra of the imagery utilized in the current simulation. For the images in the 0.659 slope set (i.e., the shallowest slope set), the magnitude of the averaged neural response dependencies are highest at the highest spatial scales, in fact, these were the highest values obtained across all image types. For the images in the steeper slope sets (i.e., 1.046 and 1.443), the magnitude of the averaged neural response dependencies are highest at the lowest spatial scales, which were the second and third highest values obtained across all image types. Finally, for the images in the slope sets between the extremes (i.e., $0.725,0.854$, and 0.949 ), the magnitude of the averaged neural response dependencies were, overall, the lowest. How these weights will play into the proposed 
striate normalization model will be addressed further in the Discussion section of the current paper.

\subsubsection{Neural Response Simulations for Content-Dependent Effect 3}

The fundamental question for the current set of simulations was to determine whether of not the filter response dependencies where the primary orientation was horizontal increased across a set of natural scene imagery that contained an increasing amount of horizontal content bias across images. Thus, it is the dependencies between the primary filter (with a peak response at horizontal) and the other filters tuned to orientations other than horizontal that is important. In the interest of practicality, only the response dependencies between a primary filter tuned to scale 3 (and selective to horizontal) and filters tuned to each of the other spatial scales and orientations were examined here.

The procedure utilized in the current analysis was as follows. First all histograms where the primary filter's orientation was vertical and spatial scale was scale 3 were selected. Next, for each of the six horizontal content biased images, all corresponding histograms were averaged across spatial scale of the secondary filter with respect to the orientation of the comparison filter. For example, for a given image, joint histograms where the primary filter was compared to secondary filters with peak responses to each one of the four other spatial scales as well as being selective for vertical orientations were averaged. The final step involved subjecting the averaged histograms to the procedures outlined in Section 7.4 in order to obtain the regression slope values that served as indicators of the magnitude of the response dependencies between the different filter 
comparisons. The result of this process yielded 12 regression slope values for each of the six images, all of which are plotted in Figure 39. Note that this figure has been smoothed by bicubic interpolation. Along the ordinate are the rank-ordered percentages of local filter responses (Gabor filters) tuned to horizontal for each image. These values were obtained by the methods described in Section 2.2.2.3. Essentially, Gabor filters that were aligned with horizontal were convolved with each of the six images utilized in the content-dependent effect 3 neural response simulation. Next, the total number of pixel values in the filtered images that fell outside of $\pm 1 \mathrm{SD}$ of the pixel distribution of each filtered image were summed. The ratio of this sum to the total number of pixels in each of the filtered images was taken as the local horizontal response bias for each image. Thus, it is the percentage of response bias for each of the six images that is plotted on the ordinate of Figure 39 (refer to Section 2.2.2.3 and Hansen and Essock (2004b) for further details regarding this process). The color bar for this figure indicates which colors were plotted according to the regression line slopes for the different averaged joint histograms. From this figure it is clear that as one moves from lower horizontal biases (plotted on the ordinate with respect to increasing amount of horizontal content), the weights obtained from response dependencies between the primary filter (which was tuned to horizontal) and secondary filters selective to orientations within $\pm 60^{\circ}$ (averaged across spatial scales other than that of the primary filter) increase rapidly. For secondary filters tuned to orientations very different from horizontal (i.e., $\geq 75^{\circ}$ ), the response dependencies remain approximately equal. The significance of these weights with respect to the proposed striate normalization model will be addressed further in the Discussion section of the current paper. 


\subsubsection{Neural Response Simulation General Results}

The results from the three different neural response simulations obtained from the three different image sets, described in Sections 7.5.1 - 7.5.3, yielded a system of weights that, when implemented into the striate normalization model could reasonably explain the different patterns of results obtained from the psychophysical experiments reviewed in Sections 2.2.2.1 - 2.2.2.3. How these weights will be implemented into the proposed model will be addressed in the Discussion section of the current study. In the current sub-section, only the results from the three neural response simulations reported above will be reviewed. The results from the neural response simulations carried out on the content-dependent effect 1 image set produced a system of weights that showed that the response of a given primary filter is weighted most by the responses of filters that are tuned to orientations that differ from the primary by $\pm 30^{\circ}$ at scales 2 and 4 . In addition, those weights were obtained by aligning the primary filters so that the weights could be used with respect to any orientation that a content bias is exhibited in a given natural scene. The results from the neural response simulations carried out on the contentdependent effect 2 image set showed that whatever orientation where content bias induced response dependencies are exhibited, the overall magnitude of the dependencies (i.e., the weights assigned to $w_{i j}$ ) will vary as a function of the amplitude spectrum slope of the natural scene. However, as the results described in Section 2.2.2.2 suggest, the response dependency weights are extremely strong at either the higher spatial scales when the imagery had very shallow amplitude spectrum slopes, or at the lower spatial 
scales when the imagery had very steep amplitude spectrum slopes. When the imagery had amplitude spectrum slopes that were intermediate, the response dependency weights were the lowest. Finally, the results from the neural response simulations carried out on the content-dependent effect 3 image set showed that the response dependencies between a given horizontal filter and secondary filters tuned to other orientations and spatial scales would increase across a set of natural scene images that contained increasing amounts of horizontal content. 


\section{GENERAL DISCUSSION}

The general outline for the current discussion section will first involve the results obtained from the two psychophysical experiments, followed by a discussion of the results from the three different neural response simulations. The final portion of the current discussion section will be focused on incorporating the results from the two psychophysical experiments and the three neural response simulations into the proposed striate normalization model, along with a schematic representation of the model.

The fundamental reason for conducting Experiments 1 and 2 was to examine suprathreshold sensitivity for varying amount of oriented content either within a broadband $1 / f$ noise background or alone. There are two primary ways in which the data from those experiments can be discussed. The first part (Part-I) of the psychophysical experiment discussion (i.e., all sub-sections in Section 8.1) will involve the relative changes in the patterns of suprathreshold sensitivity between the four different orientations within each condition, across all conditions (i.e., as a function of the triangle increment extent). The focus of that discussion will be on the gradual shift from the oblique effect pattern of results for the more limited triangle increment extents (i.e., narrow spatial frequency and/or narrow orientation increment bandwidths) to the horizontal effect pattern of results for the more broad triangle increment extents (i.e., 
broad spatial frequency and/or broad orientation increment bandwidths). The first set of results that will be discussed for Part-I of the psychophysical experiments will be those from the no-background condition due to the fact that the conditions in that experiment demonstrated how differently tuned striate neurons might interact with one another as more and more spatial frequencies and orientations are added to a single sinusoidal grating (which was utilized in Condition 1 of that experiment). Accordingly, the results from that experiment will be discussed by treating Condition $I$ as a reference point to which the pattern of results obtained from the conditions possessing increasingly larger triangle filter increment extents will be compared. The results from the $1 / f$ noise background experiment will then be discussed with respect to the observed changes between the corresponding conditions in the no-background experiment.

The second part (Part-II) of the psychophysical experiment discussion (i.e., all subsections in Section 8.2) will involve an analysis of the changes in suprathreshold sensitivity for each of the four test orientations as a function of the triangle increment extent for the no-background and the noise background experiment. In order to facilitate the discussion, the data will be 'transformed' into weighting indices for each orientation as a function of the extent of the triangle increment filter. Generating these indices serves two purposes, the first is to allow insight into how suprathreshold sensitivity for each orientation of the test increment changes as a function of the triangle increment filter's extent, the second is to provide an index of inherent weights that can be implemented into the proposed striate normalization model (i.e., the $o_{i j}$ component of the proposed normalization model). The first set of inherent weighting indices that will be discussed 
for Part-II of the psychophysical experiments discussion will be those generated from the no-background condition for the same reasons mentioned above. Accordingly, the inherent weighting indices from the $1 / f$ noise background experiment will then be discussed with respect to the observed changes between the corresponding conditions in the no-background experiment.

Finally, the second portion of the current discussion section (i.e., all sub-sections included in Section 8.3) will involve the results from the three different neural response simulations. Since the primary reason for carrying out those simulations was to provide a dynamic weighting system that could be implemented in the proposed striate normalization model, the data will be 'transformed' into a series of weighting indices that will be implemented into the proposed normalization model (i.e., the $w_{i j}$ component of the proposed striate normalization model). These weighting indices will also allow for a functional description for the pattern of results of the psychophysical data reviewed in Sections 2.2.2.1-2.2.2.3.

The final section of the current discussion (i.e., all sub-sections included in Section 8.4) will be devoted to implementing the inherent weighting indices obtained from the psychophysical experiments and the dynamic weighting indices obtained from the three different neural response simulations into the proposed striate normalization model. As mentioned in the Introduction, there are two primary reasons for implementing the inherent and dynamic weighting indices as an indexing system that will be drawn upon by the proposed normalization model. The first reason is to equip the proposed 
normalization model with inherent weighting indices that allow for 'sensitivity' adjustments as a function of the amount of energy at a given orientation either alone or in the presence of additional broadband energy at all other orientations (e.g., $1 / f$ visual noise patterns). The second reason is to further equip the proposed model with dynamic weighting indices that adjust the output of the proposed model as a function of the type of content contained in typical natural scenes. The end result is a model of cortical gain control in the human striate cortex that is optimally suited to describe the output of the human visual system as it encounters real-world stimuli.

\subsection{Implications from the Current Psychophysical Experiments}

\subsubsection{Implications from the No-background Experiment}

For Condition 1 of the no-background experiment, suprathreshold sensitivity was assessed for a $16 \mathrm{cpd}$ sinusoidal grating at four different orientations. As can be seen from Figure 30, the typical oblique effect pattern of results was obtained. When more sinusoidal gratings were added at different orientations (i.e., the conditions along the bottom row of Figure 30) the oblique effect was still present in most conditions with the exception of the Condition 2 where all four test orientations were perceived as being equivalent to their standard patterns. When orientation bandwidth was held constant at $16 \mathrm{cpd}$, but more and more spatial frequencies were added to that grating (i.e., the conditions within the left-most column of Figure 30) the oblique effect pattern of results was again obtained, with the exception of Condition 13 where the oblique orientations were still elevated above the other two orientations, but horizontal was much more 
elevated than vertical. It could be argued that horizontal was also elevated more than vertical in Condition 4 (i.e., the broad orientation bandwidth, single spatial frequency condition), but the magnitude of this elevation is not as large as that observed in Condition 13. What this suggests is that when more and more spatial frequencies are added at a single orientation, the responses of the horizontally tuned units in striate cortex demonstrate their numerical bias by contributing more input to the normalization pool that adjusts the sensitivity to the horizontal orientation, resulting in reduced sensitivity at that orientation (more pooling here is equivalent to a stronger $o_{i j}$ weight). What this amounts to is that the bias in the number of striate neurons tuned to horizontal is very closely tied to the horizontal orientation and becomes much more prevalent as more spatial frequencies are included in the test pattern at that specific orientation. While a slight elevation in the averaged horizontal matches (equivalent to a decrease in horizontal sensitivity) can be seen as more and more orientations were added to the single spatial frequency grating, it is very weak, suggesting that the bias in the number of horizontally tuned striate units lies mainly in the neurons tuned strictly to horizontal and is distributed more across the different spatial frequencies. However, this does not mean that that is the primary dimension containing all of the numerical bias of horizontal tuned neurons because while horizontal is elevated in the single orientation and broadband spatial frequency increment extent, oblique sensitivity is still worst in that condition. As the orientation bandwidth of the increment filter is increased in the broadband spatial frequency conditions (i.e., the top row of Figure 30), the horizontal effect becomes much more prominent, arguing that the full numerical bias of horizontal tuned striate neurons exists across a range of orientations centered around horizontal for the full range of 
spatial frequencies examined in that experiment. Indeed, as one observes the different patterns of matches across the top two rows (Conditions $9-12$ and $13-16$ ) or the right two columns (Conditions 3, 7, 11, and 15 and Conditions 4, 8,12 and 16) there is a general transition from some form of an oblique effect to a horizontal effect, where Conditions $6-8$ and $10-12$ being essentially transitional 'null' conditions where essentially no effect can be observed.

In Section 4.0 it was suggested that the Wainwright (2001) model be made to posses an 'inherent weighting' component (i.e., $o_{i j}$ ) that would serve to scale the nearest neighbor responses at various orientations according to the numerical bias of neurons tuned to different orientations. The results of the no-background experiment suggest that the weights assigned to horizontally tuned striate units are elevated more (which in turn acts to reduce sensitivity to horizontal content) along the spatial frequency dimension for neurons tuned strictly to horizontal content than for neurons strictly tuned to $16 \mathrm{cpd}$ and orientations at or near horizontal. However, it is not until neurons selective to horizontally oriented content across the full range of spatial frequencies examined in the current experiment do the weights associated with the normalization pool of the horizontally tuned neurons become extremely large (potentially leading to the horizontal effect pattern of suprathreshold matches observed in that experiment). In subsequent sections the data described above will be transformed into a model weighting index for $o_{i j}$ that could be drawn upon by the proposed striate normalization model in order to weight output responses based on the amount of oriented content (created here by a triangle increment of amplitude) presented alone. Thus, this index serves only to describe how 
proposed model weighting system behaves in and of itself. Specifically, it describes the 'pure' form of the weighting system for a population of neurons that exhibit a numerical bias with respect to orientation selectivity. While such a description is not without merit, it does not describe how the system of weights would change under conditions of 'broadband activation'. That is, when a triangle increment is embedded in a broadband noise background (which, as mentioned in the Introduction, closely resembles the energy distribution across orientation and spatial frequency of natural scenes), where all striate units are activated by the noise pattern, the interaction of the broad activation on the units tuned to the orientation of the triangle filter is not described in the weighting system described above. Since any given natural scene possesses energy at all spatial scales and orientations, it would be useful to have a description of the weighting system when triangle increments of amplitude at different orientations and spatial scales are embedded in a pattern that would activate the system in a manner similar to natural scenes. Thus, a system of weights needs to be devised to explain how the different weights for the different output units would change for oriented increments embedded in broadband images. The conditions examined in the broadband $1 / f$ noise pattern experiment provided data that would allow for such a weighting system to be devised. The following sub-section is thus devoted to discussing the results of that experiment with respect to those obtained from the no-background experiment in order to devise a more 'realistic' description of the $o_{i j}$ weighting index. 


\subsubsection{Implications from the $1 / f$ Noise Background Experiment}

As in the no-background experiment, for Condition 1 of the noise background experiment, suprathreshold sensitivity was assessed for a 16cpd sinusoidal grating at four different orientations. As can be seen from Figure 31, the typical oblique effect pattern of results was obtained that was little different from that obtained in the no-background experiment. This finding is not a trivial due to the fact that, until now, it was not known whether or not such a pattern of results would be obtained for high spatial frequency sinusoidal gratings embedded in broadband $1 / f$ noise. When the extent of the increment was increased with respect to orientation (i.e., the conditions along the bottom row of Figure 31) the oblique effect was still present in most conditions with the exception of the Condition 2 where all four test orientations were perceived as being equivalent to their standard patterns. Thus, for this set of conditions, the presence of a broadband noise background had no effect on the pattern of suprathreshold matches. When orientation bandwidth of the increment was held constant at $16 \mathrm{cpd}$, but more and more spatial frequencies were added to that grating (i.e., the conditions within the left-most column of Figure 31) the oblique effect pattern of results was again obtained as they were in the corresponding conditions of the no-background experiment. However, the oblique effect pattern of results obtained form Condition 13 of the noise background experiment deviate significantly from the pattern of results in the corresponding condition of the nobackground experiment. Specifically, the average horizontal match was not elevated relative to the average vertical match, and the average vertical match is much closer to the average oblique matches compared to the no-background condition. In fact, there does not appear to be any effect of adding additional orientations or spatial frequencies to 
the increment across the conditions in the bottom row or left-most column in the noise background experiment plots in Figure 31. With the exception of Condition 2, the bottom row and left-most column generally exhibit an oblique effect pattern of results, without any major indications of the presence of a horizontal effect. This was not the case in the left-most column for the no-background plots in Figure 31, where the average horizontal match was significantly elevated in Condition 13. Thus, it appears as though the broadband activation caused by the noise background served to disinhibit the striate units specifically tuned to horizontal orientations.

The next significant deviation from the no-background experiment exhibited by conditions in the noise background experiment can be observed by examining the top row in Figure 31. The differences between the two Condition 13 pattern of results have already been addressed. Notice that Condition 14 in the no-background experiment begins to show a somewhat large horizontal effect where the corresponding condition in the noise background experiment is essentially 'null', showing no differences between the four orientations. Furthermore, while Conditions 15 and 16 show predominant horizontal effects, the average of the vertical matches in the noise background experiment is much more elevated compared to the average of the vertical matches in the no-background experiment. Notice that the vertical matches in those no-background conditions are very close to 1.0 , indicating that the nine participants generally accurately matched the strength of the vertical increments to those of the standard. However, in the presence of a broadband noise background, the vertical matches were more elevated (i.e., reduced suprathreshold sensitivity). This finding is very much in line with the physiological 
evidence that suggests that in addition to a larger number of horizontally tuned striate neurons, there are also a larger number of vertically tuned striate neurons relative to obliquely tuned striate neurons. Thus, as more and more vertical neurons are activated by the increasingly large triangle filter extent, the weights in the normalization pool associated with vertically tuned neurons increases. However, if this were true, one would expect to find elevated vertical matches in the no-background experiments, which was not the case. One potential explanation for this difference may lie in the pooling extent associated with the vertically tuned neurons. That is, it may be the case that under conditions of broadband activation, the extent of the normalization pool associated with vertically tuned units is expanded to include neurons only slightly tuned to vertical, but this is pure speculation. Currently, the difference between the vertical matches of both experiments cannot be explained. However, along with the slower decrease in suprathreshold sensitivity across the top row, the differences between the vertical matches arise out of the interaction between those cortical units and the broadband activation caused by the $1 / f$ noise background.

The last issue to address with respect to the effects of the broadband noise background is the 'rate' at which the participants shifted from an oblique pattern of results to a horizontal effect pattern of results. Notice that the different patterns of matches across the top two rows (Conditions $9-12$ and $13-16$ ) or the right two columns (Conditions 3, 7, 11, and 15 and Conditions 4, 8,12 and 16) exhibit a general transition from some form of an oblique effect to a horizontal effect, where Conditions $6-8$ and $10-12$ being essentially transitional 'null' conditions where essentially no effect can be observed. The 
fundamental difference between the two sets of experimental conditions lies in the fact that the noise background experiment exhibits little difference in the shift with respect to increasing spatial frequencies or orientations contained in the increment. Specifically, it appears as though both spatial frequency and orientation bandwidth need to be significantly increased before a prominent horizontal effect can be observed.

\subsection{The Weighting Indices for the Proposed Striate Normalization Model}

In the following sub-sections, the weighting indices derived from the data obtained in the two psychophysical experiments and the results from the three neural response simulations will be made explicit. The primary reason for constructing these indices will be to simply show how those weights change as a function of different types and amounts of oriented content change in a given broadband image (e.g., visual noise of natural scene imagery). After presenting the indices, a demonstration will be provided that illustrates how those weights would be drawn upon and combined in the proposed striate normalization model.

\subsubsection{The Inherent Processing Bias Weighting Indices}

In Section 4.0 it was suggested that the Wainwright (2001) model be made to posses an 'inherent weighting' component (i.e., $o_{i j}$ ) that would serve to scale the nearest neighbor responses at various orientations according to the numerical bias of neurons tuned to different orientations. The primary question left by this suggestion was whether or not this weight should be a constant factor, or made to vary with the overall amount of oriented structure contained in a given broadband image. Thus, if sensitivity to different 
amounts of incremented oriented content changed as a function of the amount of oriented content incremented in a set of broadband noise images, then one could assume that if the proposed striate normalization model was representative of striate processing, the inherent weighting factor would change as a function of the amount of incremented oriented content. Specifically, a determination needed to be made regarding the proposed adjustment to the Wainwright (2001) model for how the different weights would be applied to the responses of striate neurons tuned to different ranges of spatial frequencies and orientations. Thus, it would be useful to know exactly how these weights change in the normalization pool of the proposed model as a function of stimulus content-bias as well as a function of the extent of the increment in the Fourier domain. In order to show how the inherent weights (i.e., $o_{i j}$ ) might change as a function of increment extent (that is, extent in the Fourier domain), four indices were generated from the data obtained from each of the two psychophysical experiments, one for each of the four test orientations.

In order to present a meaningful plot of the different $o_{i j}$ weights from each of the conditions within each of the two experiments, the following procedures were carried out. First, for Experiment 1, all of the averaged scalar ratios for each orientation were assembled into separate data matrices organized with respect to the triangle filter extent in the exact same fashion as the graph matrices shown in Figures 29 - 31 (i.e., Condition $l$ was in the bottom-left cell, etc.). For each orientation, the matrix of increment scalar ratios was flipped (i.e., copied) right-left about the Condition 1 scalar ratio. Then the new 'expanded' matrix for each orientation was then flipped bottom-up. The result for each orientation was a matrix of scalar ratios that was twice as large as the original 
matrix. The data matrices for each orientation were then smoothed using bicubic interpolation. Each orientation matrix was then plotted in 3D coordinates and mapped to a color space where higher scalar ratios are represented by red and low values represented by blue, all values between were mapped to shades of green, yellow, and orange (i.e., the same color mapping utilized for Figures 37-39). The same process was of course carried out for results of Experiment 2. The indices for each orientation in Experiment 1 are shown in Figure 40, and from Experiment 2 in Figure 41. Note that the $x$ and $y$ axes are split with respect to spatial frequency ( $y$-axis) and orientation ( $x$-axis) of the triangle filter increment, where $16.0 \mathrm{cpd}$ indicates the series of conditions where the spatial frequency extent of the increment was single - and where, on the $x$-axis, either $0,45,90$, or 135 Single indicates the series of conditions where the orientation bandwidth of the increment single and centered on one of the four test orientations. Plotted at the center position of each plot is the magnitude of the scalar ratio for Condition 1. In order to present the inherent weighting indices more clearly, the original index for each orientation has been folded (i.e., copied) right-left (effectively doubling the length of the index) and then folded bottom-up (effectively doubling the height of the index). As one moves away from the center along either the $x$ (orientation bandwidth) or $y$ (spatial frequency bandwidth) axis, the magnitude of the scalar ratio for that particular triangle increment extent can easily be examined relative to the other triangle filter extents. Note that values greater than 1.0 on those plots indicate poor suprathreshold sensitivity; values near 1.0 indicate accurate suprathreshold sensitivity; and values below 1.0 indicate 'heightened' suprathreshold sensitivity. 


\subsubsection{The No-Background Inherent Weighting Indices}

Careful examination of the top-view weighting indices in Figure 41a and 41b provides further insight into the effects of suprathreshold sensitivity as the triangle increment filter was made to include different numbers of spatial frequencies and orientations. The most obvious feature that stands out upon examining those plots is that the changes in the inherent weights for both oblique orientations (i.e., Figure 41b) across the different triangle increment extents are very similar. Specifically, as the spatial frequency bandwidth of the triangle filter is increased along the $45^{\circ}$ and $20^{\circ}$ orientation filter bandwidth grid line, non-monotonic functions of the inherent weights were observed where the weights start out rather high, then decrease in the $1 / 2$ to 1.0 octave range, followed by increases toward the broadband spatial frequency filter extent condition. What this amounts to is that suprathreshold sensitivity in those conditions (for both of the obliquely oriented triangle increments) starts out very poor, gradually increasing as spatial frequencies are included in the increment, followed by a decrease in sensitivity toward the broadband frequency condition. Additionally, increasing monotonic functions are observed along the $5^{\circ}$ and single orientation filter bandwidth grid line as spatial frequencies are included in those filter extents. Thus for both of the obliquely oriented test increments, suprathreshold sensitivity starts out relatively high and gradually decreases as more spatial frequencies are included in the filter extents. Another similarity between the plots of the inherent weights for both of the obliquely oriented test increment conditions can be seen along the $1 / 2,1.0$, and broadband spatial frequency increment extent grid lines as the orientation bandwidth of the increment was increased. Specifically, along those axes, both of the obliques show decreasing monotonic functions 
(that decrease at different rates) with the inherent weights starting out relatively high and decreasing with increasing orientation bandwidth of the increment extent. Thus, suprathreshold sensitivity in those conditions starts out very poor and gradually increases with increasing orientation bandwidths of increment extent. Additionally, both of the obliques show non-monotonic functions along the single spatial frequency grid line that start out relatively high, followed by a decrease around the $5^{\circ}$ increment bandwidth coordinate, followed by an increase across the $20^{\circ}$ and $45^{\circ}$ orientation bandwidth coordinates. The relative similarity between the patterns of changes of the inherent weights for both of the obliquely oriented test increments suggests that the nature in which the striate units, tuned to either of the oblique orientations, are pooled in the normalization pool associated with each of those orientations is very similar. Also apparent in the graphs is that the inherent weights are highest (i.e., low inherent weights in the normalization pool) when the triangle filter was made to contain an octave's worth of spatial frequencies (where 1.0 octave possessed spatial frequencies in the $8-16 \mathrm{cpd}$ range) and orientations in the $5^{\circ}$ to $45^{\circ}$ range. Thus, in those increment extent conditions, striate 'neurons' in the normalization pool do not appear to interact.

The second obvious feature that arises out of Figure 41 (top-view graphs) is that the changes in the inherent weights between the two cardinal orientations (i.e., Figure 41a) across the different triangle increment extents are very different. In fact, with the exception of the single orientation- $20^{\circ}$ orientation bandwidth increment extent coordinate, there appears to be very little change in the inherent weights for the vertically oriented increment extents. Whether or not that point in the vertical weighting index is 
'real' or not must be considered in the context of the weights for the other three orientations. That is, that condition elicited overall high scalar matches compared to all of the other increment extent conditions. Admittedly, this issue may be related to the relative magnitude of the standard pattern increment in that condition compared to the increments of the standards of the other conditions. However, when all of the weights for that condition are normalized to vertical, the other three orientations still exhibit relatively high weights for that condition. Regardless of that issue, most of the vertical weighting index is approximately in the 1.0 range, suggesting that, for the most part, vertical was accurately perceived in all of the increment extent conditions. This observation is contrasted by the dramatic changes in the inherent weights observed in the horizontal weighting index for the different increment extents. Close examination of the horizontal weighting index for the different orientation bandwidth increment extent grid lines, along which the spatial frequency bandwidth of the increment was made to vary, shows a series of non-monotonic functions of the inherent weights (all of which change at different rates). Specifically, when the increment extent consisted only of a single frequency, the weights are relatively high, the inherent weights then decrease as more and more spatial frequencies are included in the increment, this decrease is followed by a dramatic rise in the 1.0 octave to broadband spatial frequency range. As indicated earlier, what this means is that suprathreshold sensitivity follows a non-monotonic function that is inverse to the weights. However, examining the different spatial frequency bandwidth increment extents, where orientation bandwidth of the increment was made to vary, increasing monotonic functions for the inherent weights are observed as orientation bandwidth of the increment filter is increased (with the exception of the 1.0 octave 
condition). The dramatic difference between the two cardinal orientation weighting indices indicates that the nature in which the neural units associated with those orientations is drastically different. For vertical orientations, there appears to be little interaction between the striate 'neurons' in the normalization pool associated with vertical orientation processing. For horizontal orientations however, the interaction between the striate 'neurons' in the normalization pool associated with horizontal processing is very strong, especially in the conditions where the increment extent is very broad. The latter pattern of inherent weight changes very much relates to the numerical bias of horizontally tuned striate units mentioned in the Introduction. That is, since there are more neurons tuned to horizontal, as more and more of those units are activated, their collective weight in the horizontal normalization pool is larger than the other three orientations, thereby decreasing sensitivity to broadband (i.e., spatial frequency and orientation) horizontal orientations. However, while more horizontally tuned neurons have been observed in striate cortex relative to vertically tuned neurons, a bias in the number of vertically tuned neurons (relative to obliquely tuned neurons) has also been observed in striate cortex. Thus one would expect to observe elevated inherent weights in the vertical index for the conditions where the triangle increment extent was very broad. It may very well be the case that, while there is a bias in the number of vertically tuned striate units, that the bias is simply not great enough to evoke large pooled weights in the vertical normalization pool. This issue will be revisited in Section 8.2.1.3 where the weighting indices generated from the data obtained from Experiments 1 and 2 are compared. 


\subsubsection{The Noise Background Inherent Weighting Indices}

As with the top-view weighting indices in the preceding section, those in Figure 40a and 40b also provide further insight into the effects of suprathreshold sensitivity as the triangle increment filter was made to include different numbers of spatial frequencies and orientations. Of course, here those increments were embedded in broadband $1 / f$ noise. The most obvious feature that stands out upon examining those plots is that the changes in the inherent weights for both oblique orientations (i.e., Figure 40b) across the different triangle increment extents are very similar. Specifically, as the spatial frequency bandwidth of the triangle filter is increased along the $45^{\circ}$ and $20^{\circ}$ orientation filter bandwidth grid line, the inherent weights remain approximately equal (i.e., they contain no strong increases or decreases in the inherent weights along those axes). What this translates to is relatively constant suprathreshold sensitivity in those conditions (for both of the obliquely oriented triangle increments). Additionally, increasing monotonic functions are observed along the $5^{\circ}$ and single orientation filter bandwidth grid line as spatial frequencies are included in those filter extents. Again, this amounts to suprathreshold sensitivity for obliquely oriented test increments that starts out relatively high and gradually decreases as more spatial frequencies are included in the filter extents. Another similarity between the plots of the inherent weights for both of the obliquely oriented test increment conditions can be seen along the single and broadband spatial frequency increment extent grid lines where the orientation bandwidth of the increment was increased. Specifically, along those axes, both of the obliques show non-monotonic functions (that change at different rates) with the inherent weights starting out relatively high, followed by a decrease at the $5^{\circ}$ orientation bandwidth coordinate, followed by a 
slowly increase in the weights across the $20^{\circ}$ and $45^{\circ}$ orientation bandwidth coordinates. Additionally, both of the obliques show decreasing monotonic functions along the $1 / 2$ and 1.0 octave grid lines that start out relatively high at the single orientation coordinate which then decreases as the orientation bandwidth of the increment is increased. As for the no-background weighting indices, the relative similarity between pattern of changes of the inherent weights for both of the obliquely oriented test increments suggests that the nature in which the striate units, tuned to either of the oblique orientations, are pooled in the normalization pool associated with each of those orientations is very similar.

The second obvious feature that arises out of Figure $\mathbf{4 0}$ (top-view graphs) is that the changes in the inherent weights between the two cardinal orientations (i.e., Figure 40a) across the different triangle increment extents are also very similar, differing mainly in magnitude. Close examination of the vertical and horizontal inherent weighting indices reveals that for the different orientation bandwidth increment extent grid lines, along which the spatial frequency bandwidth of the increment was made to vary, shows a series of increasing monotonic functions of the inherent weights (all of which change at different rates). Specifically, when the increment extent consisted only of a single frequency, the weights are relatively low, the inherent weights then increase as more and more spatial frequencies are included in the increment. Note that in both of the vertical and horizontal inherent weighting indices, the monotonic increase is very large (more so for the horizontal inherent weighting index) along the $20^{\circ}$ and $45^{\circ}$ axes for the broader spatial frequency coordinates. As indicated earlier, what this means is that suprathreshold sensitivity follows a decreasing monotonic function as spatial frequency is 
increased along the different orientation bandwidth axes for both orientations. However, examining the different spatial frequency bandwidth increment extents, where orientation bandwidth of the increment was made to vary, a series of functions for the inherent weights are observed that start out as decreasing monotonic functions (i.e., the single spatial frequency axes) and gradually 'switch' over to increasing monotonic functions (for the other three spatial frequency axes) as orientation bandwidth of the increment filter is increased. Again, note that in both of the vertical and horizontal inherent weighting indices, the monotonic increase is very large (more so for the horizontal inherent weighting index) along the 1.0 octave and broadband spatial frequency axes for the broader orientation bandwidth coordinates. The relative similarity between the pattern of changes of the inherent weights for both of the cardinal orientation test increments suggests that the nature in which the striate units, tuned to either of these orientations, are pooled in the normalization pool associated with each of those orientations is very similar. For horizontal orientations however, the interaction between the striate 'neurons' in the normalization pool associated with horizontal orientation processing appears to be much stronger than that associated with vertical orientation processing. The latter pattern of inherent weight changes for both of the cardinal orientations very much relates to the numerical bias of striate units tuned to cardinal orientations (relative to oblique orientations) mentioned in the Introduction. That is, since there are more neurons tuned to horizontal, as more and more of those units are activated, their collective weight in the horizontal normalization pool is larger than the other three orientations, thereby decreasing sensitivity to broadband (i.e., spatial frequency and orientation) horizontal orientations. Additionally, the smaller increase of 
the inherent weights in the vertical weighting index can likewise be associated with the smaller bias (relative to the number of horizontally tuned neurons) of vertically tuned neurons in striate cortex.

\subsubsection{The Interactions Between the Two Inherent Weighting Indices}

In order to focus on the specific axes of the different inherent weighting indices' grids (as shown in Figures $\mathbf{4 0}$ and $\mathbf{4 1}$ ), the data along each of those grid lines were pulled out of those figures and plotted in Figure 42a and $\mathbf{4 2 b}$ in order to better view the interactions between the no-background and noise background experiments for each of the test orientations. Two graphs were constructed for each of the four test orientations, one that plots the change in the inherent weights as a function of increasing spatial frequency bandwidth of the increment extent (i.e., the variable spatial frequency graphs), and the other that plots the change in the inherent weights as a function of increasing orientation bandwidth of the increment extent (i.e., the variable orientation bandwidth graphs). Plotted within those graphs are the changes in the inherent weights from the nobackground indices (solid lines) and the noise background indices (dashed-lines). Each line thus represents the change in the inherent weights for while holding either spatial frequency or orientation bandwidth constant (represented by the different colored lines) and allowing the other variable vary (plotted on the abscissa). On the ordinate, of course, is the range of inherent weight values obtained from both of the no-background and noise background weighting indices. 
Judging from the graphs in the top portion of Figure 42a, the effect of the noise background conditions of the vertically oriented test increment weights was relatively minimal for the limited spatial frequency and orientations increment extents when the other parameter was allowed to vary. However, for the $20^{\circ}$ and $45^{\circ}$ constant spatial orientation bandwidth increment extents where spatial frequency was allowed to vary, the weights show a relatively high increase at the 1.0 octave and broadband spatial frequency bandwidth increment extents compared to the corresponding no-background conditions. Likewise, the vertical weights are much elevated across the entire range of orientation bandwidths for each of the 1.0 octave and broadband constant spatial frequency bandwidth increment extents. Here the discussion is returned to the idea of the bias in the number of neurons tuned to vertical orientations accounting for the weights in the noise background condition, but not in the no-background condition. As mentioned earlier, while a numbers bias has been observed for striate neurons that prefer vertical orientations, this bias may not be strong enough to reveal itself in the no-background condition; which might be possible if the biased number of vertically tuned cells are more tuned for orientations slightly off vertical. Thus, when the vertical increments are made to include a large range of orientations and spatial frequencies under broadband activation, the biased number of vertically and slightly off vertically tuned neurons would be more activated, thereby effectively contributing higher inherent weights to the normalization pool associated with processing vertical orientations.

For the graphs in the bottom portion of Figure 42a, the effect of the noise background conditions of the horizontally oriented test increment weights was rather extensive across 
all of the constant spatial frequency and orientations increment extents when the other parameter was allowed to vary. When the orientation bandwidth of the increment extent was held constant with variable spatial frequency increments, the single and $5^{\circ}$ orientation bandwidth conditions show rough monotonic increases while the other two orientation bandwidths show non-monotonic functions with increasing spatial frequencies for the no-background conditions. However, for the noise background conditions, the functions start out exhibiting small monotonic increases at the limited filter extent range, and become more pronounced as spatial frequencies are added to the increment extent. The non-monotonic functions for the no-background conditions depict a region where the weights are minimal, suggesting optimal suprathreshold sensitivity for horizontal orientations in the 0.5 to 1.0 octave range. This range of 'facilitation' is absent in the noise background conditions. Again, if one considers that the effect of the broadband noise pattern is to induce more overall activation, then this increased activation in the biased population of horizontally tuned units would serve to contribute higher weighting values to the normalization pool associated with processing horizontal orientations. For the conditions where the spatial frequency bandwidth of the increment extent is held constant and the orientation bandwidth of the increment extent is allowed to vary, the overall pattern of changes in the inherent weights are very similar between the nobackground and noise background conditions, but, in general, the overall magnitude of those functions is very different. Specifically, for the single frequency-variable orientation functions, the no-background conditions produce decreasing weighting functions while the noise background conditions show increasing functions. At this scale, the results can be attributed to the overall greater activation of the biased horizontal 
units in the noise background conditions. The magnitude shifts in the constant 0.5 and 1.0 octave ranges for the noise background conditions are very minimal, but again can be explained by the greater activation of the biased number of horizontal neurons. In the constant broadband spatial frequency conditions however, the no-background function starts out much more elevated than the noise background function until the $45^{\circ}$ bandwidth condition. It may be the case that the overall magnitude shift observed for the no-background broadband frequency function is modulated by a similar global gain control mechanism mentioned earlier for the oblique increment weighting functions.

Finally, since the upper and lower graphs in Figure $\mathbf{4 2 b}$ are relatively similar, the interactions between the weighting functions for the obliquely oriented test increments in the no-background and noise background conditions will be discussed simultaneously. In the conditions where the orientation bandwidths of the increment were held constant, the strongest interactions can be observed in the conditions where the orientation bandwidths of the increment extents were held at $5^{\circ}, 20^{\circ}$, and $45^{\circ}$ across increasing spatial frequency bandwidth of the increment extent. In those conditions, the effect of the noise background appears to be to increase the inherent weights on the normalization pools associated with processing oblique orientations. This effect can be explained by considering that the higher levels of activation in the noise background condition activated more obliquely tuned neurons as more and more spatial frequencies were included in the increment extent, thereby increasing the weights associated with those normalization pools. However, this pattern of interaction is absent in the conditions where the spatial frequency extent of the increment was held constant and the orientation 
bandwidth of the increment extent was allowed to vary. With the exception of the constant 1.0 octave spatial frequency bandwidth, the effect of the noise background appears to be a reduction in the inherent weights associated with the oblique orientation processing normalization pool. This suggests that the extent of the pooling for the oblique normalization pools is much more extended along the spatial frequency dimension.

\subsection{The Content-Dependent Processing Bias Weighting Indices}

The primary reason for carrying out the neural response simulations was to provide insight into how the neural response interaction weight (i.e., $w_{i j}$ ) of the proposed striate normalization model might change as a function of different types of natural scene content bias contained in different sets of imagery. The fundamental assumption in the current simulation was that local responses of the different linear filters utilized in the three different simulations resembled those of human striate cortex. By using the joint histograms of the different linear basis filter responses, it could be determined which filters were maximally responding to the same content as other filters. The idea here was that the more a given filter's responses (the primary filter) were to other filters (the secondary filters) that were tuned to other orientations and spatial scales, the stronger the weight of $w_{i j}$ which would in turn serve to reduce the response of the primary filter $\left(L_{i}\right.$ in the proposed model). Thus, if a given scene contained a large amount of vertical natural scene content, all neural units tuned to vertical or off-vertical orientations would respond strongly, thereby contributing a stronger weight to the normalization pool for the vertically tuned units - - effectively driving sensitivity down for that orientation. 
The neural response simulations carried out for the content-dependent effect 1 natural scene image set provided a generalized weighting index for imagery possessing a static content bias at any orientation (refer to Figure 37 for further details). This system of weights can be plugged into the proposed striate normalization model in order to effectively describe the pattern of results from the psychophysical experiments described in Section 2.2.2.1. However, since the weights are relatively shallow regression line slopes, a constant value (i.e., 1.0) will be added to each of the weights in order to ensure that the weights will actually increase the strength of the weighting pool. The neural response simulations carried out for the content-dependent effect 2 natural scene image set also provided a practical weighting index that can be applied to each orientation in the proposed striate normalization model since it was overall orientation sensitivity that was reduced for imagery with very shallow or very steep amplitude spectrum slopes (refer to Section 2.2.2.2 for further details). Specifically, for the images in the 0.659 slope set (i.e., the shallowest slope set), the magnitude of the averaged neural response dependencies were highest at the highest spatial scales (these were actually the highest values obtained across all image types). For the images in the steeper slope sets (i.e., 1.046 and 1.443 ), the magnitude of the averaged neural response dependencies are highest at the lowest spatial scales, which were the second and third highest values obtained across all image types. Finally, for the images in the slope sets between the extremes (i.e., $0.725,0.854$, and 0.949 ), the magnitude of the averaged neural response dependencies were, overall, the lowest. For this weighting index, the regression line slopes were also relatively shallow, however, a constant will not need to be added here. 
The reasoning is that since the effect of the different natural scene image slopes was to reduce the over all sensitivity in the shallow and steep amplitude spectrum slope ranges, the larger weighting values (close to, but not exceeding 1.0) will actually allow for the full expression of whatever inherent weights are being applied in the different gain pools. The effect of the smaller weights obtained from the images with amplitude slopes typically found in natural scenes will be to reduce whatever inherent weights are present in the different weighting pools, thereby increasing overall sensitivity. The weighting system obtained from the neural response simulations carried out for the contentdependent effect 3 natural scene image set provided a generalized weighting index for imagery possessing a variable content bias at the horizontal orientation. Recall that the pattern of results obtained from the psychophysical experiments described in Section 2.2.2.3 showed that overall orientation sensitivity increased with increasing amounts of horizontal content. The results from the neural response simulations carried out with the images used in that experiment yielded increasing filter response dependencies across the limited set of images; with that increase being proportional to the amount of horizontal content contained in those images. Thus, in order to properly model the psychophysical results described in Section 2.2.2.3, the weights must be implemented by dividing the normalization pool of each orientation by the weight associated with a given amount of horizontal content in a natural scene, effectively increasing sensitivity to all orientations. The primary reason for this weight application is to effectively model the data (i.e., the results described in Section 2.2.2.3) and can be reasonably supported by considering the premise that there exist a greater number of units tuned to horizontal orientations in striate cortex. Specifically, as the amount of horizontal content increases across a series 
of natural scenes, the activity of units specifically tuned to horizontal orientations is reduced (a result of the content-dependent effect 1). Thus, any inhibitory effects the biased number of horizontal units had on the sensitivity of off-horizontally tuned would be released, effectively increasing their sensitivity to the oriented content in which they are tuned. However, sensitivity for detecting horizontal increments in the experiments described in Section 2.2.2.3 also increased with the relative amount of horizontal content. Given that response dependencies have been observed between units tuned to horizontal and units tuned to either $45^{\circ}$ and $135^{\circ}$ oblique (i.e., Wainwright et al. 2001), such an improvement in horizontal sensitivity can potentially by attributed to more vigorous activity of the obliquely tuned units to horizontal content.

\subsection{A Schematic Representation of the Proposed Striate Normalization Model}

In the Wainwright et al. (2001) gain control model, the dynamic weights (i.e., $w_{i j}$ ) that were implemented into the normalization pool were based on observations of the strength of the conditional probabilities of simulated neural responses for any given portion of a any given natural scene (Simoncelli, 1999; Wainwright et al., 2001; Schwartz \& Simoncelli, 2001). However, the extent to which the activity of a given primary filter was locally similar to that of other basis filters, tuned orientations and spatial scales different from that of the primary filter, was not made explicit. The results from the neural response simulation plotted in Figure 37 on the other hand show, for the bank of basis filters used in the current study (which was very extensive), which filters most often responded to the same locations of a natural scene content bias at one particular orientation. Thus, in relation to a given primary basis filter, Figure 37 shows the region 
in the basis filter response space where filters are responding to the same (or similar) content in a natural scene with a content bias at a given orientation (the dark to light red region). While such a region was not made explicit by Wainwright and colleagues (e.g., Simoncelli, 1999; Schwartz \& Simoncelli, 2001), it is precisely the extent of that region that forms the normalization pool for the neural units 'contained' in that region (i.e., those responding to the biased content in a given natural scene). Note that the spread of that region is very much broadband, and while there is an elevated region of response dependency along the orientation dimension (near to the Scale 2 axis), it is the oval $\sim .90$ to $\sim .60$ weighting region that will be the focus for defining the extent of the normalization pool for the proposed striate normalization model.

In order to provide a meaningful illustration of the extent of the normalization pool with respect to the bank of basis functions used in the neural response simulations (of which represents a sample of neurons in striate cortex), the results plotted in Figure 37 where superimposed over the basis filter function bank as shown in Figure 43. For that figure, consider each level in turn. First, part (a) simply serves as a representative natural scene image that contains a content bias at a given orientation $\left(45^{\circ}\right.$ in that figure), and is the input image to the bank of filters shown in part (b). For that example, the bank of filter functions serves as a representative sample of neurons in a given region in striate cortex sampling content in the image at a range of orientations and spatial scales. The arrows rising up from the filters (i.e., part 'c') represent the neural signal from each unit. Note that the arrows are grayscale coded with respect to the bias in the numbers of those units tuned to different orientations (refer to the caption for details). The dynamic weighting index obtained form the content-dependent effect 1 simulation has been shifted 
in order to align the region of high dependency over the units tuned to the same orientation as the content bias in the input image. Thus, part (d) provides a visual approximation regarding the extent of the normalization pools that will be associated with each set of neural units tuned to one of the four test orientations investigated in Experiments 1 and 2.

Having provided a visual example of the extent of the normalization pool that will be used for each of the four orientation responses to be explained by the proposed striate normalization model, the next step is to provide a generalized schematic of how the inherent weighting component works in the proposed model (see Figure 44). In order to represent the normalization pools practically for each of the four orientations of interest, 3D Gaussian profiles were used as an approximation. Note that Figure 44 is set up in the same fashion as Figure 43, but differs conceptually. The input images (a) are sample stimuli used in Experiment 2 at each one of the test orientations. The increment extent of the example stimuli was broadband spatial frequency and had a $45^{\circ}$ orientation bandwidth. Note that the primary interest of the proposed model lies in adjusting the responses of neural units that are contained within a given normalization pool. Accordingly, the schematic in this figure is structured to show how the output of a given range of neural units is adjusted when presented with visual structure at the orientation that best drives that range of units. Thus, each of the four input images are meant to be considered as only driving the columns of neural units indicated by the arrows. Again, parts (b) and (c) of Figure 44 show the representative sample of neurons in a given region in striate cortex sampling content in the stimuli at a range of orientations and 
spatial scales, and their grayscale coded biased output. Part (d) depicts the four approximated normalization pools, each receiving active input from a broad range of neural units (due to the large extent of the triangle filter that generated the input images). The broad arrow exiting the top of each normalization pool is representative of the output of each of the units contained within that normalization pool. Part (e) depicts each of the four weighting indices for the four orientations, as well as from which portion of those indices that the weight is drawn in order to scale each of the outputs in their respective normalization pools. Note that the schematic of the proposed striate normalization model depicted in Figure 44 is incomplete, that is, only the inherent component has been described. There still remains how the dynamic weighting components fit in to such a schematic rendition of the proposed model. It should be mentioned at this point that the schematic representation of the proposed striate model shown in Figure $\mathbf{4 5}$ is simply to provide a visual of how and where the different weighting indices fit into the proposed model. The example itself is therefore hypothetical because it is not known how the different weighting indices will change the total output of the system when simultaneously 'implemented' with the other indices. The input image in that figure (i.e., level ' $\mathbf{a}$ ') is a broadband natural scene image with a steep amplitude spectrum slope (1.6), a content bias at the $45^{\circ}$ oblique orientation ( $32 \%$ bias - refer to Section 7.6 .2 ofr details regarding how this percentage is calculated), and has been incremented with a broadband frequency- $-45^{\circ}$ orientation bandwidth triangle increment centered on each of the four orientations of interest. Even though the weighting indices depicted in Figure 45 were generated from neural response simulations that produced results that could account for the results of the psychophysical data described in Sections 2.2.2.1 - 2.2.2.3, they were 
not directly generated from psychophysical data, and thus it is unknown whether the weights for those indices correspond to the actual weights responsible for the sensitivity fluctuations in the psychophysical data reviewed in the above mentioned sections. Additionally, the input image in the Figure 45 schematic was chosen because it possessed all of the physical properties that would, theoretically, cause input to be provided to the normalization pool from all of the indices generated in the current study. Moving up the figure, parts (b) and (c) show the representative sample of neurons in a given region in striate cortex sampling content in the stimuli at a range of orientations and spatial scales, and their grayscale coded biased output. However, as indicated in part (d), the grayscale shaded output arrows from the representative striate units are assigned a color based on the weighting index (note the coloration of the column outlined in black - the 3D map is the same as that shown in Figure 38) from the content-dependent effect 2 simulation. Since the input image has a very steep slope, the units tuned to lower spatial frequencies across all orientations are weighted more than those optimally tuned for higher spatial frequencies. The next weighting index represented is the dynamic index (shown far left-top) that was generated from the content-dependent effect 1 simulation (see also Figure 43). As mentioned earlier, the region of that index outlined in black represents the strength and extent of the normalization pool associated with each of the four orientations. The color space of that weighting strength has been mapped onto the Gaussian representations of the normalization pools indicating which units will receive the strongest weights within that pool. Note that the content-dependent effect 3 simulation weighting index is not present in this figure. However, the implementation of that weight would simply act to scale the weights mapped onto the Gaussian pools. Part 
(e) of the full schematic of the proposed striate normalization model operates in the identical manner described in Figure 44, only here, the noise background weighting indices are represented given the fact that the input image has energy at all spatial frequencies and orientations. The final output of this system for the input image would be similar to the far right portion of the graph shown in Figure 20, only the $45^{\circ}$ bar would be much more reduced due to the presence of the bias in content at that orientation. Again, the full schematic of the proposed model is not meant to be taken literally, rather it serves as a representation of how all of the weighting indices, obtained in the current study, would be simultaneously implemented. Should the design and assumptions of the proposed model hold true, the model should be able to predict the results from psychophysical experiments that utilize natural scene imagery similar to the input image shown in Figure 45. Accordingly, future experiments designed to test human sensitivity to triangle increments applied to natural scenes with a fixed content bias a given orientation and variable amplitude spectrum slopes would prove beneficial in testing the accuracy of the proposed model's predictions. 


\section{GENERAL CONCLUSIONS}

In the current study, a number of questions were investigated, all of which were centered on how the human visual system processes orientation in broadband stimuli. Recently, Wainwright et al. (2001) proposed a functional model in order to describe how the human visual system might carry out the task of contrast normalization for naturalistic stimuli. This model was different from the earlier cortical gain control models in that it involved selective normalization of the output of striate neurons that are tuned to different orientations in real-world stimuli. The Wainwright model is thus optimally suited to weight the output of a range of 'neural units' selective for a given orientation more (relative to neural units tuned to other orientations) when a given broadband stimulus contained a bias in the amount of structure at that orientation. In fact, Hansen et al. (2003) successfully demonstrated that this model could account for the content-dependent effect 1, described earlier in Section 2.2.2.1, with a relatively small number of content biased natural scene imagery. The results from the neural response simulations described in Section 7.6.1 were carried out with a much larger content biased image set and provided further evidence that the Wainwright model could account for the contentdependent effect 1 . The results of those simulations also showed that the highest dynamic weights for a given neural unit arose from other neural units more selective for spatial frequencies approximately one octave below and one octave above that unit's 
preferred spatial frequency. Along the orientation dimension, the higher weights were contributed by the neural units selective for orientations approximately $\pm 30^{\circ}$ from the preferred orientation of the given neural unit. The results from the neural response simulations reported in Section 7.6.2 provided another dimension to the way in which the Wainwright model could be used to describe how striate cortex implements contrast normalization. Specifically, those results demonstrated that the magnitude of the 'simultaneous responses' between neural units that were tuned to similar orientations and spatial frequencies changed as a function of the slope of the amplitude spectrum of natural scenes that did not contain any biases in content at different orientations. In fact, the second neural response simulation carried out in the current study showed that such neural unit responses were at a minimum when the slope of the amplitude spectrum, of the experimental imagery, was close to that most often observed in typical natural scenes. It was only when the amplitude slopes were very shallow or very steep when the magnitudes of the response co-occurrences were observed to be at their highest. Thus, by incorporating the weights derived from the simulated neural responses from the second simulation into the Wainwright model, the psychophysical results described in Section 2.2.2.2 can be successfully accounted for. Note that it would be only the overall decrease in sensitivity observed across all of the tested orientations that would be accounted for by implementing these weights into the Wainwright model. The results from the neural response simulations reported in Section 7.6.3 added yet another dimension to the way in which the Wainwright model could be used to describe how striate cortex implements contrast normalization. What those neural response simulations demonstrated was the magnitude of the neural response co-occurrences increased dramatically across a set of 
natural scene imagery that exhibited a general increase in horizontal content from one image to the next. Based on the assumptions described in Section 8.3, the Wainwright model can successfully account for the psychophysical results described in Section 2.2.2.3 when the weights derived from the simulated neural responses are implemented in an inhibitory manner.

While the Wainwright et al. (2001) model could be updated with different weighting indices based on the content biases of different types of natural scene imagery, such a model does not take into account the inherent horizontal effect bias found to occur in all of the experiments reviewed in the sections devoted to human visual processing of orientation in the context of broadband stimuli, as well as demonstrated in Experiments 1 and 2 of the current study. Since the general horizontal effect has been demonstrated to occur with stimuli consisting of natural scenes as well as with broadband visual noise stimuli (Essock et al., 2003; Hansen \& Essock, 2003; 2004a), it seems reasonable to expect that it could be due to a static anisotropy inherent in the normalization pool (i.e., an inherent bias). Such a component would most likely arise directly from the greater prevalence of neurons with a horizontal preferred orientation contributing more heavily to the pooled response. The existence of this numerical bias (a horizontal effect of orientation preferences) has been clearly documented by a large number of electro/neurophysiologists (e.g. Li, Peterson, \& Freeman, 2003; Tiao \& Blakemore, 1976; Chapman, Stryker, \& Bonhoeffer, 1996 (easily seen in their Figures 1 and 2); Chapman \& Bonhoeffer, 1998 (easily seen in their Figures 1 and 2); Coppola, White, Fitzpatrick, \& Purves, 1998; Yu \& Shou, 2000; Mansfield, 1974; Mansfield \& Ronner, 1978). Thus, it 
was argued in Section 4.0 that an inherent weighting factor needed to be added to the Wainwright model (as well as all other normalization models) such that the divisive pool is influenced by both the dynamic weighting factors described earlier, as well as an inherent anisotropic weighting factor. However, the proposed striate normalization model (i.e., the Wainwright model with the inherent weighting component included) simply suggests plugging an extra variable into the normalization pool for each range of orientation selective neural units originally laid out by Wainwright et al. (2001). That is, one is left with too many unknowns regarding how the proposed striate normalization model could also account for the horizontal effect. Specifically, if it is indeed the bias in the number of horizontally, and to a lesser extent vertically, tuned striate cells that causes the reduction of horizontal sensitivity to a broad spatial frequency/orientation amplitude increment, then one would expect that if the extent of the increment (in terms of total number of spatial frequencies and/or orientations incremented) is reduced, the presence of the horizontal effect should also diminish. Thus, Experiments 1 and 2 of the current study were carried out in order to show exactly how the inherent weights change in the normalization pools (i.e., the normalization pools associated with populations of neurons tuned to different orientations) of the proposed model as a function of the amount of activation within the different populations of striate neurons alone. This activation of course was generated by utilizing different triangle filter increment extents. Additionally, in order to show how the inherent weights (i.e., $o_{i j}$ ) might change within the neural populations tuned to different orientations as a function of increment extent when all neural units are activated, broadband $1 / f$ noise patterns were embedded with triangle increments of variable extent. Because the Class 1 oblique effect has been demonstrated 
with high spatial frequency gratings and the horizontal effect was demonstrated to occur with a broad range of spatial frequencies across a $45^{\circ}$ bandwidth of orientations, the increment extents utilized in both experiments were made to start out as a single point in the Fourier domain (i.e., a sinusoidal grating in the spatial domain) and gradually expand along the radius (toward the DC) and theta coordinates in the Fourier domain (i.e., analogous to adding/incrementing more and more sinusoidal gratings of differing spatial frequencies and orientations in the spatial domain). Thus it was also the intent of both Experiments 1 and 2 to show the transition point between the oblique effect and the horizontal effect as the extent of the triangle increment increased, as well as whether the shift occurred earlier when the extent of the increment was increased along one dimension (i.e., spatial frequency or orientation bandwidth) while holding the extent of the increment constant along the other dimension. In the context of the proposed striate normalization model, the variable increment extent paradigm allowed for insight into along which dimension the neural unit numbers bias is strongest as well as how, in general, the different populations of neurons tuned to different orientations interact within their respective normalization pools.

The results from the two psychophysical experiments carried out in the current study demonstrate a gradual shift from the oblique effect, in conditions where the increment extent was relatively narrow along either the spatial frequency or orientation dimension and allowed to vary along the other dimension, to the horizontal effect in conditions where the increment extent was rather broad along both dimensions. Relatively speaking, when the increment extent contained moderate amounts of spatial frequencies and 
orientations, very little, if any, suprathreshold orientation anisotropy was observed. For both experiments, this range was approximately in the 0.5 to 1.0 octave and $5^{\circ}$ to $20^{\circ}$ orientation bandwidth range of increment extent. This suggests that what ever interactions that are taking place in the normalization pools associated with processing specific ranges of orientations is resulting in an overall equilibrium or null point at which the perceived strength of all of the orientations tested is equal. The effect of the noise background was very minimal with respect to shifting the 'nullifying' conditions closer to or further from the conditions where a definite oblique effect or horizontal effect was exhibited. However, with respect to changing suprathreshold sensitivity for each of the four orientations across the different increment extent conditions and between corresponding conditions from the two experiments, the noise background had a somewhat generalized effect of decreasing suprathreshold sensitivity in a number of the conditions examined in the current experiment.

In the interest of brevity, the conclusions that will be drawn from the observed changes in suprathreshold sensitivity for each of the test orientations as a function of increment extent, as well as the changes of those functions observed in the presence or absence of a $1 / f$ noise background, will be made in turn for each of the cardinal orientations test orientations. However, given the relative similarity between the two oblique weighting indices, both will be described together. Based on the weighting functions plotted in Figure $\mathbf{4 2 b}$, the most general feature that stands out in the nobackground functions where orientation was held constant and spatial frequency was allowed to vary is the shift from an increasing monotonic function (for the single 
orientation conditions) to increasingly stronger non-monotonic functions as the spatial frequency bandwidth increases (across the broader orientation bandwidths). This pattern of weight changes could possibly be explained by an increase in the summation of neural units responding to those orientations as more and more spatial frequencies are included in the test increment. The facilitory region of the non-monotonic functions suggest that the effect of pooling increasing numbers of obliquely oriented striate units is inhibitory in nature, where each individual unit is normalized by a strongly inhibited normalization pool. In fact, a fairly reasonable argument could be made that the overall magnitude of the changing functions is gradually decreasing with increasing spatial frequency bandwidth, implying that, across increasing spatial frequency bandwidths of the increment, the pattern of changes within each of the functions is modulated by a 'local' gain control, while the overall magnitude of the functions is modulated by some 'global' gain control mechanism. In the corresponding coordinates of the noise background condition, the narrower orientation bandwidth conditions exhibit more of a quadratic increase, which can still be explained by increased summation in the normalization pool which turns slightly inhibitory in the broadband spatial frequency condition. Additionally, instead of exhibiting a facilitory region in the two broader orientation increment extent conditions with increasing spatial frequencies, the weights tend to level off. When considering this pattern of changes between the background and nobackground conditions in the context of the differential neural numbers biases discussed earlier, the argument can be made that the additional activity caused by the noise pattern obscures that region due to increases activity. Judging from the no-background functions where spatial frequency was held constant and orientation bandwidth was allowed to 
vary, the most obvious trend is the shift from an increasing monotonic function (for the single spatial frequency conditions) to increasingly stronger decreasing monotonic functions as orientation bandwidth increases (across the broader spatial frequency bandwidths), as well as the shift in the magnitude of the broadband spatial frequency function. The increasing monotonic function can be accounted for by the same explanation offered for that in the constant orientation graph. The decreasing region of the monotonic functions suggest that the effect of pooling increasing numbers of obliquely oriented striate units is inhibitory in nature, where each individual unit is normalized by a strongly inhibited normalization pool (note that those functions remain 'inhibited', whereas in the constant orientation bandwidth extent conditions, the functions begin to rise again). However, while the broadband frequency function decreases monotonically, it is dramatically elevated relative to the other two decreasing monotonic functions suggesting an additional gain modulation is being applied along the orientation dimension for broadband frequency increment extents. A similar pattern of function changes can still be observed in the corresponding coordinates of the noise background functions. The similarity is a general trend in the functions toward exhibiting rough decreasing monotonic functions where overall magnitude of the functions peaks for constant 1.0 octave bandwidth increment instead of in the constant broadband frequency increment. However, different from the constant orientation bandwidth function graphs, a fairly reasonable argument could be made that the overall magnitude of the changing functions is gradually decreasing with increasing orientation bandwidth, again, implying that across increasing orientation bandwidths of the increment, the pattern of changes 
within each of the functions is modulated by a local gain control, while the overall magnitude of the functions is modulated by some global gain control mechanism.

Moving on to the weighting functions plotted in Figure 42a, if the peaks in the functions for the vertical test increment at the single orientation- $20^{\circ}$ increment bandwidth is treated as an anomaly (refer to Section 8.2.1.1 for an explanation), there is only very minimal variation in all of the vertical functions for the no-background conditions. However, the corresponding functions in the noise background condition exhibit increasing weights when either the spatial frequency or orientation bandwidth of the increment extent was made very broad. As mentioned in Section 8.2.2, while a numbers bias has been observed for striate neurons that prefer vertical orientations, this bias may not be strong enough to reveal itself in the no-background condition; which might be possible if the biased number of vertically tuned cells are more tuned for orientations slightly off vertical. Thus, when the vertical increments are made to include a large range of orientations and spatial frequencies under broadband activation, the biased number of vertically and slightly off vertically tuned neurons would be more activated, thereby effectively contributing higher inherent weights to the normalization pool associated with processing vertical orientations. For the horizontal increments, when the orientation bandwidth of the increment extent was held constant with variable spatial frequency increments, the narrower orientation bandwidth conditions show rough monotonic increases while the other two orientation bandwidths show non-monotonic functions with increasing spatial frequencies for the no-background conditions. However, for the noise background conditions, the functions start out exhibiting small monotonic increases at the 
limited filter extent range, and become more pronounced as spatial frequencies are added to the increment extent. As mentioned in Section 8.2.2, the non-monotonic functions for the no-background conditions depict a region where the weights are minimal, suggesting optimal suprathreshold sensitivity for horizontal orientations in the 0.5 to 1.0 octave range. This range of 'facilitation' is absent in the noise background conditions. If the effect of the broadband noise pattern is to induce more overall activation, then this increased activation in the biased population of horizontally tuned units would serve to contribute higher weighting values to the normalization pool associated with processing horizontal orientations. For the conditions where the spatial frequency bandwidth of the increment extent is held constant and the orientation bandwidth of the increment extent is allowed to vary, the overall pattern of changes in the inherent weights are very similar between the no-background and noise background conditions. However it should be noted that the overall magnitude of those functions is very different. Specifically, for the single frequency-variable orientation functions, the no-background conditions produce decreasing weighting functions while the noise background conditions show increasing functions. At this scale, the results can be attributed to the overall greater activation of the biased horizontal units in the noise background conditions. The magnitude shifts in the constant 0.5 and 1.0 octave ranges for the noise background conditions are very minimal, but again can be explained by the greater activation of the biased number of horizontal neurons. In the constant broadband spatial frequency conditions however, the no-background function starts out much more elevated than the noise background function until the $45^{\circ}$ bandwidth condition. It may be the case that the overall magnitude shift observed for the no-background broadband frequency function is modulated by a 
similar global gain control mechanism mentioned earlier for the oblique increment weighting functions. On a final note, the fact that rapid increases for the no-background weighting functions are observed in the constant orientation conditions where spatial frequency was allowed to vary is not observed in the conditions where spatial frequency was held constant provides further support to the suggestion made in Section 8.1.1, in that the inherent bias for the horizontally tuned bias is distributed more heavily along the spatial frequency dimension then along the orientation dimension.

Even the most general of overviews of the weighting indices generated from the data of Experiments 1 and 2 demonstrates that the interactions among striates neurons is remarkably complex. Despite this complexity, a few general trends stand out in the inherent weighting indices. The first being the overall similarity among the inherent weighting functions for both of the obliquely oriented test increments. In the context of the proposed striate normalization model, this similarity suggests that the nature in which the neural units are pooled in those normalization pools is very similar. However, in the no-background weighting indices, the nature in which the neural units tuned to vertical are pooled differs very much from the nature in which the horizontal units are pooled. However, in the context of a broadband noise background, the inherent weighting indices for those two orientations are much more similar, with the vertical index exhibiting an overall reduction in the magnitude of the inherent weights. Considering the neural numerical bias, the discrepancy between the inherent weighting indices from the two experiments can possibly be explained by the fact that the activity evoked by the noise background drives more of the vertical neural bias, thereby causing a general increase in 
the inherent weights as the extent of the triangle increment is increased. The implication here is that the bias in vertically tuned units is more evenly distributed across both the spatial frequency and orientation tuning dimension. The latter implication is contrasted with the general differences observed for the horizontally tuned units which exhibited an increase in their inherent weights along the spatial frequency dimension for the constant single orientation condition in the no-background experiment. This suggests that the bulk of the neural numbers bias for that orientation is contained more along the spatial frequency dimension. With respect to the broadband noise background activation experiment, the most general effect appeared to be to contribute more input into the inherent weighting pools for each of the four test orientations. Additionally, the presence of the noise background had little effect on the overall shape pattern of effects (oblique effect or horizontal effect pattern of results), with the one exception of decreasing suprathreshold sensitivity for broad extent vertical increments already discussed. Across both experiments and especially in Experiment 2, for all four of the test orientations ${ }^{4}$, there appeared to be two forms of gain modulation of the inherent weights. The first appeared to modulate the weights locally as a function of the increasing extent of the varied parameter. The other appeared to operate more globally by modulating the overall magnitude of the inherent weighting functions themselves as a function of increasing extent of the constant parameter. The local modulation component as already been extensively commented on, however, the nature of the global modulation has yet to be made explicit. The global modulation of the overall magnitude of the functions was

\footnotetext{
${ }^{4}$ The extent in which the vertical test increments exhibited both of these gain modulations was not relatively clear, and hence inconclusive. It may, however, be the case that the psychophysical paradigm employed in the current experiments was not ideally suited to demonstrate the two forms of modulation for the vertical test increments.
} 
most clear for both of the oblique test increments and exhibited a decreasing function with increasing bandwidth of the constant parameter, when the constant parameter was orientation bandwidth increment extent. When the constant parameter was spatial frequency bandwidth, the global modulation exhibited an increasing function of the overall magnitudes of the inherent weighting functions. For the horizontal test increments however, the global modulation was always increasing the overall magnitude of the inherent weighting function regardless of whether or not the constant parameter was spatial frequency or orientation bandwidth. Thus the latter global modulation may simply reflect the influence of the neural numbers bias of the horizontally tuned neurons. On a related note, across both experiments, the nature in which the weighting index functions changed when either spatial frequency or orientation bandwidth of the increment were held constant while allowing the other parameter to vary was dramatically different. This suggests, with respect to the proposed striate normalization model, that the two parameters are not equivalent with respect to their relative impact on the different normalization pools. Specifically, the effect of increasing the extent of the triangle increment on the different normalization pools depended on whether or not the dimension along which the expansion was occurring was spatial frequency or orientation (local modulation) as well as whether or not the constant parameter was spatial frequency or orientation (global modulation). 


\section{Table 1}

Triangle Increment Extent Conditions for Experiments 1 and 2

\begin{tabular}{|c|c|c|c|}
\hline $\begin{array}{c}\text { Condition 13: } \\
\text { Spatial Frequency: } \\
\text { Broadband: } 0.2-16 \mathrm{cpd} \\
\text { One Orientation }\end{array}$ & $\begin{array}{c}\text { Condition 14: } \\
\text { Spatial Frequency: } \\
\text { Broadband: } 0.2-16 \mathrm{cpd} \\
\text { Orientation Bandwidth: } \\
5^{\circ}\end{array}$ & $\begin{array}{c}\text { Condition 15: } \\
\text { Spatial Frequency: } \\
\text { Broadband: } 0.2-16 \mathrm{cpd} \\
\text { Orientation Bandwidth: } \\
20^{\circ}\end{array}$ & $\begin{array}{c}\text { Condition 16: } \\
\text { Spatial Frequency: } \\
\text { Broadband: } 0.2-16 \text { cpd } \\
\text { Orientation Bandwidth: } \\
45^{\circ}\end{array}$ \\
\hline $\begin{array}{c}\text { Condition 9: } \\
\text { Spatial Frequency: } \\
1 \text { Octave: } 8-16 \mathrm{cpd} \\
\text { One Orientation }\end{array}$ & $\begin{array}{c}\text { Condition 10: } \\
\text { Spatial Frequency: } \\
\text { 1 Octave: } 12-16 \mathrm{cpd} \\
\text { Orientation Bandwidth: } \\
5^{\circ} \\
\end{array}$ & $\begin{array}{c}\text { Condition 11: } \\
\text { Spatial Frequency: } \\
\text { 1 Octave: } 12-16 \text { cpd } \\
\text { Orientation Bandwidth: } \\
20^{\circ} \\
\end{array}$ & $\begin{array}{c}\text { Condition 12: } \\
\text { Spatial Frequency: } \\
1 \text { Octave: } 12-16 \mathrm{cpd} \\
\text { Orientation Bandwidth: } \\
45^{\circ} \\
\end{array}$ \\
\hline $\begin{array}{l}\text { Condition 5: } \\
\text { Spatial Frequency: } \\
1 / 2 \text { Octave: } 12-16 \mathrm{cpd} \\
\text { One Orientation }\end{array}$ & $\begin{array}{c}\text { Condition 6: } \\
\text { Spatial Frequency: } \\
1 / 2 \text { Octave: } 12-16 \mathrm{cpd} \\
\text { Orientation Bandwidth: } \\
5^{\circ}\end{array}$ & $\begin{array}{c}\text { Condition } 7: \\
\text { Spatial Frequency: } \\
1 / 2 \text { Octave: } 12-16 \mathrm{cpd} \\
\text { Orientation Bandwidth: } \\
20^{\circ}\end{array}$ & $\begin{array}{c}\text { Condition 8: } \\
\text { Spatial Frequency: } \\
1 / 2 \text { Octave: } 12-16 \text { cpd } \\
\text { Orientation Bandwidth: } \\
45^{\circ}\end{array}$ \\
\hline $\begin{array}{c}\text { Condition 1: } \\
\text { One Spatial Frequency: } \\
16 \mathrm{cpd} \\
\text { One Orientation }\end{array}$ & $\begin{array}{c}\text { Condition 2: } \\
\text { One Spatial Frequency: } \\
16 \mathrm{cpd} \\
\text { Orientation Bandwidth: } \\
5^{\circ}\end{array}$ & $\begin{array}{c}\text { Condition 3: } \\
\text { One Spatial Frequency: } \\
16 \mathrm{cpd} \\
\text { Orientation Bandwidth: } \\
20^{\circ}\end{array}$ & $\begin{array}{c}\text { Condition 4: } \\
\text { One Spatial Frequency: } \\
16 \text { cpd } \\
\text { Orientation Bandwidth: } \\
45^{\circ}\end{array}$ \\
\hline
\end{tabular}



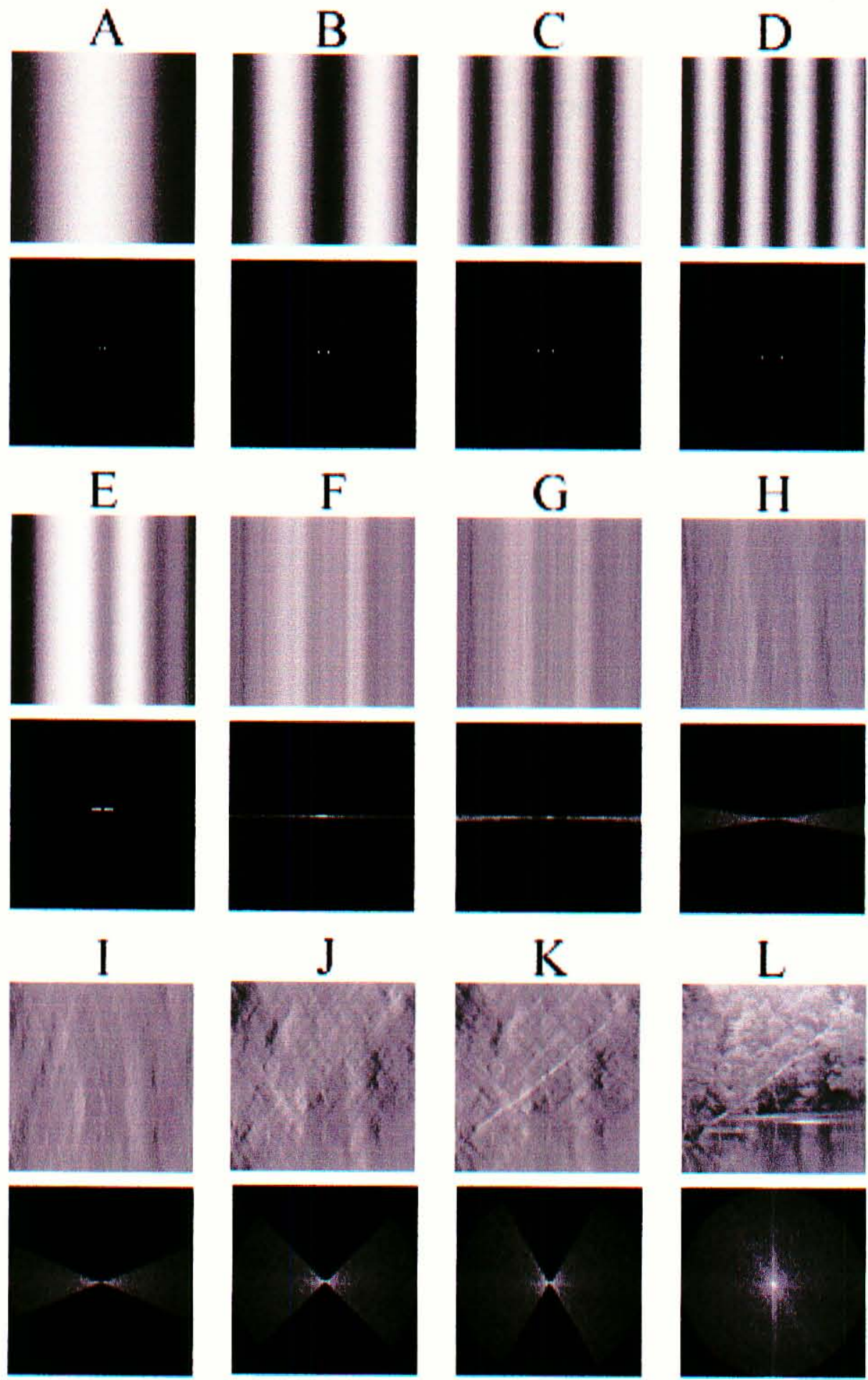

Figure 1. An example of the building up of a complex 2D signal (i.e., luminance defined image) utilizing sinusoidal waves consisting of different amplitudes, spatial frequencies, phases, and orientations via the Inverse Fourier transform. For each row, the bottom 
figures are in the frequency/Fourier domain and figures directly above are their representation in the spatial domain. A-D) examples of different vertical sinusoidal waveforms increasing in spatial frequency (note that in the spatial domain the waveforms are global and in the Fourier domain are localized at one particular point. E) The sum of the spatial frequencies depicted in A-D. F) the sum of all vertically oriented spatial frequencies up to the Nyquist limit. G) The sum of all spatial frequencies located within a $5^{\circ}$ wedge centered on vertical. H) The sum of all spatial frequencies located within a $20^{\circ}$ wedge centered on vertical. I) The sum of all spatial frequencies located within a $45^{\circ}$ wedge centered on vertical. $J$ ) The sum of all spatial frequencies located within a $90^{\circ}$ wedge centered on vertical. K) The sum of all spatial frequencies located within a $120^{\circ}$ wedge centered on vertical. L) The entire amplitude spectrum. 

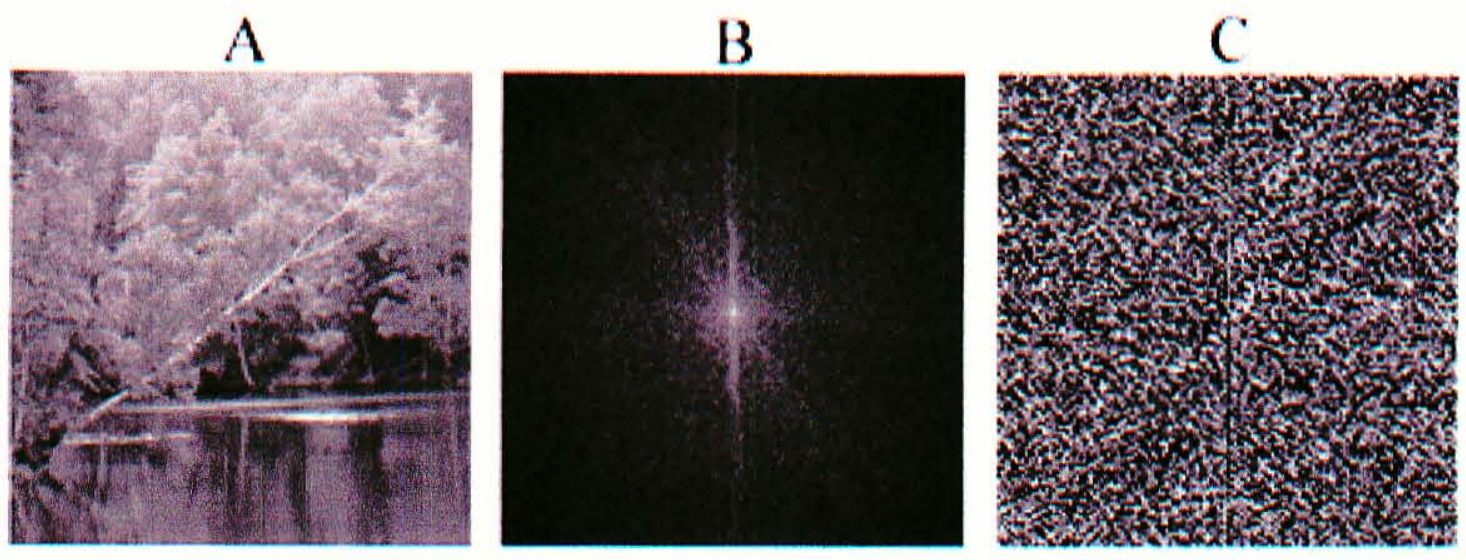

Figure 2. A) An example of a natural scene image. B) The corresponding 2D amplitude spectrum of the Fourier transformed image depicted in A. C) The corresponding phase spectrum of the image depicted in A, note that each point in this spectrum is coded with grayscale values $0-255$ for the range of values $-\pi$ to $\pi$; see Figure 3 and text for further information regarding the 2D amplitude spectrum. 

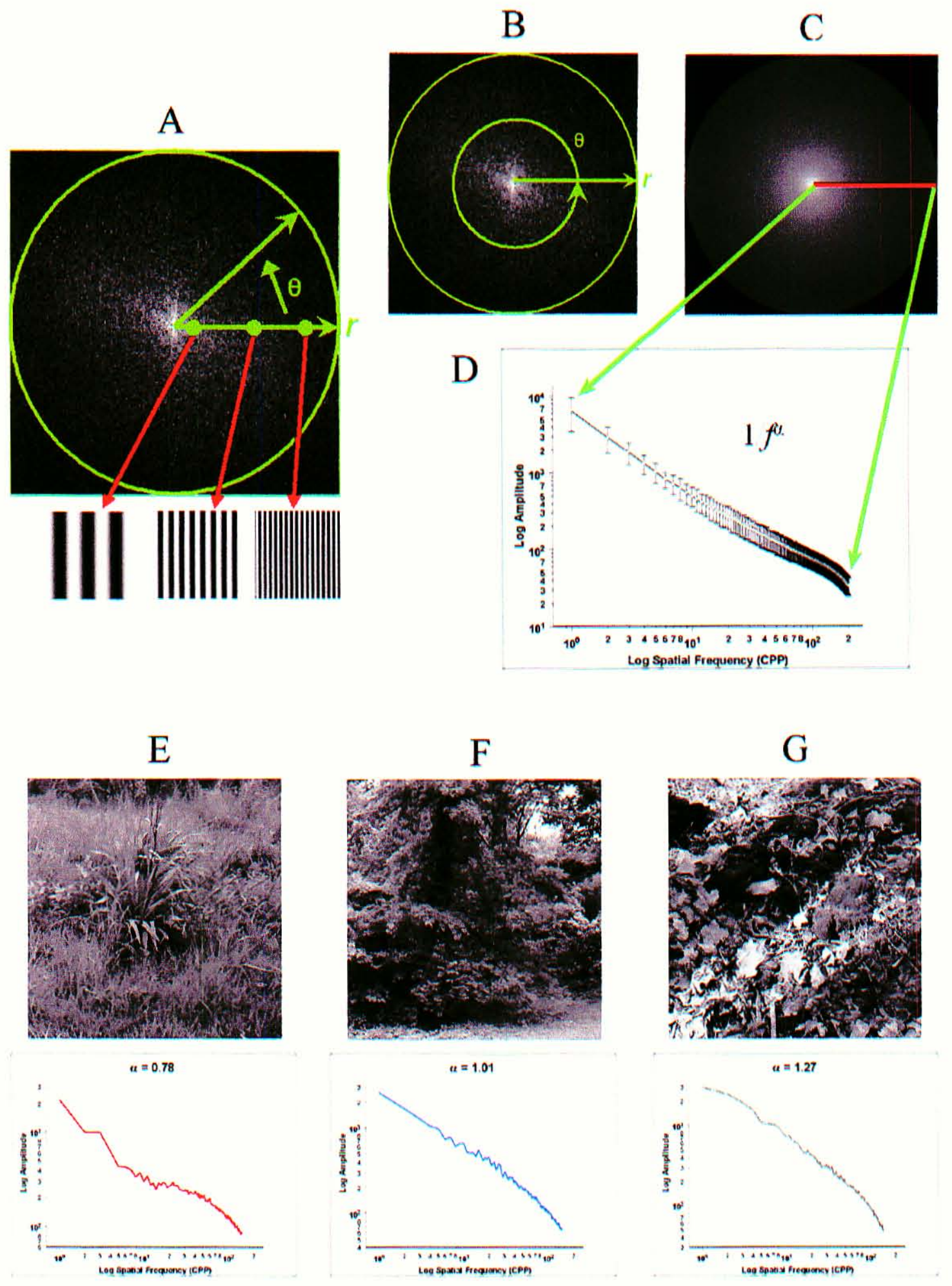

Figure 3. A) A depiction of an example amplitude spectrum. Note that the different amplitudes of the spatial frequencies are plotted in polar coordinates. The different 
spatial frequencies are plotted on the radius axis, with the spatial frequency increasing with increasing spatial frequency. The theta axis plots the different orientations, note that the orientation coordinate system is rotated $90^{\circ}$ such that spatial frequencies on the highlighted horizontal axis actually depicts vertical spatial frequencies in the spatial domain. In addition, the Fourier transform of a 2D complex signal is an odd-symmetric transform, where the top half of the spectrum is mirrored on the bottom half (i.e., the top and bottom halves of the spectrum are equivalent). B-D) An example of the process behind calculating the slope (i.e., $\alpha$ value) of the amplitude spectrum. B) The first step involves averaging each spatial frequency at each radius coordinate across all orientations. C) An example of the orientation amplitude spectrum where each point on the radius axes is replaced with the average value obtained in the step described in B. D) This figure shows the average fall-off of amplitude with increasing spatial frequency averaged across all orientations (taken from a random sample of 231 natural scene images) plotted on double logarithmic axes. Note that the values on this plot were obtained by averaging the highlighted vector shown in $\mathrm{C}$ from the set of randomly sampled images. E-G) Examples of different natural scene images (top) and their corresponding double logarithmic orientation averaged amplitude spectrum fall-offs. 


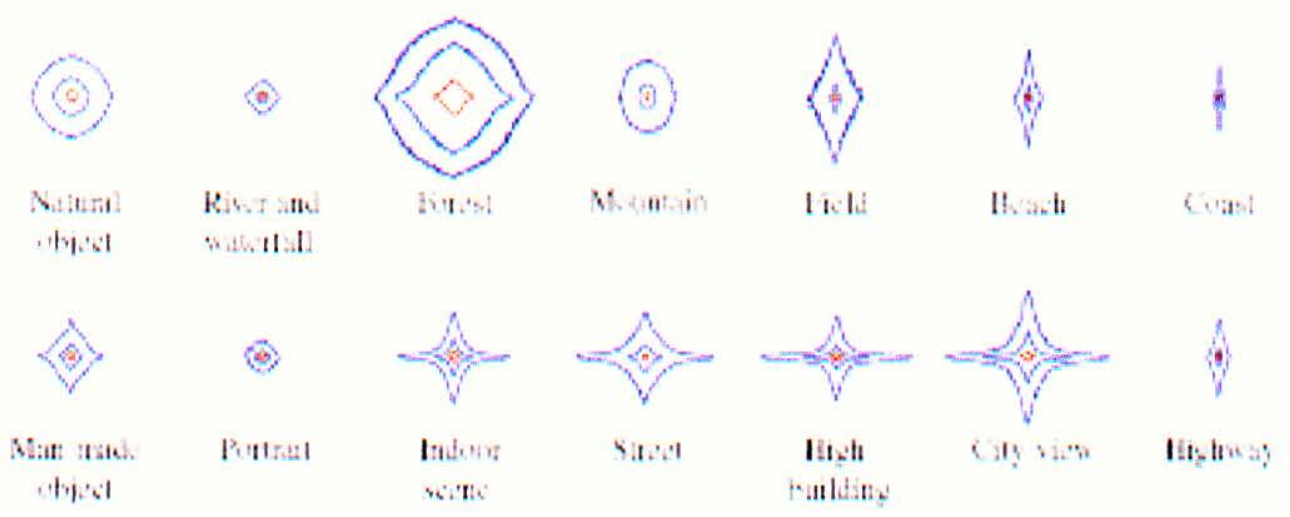

Figure 4. Figure taken from Torralba and Oliva (2001) depicting the different 'spectral signatures' of images taken from different types of real-world environments. Each example is a contour plot of the average spectrum from each image set. The three contour lines, from inside to outside, of each plot represent 60,80 , and $90 \%$ of the energy of the spectral signatures. Again, note that biases in the horizontal direction depict biases of vertical content in the spatial domain. 

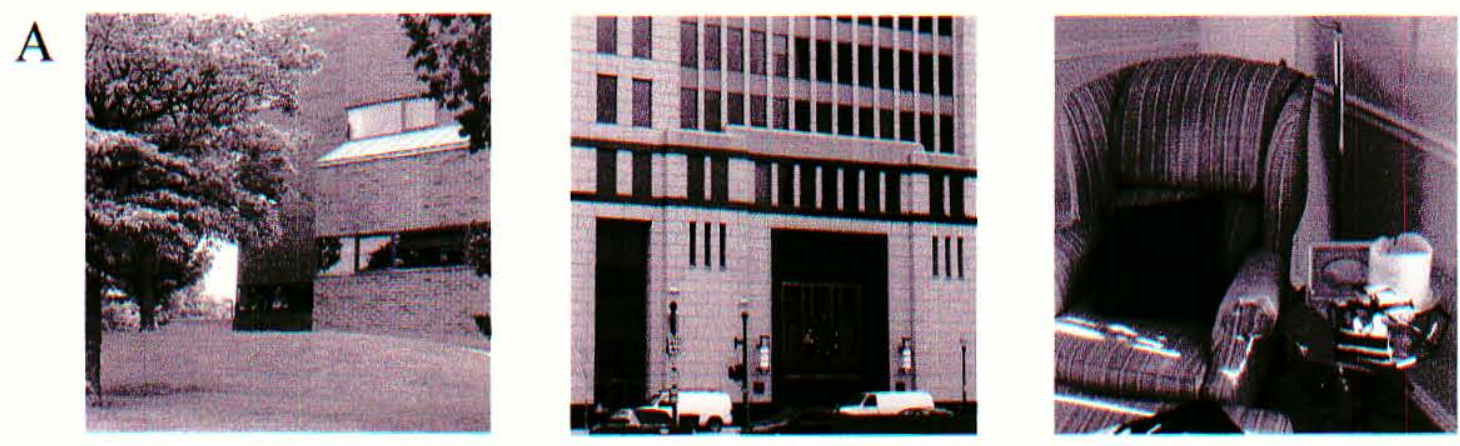

B
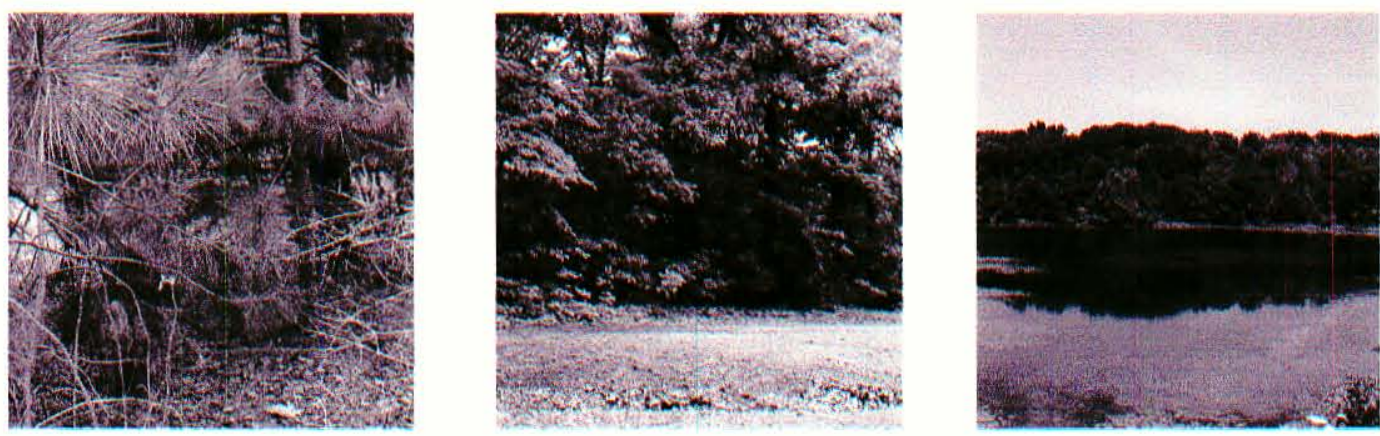

Figure 5. A) Far left: typical image in the naturalistic-carpentered content image set; Middle: example of a typical outdoor image from the carpentered image content set; Right: typical indoor image from the carpentered image content set. B) From left to right, typical close-range, mid-range, and far-range naturalistic-content imagery (Hansen \& Essock, 2004b). 


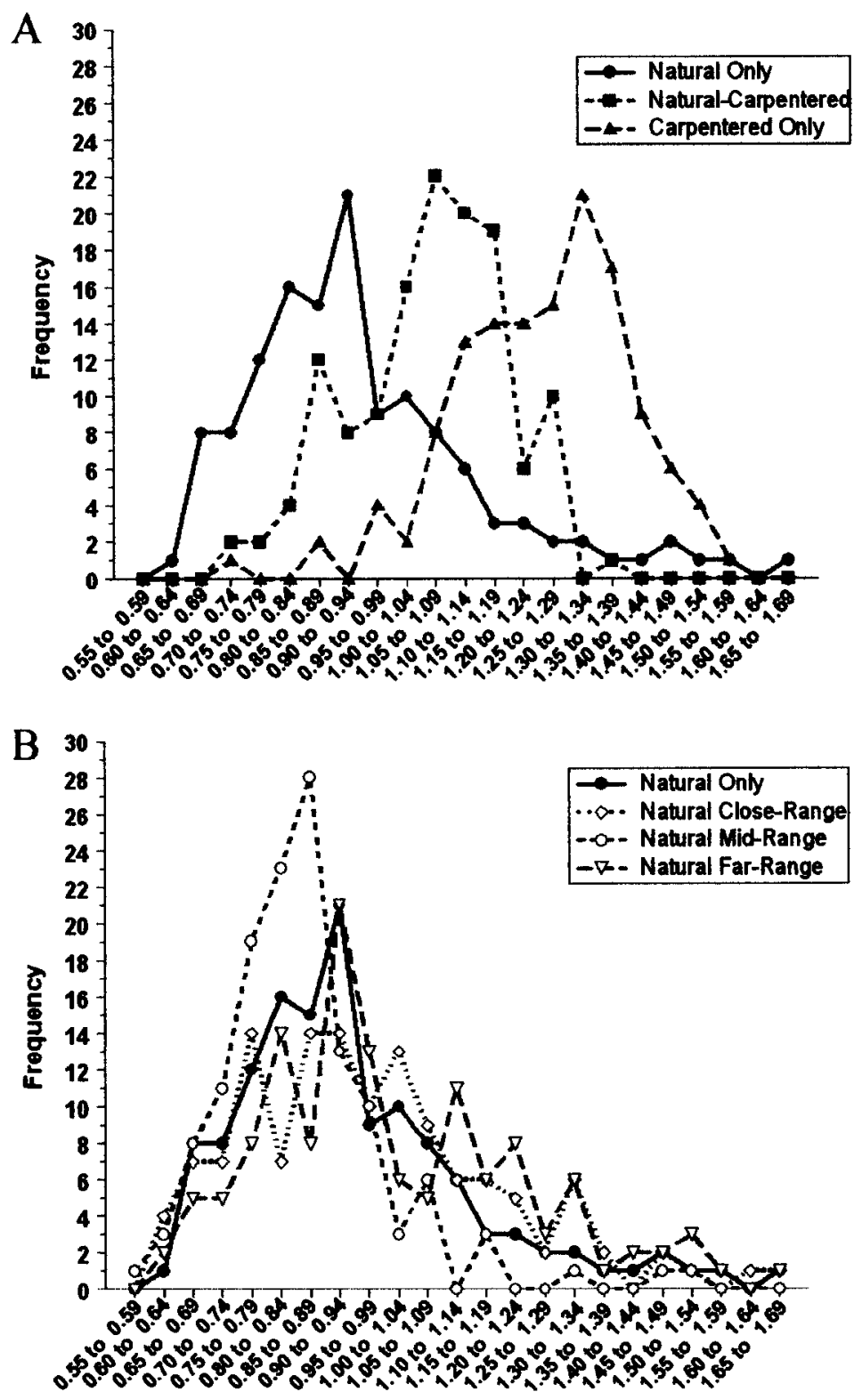

Figure 6. A) Frequency curves showing the distribution for each of the three different image sets' $\alpha$ values (naturalistic, naturalistic-carpentered mix, and carpentered image sets). On the abscissa is plotted the full range of $\alpha$ values observed in the sample, where each tick mark represents a small range ( .05$)$ in which the frequency of occurrence of imagery possessing corresponding $\alpha$ values that fell into a given range was measured. On the ordinate is the number of images (i.e., frequency) in each of the $\alpha$ bins represented on 
the abscissa. B) Frequency curves showing the distribution for each of the three different image range sets' $\alpha$ values (close-range, mid-range, and far-range sets). The solid curve is re-plotted from (A) to serve as a reference to the original naturalistic-content sample (Hansen \& Essock, 2004b). 


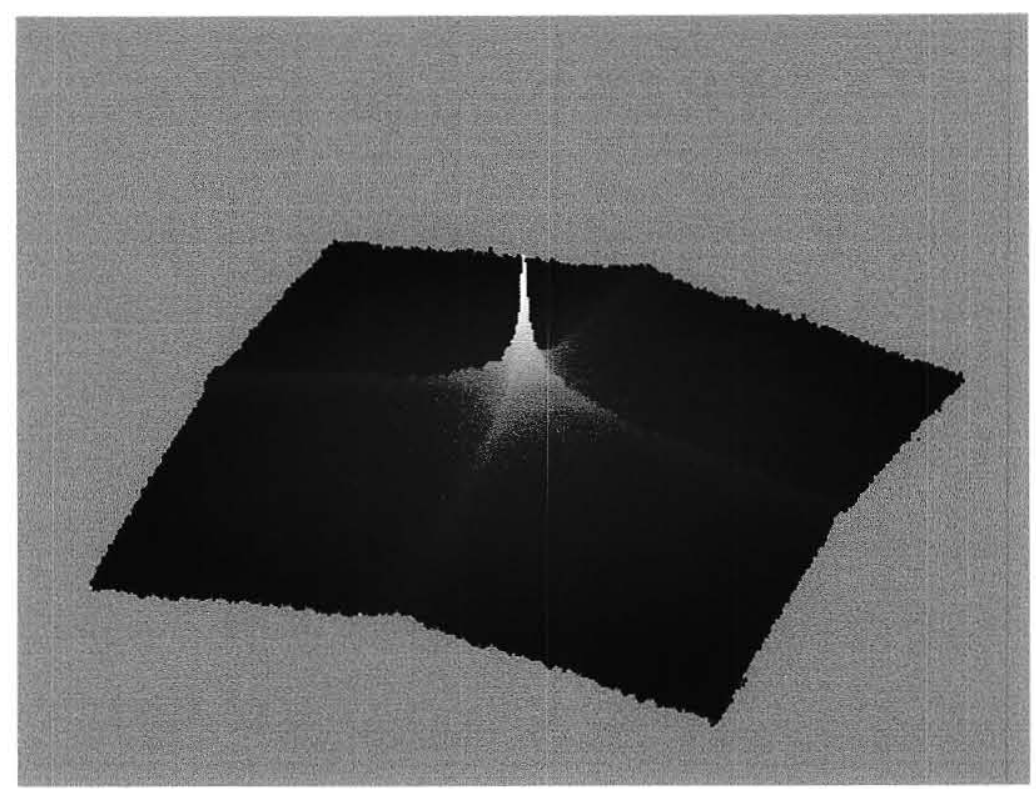

Figure 7. Two-dimensional representation of an amplitude spectrum (spatial frequency increases with radius, orientation changes with theta) obtained by averaging 70 exemplar spectra; note the concentration of amplitude is along the horizontal and vertical axes (Hansen \& Essock, 2004b). 

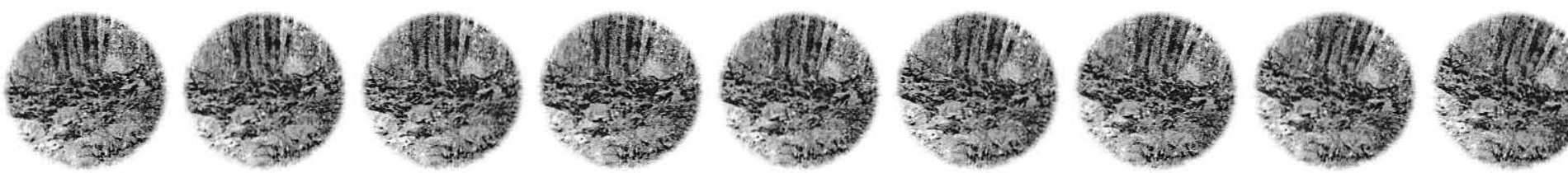

$\frac{1}{3}$

Whas
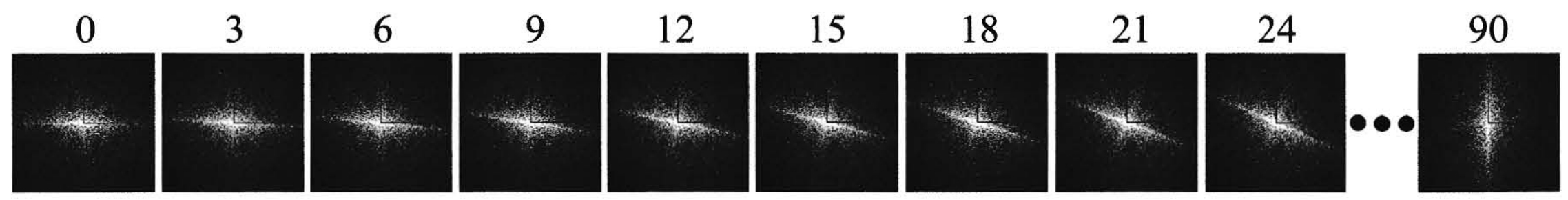

Figure 1. Schematic depicting the process involved in calculating the amplitude biases at 61 orientations (i.e., $0^{\circ}$ through $180^{\circ}$ ) (Hansen \& Essock, 2004a). The top row shows a series of rotations for an exemplar scene starting at $0^{\circ}$ (camera aligned) to $90^{\circ}$. The bottom row shows each amplitude spectrum corresponding to its respective spatial image. The two red lines drawn on each spectrum indicate the $0^{\circ}$ and $90^{\circ}$ vectors that were taken from each image rotation. Note that for the un-rotated image sample and the full $90^{\circ}$ rotated image sample, camera-aligned vertical $\left(0^{\circ}\right)$ and horizontal $\left(90^{\circ}\right)$ content will be sampled twice. Here, vertical $(0$ on the abscissa) was taken from the un-rotated sample and horizontal (90 on the abscissa) was taken from the full $90^{\circ}$ rotated image. Since $0^{\circ} / 180^{\circ}$ correspond to the same spatial content, the same vector sample (from the un-rotated image) was used for both (refer to the text for further details). 


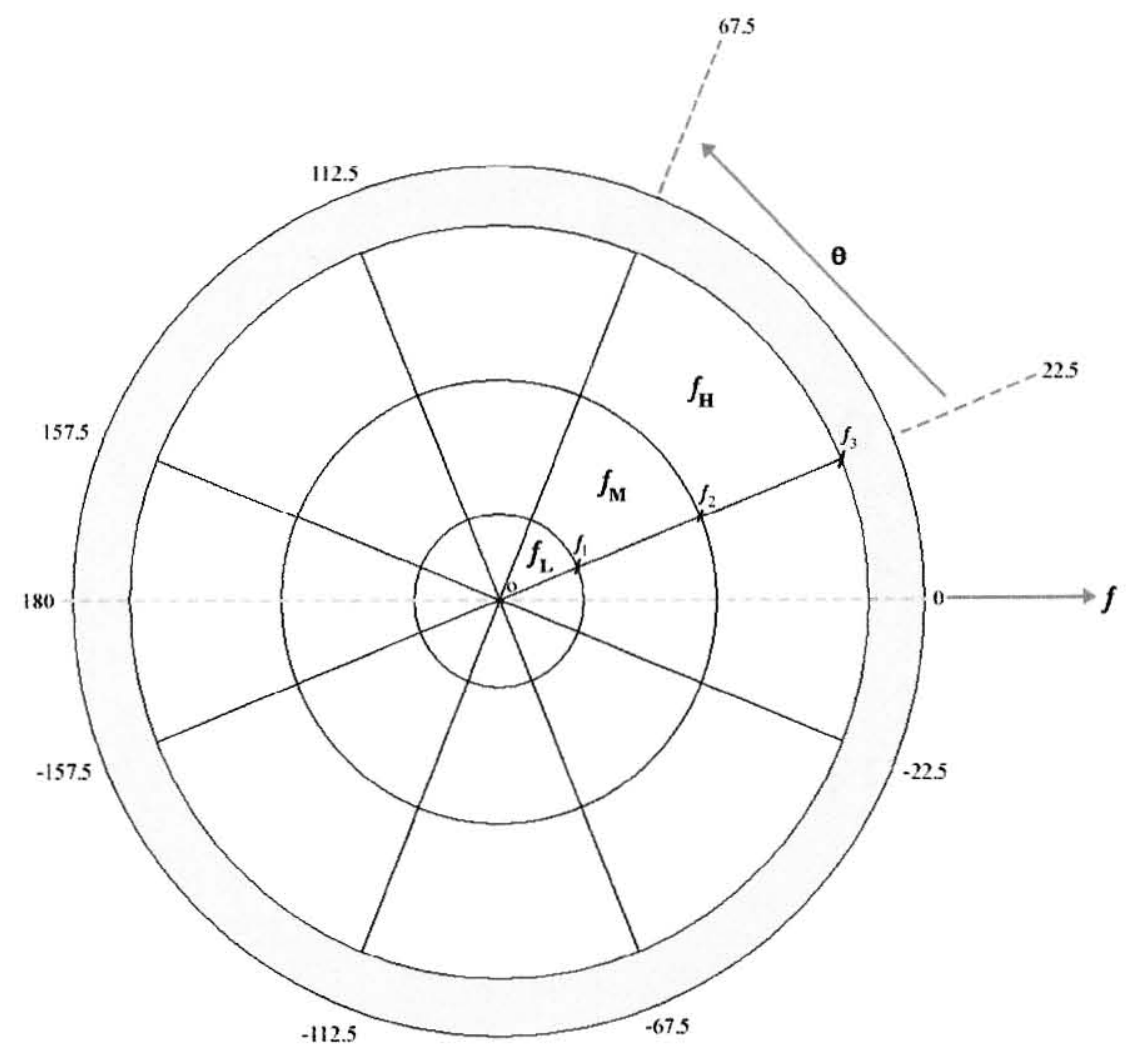

Figure 9. Schematic depicting the parsing of a given amplitude spectrum in order to calculate and classify image content biases (Hansen et al., 2003). Each spectrum was first parsed into four $45^{\circ}$ wide orientation bands $\left(\theta_{\text {vert, }}, \theta_{\mathrm{d} 45}, \theta_{\text {horz }}, \theta_{\mathrm{d} 135}\right)$, then further subdivided into three 2-octave frequency bands $\left(f_{\mathrm{L}}, f_{\mathrm{M}}, f_{\mathrm{H}}\right)$. The shaded region represents the $\mathrm{A}(f, \theta)$ coordinates beyond the Nyquist limit not included in oriented amplitude bias calculation. This figure has been drawn to depict how orientation biases of amplitude were calculated and thus is not drawn to scale. 


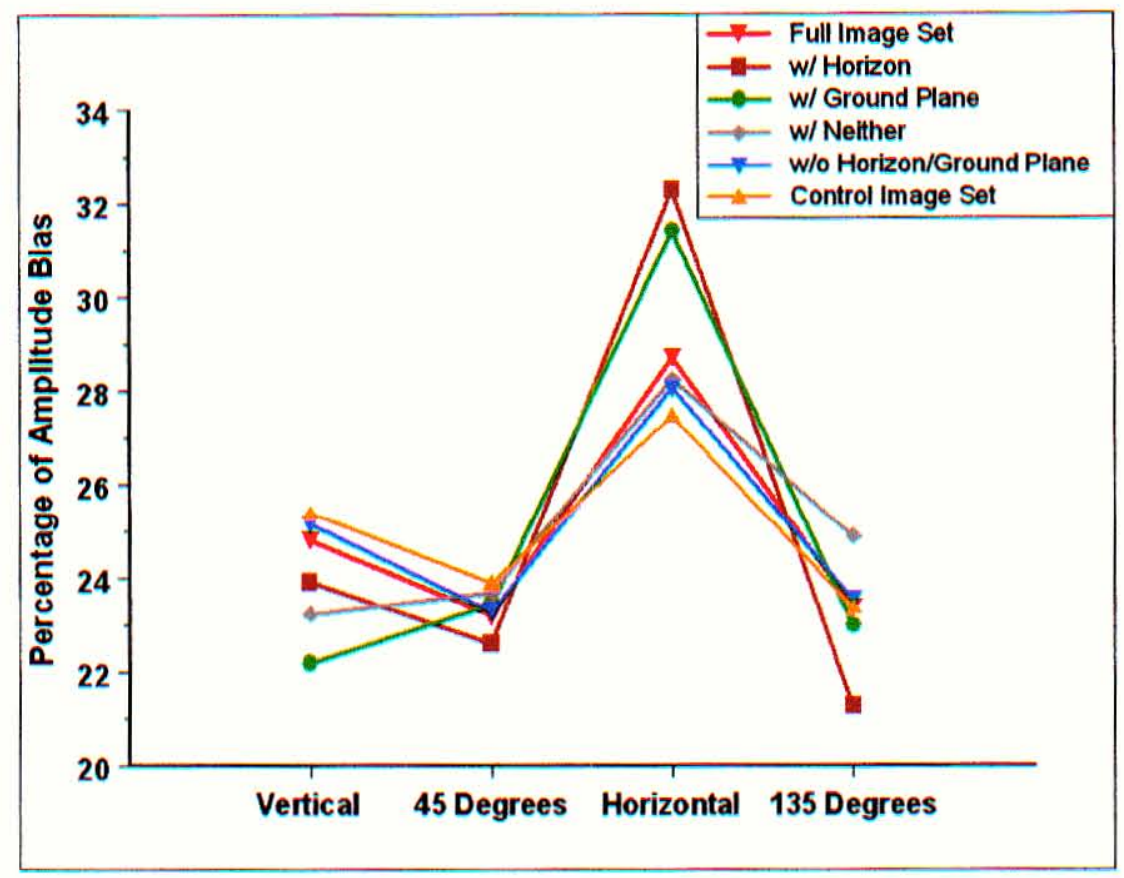

Figure 10. Orientation analysis of natural scene content. Plotted is the average ratio of amplitude at a given orientation relative to the other orientations. The three categories: "w/Horizon", "w/Ground-plane", or "w/Neither" plot the averages from subsets of images containing a clear horizon; containing ground-planes consisting of various textures; or containing general foliage and shrubbery, thus containing neither a horizon or a ground plan. Also plotted are the measurements made over the entire 231 natural scene image set (Hansen \& Essock, 2004a) from which the subsets were drawn (“All Images"), and the set of all images remaining after those containing a horizon or a ground plane were removed ("w/o Horizon/Ground Plane"). Finally, measurements made on a control set of images obtained by a different lab (see text). Note that all six conditions show a strong horizontal bias. Second most prevalent is vertical content in typical scenes (although the vertical bias is not present in scenes of uniform ground planes (where horizontal dominates) or of general shrubbery). 


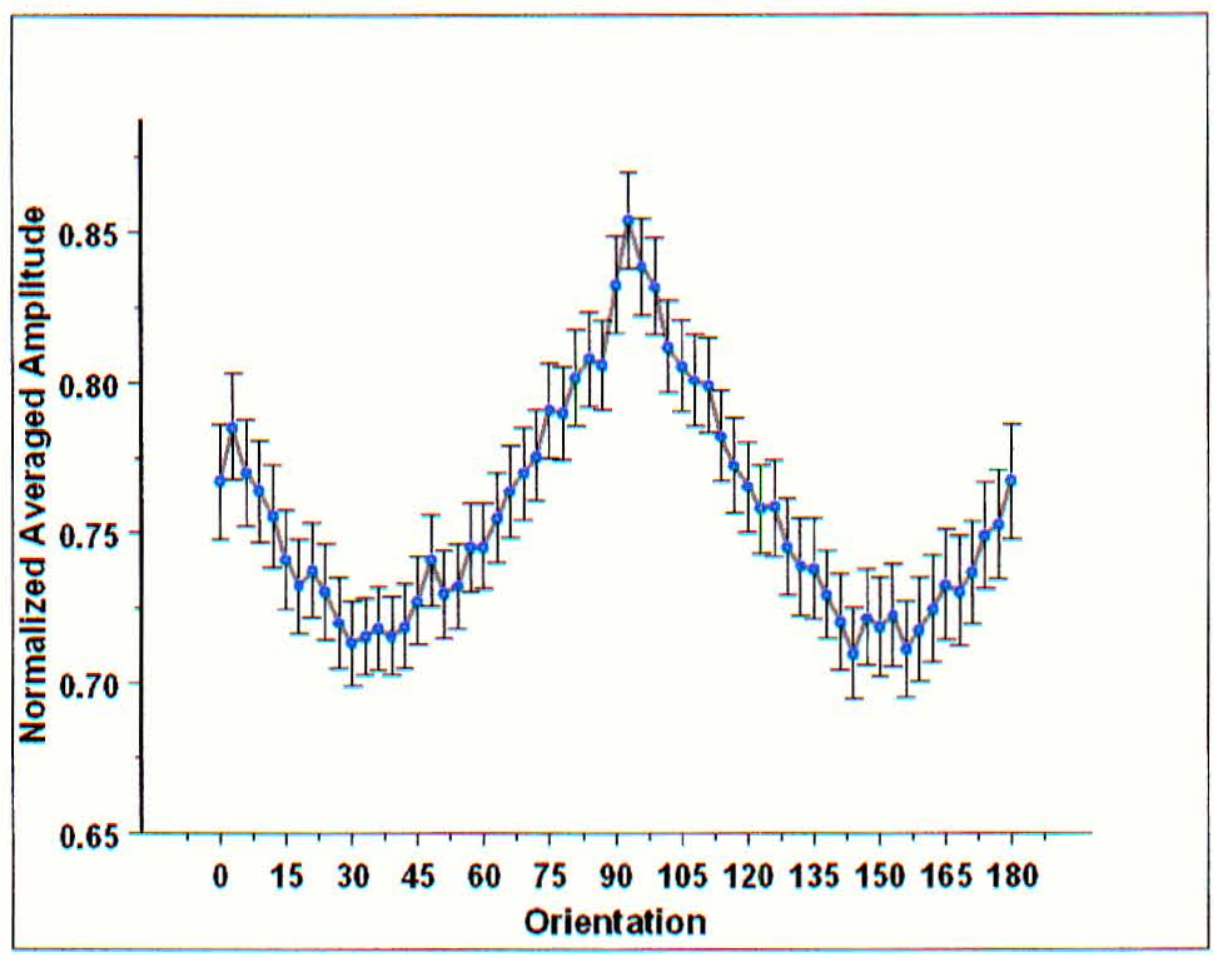

Figure 11. Average normalized amplitude for each of the sampled orientations described in the text. Each point on this plot represents the average over the 60 images of the vector for that orientation (abscissa) summed across spatial frequency. Note that the orientations with the most amplitude are located at or near horizontal (here $90^{\circ}$ corresponds to horizontal spatial content) (Hansen \& Essock, 2004a). 

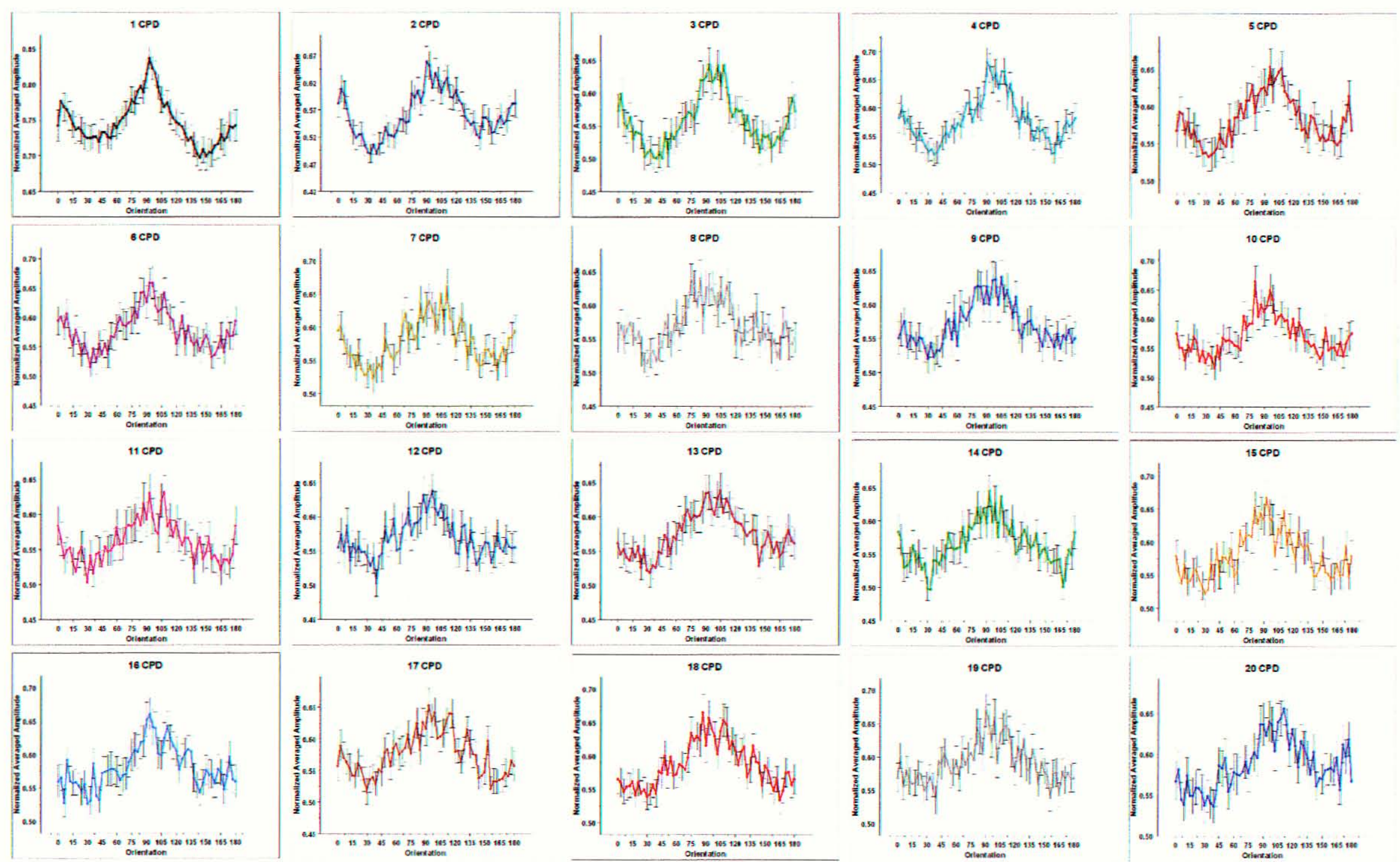
Figure 12. Average normalized amplitude bias for each of the sampled orientations, mentioned in the text, plotted with respect to spatial frequency (cycles per degree). Each point on these plots represents the average summed vector segment for that orientation (abscissa) and respective spatial frequency averaged across the 60 images (refer to text for further details). Note that the bias in amplitude at or near horizontal orientations is clearly present at all spatial frequencies, and that the second peak at or near vertical orientations is largest at the lower spatial frequencies and diminishes towards higher spatial frequencies (Hansen \& Essock, 2004a). 

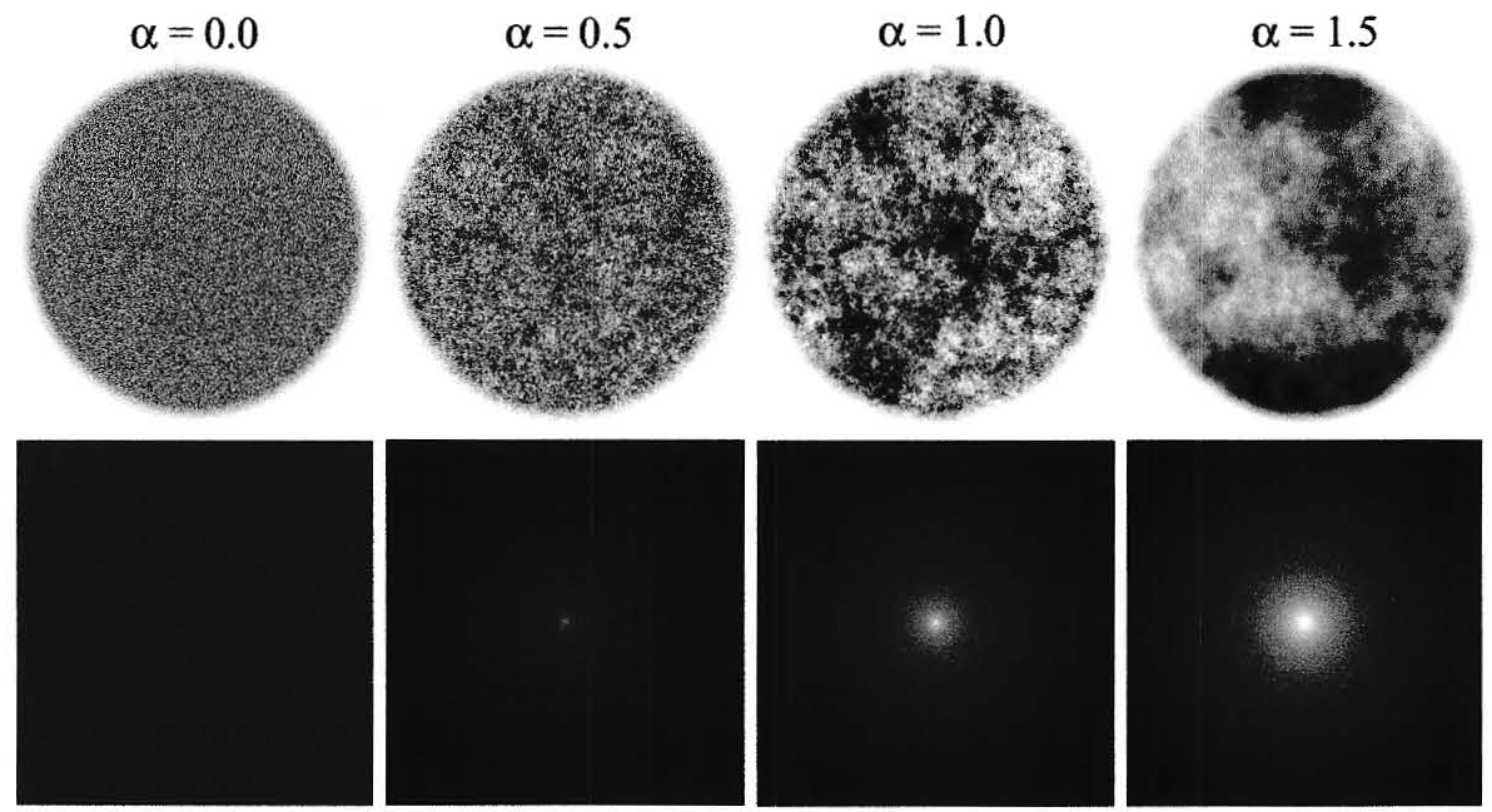

Figure 13. Top row: Examples of broadband visual noise pattern that have random phase spectra and different amplitude spectrum slopes. Bottom row: Examples of the amplitude spectra used to form the spatial noise patterns shown in the top row. 


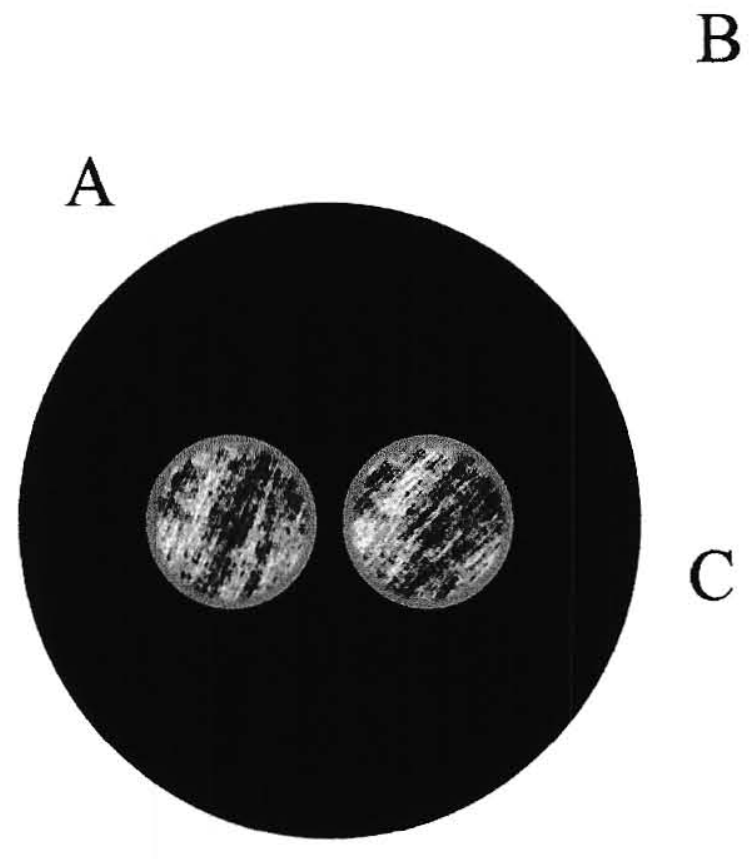

D
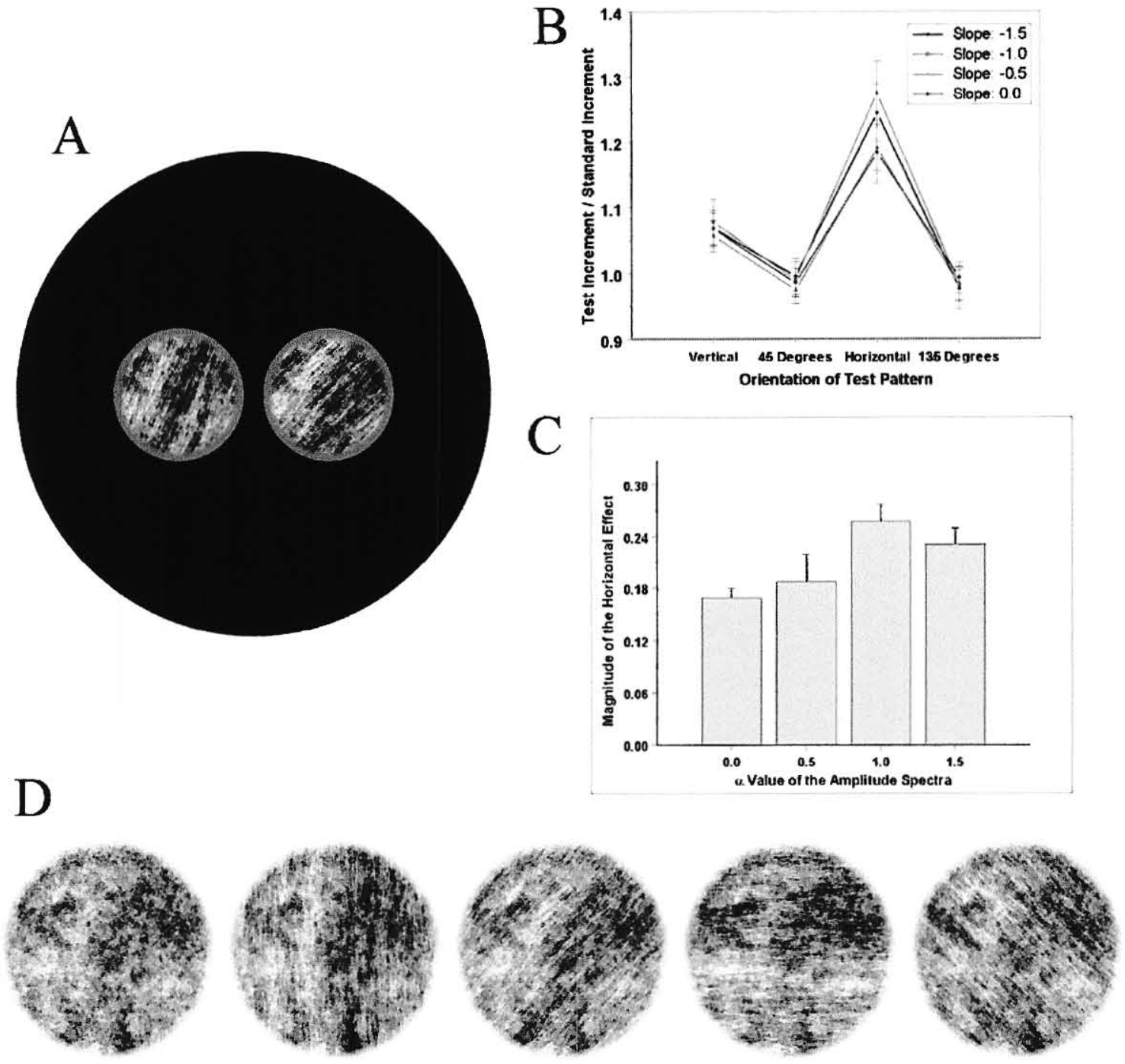

Figure 14. A) Illustration of the mask used in the matching experiments of Essock at el. (2003). B) Data re-plotted from Essock et al. (2003), note that higher values indicate poor perceptual salience (less sensitivity). C) Histogram created using the matching data from Essock et al. (2003) showing the change in the magnitude of the horizontal effect for the different amplitude spectrum slopes. D) Examples of a visual noise pattern containing an oriented increment in amplitude at one of the four test orientations mentioned in the text. From left to right, isotropic visual noise pattern with no oriented 
increment; vertical increment of amplitude; $45^{\circ}$ increment of amplitude; horizontal increment of amplitude; and $135^{\circ}$ increment of amplitude. 

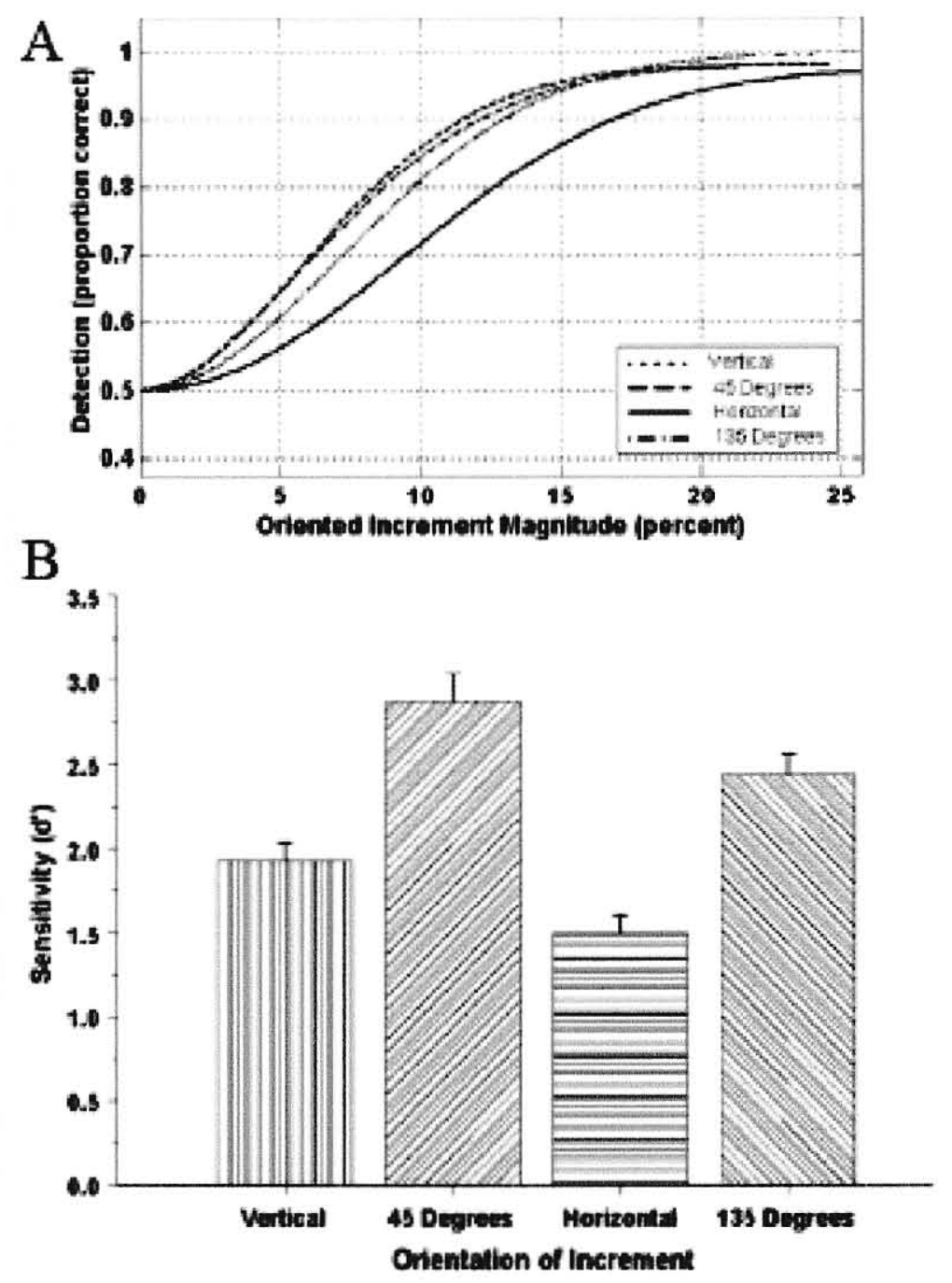

Figure 15. A) Data re-plotted from Essock et al. (2003) showing the horizontal effect for the 2-AFC threshold paradigm described in the text. B) Data re-plotted from Essock et al. (2003) showing the horizontal effect for the single interval Yes/No near-threshold paradigm described in the text. 

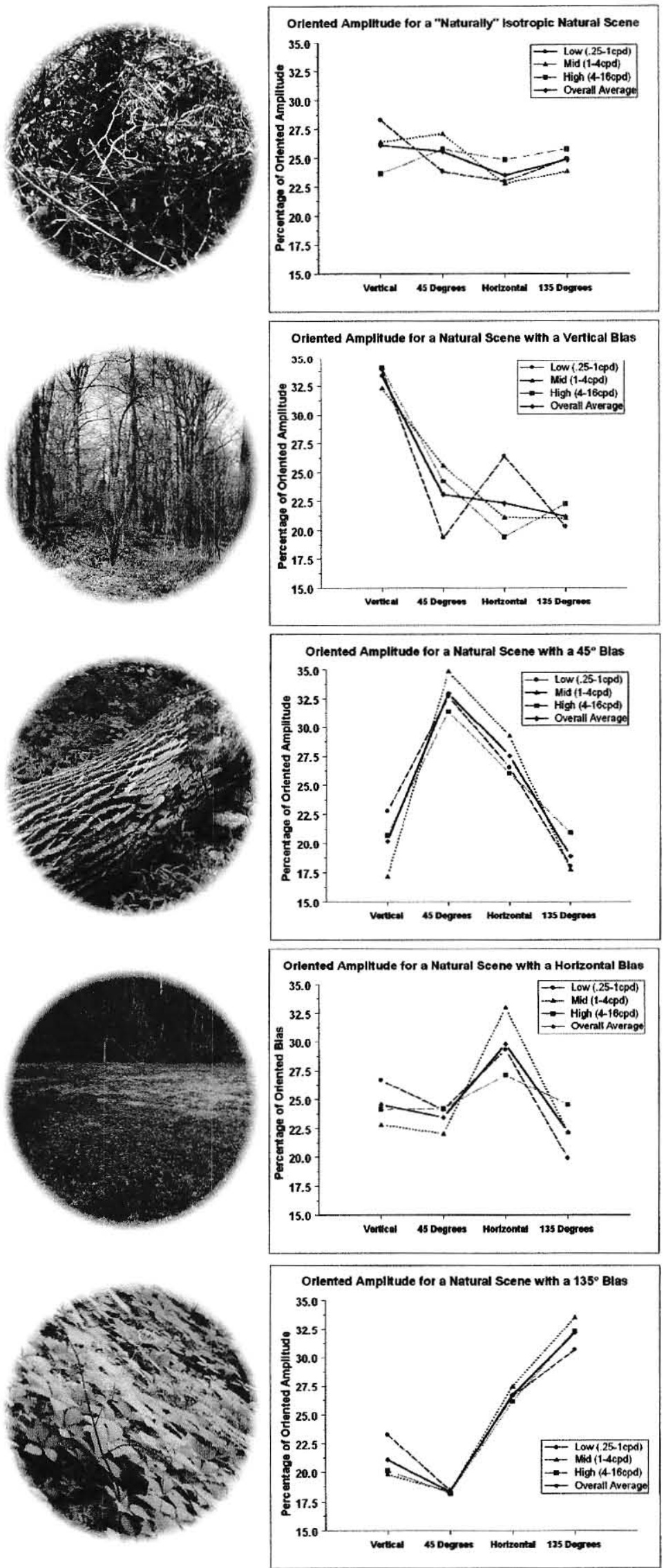
Figure 16. To the left of each graph is an example image from the different scene content bias subsets, arranged left to right in the following order: no predominant bias in content at any of the four test orientations; a scene with a bias in $45^{\circ}$ amplitude; a scene with a bias in vertical amplitude; a scene with a bias in horizontal amplitude; and a scene with a bias in $135^{\circ}$ amplitude. The graphs themselves are plots of the oriented amplitude bias for each image. The ordinate plots the relative percentage of amplitude bias (Hansen et al., 2003). 

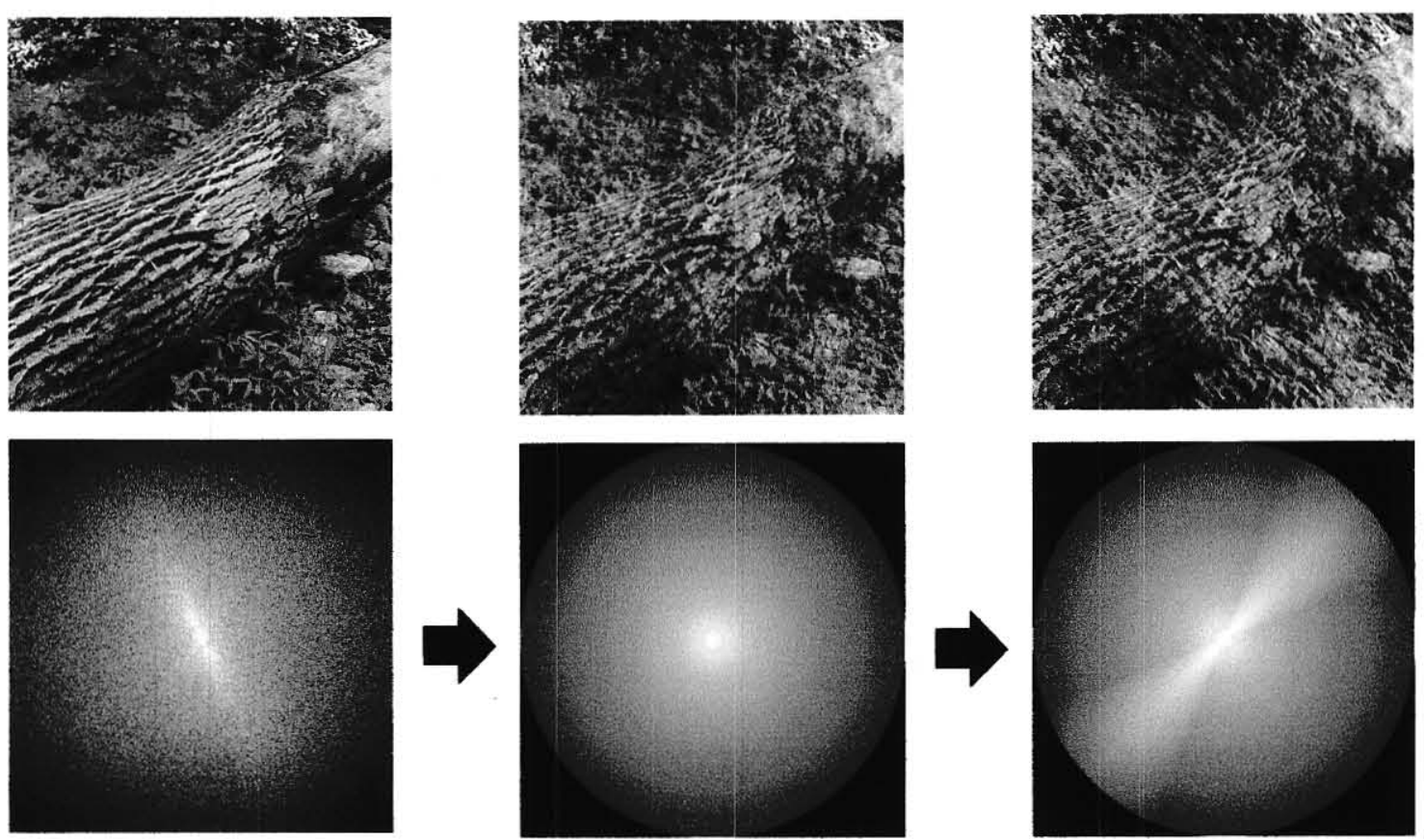

Figure 17. An example of the method by which an oriented increment in amplitude was applied to each image as mentioned in the text. The example runs from left to right, with the top row showing the resulting spatial image containing the manipulation in the frequency domain. Starting left-most with an unaltered image (Top left) and corresponding amplitude spectrum (Bottom left); Bottom middle: after the spectrum was made isotropic; Bottom right: after the amplitude spectrum has been made to contain an oriented increment in amplitude at $135^{\circ}$ with the triangle filter described in the text. 

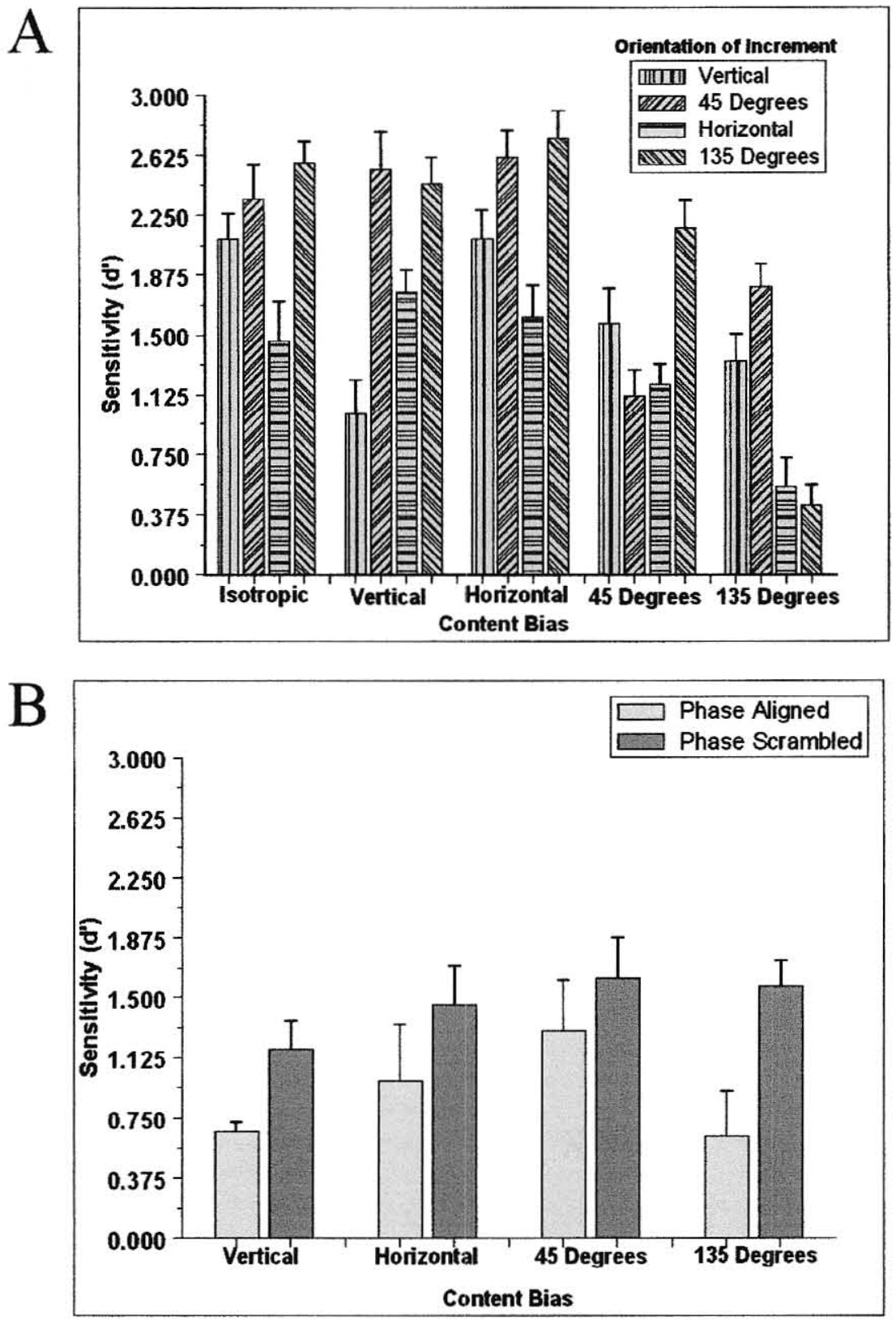

Figure 18. Results re-plotted from Hansen et al. (2003). A) The abscissa plots averaged observer data (and averaged within subject SEM), showing performance for detecting an oriented increment in amplitude at each of the four test orientations for each image content type subset. For example, the grouping labeled 45 degrees on the abscissa plots averaged observer sensitivity (d') for detecting an oriented increment of amplitude at 
each of the test orientations with scenes that were dominated by image content at $45^{\circ}$. B) The abscissa is grouped by scene subset type, and indicates observer sensitivity (d') for detecting an oriented increment of amplitude at the same orientation as the scenes' content bias for images where the phase relations carrying the oriented bias were "aligned", and when they had been randomized as described in the text. 


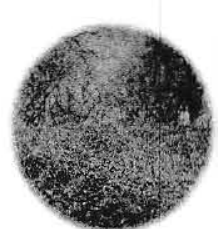

0.659

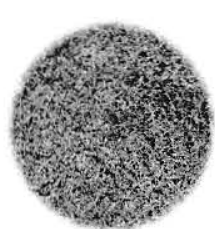

$\mathbf{0 . 7 2 5}$

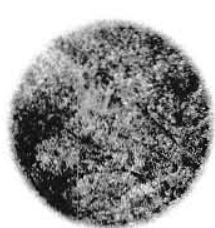

$\mathbf{0 . 8 5 4}$

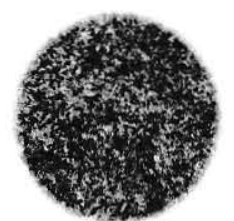

0.949

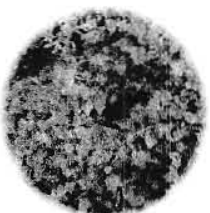

1.061

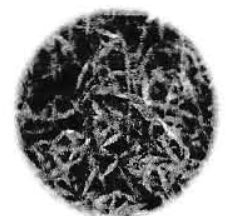

1.443

Figure 19. Stimulus examples from each $\alpha$ image set used in Hansen and Essock (2004b); the numbers at the bottom indicate the respective $\alpha$ value from which the cxamples were selected. The images have had their amplitude spectra made isotropic (by the method described in Figure 3, but do not contain an oriented increment of amplitude. 

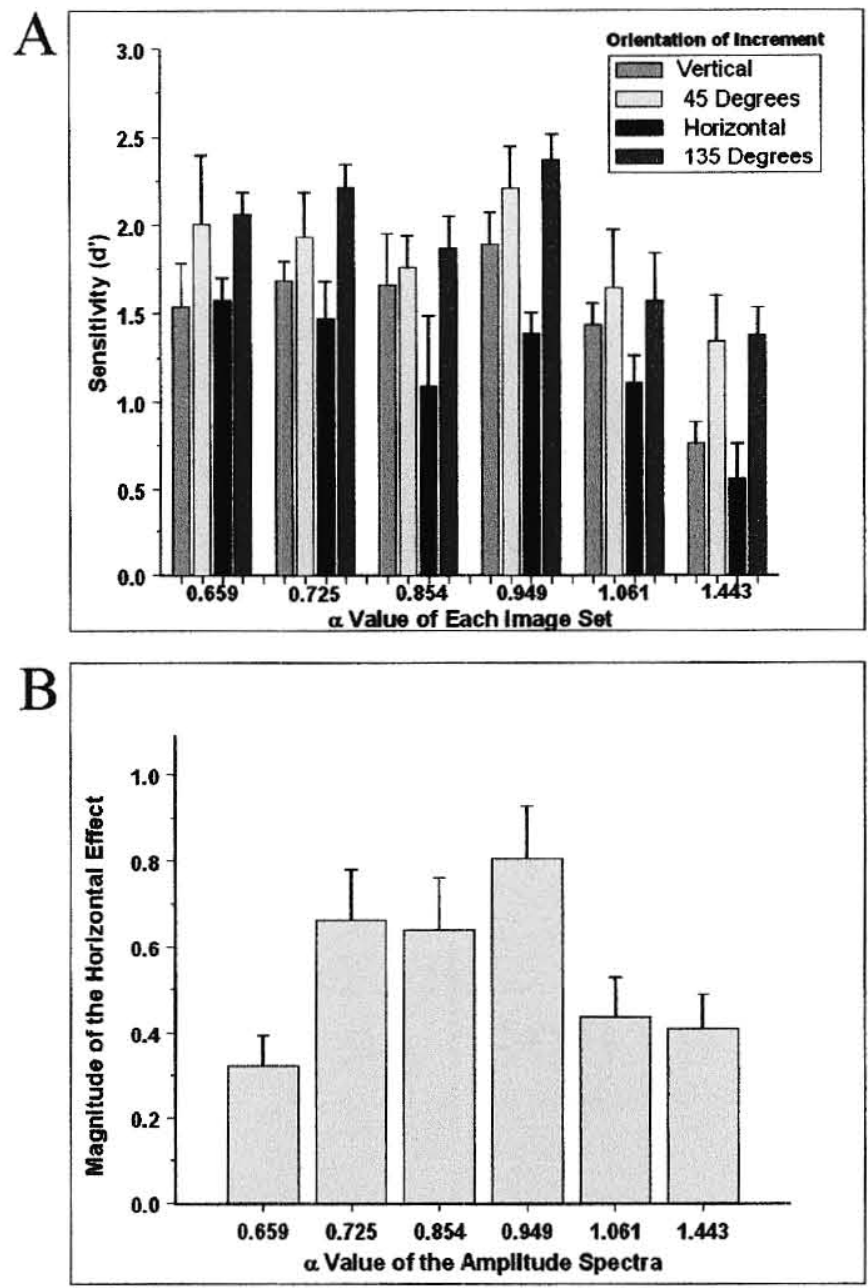

Figure 20. Data re-plotted from the variable slope experiments conducted by Hansen and Essock (2004b). A) On the ordinate is average sensitivity (d') for detecting oriented increments of amplitude (indicated by bar shading) with imagery contained in each $\alpha$ image set (as labeled on the abscissa). Error bars are +1 S.E.M. B) Histogram showing the magnitude of the horizontal effect (i.e., the difference between the average ratio for vertical, $45^{\circ}$ - and $135^{\circ}$-oblique matches and horizontal matches) for each of the $\alpha$ conditions (obtained by first averaging across experimental and control conditions); error bars are +1 S.E.M. 

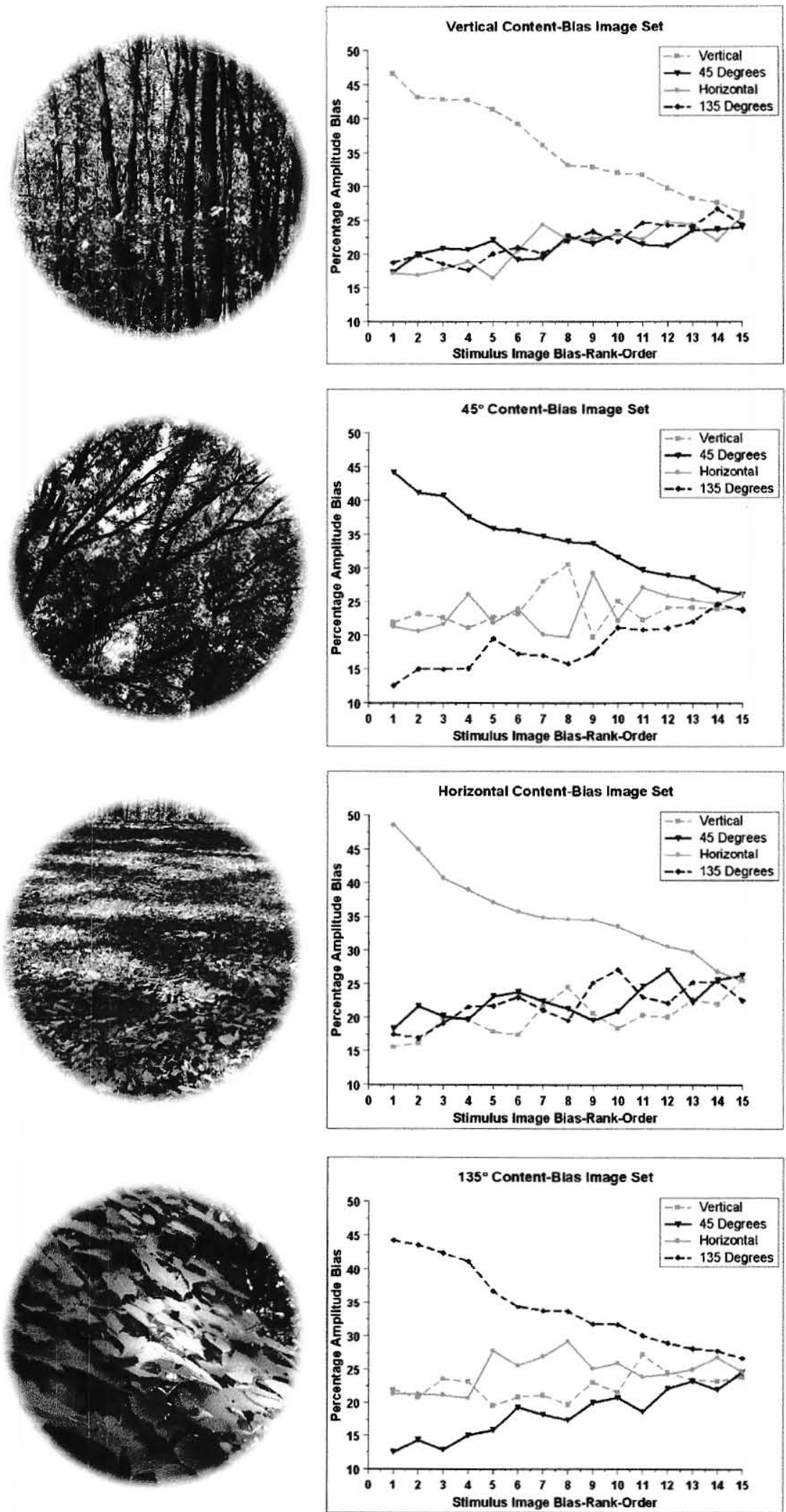
Figure 21. An example of an image (un-altered) from each of the variable orientation content-bias image sets (left column) and graphs depicting the decreasing amplitude at each orientation across the set of images (right column) (Hansen and Essock, 2004b). All graphs are plotted in an identical fashion, with percentage of amplitude bias (i.e., ratio of the amplitude within a $45^{\circ}$ 'wedge' region centered at one of the four orientations to the amplitude at the other three orientations) on the ordinate. For the abscissa, images were rank ordered with respect to amplitude bias for each of the four content biased sets in order to illustrate the decline in a given orientation's content bias across the images contained in each set. 


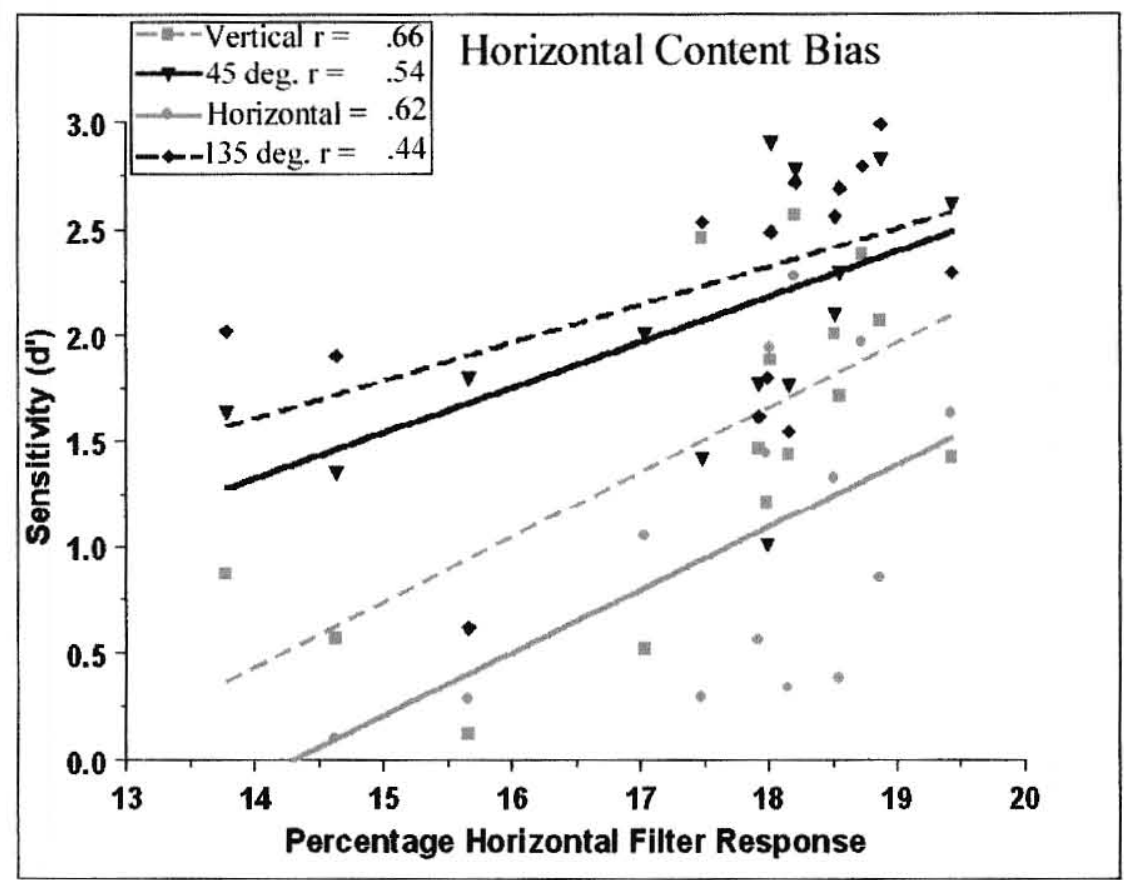

Figure 22. Data re-plotted from the variable horizontal content-bias stimulus set used by Hansen and Essock (2004b). The graph plots performance for detecting each of the four oriented increments of amplitude (on the ordinate) against D6 filter response ratios obtained from filters that were aligned with the orientation of the image's content bias (on the abscissa) as described in the text. That is, performance for detecting different oriented increments (for each image in a set) is plotted against the amount of oriented content-bias (for that image) for the variable horizontal content-bias stimulus set. 

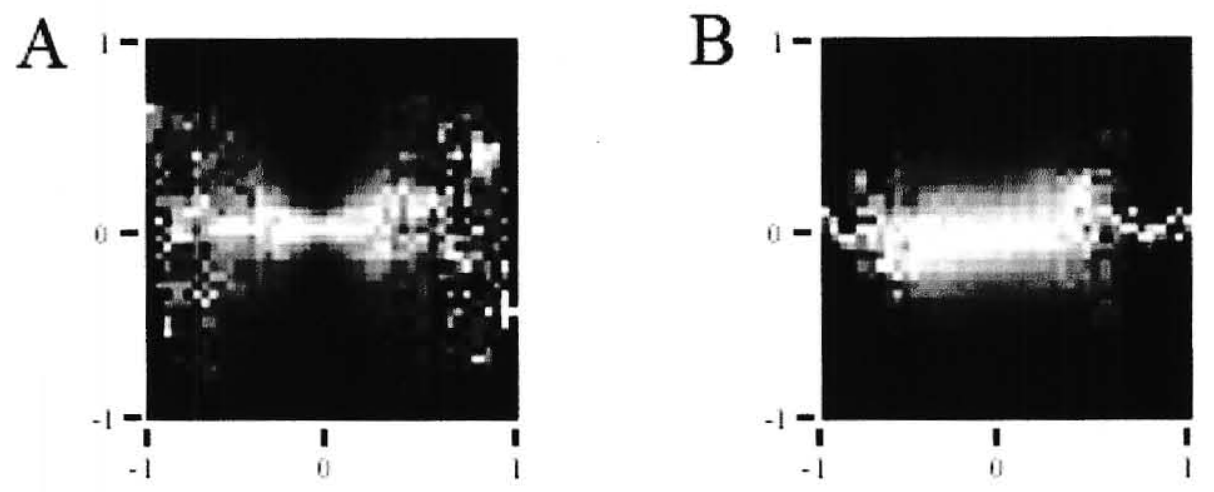

Figure 23. Example 2D joint histograms obtained. A) Example of an ideal 2D joint histogram showing a high dependency between the given 'primary' filter response (abscissa) and a 'secondary' filter response (ordinate). B) Example of a typical 2D joint histogram plotted between 'primary' and 'secondary' filter responses; note the lack of the characteristic "bow-tie" pattern, indicating a lack of filter response dependency. 

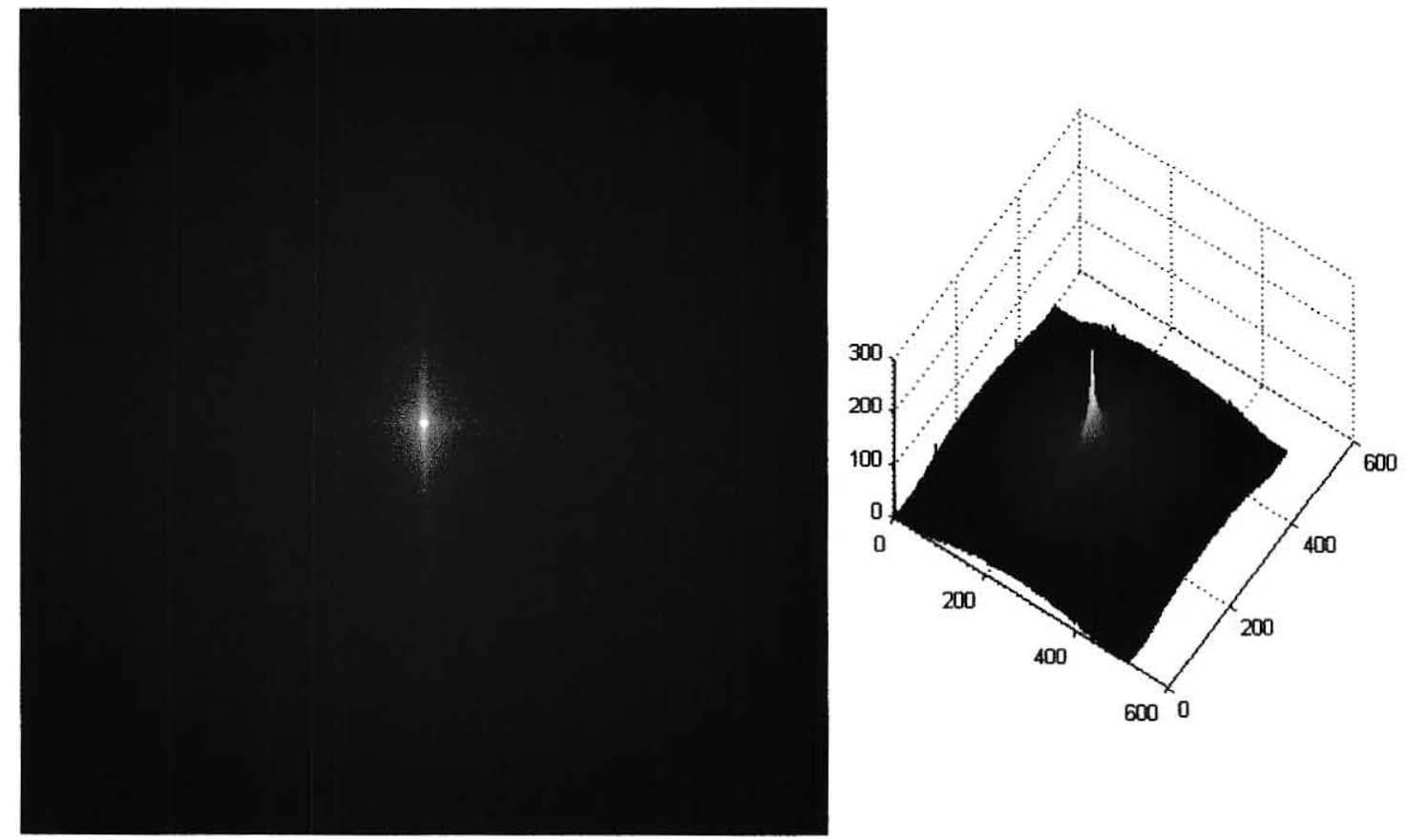

Figure 24. On the left is an average spectrum obtained by averaging 88 amplitude spectra from images containing a bias in the amount of horizontal content, note the sharp peak along the vertical axis (i.e., representing horizontal in the spatial domain). On the right is the same figure plotted in 3D space in order to better show the triangular peak of the amplitude bias. 


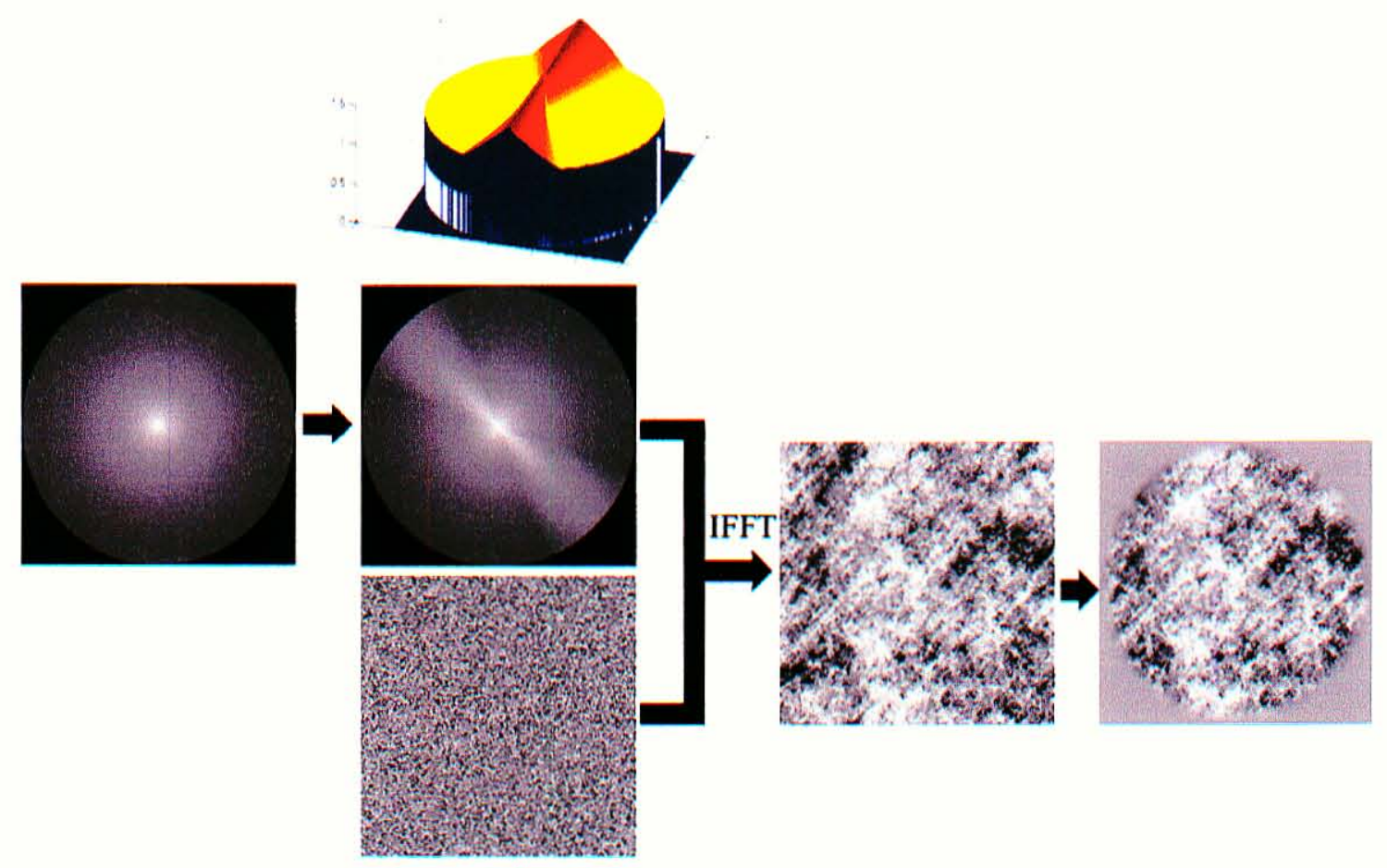

Figure 25. An illustration of the procedures that were carried out in generating the test stimuli for the set of conditions in Experiment 1. From left to right, the isotropic amplitude spectrum $(\alpha=1.0)$, the amplitude spectrum weighted by the triangle filter described in the text (3D plot of that filter is shown at the top) which is then combined with the random phase spectrum (shown at the bottom) during the Inverse Fast Fourier transform (indicated by the IFFT). An example of the noise pattern with a broadband increment at the $45^{\circ}$ orientation in the spatial domain, followed by an illustration of the same pattern fit with the edge-'blurred' circular window described in the text. 


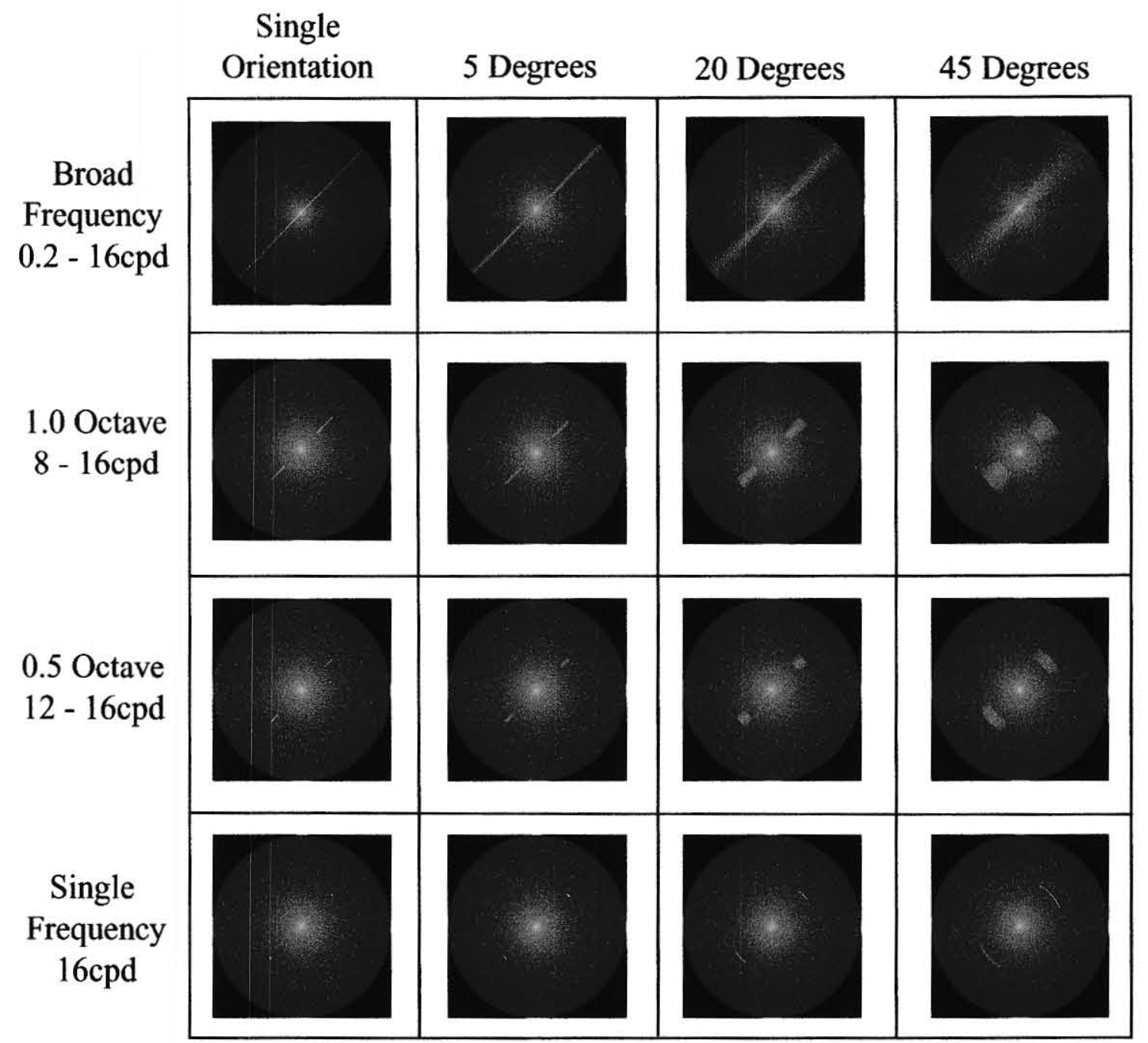

Figure 26. A 16-cell matrix where each cell contains an example of a triangle filter at an exemplar orientation limited in the spatial frequency, orientation or both spatial frequency and orientation directions. Each cell represents the different triangle filter extents that will be used in each of the proposed conditions of Experiment 1. Note that these same extents will be used in Experiment 2, only the area outside the depicted filters will be set to zero, thus those experiments will be evaluating the perceptual salience of different amounts of oriented content in the absence of a broadband background (refer to the text for further details). 

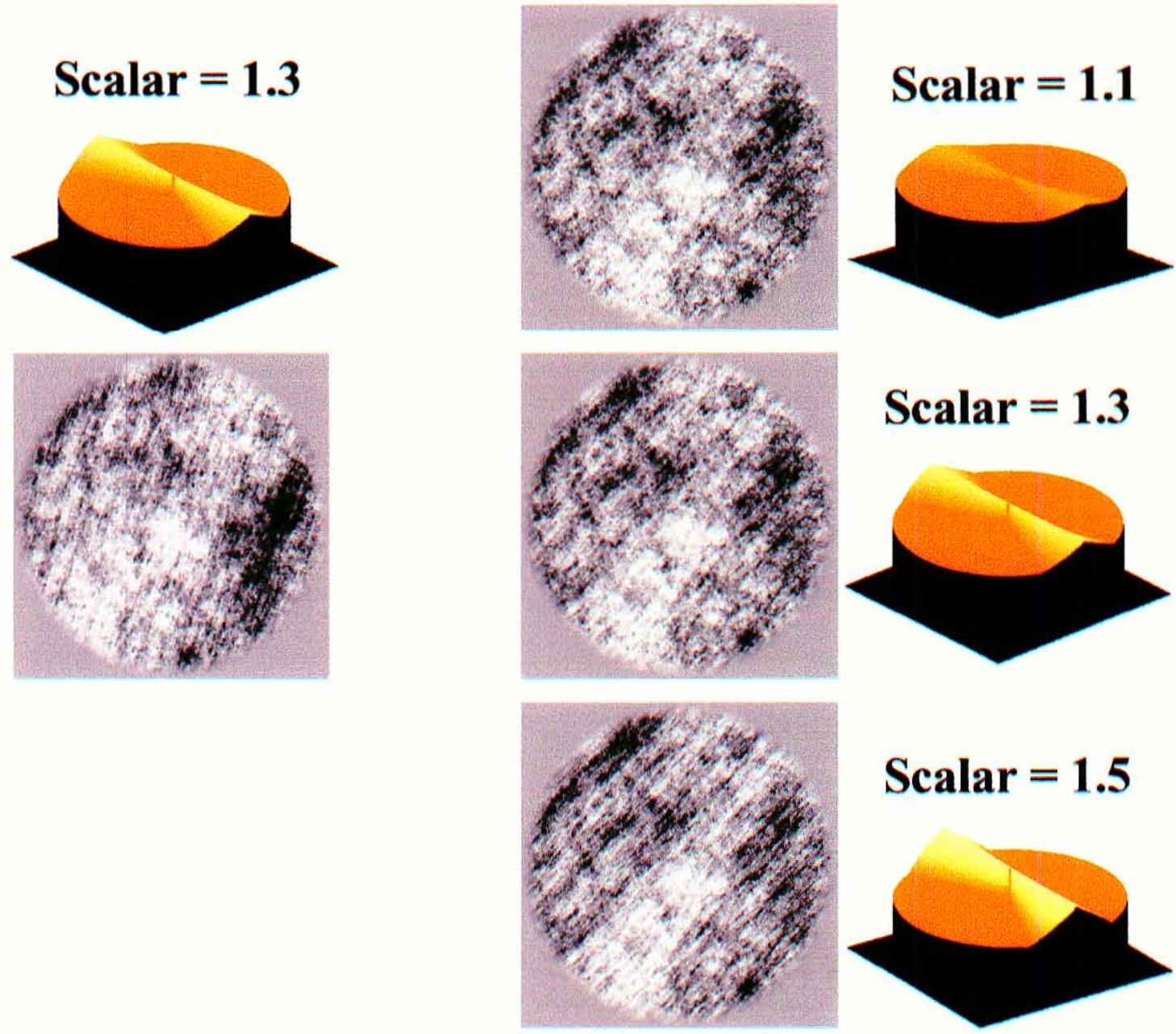

Figure 27. Diagram representing what the participants were actually adjusting when making their suprathreshold matches. On the left is a 3D representation of an amplitude spectrum for the spatial noise pattern shown underneath (note that the spectrum has been made to be flat in order to better show the magnitude of the triangle increment). The raised triangular portion of that spectrum is a result of the triangle increment filter that was assigned a scalar value of 1.3. The noise pattern on the left is an example of one of the standard patterns containing oriented structure (resulting from the triangle filter increment) to which participants were asked to make a perceptual match by adjusting the amount of the oriented structure in the test pattern. On the right are three examples of a 
given test noise pattern with triangle increments varying in magnitude (i.e., scalar magnitude). Thus, while the participants were matching the amount of oriented bias in the noise patterns in the spatial domain, in the Fourier domain they were actually matching the magnitude of the triangle increment. 


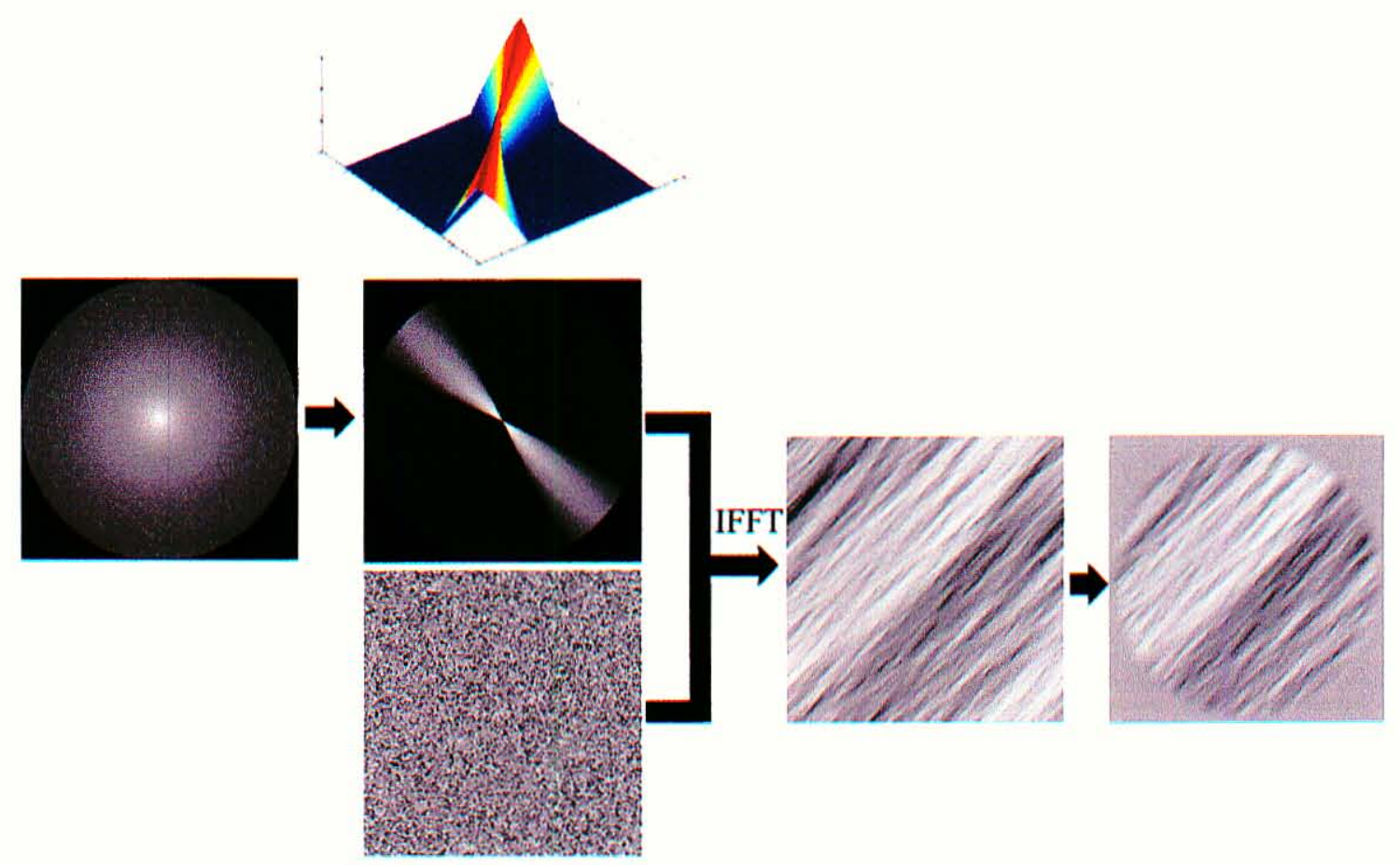

Figure 28. An illustration of the procedures that were carried out in generating the test stimuli for the set of conditions in Experiment 2. From left to right, the isotropic amplitude spectrum $(\alpha=1.0)$, the amplitude spectrum weighted by the triangle filter described in the text and the area outside of this region is set to zero (3D plot of that filtering process is shown at the top) which is then combined with the random phase spectrum (shown at the bottom) during the Inverse Fast Fourier transform (indicated by the IFFT). An example of the spatial pattern with a broadband increment at the $45^{\circ}$ orientation in the absence of a broadband background, followed by an illustration of the same pattern fit with the edge-'blurred' circular window described in the text. 

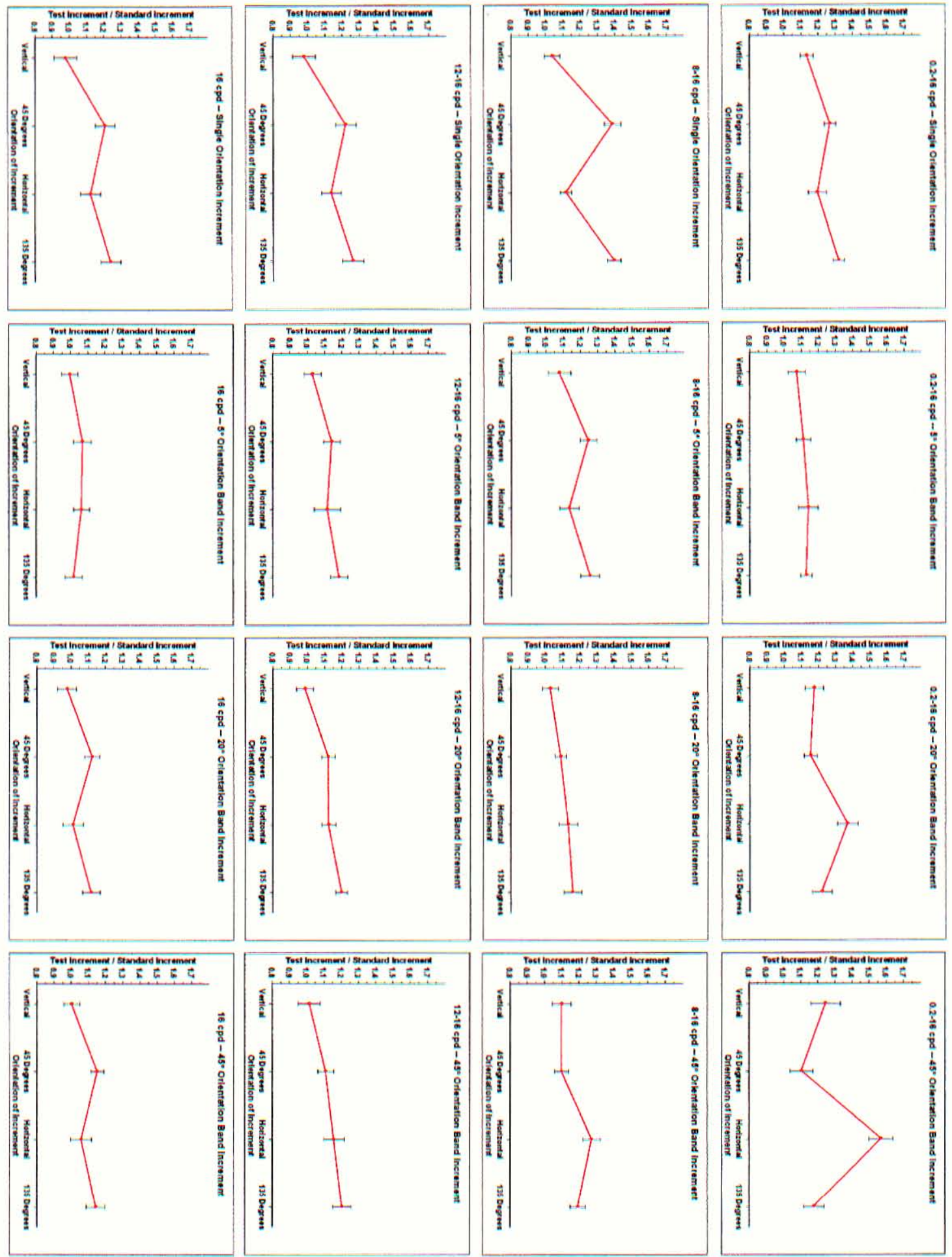
Figure 29. Graph matrix showing the results of the 16 different conditions investigated in Experiment 1. The layout of the graph matrix follows exactly the layout of the conditions in Table 1 . Specifically, the results from Condition 1 are plotted in the graph at the bottom left of the graph matrix, Condition 16 results plotted in the graph located at the top right of the matrix, etc. On the ordinate of each graph is the average ratio of the test increment scalar (i.e., the value participants indicated as being perceptually equivalent to that of the standard) to the standard increment scalar (error bars are \pm 1 SEM and represent the average within subjects SEM). Note that values greater than 1.0 indicate poor suprathreshold sensitivity. On the ordinate are the four test orientations. 

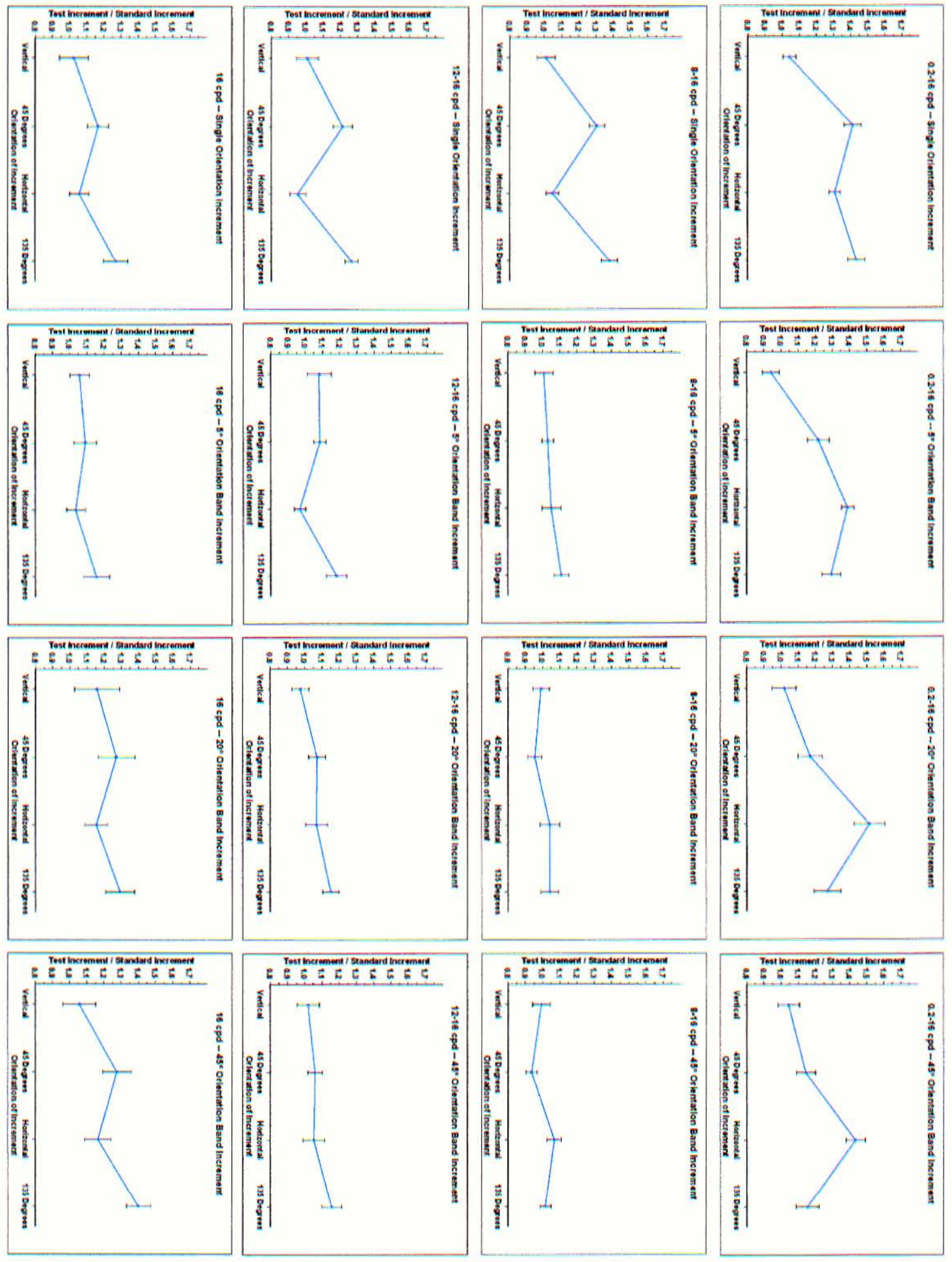
Figure 30. Graph matrix showing the results of the 16 different conditions investigated in Experiment 2. The spatial layout and plotting layout of the individual graphs is identical to Figure 29. 

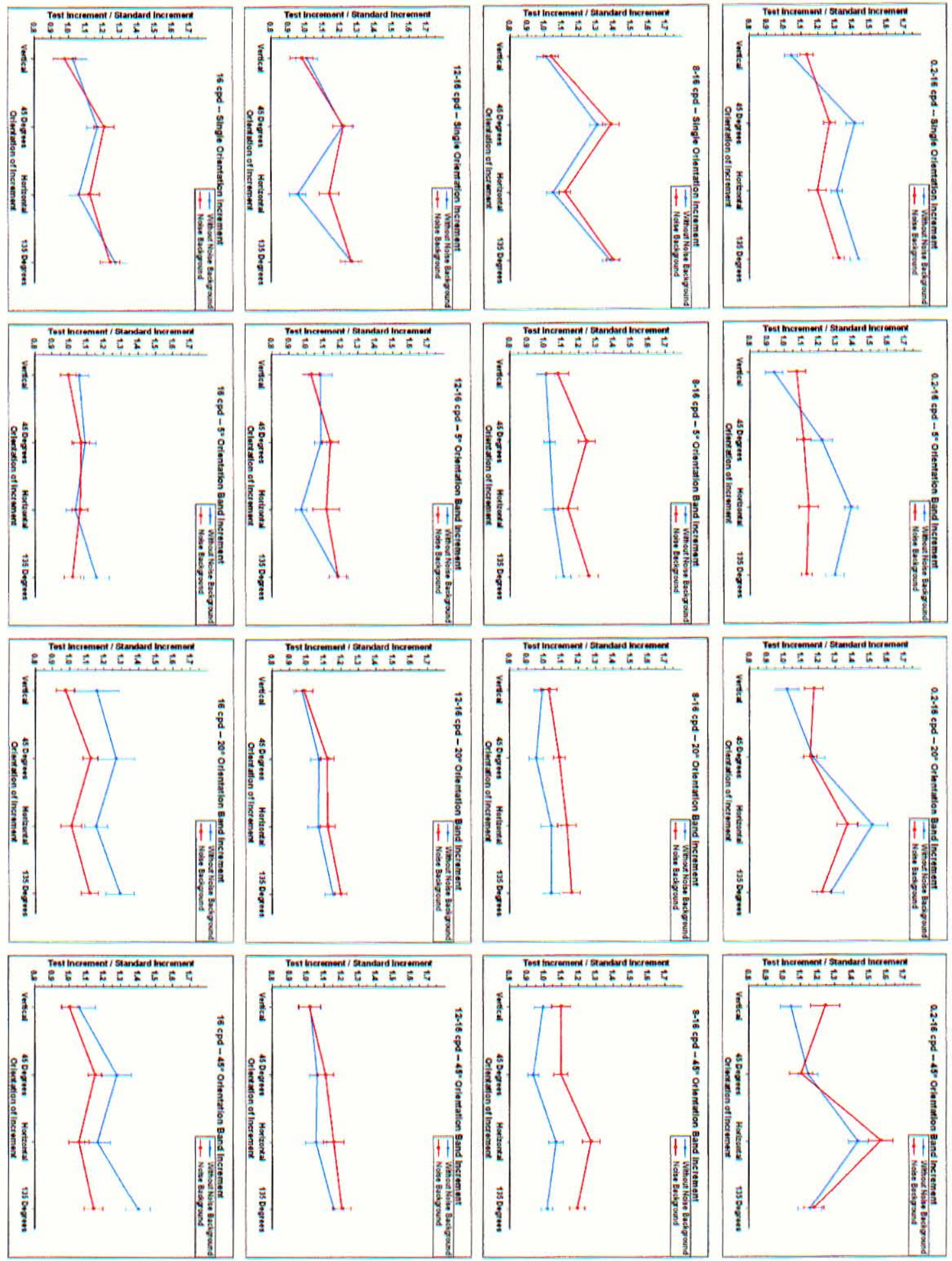
Figure 31. Graph matrix showing the results of the 16 different conditions investigated in Experiments 1 and 2. The spatial layout and plotting layout of the individual graphs is identical to Figures 29 and 30. Results from Experiment 1 are shown in red, results from Experiment 2 are shown in blue. 
A

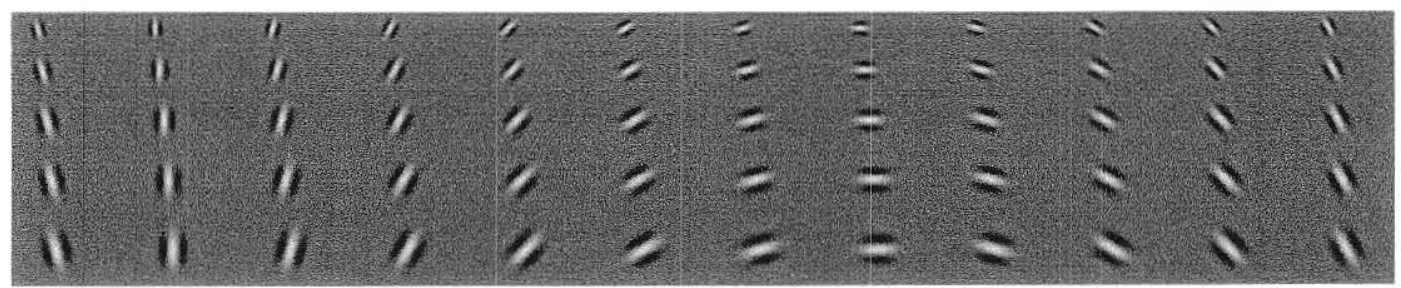

B
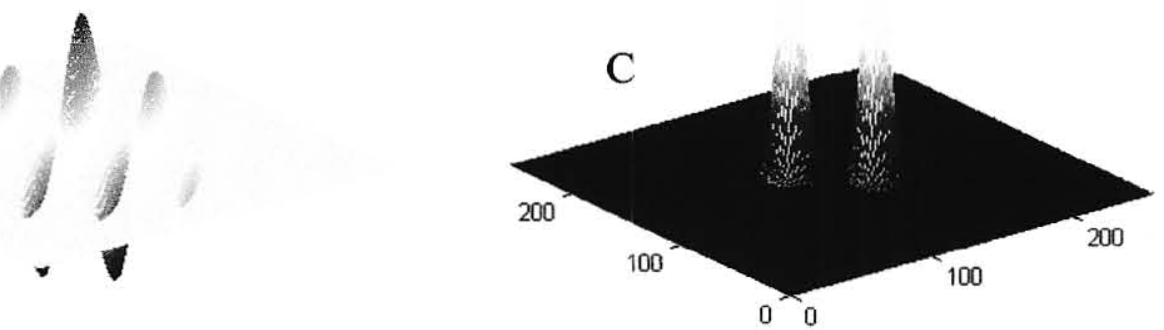

Figure 32. A) 2D spatial profiles of the full range of basis set filters described in the text.

Twelve different filter orientations will be generated (in steps of $15^{\circ}$ ) for five different spatial scales. Spatial scales will range from $0.29 \mathrm{cpd}$ to $1.33 \mathrm{cpd}$ (scale 1 ), $0.52 \mathrm{cpd}$ to $2.11 \mathrm{cpd}$ (scale 2), $1.25 \mathrm{cpd}$ to $4.48 \mathrm{cpd}$ (scale 3 ), $2.0 \mathrm{cpd}$ to $6.32 \mathrm{cpd}$ (scale 4 ), and $4.2 \mathrm{cpd}$ to 11.72cpd (scale 5, the highest scale allowed by the Nyquist limit for this imagery that will be filtered). B) 3D spatial profile for one of the filters in the proposed basis set. C) 3D profile for one of the filters in the proposed basis set, depicted in the Fourier domain. 


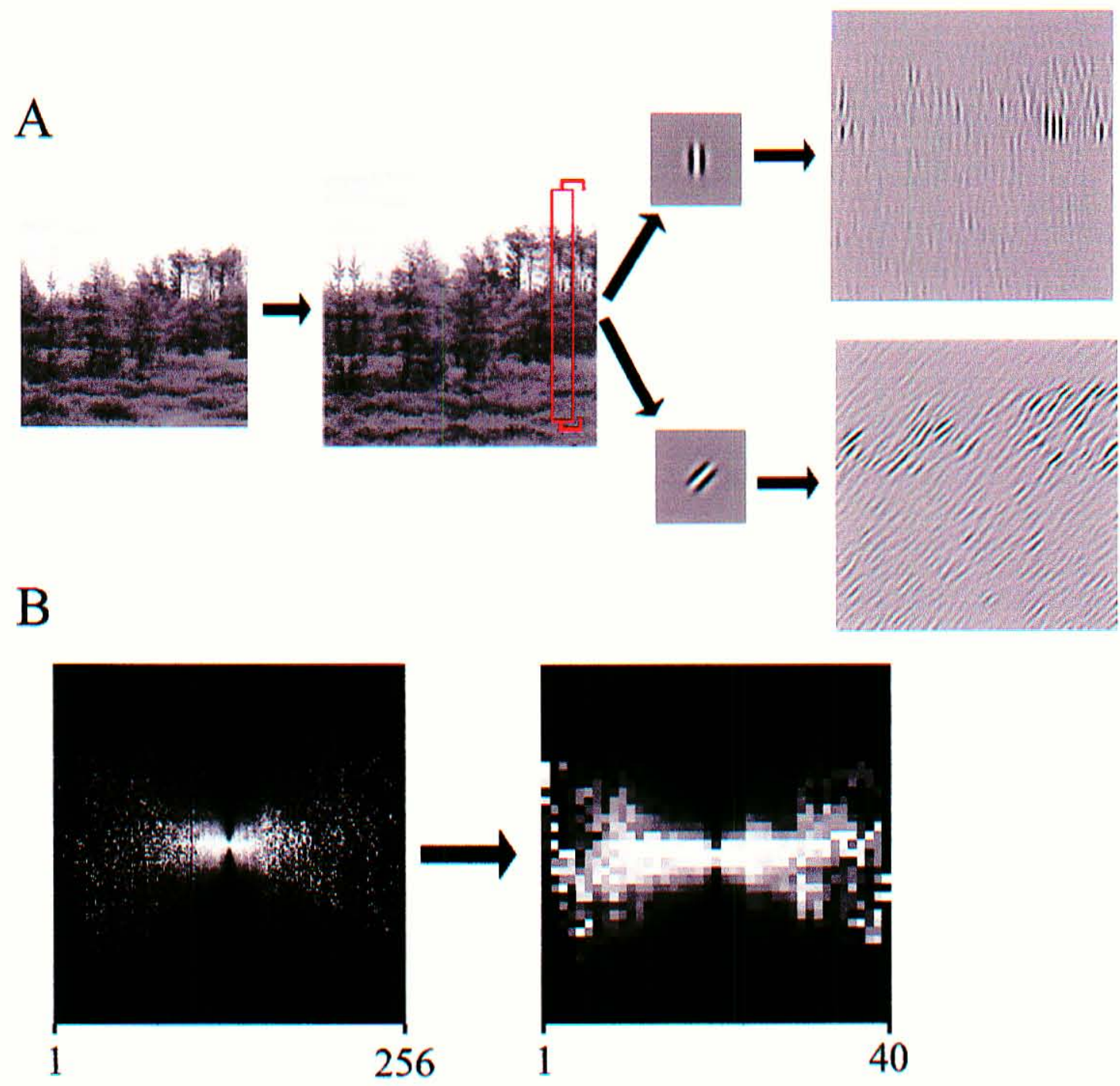

Figure 33. A) An illustration of the neural response simulation procedures proposed in the text. The first step involves mirroring the outer 40 pixels in either the $x$ or $y$ dimensions by 'flipping' those pixels to the outer edge of the image; note that this step increases the image size by 80 pixels in both the $x$ or $y$ dimensions. The resulting image is then filtered with one of each of the filters in the proposed basis set (vertical and $45^{\circ}$ are shown in the current figure). After the filtering procedure, the central $512 \times 512$ pixels are cropped form the filtered image and then normalized to 0 to 1 (depicted here). B) On the left is the full 2D joint histogram obtained from the two filtered images shown in (A), on the right is the same 2D joint histogram that has been down-sampled to a $40 \mathrm{x}$ 
40 pixel matrix. On the abscissa of every joint histogram is the full range of grayscale values (1-256) for the image filtered by the primary filter, on the ordinate is the range of grayscale values for the same image filtered with a secondary (or comparison) filter. The histogram itself is a plot of the number of co-occurrences between each of the grayscale values of the primary filtered image and the secondary filtered image. The more two grayscale values co-occurred (i.e., corresponding locations within the two filtered images), the brighter the that coordinate in the joint histogram. 


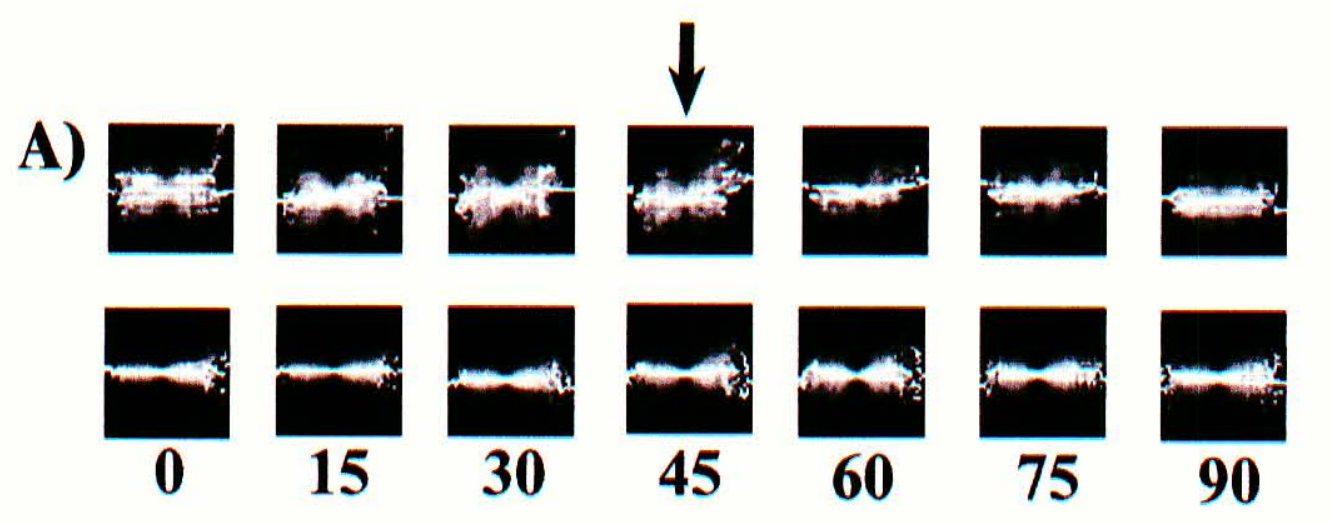

B)

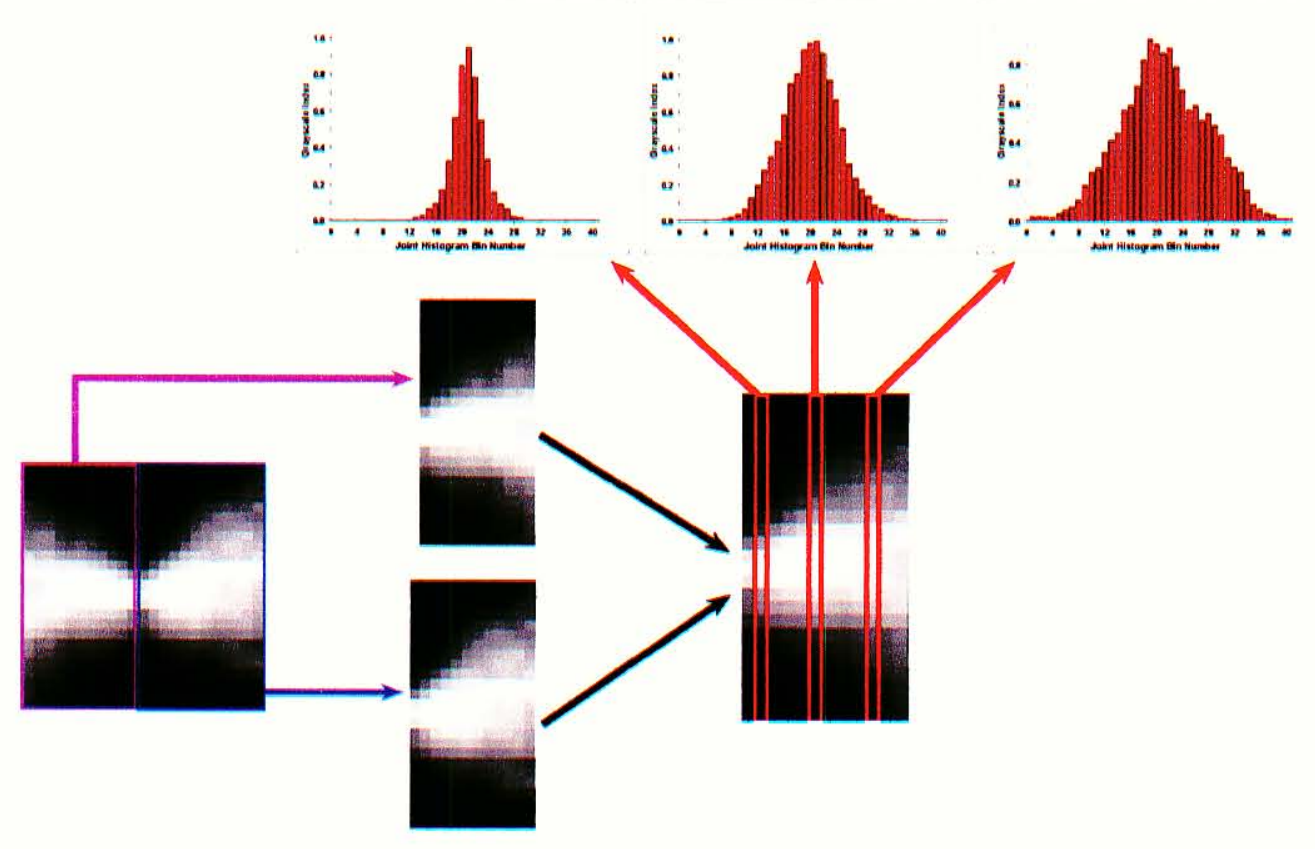

Figure 34. For A, the joint histograms were obtained from a set of filter response images where the primary filter scale was scale 3 , arrows indicate orientation of the primary filter which, for this example, matched the orientation of the content-biased image. Top-row, each of the joint histograms shown are between the primary filter response image and filter response images from 7 differently oriented secondary filters either one scale away (i.e. scale 2). Bottom row, joint histograms between the primary filter response image filter response images from 7 differently oriented secondary filters two scales away (i.e. scale 5). Note that the closer the secondary filters are in terms of orientation and scale, the more the 2D joint histograms show a filter response dependency. B) An example of 
a 2D joint histogram exhibiting the bow-tie shape, thereby indicating strong filter response dependency. This shape was quantified in the current study by flipping the left portion (flipped about the central column which plots the mean of the two filter response images) of the histogram over and averaging it with the right half, resulting in a $21 \times 41$ matrix. Excluding the first column, the standard deviation of each column was calculated, paired with its position on the abscissa (some number between 1 and 40) and then fit with a regression line. The slope of that regression line thus served as a quantified representation of the strength of the dependency between any two filter response image pairs. 


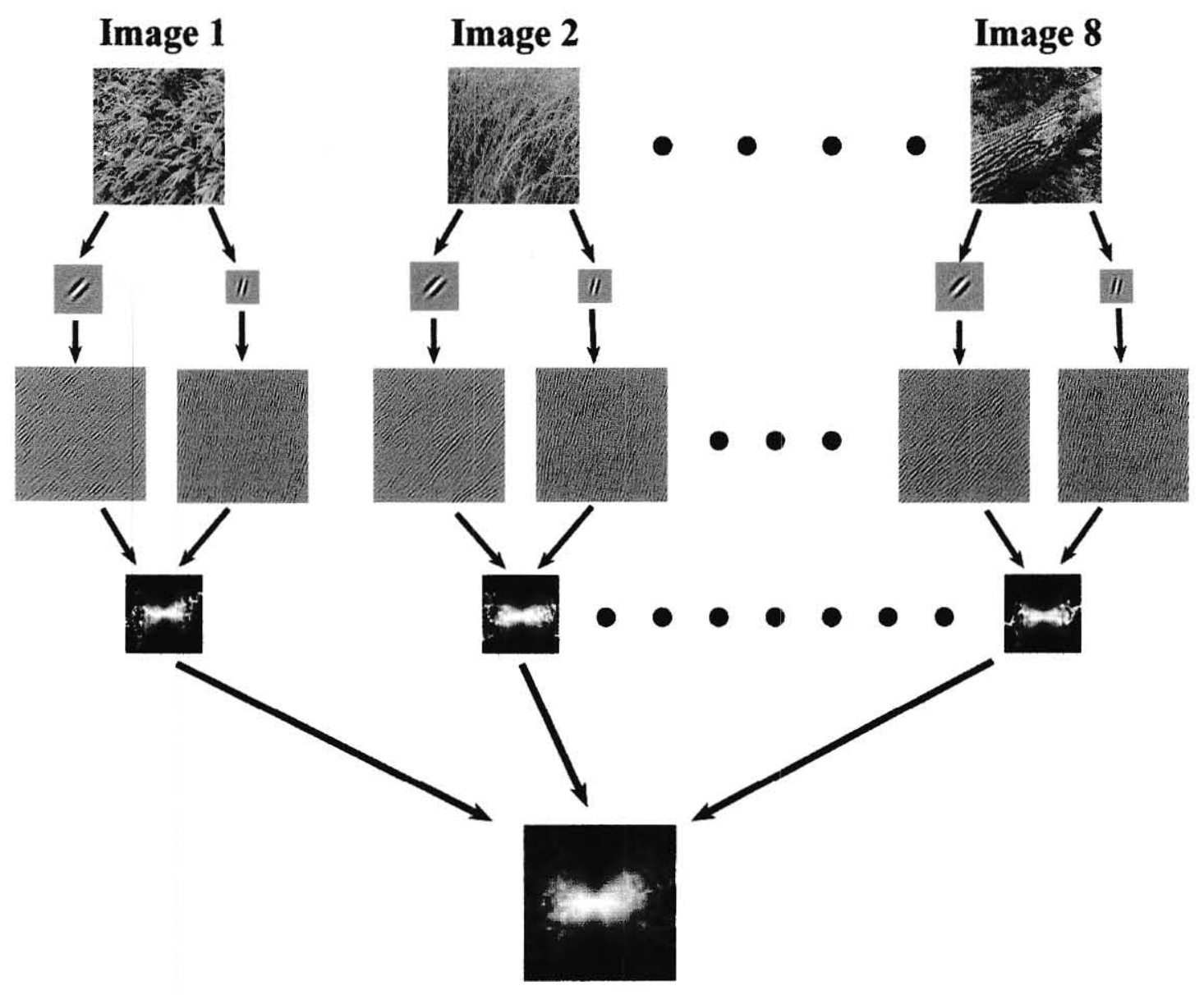

Figure 35. Illustration of the averaging procedure implemented in the analysis of the joint histograms obtained from the content-dependent effect 1 simulation. At the top are a few examples of natural scene images with a $45^{\circ}$ content bias (total of eight for each content bias set). Below the images are the primary filter with a peak response at scale 3 and $45^{\circ}$ oriented content and the secondary filter with a peak response at scale 4 and $15^{\circ}$ oriented content. Below the filters are the corresponding filter response images produced by the filters. The two filter response images were then used to create a joint histogram (shown below each filter response image pair). The eight joint histograms obtained from this pair of filters, for this set of images, were then averaged (refer to the text for further details). 


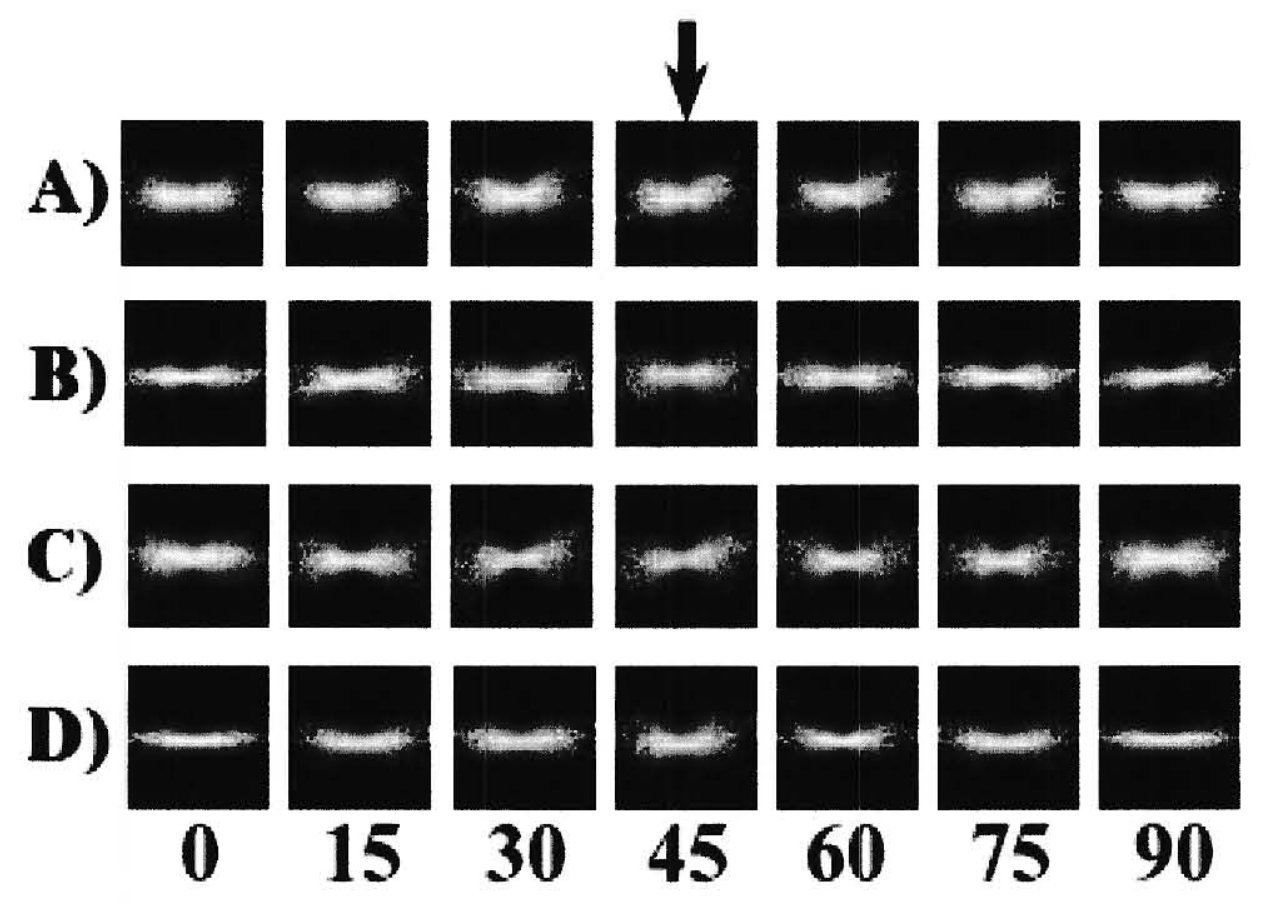

Figure 36. Averaged joint histograms obtained from comparing a primary filter (peak response at scale 3 and for content oriented at $45^{\circ}$ ) response images with secondary filter response images that were filtered by functions that had peak responses at the other four spatial scales, and for six different orientations. The arrow indicates the orientation of the primary filter. Rows A-D are the histograms from the comparisons with secondary filters that were selective for scales $1,2,4,5$ respectively. At the bottom of the figure is the orientation of the secondary filters. Notice that the strongest averaged response dependencies (prominent 'bow-tie' shape) were obtained when the orientation and scale of the secondary filter was more similar to that of the primary filter. For comparisons between the primary and secondary filter responses that were less similar with respect to spatial scale and orientation, the responses dependency was less prominent. 


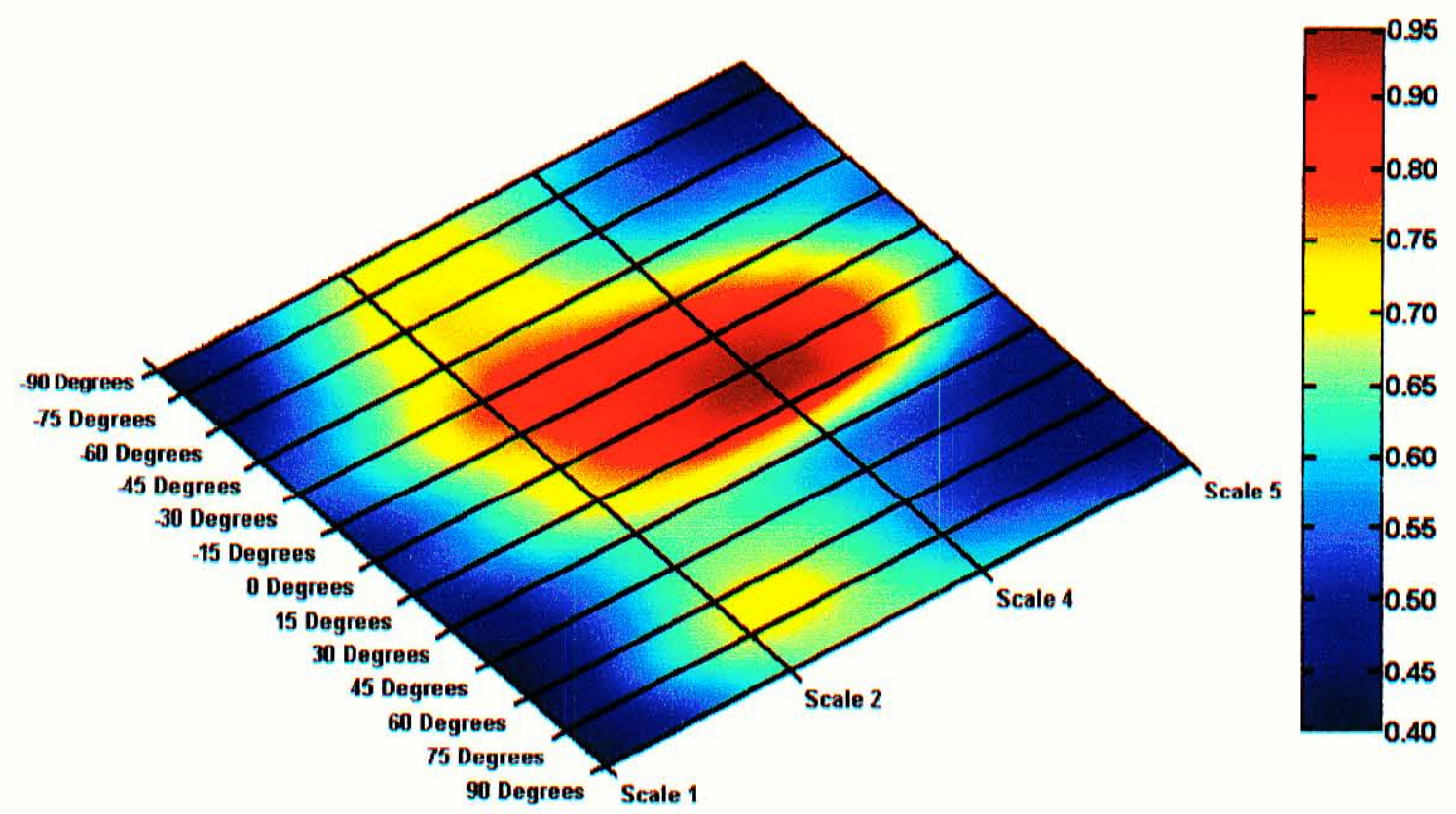

Figure 37. Plot of the 'weights' that were obtained by the methods described in the text for the content-dependent effect 1 . Note that this plot is very similar to what is depicted in Figure 36, only here it has been quantified. In Figure 36 the orientation of the primary filter was $45^{\circ}$ and the images from which those histograms were obtained also had a bias in content at that orientation. As mentioned in the text, the same comparisons were made for primary filters tuned to the orientations of the content biases in the other three image sets. Thus, there were three other versions of that which is shown in Figure 36. Here, all four of those versions (including all orientations of the secondary filters, instead of six) were aligned at the primary filter orientation and averaged. There are two important notes regarding this plot, first is that the data have been smoothed using the bicubic interpolation method, and second, that the color map has been scaled to match the range of weights obtained during the dependency analysis described in the text. On the $y$-axis is the full range of orientations of the secondary filters, with zero indicating that the orientation of the primary and secondary filter was identical. On the $x$-axis is the scale 
(spatial frequency range) to which the different secondary filters were selective. Notice the region of this plot with the highest values corresponds to secondary filters that were very similar to the primary filter with respect to scale and orientation selectivity (refer to the text for further details). 


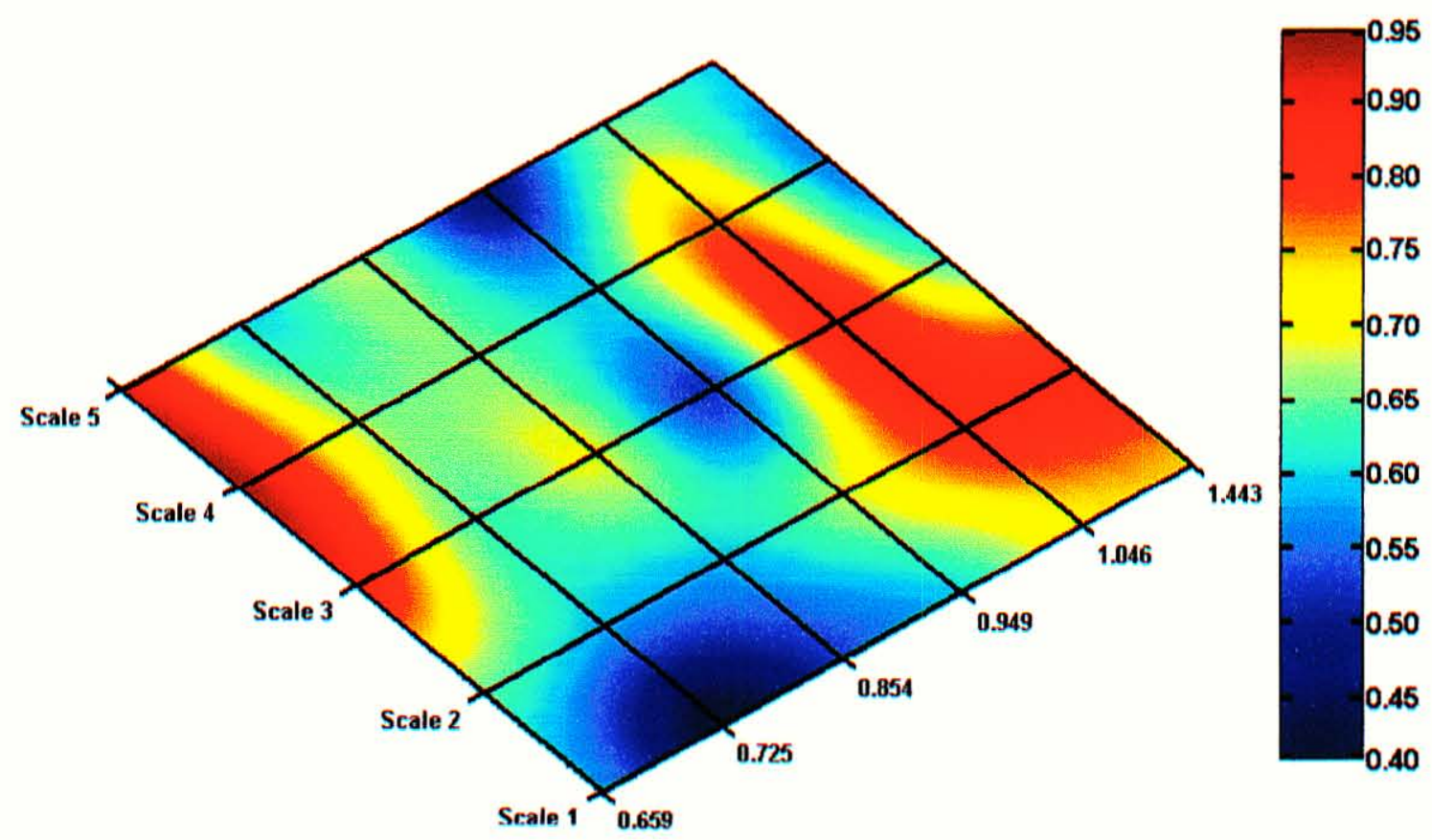

Figure 38. Plot of the 'weights' that were obtained by the methods described in the text for the content-dependent effect 2. Since the strength of the response dependencies between the filters selective for different orientations was irrelevant here, the weights were averaged across orientation within each of the five spatial scales. This averaging was carried out for the weights obtained within each slope image set. As with Figure 37, there are two important notes regarding this plot, first is that the data have been smoothed using the bicubic interpolation method, and second, that the color map has been scaled to match the range of weights obtained during the dependency analysis described in the text. On the $y$-axis is the full range of spatial scales (spatial frequency range) of the filters examined in the simulations. On the $x$-axis is the amplitude spectrum slope value associated with each of the six different image sets utilized in the content-dependent effect 2 simulation. Notice that the regions of this plot with the highest values correspond to the higher spatial scales for imagery that possessed very shallow amplitude 
spectrum slopes, and lower spatial scales when the imagery possessed amplitude spectrum slopes that were very steep. 


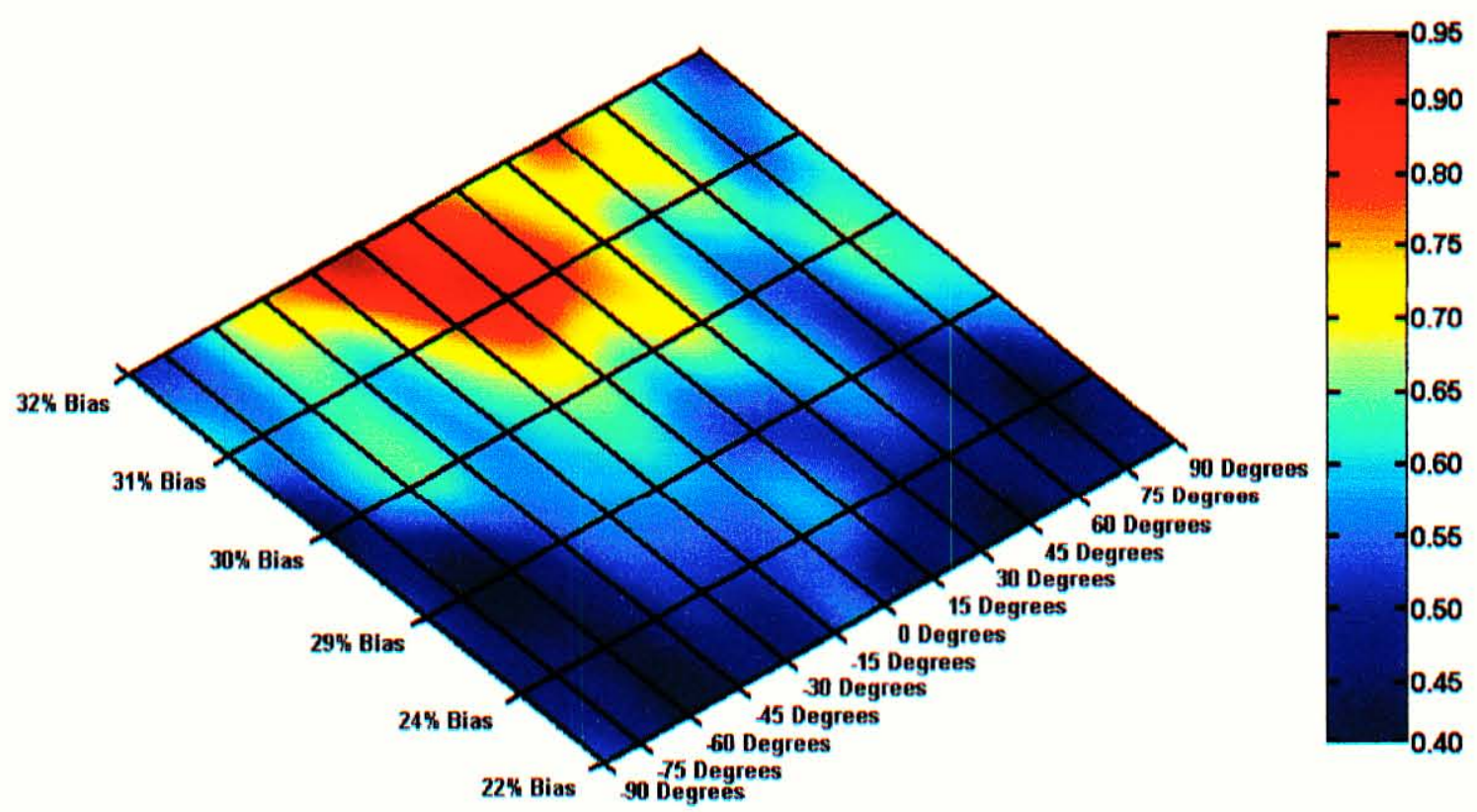

Figure 39. Plot of the 'weights' that were obtained by the methods described in the text for the content-dependent effect 3. As with Figures 37 and 38, there are two important notes regarding this plot, first is that the data have been smoothed using the bicubic interpolation method, and second, that the color map has been scaled to match the range of weights obtained during the dependency analysis described in the text. On the $x$-axis is the full range of orientations of the secondary filters, with zero indicating that the orientation of the primary and secondary filter was identical (which was always horizontal for this analysis). On the $y$-axis is the percentage of horizontal content bias (refer to the text for details regarding how this percentage was calculated) for each of the six images utilized in the content-dependent effect 3 simulation. If one were to select any given $y$-axis grid line, the method in which those weights were plotted was identical to that depicted in Figure 37, only here the weights have been averaged across scale and only horizontal biased images have been subjected to analysis. Notice that as the content bias at horizontal increases, the response dependencies between the horizontally oriented 
primary filters and all other secondary filters increase and are localized near horizontal (i.e., 0 degrees). 


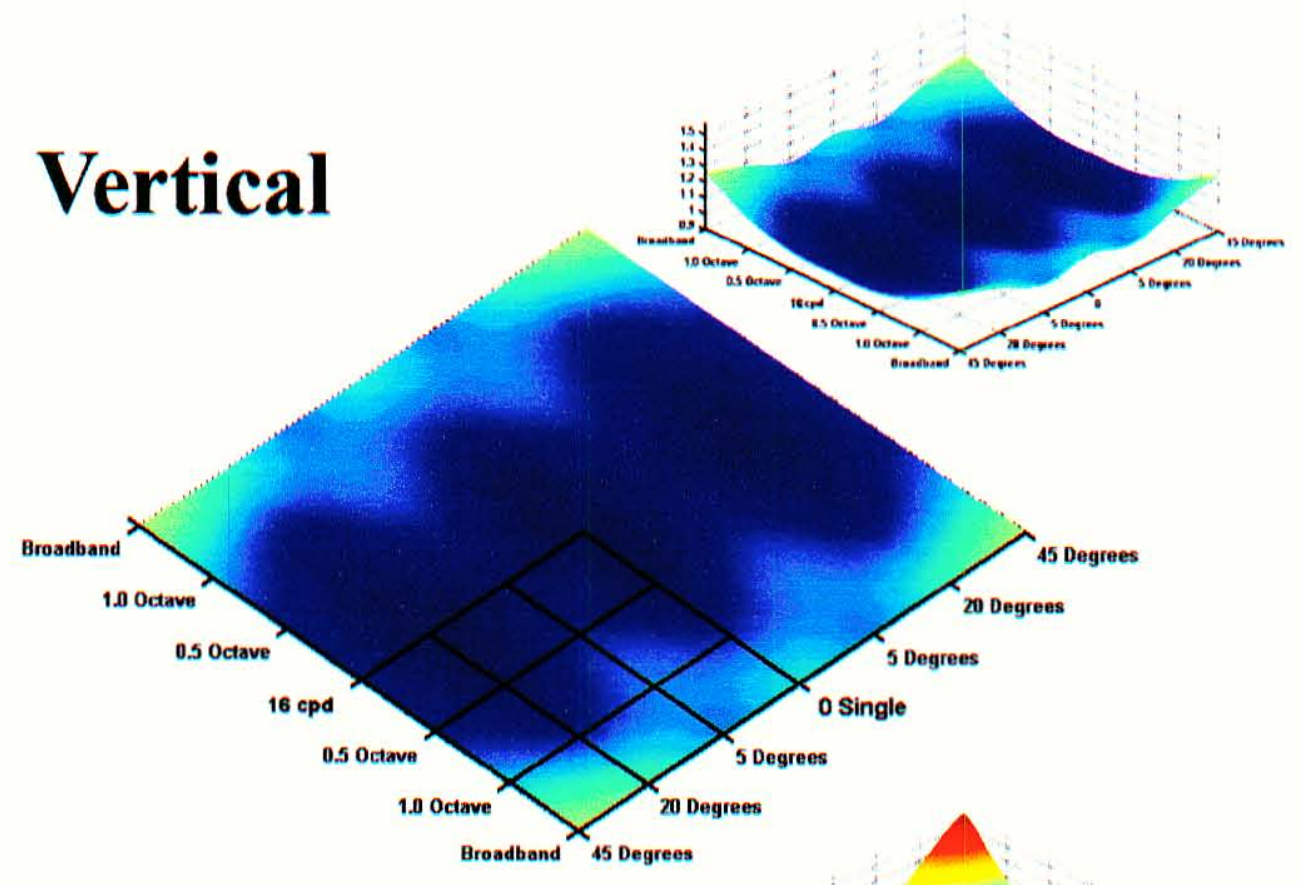

\section{Horizontal}
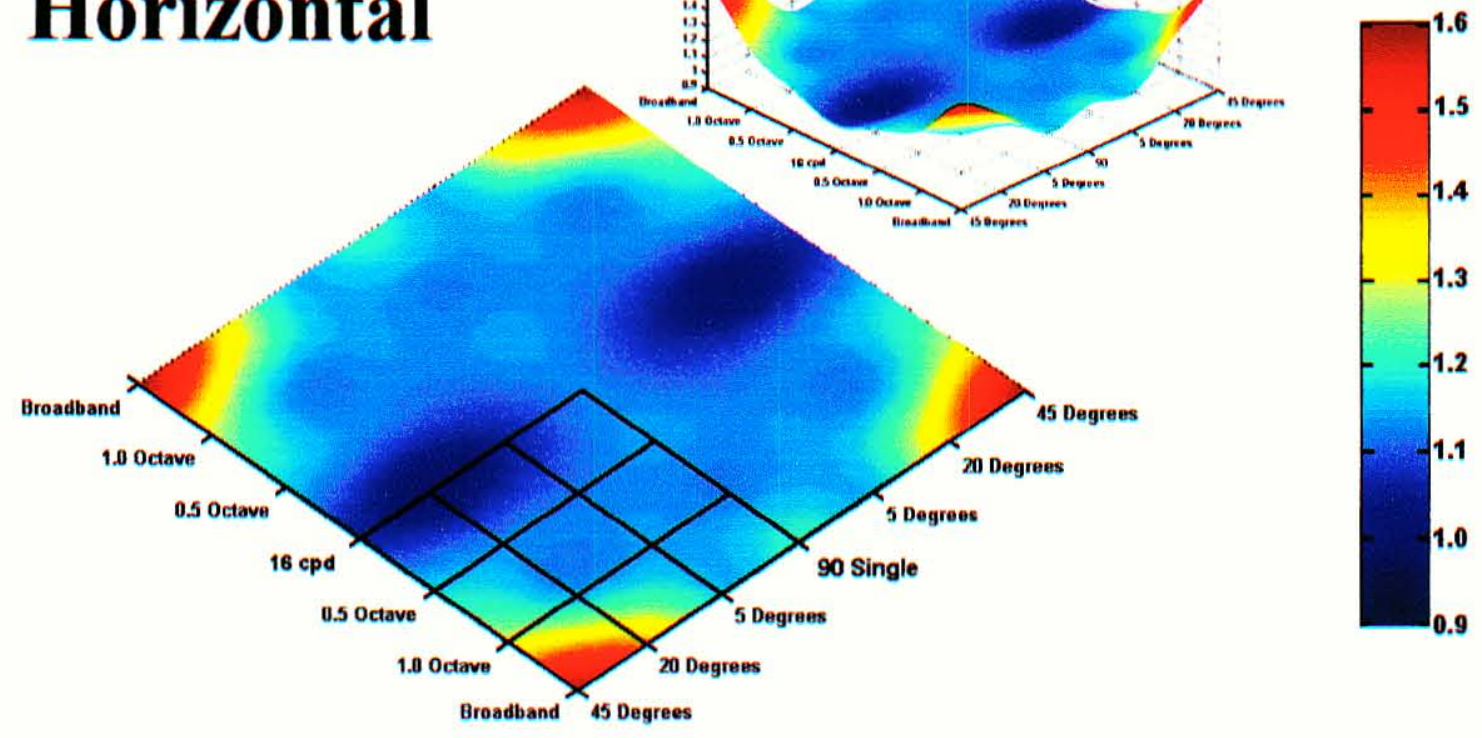

Figure 40a. Weighting indices generated from the data obtained in Experiment 1. A) The weighting indices for vertical and horizontal suprathreshold sensitivity. B) The weighting indices for $45^{\circ}$ and $135^{\circ}$ oblique orientation suprathreshold sensitivity. For both $\mathrm{A}$ and $\mathrm{B}$, two views of each index are shown, note that the color map has been scaled to fit the range of weights obtained from both experiments. Additionally, the 
original indices have been smoothed with bicubic interpolation. For the top view graphs of the inherent weighting indices, a grid has been drawn where each crossing point indicates the 'location' of each of the 16 conditions (refer to the text for further details). 

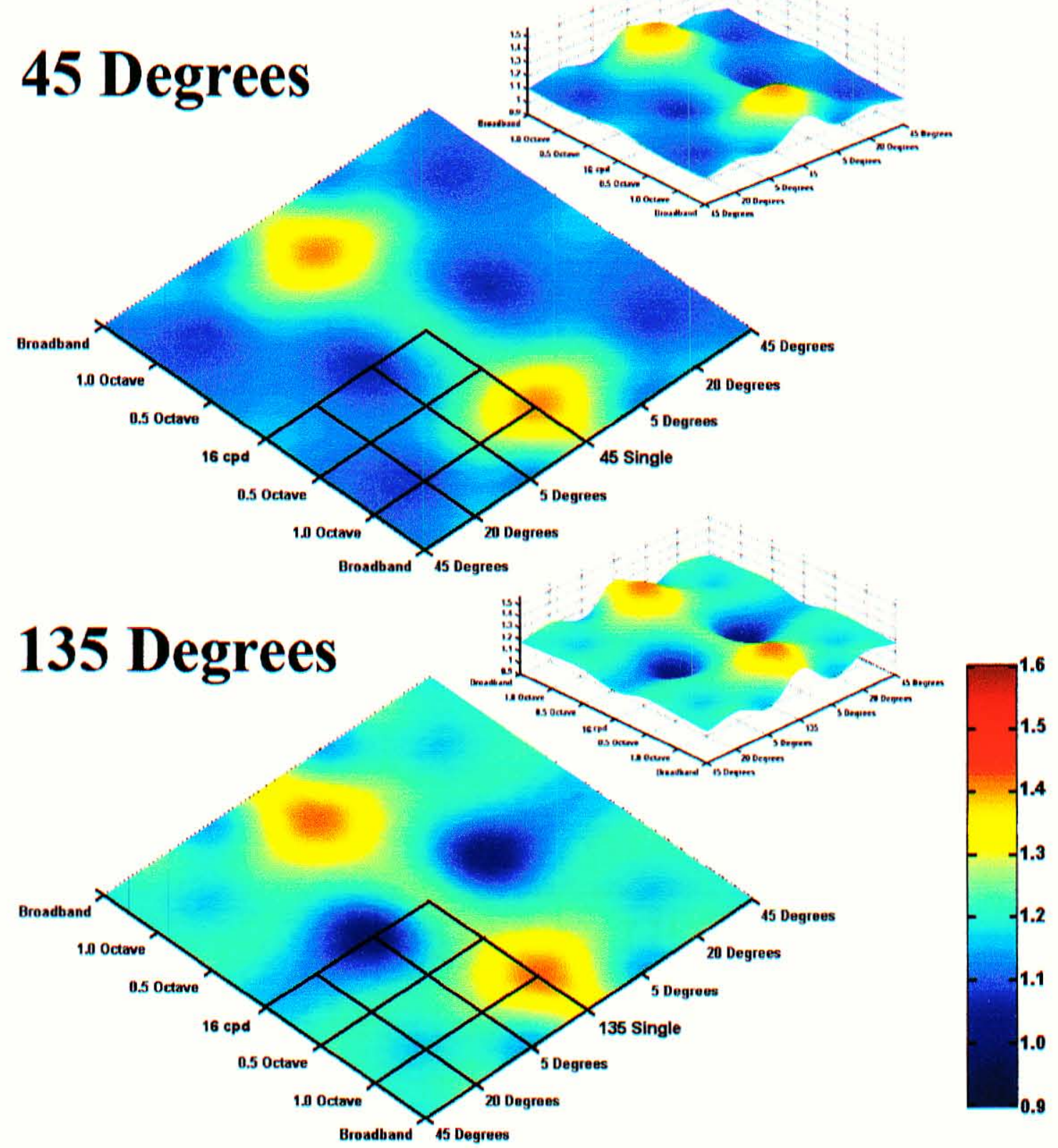

Figure 40b. Weighting indices generated from the data obtained in Experiment 1. A) The weighting indices for vertical and horizontal suprathreshold sensitivity. B) The weighting indices for $45^{\circ}$ and $135^{\circ}$ oblique orientation suprathreshold sensitivity. For both $\mathrm{A}$ and $\mathrm{B}$, two views of each index are shown, note that the color map has been 
scaled to fit the range of weights obtained from both experiments. Additionally, the original indices have been smoothed with bicubic interpolation. For the top view graphs of the inherent weighting indices, a grid has been drawn where each crossing point indicates the 'location' of each of the 16 conditions (refer to the text for further details). 


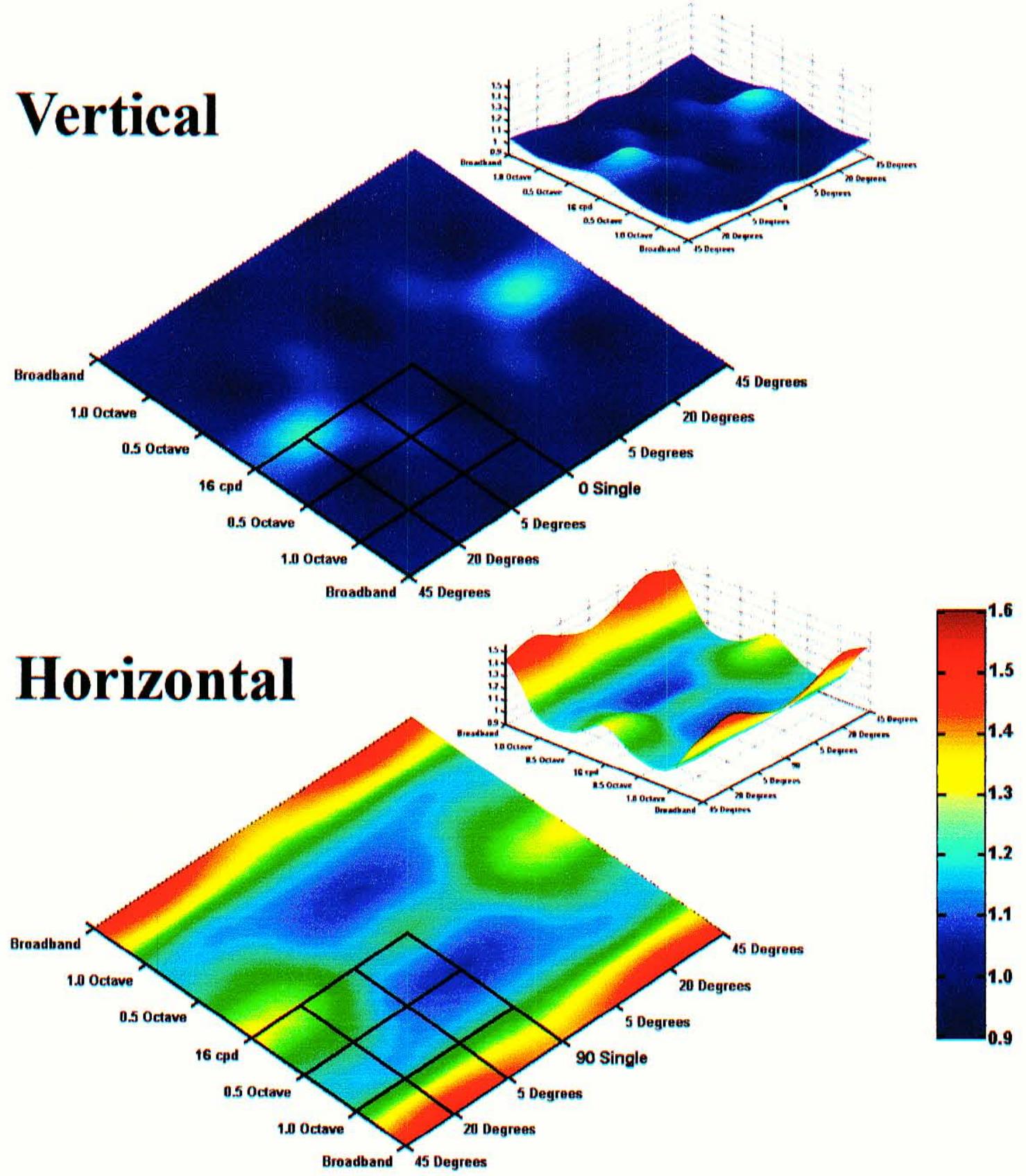

Figure 41a. Weighting indices generated from the data obtained in Experiment 2. A) The weighting indices for vertical and horizontal suprathreshold sensitivity. B) The weighting indices for $45^{\circ}$ and $135^{\circ}$ oblique orientation suprathreshold sensitivity. For both $\mathrm{A}$ and $\mathrm{B}$, two views of each index are shown, note that the color map has been scaled to fit the range of weights obtained from both experiments. Additionally, the 
original indices have been smoothed with bicubic interpolation. For each of the top view graphs of the inherent weighting indices, a grid has been drawn, where each crossing point indicates the 'location' of the data point from each of the 16 conditions (refer to the text for further details). 


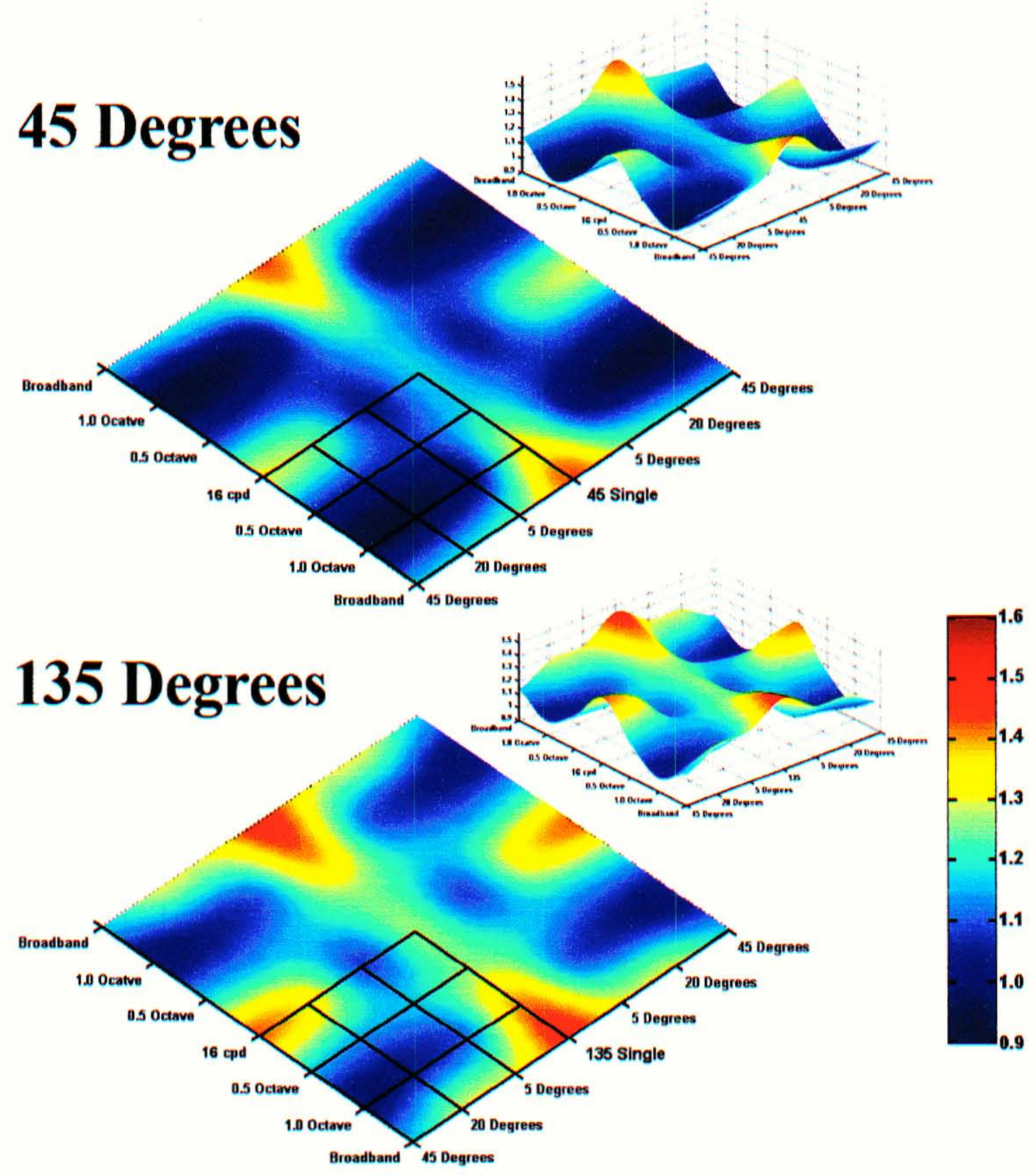

Figure 41b. Weighting indices generated from the data obtained in Experiment 2. A) The weighting indices for vertical and horizontal suprathreshold sensitivity. B) The weighting indices for $45^{\circ}$ and $135^{\circ}$ oblique orientation suprathreshold sensitivity. For both $\mathrm{A}$ and $\mathrm{B}$, two views of each index are shown, note that the color map has been scaled to fit the range of weights obtained from both experiments. Additionally, the 
original indices have been smoothed with bicubic interpolation. For each of the top view graphs of the inherent weighting indices, a grid has been drawn, where each crossing point indicates the 'location' of the data point from each of the 16 conditions (refer to the text for further details). 
A)
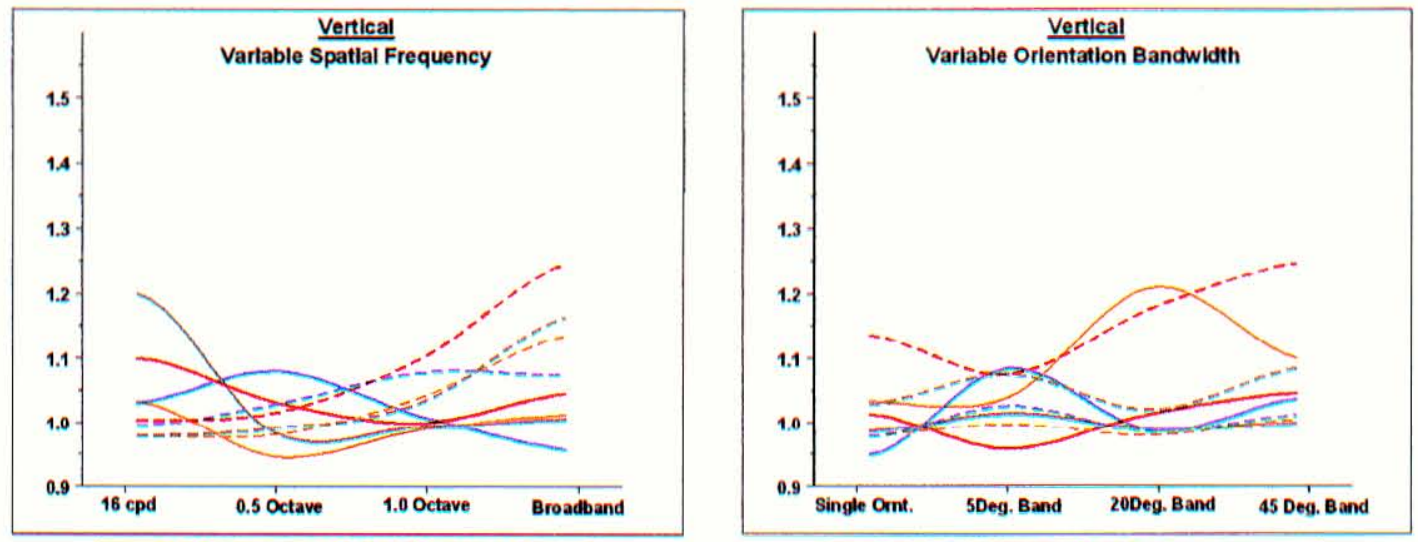

- No-Background: Single Orientation

- No-Background: 5 Degree Bandwidth

- No-Background: 20 Degree Bandwidth

- No-Background: 45 Degree Bandwidth

--- Background: Single Orientation

--- Background: 5 Degree Bandwidth

-.- Background: 20 Degree Bandwidth

--- Background: 45 Degree Bandwidth

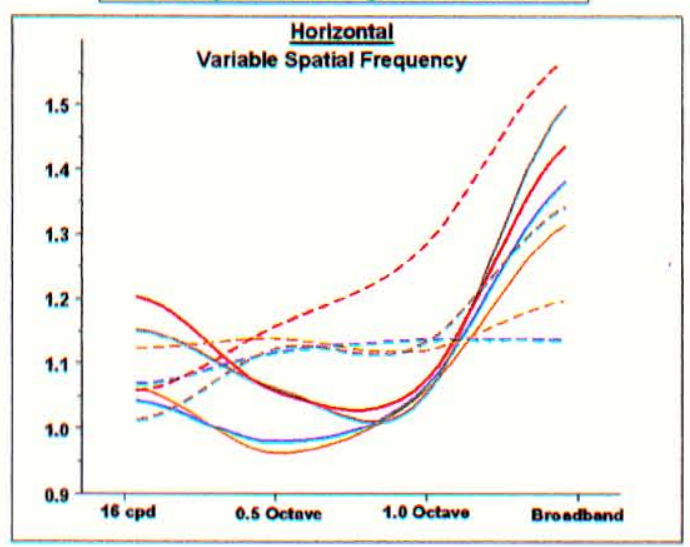

- No-Background: Single Frequency

- No-Background: 0.5 Octave Bandwidth

- No-Background: 1.0 Octave Bandwidth

- No-Background: Broadband

-.- Background: Single Frequency

-.. Background: 0.5 Octave Bandwidth

-- Background: 1.0 Octave Bandwidth

-- Background: Broadband

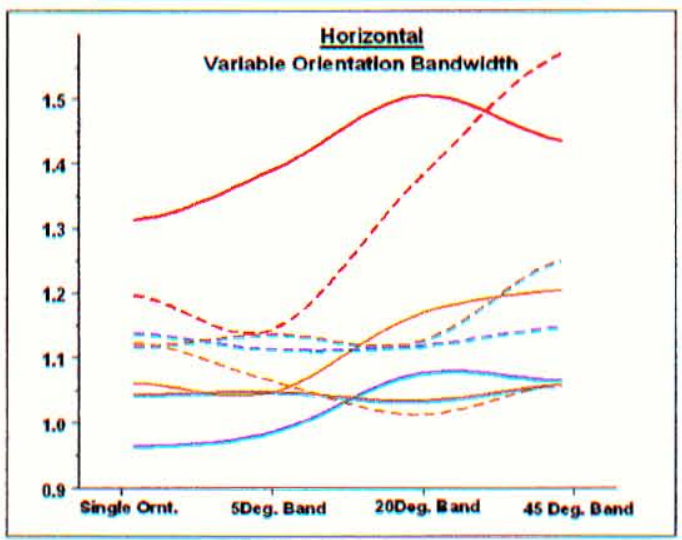


B)

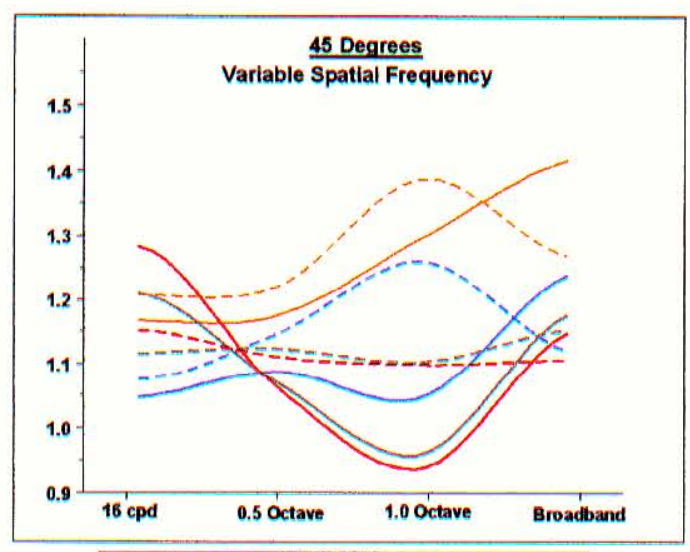

- No-Background: Single Orientation

- No-Background: 5 Degree Bandwidth

- No-Background: 20 Degree Bandwidth

- No-Background: 45 Degree Bandwidth

-.- Background: Single Orientation

--- Background: 5 Degree Bandwidth

-... Background: 20 Degree Bandwidth

-- Background: 45 Degree Bandwidth

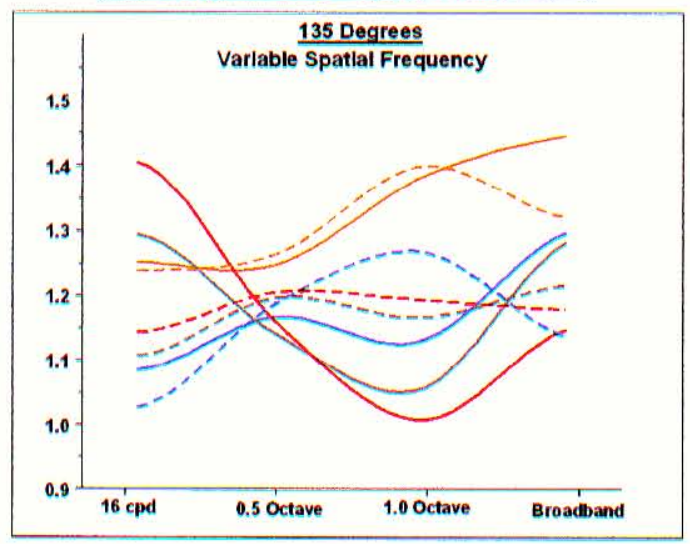

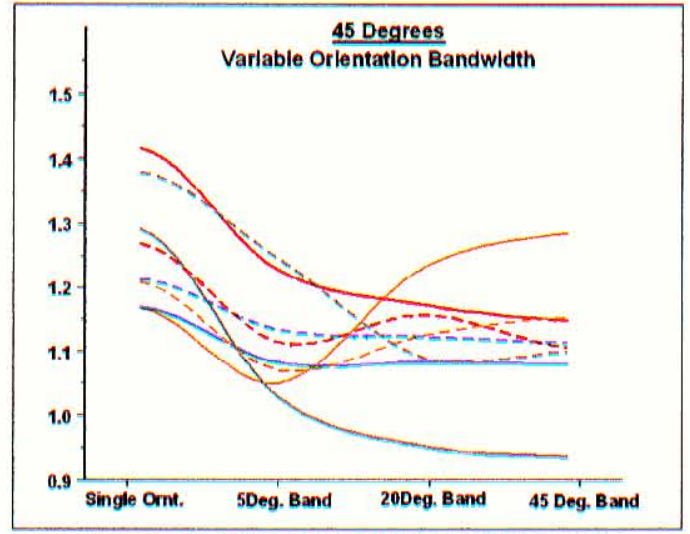

- No-Background: Single Frequency

- No-Background: 0.5 Octave Bandwidth

- No-Background: 1.0 Octave Bandwidth

- No-Background: Broadband

- - Background: Single Frequency

-... Background: 0.5 Octave Bandwidth

-.- Background: 1.0 Octave Bandwidth

-.- Background: Broadband

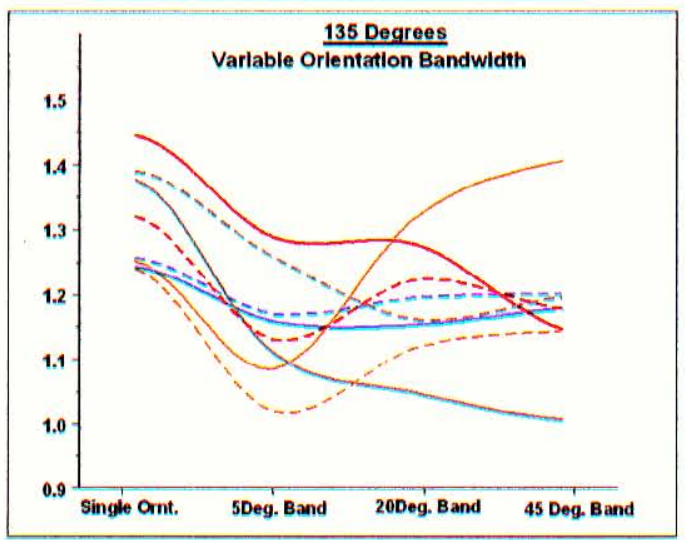

Figure 42. Vector samples obtained from the different grid lines of the weighting indices generated from the data obtained in Experiment 1 and 2. A) The vector samples from the weighting indices for vertical and horizontal shown in Figures 41a and 42a. B) The vector samples from the weighting indices for $45^{\circ}$ and $135^{\circ}$ oblique orientations shown in Figures $41 \mathrm{~b}$ and $42 \mathrm{~b}$. For each orientation, two graphs are provided. The graphs on the left plot the vectors from the four different orientation bandwidth conditions where spatial frequency was allowed to vary (i.e., the grid lines extending from the $x$-axis in 
Figures 41 and 42). The graphs on the rights plot the vectors from the four different spatial frequency bandwidth conditions where orientation bandwidth was allowed to vary (i.e., the grid lines extending from the $y$-axis in Figures 41 and 42). On the ordinate of each graph is the weight associated with each of those conditions (high values indicate poor sensitivity). On the abscissa of each graph is the bandwidth of the parameter that was allowed to vary. Within each plot are four vectors from each experiment, the solid curves are from the no-background experiment and the dashed curves are from the noise background experiment. 


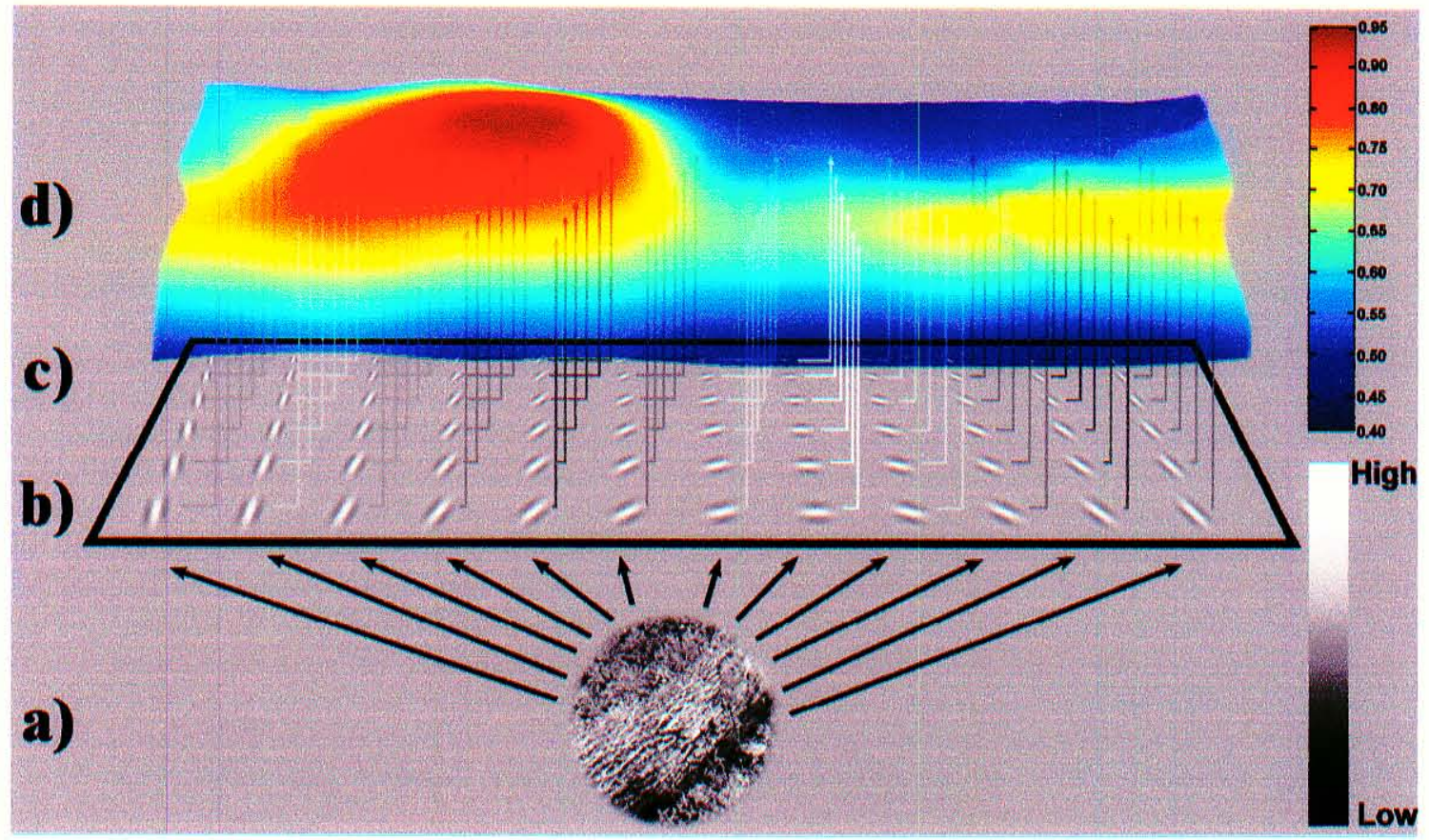

Figure 43. Schematic representation of the normalization pool associated neural units selective for a given orientation (refer to the text for further details regarding the layout and interpretation of this figure). The color mapping is identical to that of Figure 37 as are the weights associated with the top color-bar. The grayscale color bar provides neural numerical bias scale, where lower numbers biases are represented by darker shades of gray and higher numerical biases are represented by the brighter shades of gray. Note that this shading scheme has been applied to the output arrows extending up from each representative neural unit for orientation (represented along the front) and spatial frequency (represented along the sides). Thus, the horizontal $>$ vertical $>$ obliques numerical bias can be represented. 


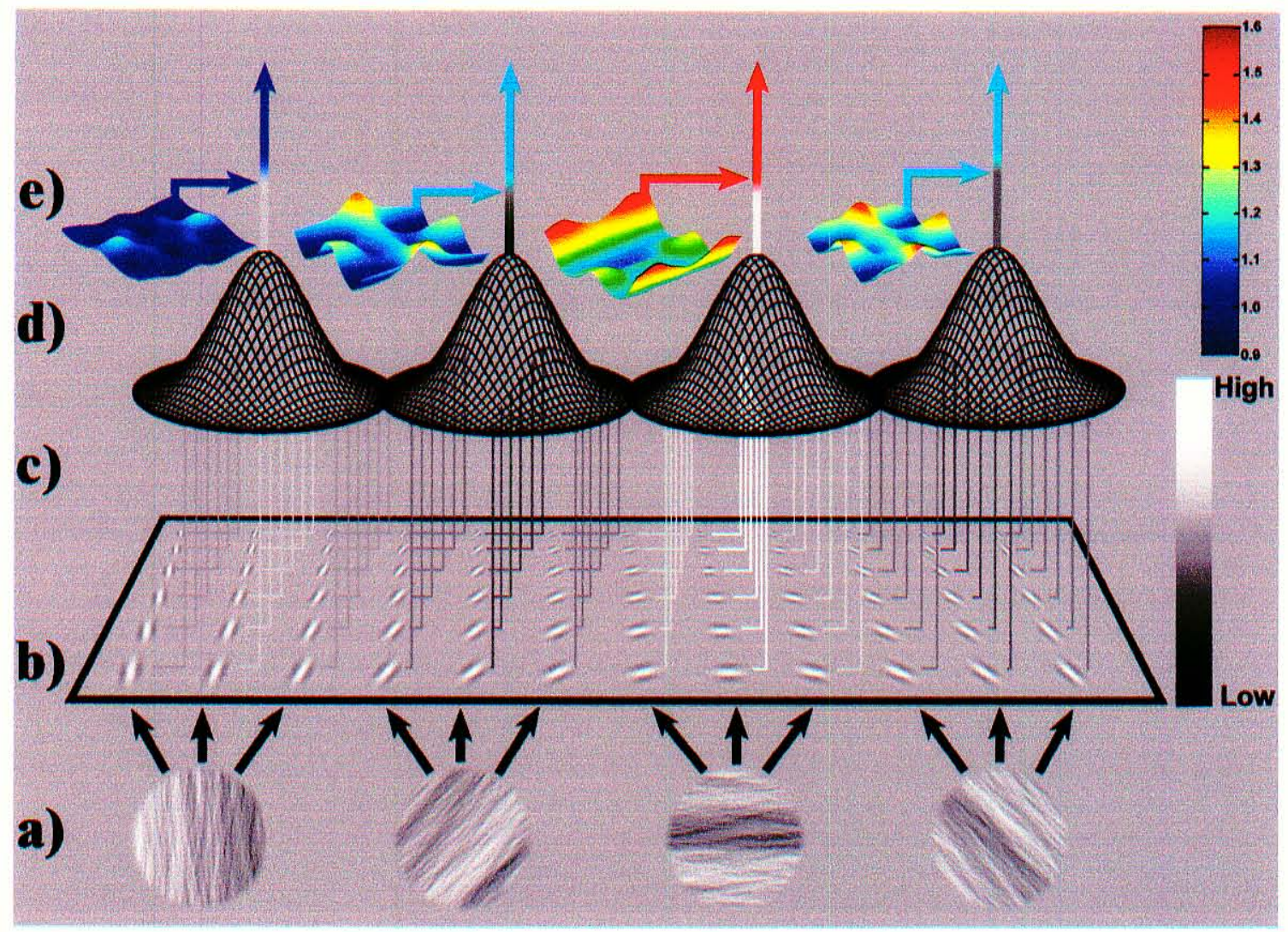

Figure 44. Schematic representation of how the inherent weighting indices are utilized to update the output signals as a function of changing structure (resulting from the different increment extents) contained in the input image (refer to the text for further details regarding the layout and interpretation of this figure). The color mapping of the indices is identical to that of Figures 40 and 41, as are the weights associated with the top colorbar. The grayscale color bar provides neural numerical bias scale, where lower numbers biases are represented by darker shades of gray and higher numerical biases are represented by the brighter shades of gray. 


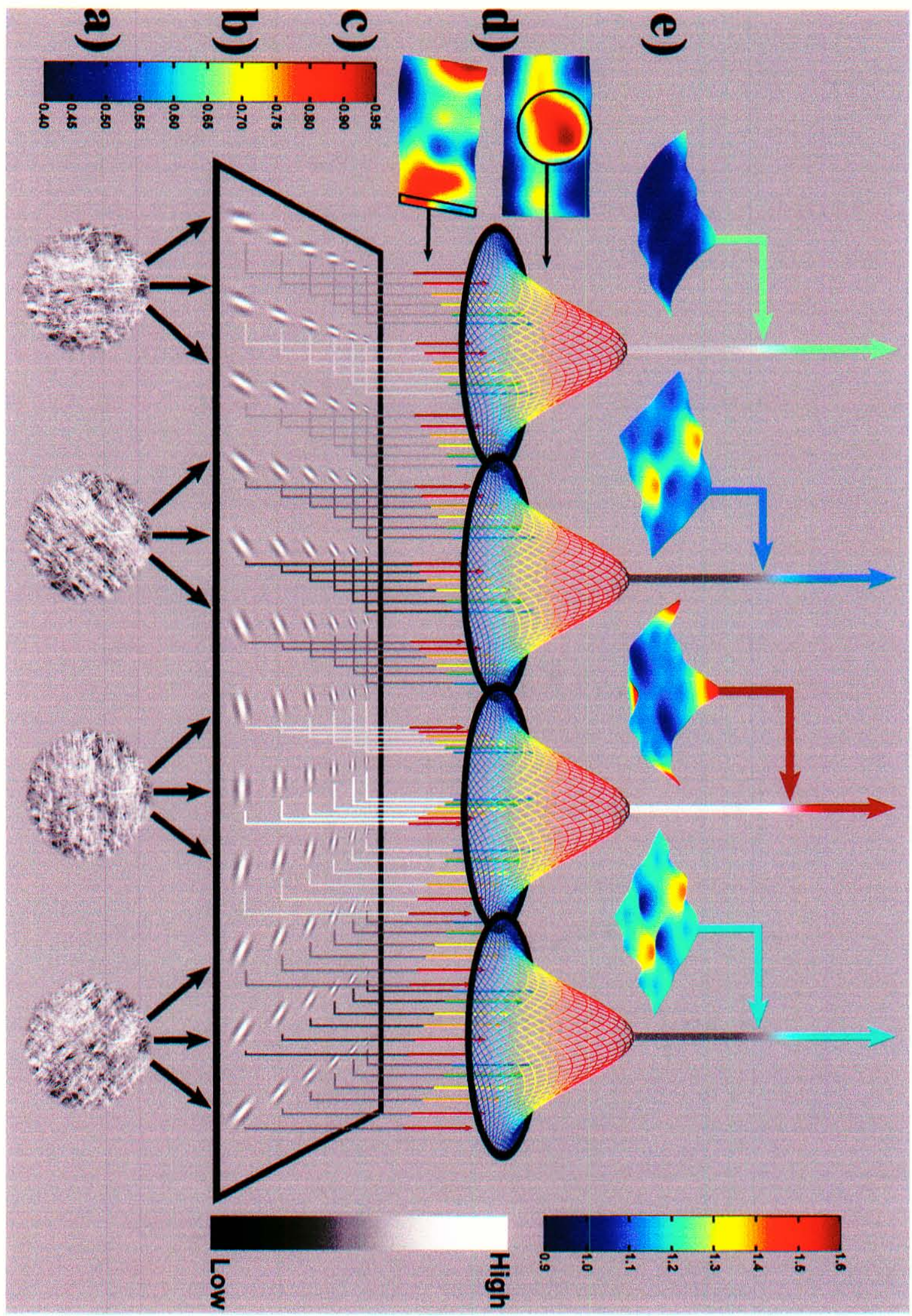


Figure 45. Full schematic representation of the proposed striate normalization model (refer to the text for further details regarding the layout and interpretation of this figure). The color mapping of the indices is identical to that of Figures 40 and 41, as are the weights associated with the top color-bar. The grayscale color bar provides neural numerical bias scale, where lower numbers biases are represented by darker shades of gray and higher numerical biases are represented by the brighter shades of gray. 


\section{REFERENCES}

Annis, R.C. \& Frost, B. (1973). Human visual ecology and orientation anisotropies in acuity. Science, 182, 729-731.

Anzai, A., Bearse, A., Jr., Freeman, R.D., \& Cai, D. (1995). Contrast coding by cells in the cat's striate cortex: Monocular vs. binocular detection. Visual Neuroscience, 12, 77-93.

Appelle, S. (1972). Perception and discrimination as a function of stimulus orientation: The oblique effect in man and animals. Psychological Bulletin, 78, 266-278.

Atick, J.J. \& Redlich, A.N. (1992). What does the retina know about natural scenes? Neural Computation, 4, 196-210.

Baddeley, R.J. \& Hancock, P.J.B. (1991). A statistical analysis of natural images matches psychophysically derived orientation tuning curves. Proceedings of the Royal Society of London B, 246, 219-223.

Baddeley, R.J. (1997). The correlational structure of natural images and the calibration of spatial representations. Cognitive Science, 21, 351-372.

Blakemore, C. \& Campbell, F.W. (1969). On the existence of neurons in the human visual system selectively sensitive to the orientation and size of retinal images. Journal of Physiology, 203, 237-260. 
Blakemore, C. \& Cooper, G.F. (1970). Development of brain depends on visual environment. Nature, 228, 477-481.

Blakemore, C. \& Nachmias, J. (1971). The orientation specificity of two visual aftereffects. Journal of Physiology, London, 213, 157-174.

Bonds, A.B. (1989). Role of inhibition in the specification of orientation selectivity of cells in the cat striate cortex. Visual Neuroscience, 2, 41-55.

Bonds, A.B. (1991). Temporal dynamics of contrast gain in single cells of the cat striate cortex. Visual Neuroscience, 6, 239-255.

Bracewell, R.N. (2000). The fourier transform and its applications. McGraw-Hill Companies, Boston.

Burton, G.J. \& Moorhead, I.R. (1987). Color and spatial structure in natural scenes. Applied Optics, 26, 157-170.

Carandini, M. \& Heeger, D.J. (1994). Summation and division in primate visual cortex. Science, 264, 1333-1336.

Campbell, F.W., Kulikowski, J.J., \& Levinson, J. (1966). The effect of orientation on the visual resolution of gratings. Journal of Physiology (London), 187, 427-436.

Chapman, B., Stryker, M.P., \& Bonhoeffer, T. (1996). Development of orientation preference maps in ferret primary visual cortex. The Journal of Neuroscience, 16, 6443-6453.

Craven, B.J. (1993). Orientation dependence of human line-length judgments matches statistical structure in real-world scenes. Proceedings of the Royal Society of London, 253, 101-106. 
Coppola, D.M., Purves, H.R., McCoy, A.N., \& Purves, D. (1998). The distribution of oriented contours in the real world. Proceedings of the National Academy of Sciences USA, 95, 4002-4006.

Coppola, D.M., White, L.E., Fitzpatrick, D., \& Purves, D. (1998). Unequal representation of cardinal and oblique contours in ferret visual cortex. Proceedings of the National Academy of Sciences USA, 95, 2621-2623.

De Valois, R.L., Albrecht, D.G., \& Thorell, L.G. (1982). Spatial frequency selectivity of cells in macaque visual cortex. Vision Research, 22, 545-559.

De Valois, R.L., Yund, E.W., \& Hepler, N. (1982). The orientation and direction selectivity of cells in macaque visual cortex. Vision Research, 22, 531-544.

Essock, E.A. (1980). The oblique effect of stimulus identification considered with respect to two classes of oblique effects. Perception, 9, 37-46.

Essock, E.A., Krebs, W.K., \& Prather, J.R. (1997). Superior sensitivity for tactile stimuli oriented proximally-distally on the finger: Implications for mixed class 1 and class 2 anisotropies. Journal of Experimental Psychology: Human Perception and Performance, 23, 515-527.

Essock, E.A., DeFord, J.K., Hansen, B.C. \& Sinai, M.J. (2003). Oblique stimuli are seen best (not worst!) in naturalistic broad-band stimuli: A horizontal effect, Vision Research, 43, 1329-1335.

Falmagne, J.C. (1986). Psychophysical measurement and theory. In K.R. Boff, L. Kaufman, and J.P. Thomas (Eds.) Handbook of perception and human performance. Vol. 1: Sensory processes and perception (pp. 1-1- 1-65). John Wiley and Sons, New York. 
Field, D.J. (1987). Relations between the statistics of natural images and the response properties of cortical cells. Journal of the Optical Society of America A, 4, 23792394.

Field, D.J. \& Brady, N. (1997). Visual sensitivity, blur and the sources of variability in the amplitude spectra of natural scenes. Vision Research, 37, 33673383.

Furmanski, C.S. \& Engel, S.A. (2000). An oblique effect in human primary visual cortex. Nature Neuroscience, 3, 535-536.

Geisler, W.S. \& Albrecht, D.G. (1992). Cortical-neurons-isolation of contrast gain control. Vision Research, 32, 1409-1410.

Hancock, P.J.B., Baddeley, R.J., \& Smith, L.S. (1992). The principle components of natural images. Network: Computation in Neural Systems, 3, 61-70.

Hansen, B.C. \& Essock, E.A. (2004a). A horizontal bias in human visual processing of orientation and its correspondence to the structural components of natural scenes. Journal of Vision (In Press).

Hansen, B.C. \& Essock, E.A. (2004b). Influence of scale and orientation on the visual perception of natural scenes. Visual Cognition (In Press).

Hansen, B.C., Essock, E.A., Zheng, Y., \& DeFord, J.K. (2003). Perceptual anisotropies in visual processing and their relation to natural image statistics. Network: Computation in Neural Systems, 14, 501-526.

Heeger, D.J. (1992a). Normalization of cell responses in cat striate cortex. Visual Neuroscience, 9, 181-197. 
Held, R., Thorn, F., McLellan, J., Grice, K., \& Gwiazda, J. (2000). Does early astigmatism contribute to the oblique effect and account for its difference between chinese and caucasians? Proceedings of the VIII International Conference on Myopia, Conference on Myopia 2000, Inc.

Hirsh, H.V.B. \& Spinelli, D.N. (1970). Visual experience modifies distribution of horizontally and vertically oriented receptive fields in cats. Science, 168, 869-871.

Howe, C.Q. \& Purves, D. (2002). Range statistics can explain the anomalous perception of length. Proceedings of the National Academy of Sciences, 99, 13184-13188.

Hubel, D.H. \& Wiesel, T.N. (1970). Period of susceptibility to physiological effects of unilateral eye closure in kittens. Journal of Physiology-London, 206, 419-436.

Jastrow, J. (1893). On the judgement of angles and positions of lines. American Journal of Psychology, 5, 214-248.

Keil, M.S. \& Cristóbal, G. (2000). Separating the chaff from the wheat: Possible origins of the oblique effect. Journal of the Optical Society of America A, 17, 697 710.

Knill, D.C., Field, D.J., \& Kersten, D. (1990). Human discrimination of fractal images. Journal of the Optical Society of America, 7, 1113-1123.

Lashley, K.S. (1938). The mechanisms of vision: Xv. preliminary studies of the rat's capacity for detail vision. Journal of General Psychology, 18, 123-193.

Li, B.W., Peterson, M.R., \& Freeman, R.D. (2003). The oblique effect: A neural basis in the visual cortex. Journal of Neurophysiology (In press).

Maffei, L. \& Campbell, F.W. (1970). Neurophysiological localization of the vertical and horizontal visual coordinates in man. Science, 187, 386-387. 
Mansfield, R.J.W. (1974). Neural basis of orientation perception in primate vision. Science, 186, 1133-1135.

Mansfield, R.J.W. \& Ronner, S.P. (1978). Orientation anisotropy in monkey visual cortex. Brian Research, 149, 229-234.

Marčelja, S. (1980). Mathematical description of the responses of simple cortical cells. Journal of the Optical Society of America, 70, 1297-1300.

Oliva, A. \& Torralba, A. (2001). Modeling the shape of the scene: A holistic representation of the spatial envelope. International Journal of Computer Vision, 42, 145-175.

Orban, G.A. \& Kennedy, H. (1980). Evidence for meridional anisotropies in orientation selectivity of visual cortical neurons. Archives Internationales de Physiologie et de Biochimie, 88, 13-14.

Pantle, A. \& Sekuler, R. (1968). Size-detecting mechanisms in human vision. Science, $162,1146-1148$.

Párraga, C.A. \& Tolhurst, D.J. (2000). The effect of contrast randomization on the discrimination of changes in the slopes of the amplitude spectra of natural scenes. Perception, 29, 1101-1116.

Párraga, C.A., Troscianko, T., \& Tolhurst, D.J. (2002). Spatiochromatic properties of natural images and human vision. Current Biology, 12, 483-487.

Rose, D. \& Blakemore, C. (1974). An analysis of orientation selectivity in the cat's visual cortex. Experimental Brain Research, 20, 1-17.

Schwartz, O. \& Simoncelli, E.P. (2001). Natural signal statistics and sensory gain control. Nature Neuroscience, 4, 819-825. 
Sengpiel, F., Stawinski, P., \& Bonhoeffer, T. (1999). Influence of experience on orientation maps in cat visual cortex. Nature Neuroscience, 2, 727-732.

Shapley, R. \& Lennie, P. (1985). Spatial frequency analysis in the visual system. Annual Review of Neuroscience, 8, 547-583.

Simoncelli, E.P., Freeman, W.T., Adelson, E.H., \& Heeger, D.J. (1992). Shiftable mulitscale transforms. IEEE Transactions in Information Theory, 38, 587-607.

Simoncelli, E.P. (1999). Modeling the joint statistics of images in the wavelet domain. Proceedings of the SPIE, 3813, 819-825.

Simoncelli, E.P. \& Olshausen, B.A. (2001). Natural image statistics and neural representation. Annual Review of Neuroscience, 24, 1193-1216.

Sokol, S., Moskowitz, A., \& Hansen, V. (1989). Evoked potential and preferential looking correlates of the oblique effect in 3-month-old infants. Documenta Opthalmologica, 71, 321-328.

Switkes, E., Mayer, M.J., \& Sloan, J.A. (1978). Spatial frequency analysis of the visual environment: Anisotropy and the carpentered environment hypothesis. Vision Research, 18, 1393-1399.

Tadmor, Y. \& Tolhurst, D.J. (1994). Discrimination of changes in the secondorder statistics of natural and synthetic images. Vision Research, 34, 541-554.

Tiao, Y-C. \& Blakemore, C. (1976). Functional organization in the visual cortex of the golden hamster. Journal of Comparative Neurology, 168, 459-482.

Thomson, M.G.A. (2001a). Sensory coding and the second spectra of natural signals. Physical Review Letters, 86, 2901-2904. 
Thomson, M.G.A. (2001b). Beats, kurtosis and visual coding. Network: Computation in Neural Systems, 12, 271-287.

Thomson, M.G.A. \& Foster, D.H. (1997). Role of second- and third-order statistics in the discriminability of natural images. Journal of the Optical Society of America A, 14, 2081-2090.

Tolhurst, D.J. \& Tadmor, Y. (1997). Band-limited contrast in natural images explains the detectability of changes in the amplitude spectra. Vision Research, 37, 3203-3215.

Tolhurst, D.J. \& Tadmor, Y. (2000). Discrimination of spectrally blended natural images: Optimization of the human visual system for encoding natural images. Perception, 29, 1087-1100.

Tolhurst, D.J., Tadmor, Y., \& Chao, T. (1992). Amplitude spectra of natural images. Ophthalmic and Physiological Optics, 12, 229-232.

Torralba, A. \& Oliva, A. (2003). Statistics of natural image categories. Network: Computation in Neural Systems, 14, 391-412.

van der Schaaf, A. \& van Hateren, J.H. (1996). Modeling the power spectra of natural images: Statistics and Information. Vision Research, 36, 2759-2770.

van Hateren, J.H. \& van der Schaaf, A. (1998). Independent component filters of natural images compared with simple cells in primary visual cortex. Proceedings of the Royal society of London: $B, 265,359-366$.

Wainwright, M.J., Schwartz, O. \& Simoncelli, E.P. (2001). Natural image statistics and 
divisive normalization: modeling nonlinearities and adaptation in cortical neurons In R. Rao, B. Olshausen and M. Lewicki (Eds.) Probabilistic Models of the Brain: Perception and Neural Function, Cambridge, MA: MIT Press.

Webster, M.A. \& Miyahara, E. (1997). Contrast adaptation on the spatial structure of natural images. Journal of the Optical Society of America A, 14, 2355-2366.

Wilson, H.R. (1991). Psychophysical models of spatial vision and hyperacuity. Spatial Vision, 10, 64-86.

Wilson, H.R. \& Humanski, R. (1993). Spatial frequency adaptation and contrast gain control. Vision Research, 33, 1133-1149.

Yoshida, S., Iwahara, S., \& Nagamura, N. (1975). The effect of stimulus orientation on the visual evoked potential in human subjects. Electroencephalography and Clinical Neurophysiology, 39, 53-57.

Yu, H-B. \& Shou, T-D. (2000). The oblique effect revealed by optical imaging in primary visual cortex of cats. Acta Physiologica Sinica, 52, 431-434.

Zemen, V., Gutowski, W., \& Horton, T. (1983). Orientational anisotropy in the human visual system: An evoked potential and psychophysical study. International Journal of Neuroscience, 19, 259-286. 


\begin{abstract}
APPENDIX
The following two figures are graph matrices showing the results from the 16 different conditions investigated in Experiment 1 (first matrix) and Experiment 2 (second matrix) for each of the nine participants. The layout of the graph matrix follows exactly the layout of the conditions in Table 1. Specifically, individuals results from Condition $l$ are plotted in the graph at the bottom left of the graph matrix, Condition 16 individual results are plotted in the graph located at the top right of the matrix, etc. On the ordinate of each graph is the average ratio of the test increment scalar (i.e., the value participants indicated as being perceptually equivalent to that of the standard) to the standard increment scalar (error bars are \pm 1 SEM and represent the average within subjects SEM). Note that values greater than 1.0 indicate poor suprathreshold sensitivity. On the ordinate are the four test orientations.
\end{abstract}



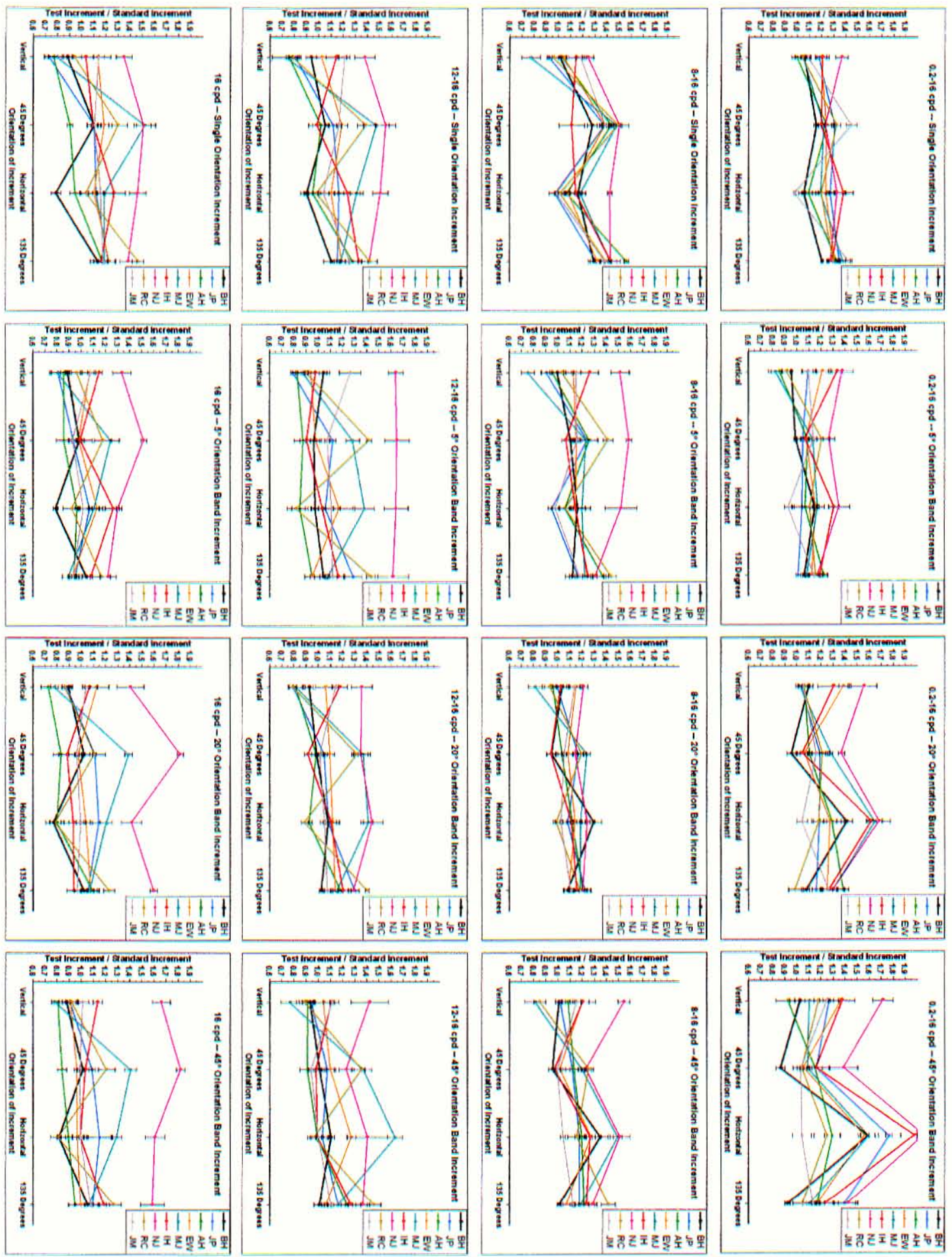

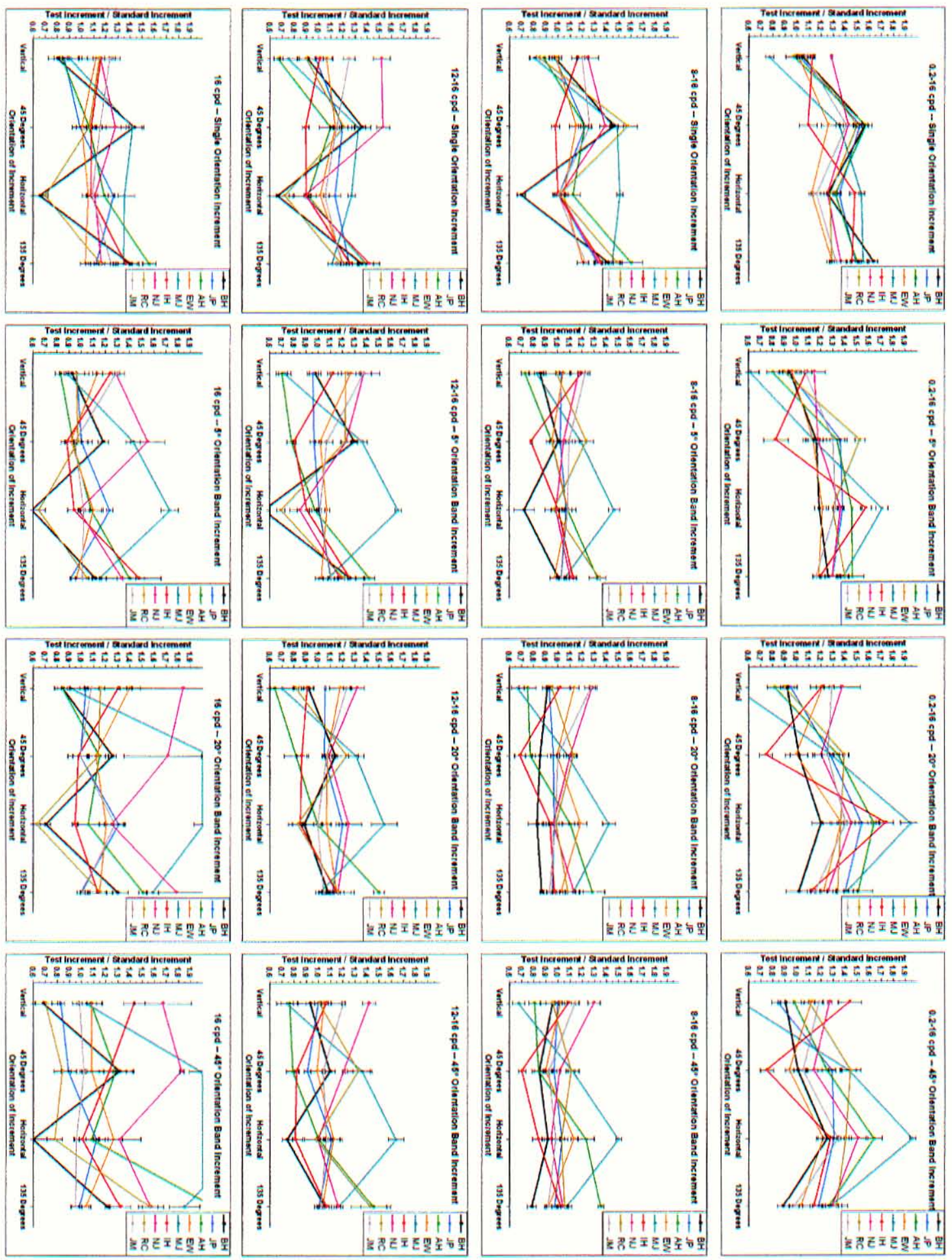


\title{
CURRICULUM VITAE
}

\author{
Bruce C. Hansen \\ University of Louisville \\ Department of Psychological and Brain Sciences \\ 317 Life Sciences Building \\ Louisville, KY 40292 \\ Lab: (502) 852-7710 \\ e-mail: bchans01@louisville.edu \\ www.louisville.edu/ bchans01
}

\section{Education}

University of Louisville

Ph.D. (expected Dec. 2004)

Department of Psychological and Brain Sciences

Major Area: Experimental Psychology

Program: Perception and Neural Sciences

Sub-Program: Vision Science

University of Louisville

M.A.

2003

Department of Psychological and Brain Sciences

Major Area: Experimental Psychology

Program: Vision Science

University of Michigan-Flint

B.S. (with honors) 2000

Department of Psychology

Major Area: Psychology

\section{Publications}

Hansen, B.C. \& Essock, E.A. The relationship between human perceptual performance and the physical attributes of night vision imagery. To appear in Progress in Experimental Psychology Research, Nova Science Publishers, New York (2005).

Zheng, Y., Hansen, B.C., Haun, A.M., \& Essock, E.A. (2005). Coloring night-vision imagery with statistical properties of natural colors by using image segmentation and histogram matching. To appear in Proceedings of IS\&T/SPIE's 17th Annual Symposium on Electronic Imaging 2005, San Jose, CA.

Essock, E.A., Sinai, M.J., DeFord, J.K., Hansen, B.C., \& Srinivasan, N. (2004). Human perceptual performance with non-literal imagery: Region recognition and texture-based segmentation. Journal of Experimental Psychology: Applied, 10, 97-110. 
Gunvant, P.G., Baskaran, M., Vijaya, L., Hansen, B.C., Joseph, I.S., Watkins, R.J., Broadway, D.C., \& O'Leary, D.J. (2004). Comparison of pulsatile ocular blood flow in indians and europeans. Eye (In Press).

Hansen, B.C. \& Essock, E.A. (2004). A horizontal bias in human visual processing of orientation and its correspondence to the structural components of natural scenes. Journal of Vision (Accepted).

Hansen, B.C. \& Essock, E.A. (2004). Influence of scale and orientation on the visual perception of natural scenes. Visual Cognition (In Press).

Zheng, Y., Essock, E.A., \& Hansen, B.C. (2004). An advanced image fusion algorithm based on wavelet transform - Incorporation with PCA and morphological processing. Proceedings of IS\&T/SPIE's 16th Annual Symposium on Electronic Imaging 2004, San Jose, CA.

Zheng, Y., Essock, E.A., \& Hansen, B.C. (2004). An advanced DWT fusion algorithm and its optimization by using the metric of image quality index, Optical Engineering (In Press).

Zheng, Y., Essock, E.A., Hansen, B.C., \& Haun, A.M. (2004). A new metric based on extended spatial frequency and its application to DWT based fusion algorithms. Information Fusion (Under Revision).

Essock, E.A., DeFord, J.K., Hansen, B.C., \& Sinai, M.J. (2003). Oblique stimuli are seen best (not worst!) in naturalistic broad-band stimuli: A horizontal effect. Vision Research, 43, 1329 1335.

Hansen, B.C., Essock, E.A., Zheng, Y., \& DeFord, J.K. (2003). Perceptual anisotropies in visual processing and their relation to natural image statistics. Network: Computation in Neural Systems, 14, 501-526.

\section{Published Abstracts and Presentations}

Essock, E.A., Hansen, B.C., \& Haun, A.M. (2004, May). Assessment of human visual sensitivity to broadband orientations following adaptation to naturalistic orientation biases. Poster session given at the $10^{\text {th }}$ Annual EPSCOR Conference: Pursuing Research Centers of National Stature, Lexington, KY.

Essock, E.A., Hansen, B.C., Zheng, Y., Haun, A.M., \& Gunvant, P. (2004). "Mach bands" in the orientation dimension: An illusion due to inhibition of nearby orientations, [Abstract] to appear in Journal of Vision.

Hansen, B.C., Essock, E.A., \& Haun, A.M. (2004). Visual adaptation and its relation to the "horizontal effect": Implications for visual processing of broadband orientation content [Abstract] to appear in Journal of Vision.

Hansen, B.C., Essock, E.A., \& Haun, A.M. (2004, May). Perceiving broadband oriented content in veridical and altered coordinate systems. Oral presentation given at the $10^{\text {th }}$ Annual EPSCOR Conference: Pursuing Research Centers of National Stature, Lexington, KY. 
Essock, E.A. \& Hansen, B.C. (2003). Seeing the content before the horizon: Visual processing of orientation in natural scenes [Abstract]. Journal of Vision, 3(9), 517a.

Essock, E.A., Hansen, B.C., DeFord, J.K., \& Sinai, M.J. (2003, May). Human visual processing of orientation in real-world imagery. Poster session given at the $9^{\text {th }}$ Annual EPSCOR Conference: Advances in Research Through EPSCOR, Lexington, KY.

Hansen, B.C. \& Essock, E.A. (2003). Human visual processing of orientation and the slope of the amplitude spectra of natural stimuli [Abstract]. Journal of Vision 3(9), 516a.

Hansen, B.C., DeFord, J.K., \& Essock, E.A. (2003). When do humans show a horizontal effect instead of an oblique effect?: An analysis of visual anisotropy across breadth of orientation and spatial frequency broadband content. [on CD-ROM]. Association for Research in Vision and Ophthalmology. Abstract 4091.

Hansen, B.C., Essock, E.A., Sinai, M.J., DeFord, J.K., and Zheng, Y. (2003, May). A frequency-based fusion algorithm to improve human performance with non-literal imagery: Optimization of natural scene statistical properties. Oral presentation given at the $9^{\text {th }}$ Annual EPSCOR Conference: Advances in Research Through EPSCOR, Lexington, KY.

DeFord, J.K., Hansen, B.C., Sinai, M.J., \& Essock, E.A. (2002). Perceptual anisotropy in the salience of broadband image structure. [on CD-ROM]. Association for Research in Vision and Ophthalmology. Abstract 4721.

Hansen, B.C., DeFord, J.K., Sinai, M.J., \& Essock, E.A. (2002). Anisotropic processing of natural scenes depends on scene content [Abstract]. Journal of Vision, 2(7), 500a.

Hansen, B.C., DeFord, J.K., Sinai, M.J., and Essock, E.A. (2002, July). Manipulation of the amplitude and phase spectra of broadband stimuli: Implications for human visual processing of natural scenes. Poster session given at the Sensory Coding and the Natural Environment: Probabilistic Models of Perception Biannual Meeting; Gordon Research Conferences, South Hadley, MA.

Wrobel, T.A., Cheatom, H., Smith, J., Smith, S., \& Hansen, B.C. (2002, August). Accuracy of memory assessment measures in undergraduate and elderly samples. Poster session given at the American Psychological Association annual convention, Chicago, IL.

DeFord, J.K., Hansen, B.C., Sinai, M.J., \& Essock, E.A. (2001). The "horizontal effect": An anisotropy in processing natural scenes. Investigative Ophthalmology and Visual Science, (Supplement), 42 (4), 3310.

Hansen, B.C., DeFord, J.K., Sinai, M.J., \& Essock, E.A. (2001, October). A Perceptual Anisotropy In Processing Natural Scenes. Poster session given at Research Louisville, Louisville, KY.

Wrobel, T.A. \& Hansen, B.C. (2001, August). Thresholds for gender effects in recognition of distorted pictures. Poster session given at the American Psychological Association annual convention, San Francisco, CA. 
Allen, G., Combs, K., Galbreath, S., \& Hansen, B.C. (2000, May). Recognition thresholds for distorted pictures in an elderly sample. Oral presentation given at Meeting of Minds VIII, Dearborn, MI.

Cheatom, H., Dickey, T., \& Hansen, B.C. (2000, May). Recognition threshold for distorted pictures in an undergraduate sample. Poster session given at Meeting of Minds VIII, Dearborn, MI.

Hansen, B.C. (2000, May). Thresholds in distorted object recognition. Poster session given at the Midwestern Psychological Association, Chicago, IL.

Hansen, B.C. (2000, May). Thresholds in distorted picture recognition for normal and early alzheimer participants. Poster session given at Meeting of Minds VIII, Dearborn, MI.

Hansen, B.C. \& Ivey, J. (2000, May). The correlation between depression and memory in elderly compared to student sample. Poster session given at Meeting of Minds VIII, Dearborn, MI.

\section{Relevant Experience}

$2001-2004$

Fall 2004

Fall 2004

Summer-III 2004

June 2004

$2000-2001$

\section{Awards and Honors}

8/03 - 7/04 Grawemeyer Fellow, University of Louisville, Department of Psychological and Brain Sciences.

6/03 - 6/04 Graduate Research Fellow, NASA Kentucky Space Grant Consortium, Bowling Green, KY.
Graduate Research Assistant

Department of Psychological and Brain Sciences

University of Louisville

Course Instructor (this course was team-taught)

Psychology 331: Sensation and Perception

University of Louisville

Graduate Teaching Assistant

Psychology 301: Quantitative Methods in Psychology

University of Louisville

Course Instructor

Psychology 331: Sensation and Perception

University of Louisville

Graduate Research Assistant

Fudan University \& Institute of Neuroscience

Shanghai, China

Lab Instructor

Psychology 201: Introduction to Psychology

University of Louisville 
6/02 - 6/03 Grawemeyer Research Award, University of Louisville, Department of Psychological and Brain Sciences.

6/02 - 6/03 Graduate Research Fellow, NASA Kentucky Space Grant Consortium, Bowling Green, KY.

8/01 - 6/02 Grawemeyer Fellow, University of Louisville, Department of Psychological and Brain Sciences.

2000 Alfred C. Raphelson Prize, Recognition of Outstanding Research Award, University of Michigan-Flint, Department of Psychology. National Collegiate Natural Science Award. All-American Scholar Award. National Collegiate Natural Science Award.

\section{Academic and Professional Societies}

Association for Research in Vision and Ophthalmology

American Psychological Society, Graduate Student Affiliate

Psi Chi National Honor Society in Psychology

Vision Sciences Society

\section{Services and Committees}

2002 - present

Fall 2004

$2002-2003$
Webmaster, Perception and Neural Sciences Program, University of Louisville Alternate Course Director (Psychology 331); served from Aug. $19^{\text {th }}$ to Sept. $10^{\text {th }}$. Graduate Student Orientation Committee

\section{Technical Skills}

Programming Languages:

Operating Systems:

Software Platforms:

Imaging/sensor Systems:
C, C ++ , MATLAB, openGL

Windows NT/XP/2000/ME/98/95

All components of MS Office XP/97, SPSS, NCSS-PASS, Psignifit, Adobe PhotoShop, Illustrator, ImageStyler, \& Premiere, Talon Image Pro, AXUM, DreamWeaver MX, nVidia Talon/IRvista Near-infrared, Infrared, and Image Intensifier sensor systems, Optical Imaging of Intrinsic Signals, STRATUSOCT (direct cross-sectional imaging), IL1700 Research Radiometer 